


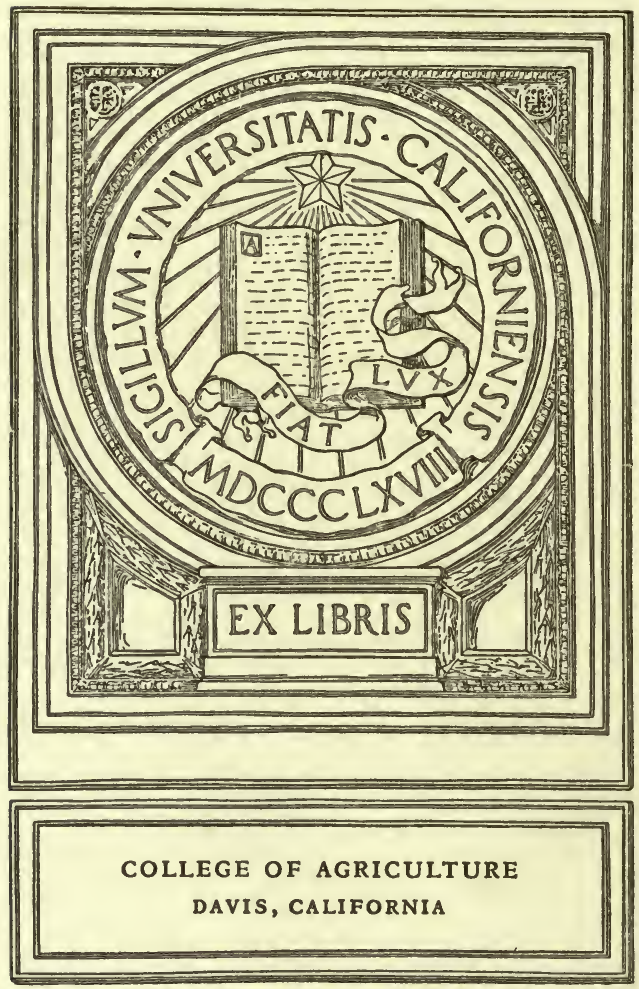


JFE Vaw Nownm 
Digitized by the Internet Archive in 2007 with funding from

Microsoft Corporation 


\section{METHODS USED IN THE EXAMINATION OF MILK AND DAIRY PRODUCTS}




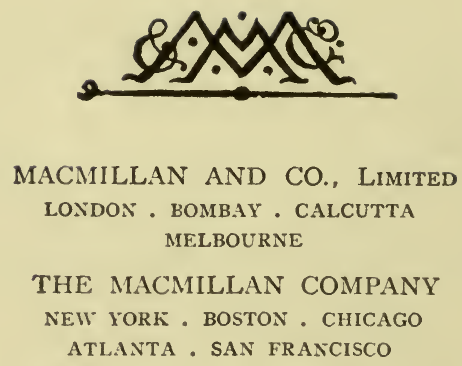

THE MACMILLAN CO. OF CANADA, LTD. TORONTO 


\section{METHODS USED IN THE EXAMINATION OF MILK AND DAIRY PRODUCTS}

$B V^{*}$

DR. CHR. BARTHEL, Experimentalfältet, Stockholm

TRANSLATION BY

W. GOODWIN, M.Sc., PH.D.,

South-Eastern Agricultural College, Wye, Kent

MACMILLAN AND CO., LIMITED ST. MARTIN'S STREET, LONDON

I 910

UNIVERSITY OF CALIFORNIA

LIBRARY

COLLEGE OF AGRICULTURE 
Richari: Clay and Sons, Limited, BREA' STREET HILL, E.C., AND BUNGAYY, SUFFOLK. 


\section{PREFACE}

THE English translation of Dr. Barthel's well-known book is practically a new edition, for the subject matter has been completely revised and considerable additions have been made to it. Those engaged in the examination of milk or dairy products will find in this volume a detailed description of the most reliable methods used in this important branch of analytical work. As many of the methods have been carefully tested by the author himself they are to be regarded as the most satisfactory ones to be followed. The proofs of this edition have been seen and approved by Dr. Barthel.

W. GOODWIN.

Wre, March, 1910. 



\section{CONTENTS}

PART I.-NILK.

INTRODUCTORY . . . . . . . . . . . . . . PAGK 1

The Examisation of Milk . . . . . . . . . . . . 17

A. Sampling . . . . . . . . . . . . . . 17

B. Physical Examination ... . . . . . . . . . . 22

I. Determination of the Specific Gravity of Milk . . . 23

II. Estimation of the Dirt. . . . . . . . . . . 26

C. Chemical Examination.............. . . 30

I. Determination of the Acidity of Milk . . . . . . 30

1. The Reaction of the Milk to Litmus Paper . . . . . 30

2. The Boiling Test . . . . . . . . . . 30

3. The Alcohol Test . . . . . . . . . . . 30

4. Soxhlet-Henkel Method of Titration ....... 31

5. Thörner's Method of Titration . . . . . . . . . 32

6. Dornic's Method of Titration . . . . . . . . . 32

II. Determination of the Dry Matter, or Total Solids. . . 33

III. Determination of the Fat . . . . . . . . . . . 35

A. Scientific Methods . . . . . . . . . 36

1. Soxhlet's Arzometer Method . . . . . . . . 36

2. Wollny's Refractometer Method . . . . . . . 40

3. Extraction Methorls . . . . . . . . . . 47. 47

(a) Adam's Paper Coil Method . . . . . 48

(b) Nilson's Kaolin Methorl . . . . . . . . 50

4. Röse-Gottlieb's Method . . . . . . . . 52

5. Liebermann-Székely's Method . . . . . . 5 5

6. Weibull's Desiccation Method for the Estimation of Fat in Cream . . . . . . . . . 56

B. Practical Methods . . . . . . . . . 58

1. The "Lactocrite" Method . . . . . . . . 58

2. Babcock's Method . . . . . . . . . . 61

3. Lindström's Butyrometer Method . . . . . 65

4. Gerber's Method . . . . . . . . . . 69

5. Sichler's "Sinacid" Butyrometer Method . . . . 77

6. Gerber's "Sal" Methorl ........... 80 
IV. Determination of the Nitrogenous Substances . . . . . ${ }^{\text {PAl.E }} 80$

1. Estimation of the Total Protein . . . . . . . 80

(a) Determination of the Total Nitrogen . . . 80

(b) Ritthausen's Method for Estimating the Total Protein .............. . . 83

(c) Sebelien's Method for Estimating the Total. Protein .............. . . 84

2. Estimation of Cascin . . . . . . . . . 84

3. Estimation of Albumin ... . . . . . . 85

V. Estimation of the Milk Sugar . . . . . . . . . . 85

1. Gravimetric Methods . . . . . . . . . 8.

(a) Alihn-Soxhlet Methorl . . . . . . . . . 8.)

(b) Cupric-Oxide Methorl . . . . . . . . . . . 87

2. Polarimetric Method . . . . . . . . . . . 87

3. The Refractometer Methol . . . . . . . . . 89

VI. Determination of the Ash . . . . . . . . . . . 99

VII. Estimation of the Remaining Constitnents of Milk . . 91

1. Estimation of Citric Acicl . . . . . . . . 91

2. Estimation of Lecithin . . . . . . . . . . . 94

VIII. To Distinguish Raw and Heated Milk . . . . . . . . . 96

(a) Arnold's Guaiacum T'est . . . . . . . . . . . 97

(b) Storch's Paraphenylenediamine Test . . . . . 98

1). Proofs of Milk Adulteration. . . . . . . . . . 100

I. The Adrliticn of Water ........... . 101

1. The Nitrate Test-

(a) Soxhlet's Nitrate Test .......... 101

(b) Fritzmann's Nitrate T'est . . . . . . . . . 102

2. 1)etermination of the Specific Gravity ; the Fat and the Total Solids . . . . . . . . . . 103

3. Determination of the Specific Gravity of Milk Serum 105

4. Determination of the Freezing Point of Milk . . . 106

5. Determination of the Refractive Index of Milk Serum 108

II. The Remoral of Cream ............. . 111

III. Simultaneous Removal of Cream and Adrlition of Water or Separated Milk . . . . . . . . . . 112

The Stall Test.......... . . . . . . 112

1. Calculation of the Added Water . . . . . 113

2. Calculation of the Amount of Cream Abstracted. 114

IV. Admixture with Goats' Milk . . . . . . . . . . 115.

V. The Detection of Artificial Colouring . . . . . . . 116

VI. Addition of Saccharate of Lime . . . . . . . . . 117

The Detection of Saccharose in Milk to which Saccharate of Lime has been adcled . . . . . . . . . 117

The Detection of Lime in Milk . . . . . . . . . 118 
VII The Addition of Preservatives -

1. Carbonate or Bicarbonate of Sorla . . . . . . . . . 119

2. Boracic Acid and Borax . . . . . . . . . 120

3. Salicylic Acid . . . . . . . . . . . . 120

4. Benzoic Acid . . . . . . . . . . . . 121

5. Formalilehyile .............. . . 121

6. Hydrogen Peroxide ............ . . 122

(a) Qualitative Estimation . . . . . . . . 122

(b) Quantitative Estination . . . . . . . 123

The Fermentation Test with Jilk . . . . . . . . 124

The Reductase Test . . . . . . . . . . . 127

PART II. - BUTTER.

PAGE

Eximination of Butter . . . . . . . . . . . . . . . 134

A. Sampling . . . . . . . . . . . . . . . 134

B. The Chemical Analysis . . . . . . . . . . . . 135

I. Determination of the Water ........... 135

II. Determination of the Fat . . . . . . . . . . 137

(a) Scientific Methods . . . . . . . . . 137

1. The Extraction Methor . . . . . . . . 137

2. Riise-Gottlieb Method . . . . . . . . 138

3. Indirect Determination of Fat . . . . . . . 139

(b) Practical Methorls ............. . . 139

Bernstein's Method . . . . . . . . . 139

III. Determination of the Anhyilrous Solids, not Fat . . . 140

IV. Determination of Protein . . . . . . . . . . 141

V. Estimation of Common Salt . . . . . . . . . 141

VI. Proof of Pasteurisation . . . . . . . . . $1+1$

Examitation of the Butter Fat ............. 142

A. Physical Tests . . . . . . . . . . . . 142

I. Determination of the Specific Gravity of Butter Fat (at $100^{\circ}$ C) ................... 142

II. Determination of the Melting Point and the Point of Solidification ............ . . 142

(a) The Melting Point ............ . 142

(b) The Point of Solidification . . . . . . . . 143

III. Determination of the Critical Temperature of Solution in Alcohol ................. . 144 
IV. Determination of the Refractive Index ........ ${ }^{\text {PAGE }}$

B. Chemical Tests . . . . . . . . . . . . . 148

I. The Reichert-Neissl Number . . . . . . . . 148

II. The Koettstorfer Number . . . . . . . . . . . 152

III. The Hehner Number . . . . . . . . . . . . 153

IT. The Iodine Number . . . . . . . . . . . 153

(a) Hiibl's Methorl . . . . . . . . . . . . . 154

(b) Wiss' Methor ............... 155

V. Determination of the Amount of Free Acid . . . . 156

C. Detection of the Adulteration in Butter . . . . . . . . 157

I. Admixture of Foreign Fats ... . . . . . . 157

(a) Admixture with Margarine . . . . . . . . 158

1. Determination of the Refractive Index of the Fat 159

2. The Crismer Number .......... . 161

3. The Reichert-Meissl Number. . . . . . . . 161

4. The Koettstorfer Number . . . . . . . 162

5. The Phytosterine Acetate Test . . . . . . 162

6. The Latent Colouring of Margarine . . . . . 166

(h) Admixture with Cocoa-nut Oil . . . . . . . 168

1. Polenske's New Butter Number . . . . . . 169

Qualitative Tests for Cocoa-nut Fat in Butter. 173

II. Mixing of Water with Butter. . . . . . . . . . 175

III. Addition of Preservatives . . . . . . . . . 176

D. Detection of Colouring Matter in Butter. . . . . . . . 176

\section{PART III.-CHEESE.}

INTRODUCTORY . . . . . . . . . . . . . . . . . . ${ }^{{ }^{P A}} 180$

Analysis of Chiese . . . . . . . . . . . . . . . . . . . 184

A. Sampling . . . . . . . . . . . . . . . . 184

I. Determination of the Percentage of Water . . . . . 18.5

II. Determination of Fat . . . . . . . . . . 186

(a) Scientific Methods . . . . . . . . . 186

1. The Extraction Method ......... 186

2. The Schmid-Bondzynski Method . . . . . 187

3. The Röse-Gottlieb Method . . . . . . . . . 187

(b) Practical Methods . . . . . . . . . . 189

Gerber's Test . . . . . . . . . . 189

III. Determination of the Nitrogenous Substances . . . . . . 190

(a) Fstimation of the Total Nitrogen . . . . . . 190

(b) Estimation of the Water-Soluble Nitrogenous Compounds .............. . . . 190

IV. Determination of the Ash ........... 190

B. Detection of Adulteration . . . . . . . . . . . . . . 191

C. Testing of Rennet. . . . . . . . . . . . . . . . . 191 
PART IV.-PRESERVED MILK AND OTHER MILK PREPARATIONS.

Examivatios of Preserved Mith and Other Milk Preparatioss Pagk

I. Sterilised and Pasteurised Milk . . . . . . . . 210

II. Buddised Milk . . . . . . . . . . . . . . . 212

III. Condensed Milk . . . . . . . . . . . . . . . . . . 213

IV. Desiccated Mlilk or Milk Powder............ 214

V. Casein ................. . . 215

PART V.-DECOMPOSITION PRODUCTS OF MILK, BUTTER, AND CHEESE.

2. Volatile Fatty Acids . . . . . . . . . . . . . . . 219

(a) Qualitative Fstimation ............ . 220

(b) Quantitative Estimation ............. 221

3. Alcohol ... . . . . . . . . . . . . 226

II. Decomposition Products of Butter . . . . . . . . . . 229

1. Determination of the Degree of Rancidity . . . . . . . . 230

2. Determination of the Degree of Oxidation . . . . . . . 232

III. Decomposition Prolucts of Cheese . . . . . . . . . . . . 233

INTRODUCTORY . . . . . . . . . . . . . . . . . . 233

1. Determination of the Degree of Acidity . . . . . . . 233

2. Volatile Fatty Acids . . . . . . . . . . . . . 233

3. Acid Number of Cheese Fat . . . . . . . . . . . 234

4. Nitrogenous Substances . . . . . . . . . . . $23 \tilde{5}$

APPENDIX-TABLE . . . . . . . . . . . . . . . 236 



\title{
METHODS USED IN \\ THE EXAMINATION OF MILK AND \\ DAIRY PRODUCTS
}

\author{
PART I-MILK
}

\section{INTRODUCTORY}

MrLK is, as is well known, the secretion of the mammary glands of mammalia, and in this volume, wherever the word milk is employed alone, it will be understood to apply to the milk of the cow, for this is much more important, for domestic purposes, than the milk of any other animal.

Milk which is secreted by a healthy udder is an opaque, white emulsion with a porcelain-like appearance. Sometimes it has a tinge of yellow or blue, according to the amount of fat and the deeper or lighter colour of the fat. Milk is transparent only when in very thin layers. The taste of milk is satisfying, pleasant, and somewhat sweet, and the smell is reminiscent of the animal, but not unpleasantly so.

Milk is heavier than water, the specific gravity lying between 1.029 and 1.033 , according to Fleischmann, ${ }^{1}$ when the sample is taken from five or more cows, or when it is from two or three milkings. The milk of individual cows or that from a single milking has a specific gravity between 1.028 and 1.034. It may be taken that the average specific gravity is 1.0315 when determined, as in the above cases, at a temperature of $15^{\circ} \mathrm{C}$.

The viscosity of milk compared with water is $220: 100$ at $0^{\circ}$, whilst at $30^{\circ}$ it is $170: 100$. There is a considerable increase of viscosity at the lower temperatures, and on that account the foam which forms on the surface of cold milk persists for a long time.

1 Lehrbuch der Milchwirtschaft. Fourth Edition. Leipsic, 1907. 
The freezing point of milk lies between $-0.55^{\circ}$ and $-0.57^{\circ} \mathrm{C}$, that is, below that of water. According to J. Winter and E. Parmentier, ${ }^{1}$ the freezing-point of milk is almost constant, $-0.555^{\circ} \mathrm{C}$.; the author ${ }^{2}$ also has found that with mixed milk this figure is practically correct. Milk differs from water in having its greatest density at freezing point, whereas that of the latter is, as is well known, at $4^{\circ} \mathrm{C}$.

The boiling point of milk is about $0 \cdot 2^{\circ}$ higher than that of water when both are under the same atmospheric pressure.

The specific heat of milk varies according to the amount of dry matter it contains, and Fleischmann ${ }^{3}$ gives the average as 0.9457 compared with water as unity. According to later and more exact investigations by C. Schnorf, ${ }^{4}$ the specific heat of milk is higher than this, and on an average reaches 1.042.

The refractive index of milk, according to the most recent observations, is between 13430 and 1.3445 at $15^{\circ} \mathrm{C}$. The resistance which milk offers to the passage of an electric current has been found to vary considerably by different observers, but F. Petersen, ${ }^{5}$ to whom the latest experiments on this subject are due, gives it as varying between 186 and 304 ohms at $15^{\circ} \mathrm{C}$., with an average of 231.64 ohms. Where the experiments were made with mixed milk the resistance at $15^{\circ} \mathrm{C}$, varied between 204 and $255 \mathrm{ohms}$.

Milk has an amphoteric reaction, it being both alkaline and acid to litmus paper. This is because milk contains phosphates which have an alkaline reaction, and others that have an acid reaction.

To phenolphthalein, however, milk is acid, and requires a not inconsiderable amount of alkali in order to bring the liquid to a neutral reaction with this indicator. The quantity of alkali required depends upon the degree of acidity of the milk. If $N / 4$ alkali is taken for the titration, as recommended by Soxhlet-Henkel, it is found that on an average 7 c.c. are required to neutralise 100 c.c. milk. On the other hand, to make 100 c.c. milk neutral to lacmoid requires, according to Courant, ${ }^{6} 41$ c.c. $N / 10 \mathrm{H}_{2} \mathrm{SO}_{4}$.

1 Revue Générale du Lait, Vol. III, p. 193.

3 Journal für Landwirtschaft, 1902, Vol. L, 33-76.

4 Revue Générale du Lait, Vol. IV, p. 313.

5 Untersichungen über den elektrisch. Widlerstand der Milch. Inaug. Diss. Kiel, 1904. 
When milk is heated, the only apparent change is the formation of a skin on the surface when the temperature rises above $50^{\circ} \mathrm{C}$. This skin consists of casein, which, in consequence of the rapid evaporation, has lost a portion of the water which it contains; on stirring the milk the skin again dissolves.

Milk is naturally intended for the nourishment of newly-born animals, but from the earliest times man has understood by various means how to prolong the period of milk production with some domestic animals such as the cow, goat, and sheep. Thus it is that now the production of milk is not limited to the time of suckling the young, but continues much longer, often, in fact, until shortly before the birth of the next ealf, kid, \&c.

If milk is to serve exclusively for nourishing the young, it must, of course, contain all the nutrients required by the animal organism for its maintenance and growth, and these are protein, fat, carbohydrates, and inorganic salts. In addition, milk also contains small quantities of various substances which, although they possess no direct feeding value, have nevertheless an important physiological effect. The milk of all species of animals contains the whole of the constituents mentioned above, but the quantities in which they are present vary in different cases.

Cow's milk contains almost nine-tenths of its weight of water; the other components, which may be got by evaporating off the water and are known together as "dry matter" or "total solids," represent slightly more than one-tenth the weight of normal milk. According to Fleischmann, the average composition of milk, based upon a large number of investigations, is :-

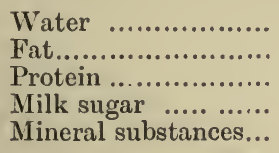

\begin{tabular}{|c|c|c|c|}
\hline $\begin{array}{c}\text { Average } \\
87.75\end{array}$ & $\begin{array}{l}\text { umbers. } \\
\text { cent. }\end{array}$ & $\begin{array}{l}\text { Limits of } \\
86.5-89.5\end{array}$ & $\begin{array}{l}\text { iation. } \\
\text { cent. }\end{array}$ \\
\hline $3 \cdot 40$ & ," & $2 \cdot 7-4 \cdot 3$ & \\
\hline $3 \cdot 50$ & ", & $3 \cdot 0-4 \cdot 0$ & ," \\
\hline $4 \cdot 60$ & ", & $3 \cdot 6-5 \cdot 5$ & ," \\
\hline $0 \cdot 75$ & ", & $0.6-0.9$ & ", \\
\hline & ient & & ent. \\
\hline
\end{tabular}

The above figures apply to mixed milk from a large number of cows; with single animals the limits of variation are often considerably greater.

I Lehrbuch der Milchwirtschaft. Fourth Edition. Leipsic, 1907. 
The average amount of dry matter is given by Fleischmann as 12.25 per cent., this figure applying to the mixed milk from a large number of herds in Germany. The percentage of fat in the dry matter is usually 27.75 per cent., the specific gravity being 1.334 at $15^{\circ} \mathrm{C}$. When the dry matter is freed from fat it is on an average 8.85 per cent. of the weight of the milk, and the specific gravity lies about $1 \cdot 6$.

Fat is present in milk in the form of an emulsion, that is, in an immense number of minute globules which have a diameter varying between 0.0016 and $0.01 \mathrm{~mm}$. (see Fig. 1). In some

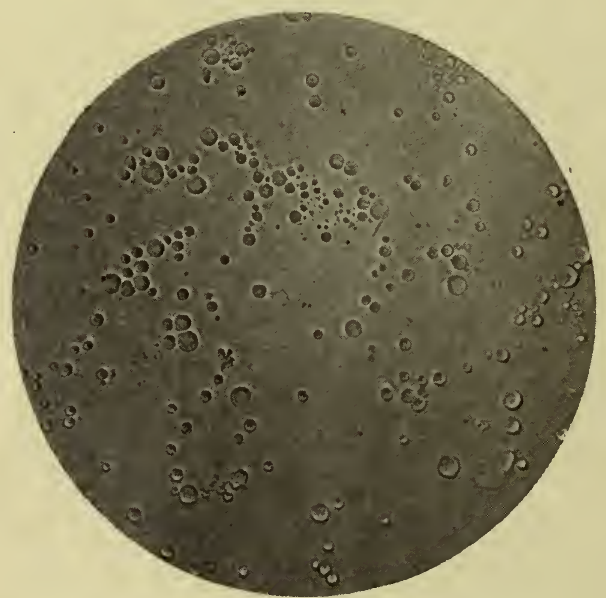

Fic. 1.-1 :400. Barthel photo. (Microphotograph of the Fat Giobules in Milk.)

cases, though, the fat globules vary as much as $0.0001-0.022$ $\mathrm{mm}$., but the average diameter may be taken as approximately $0.003 \mathrm{~mm}$. It is these microscopically small globules of fat which along with the colloidial casein give to the milk its peculiar white, opaque appearance. With regard to the number of fat globules usually present in milk, the author has calculated that the average quantity per cubic millimetre of milk is 3,000,000 where a large number of animals are concerned. These figures agree closely with those given by Babcock, ${ }^{1}$ Woll, ${ }^{2}$ and Gutzeit. ${ }^{3}$ The number and size of the

1 Fourth Ann. Report N.Y. Agr. Expt. Sta., Geneva, 1886, p. 226.

2 Agricultural Science, Vol. VI, No. 10, p. 444.

${ }^{3}$ Landw. Jahrbücher, Vol. XXIV, 1895, p. 539. 
fat globules vary, however, very considerably in the different breerls, and also in individuals at different stages of the period of lactation. In the same volume of milk towards the end of the period of lactation, the number of fat globules is greater than at the beginning of the period.

The debated question as to whether the fat globules are surrounded by a membrane of any sort is not definitely decided, but the present views are that none exists, and that the surface tension keeps the globules separate from one another, as is seen in other emulsions.

Milk fat is fluid at ordinary temperatures and is in an undercooled state. It is only in the process of butter-making that the fat passes into the solid form. As the specific gravity of milk fat is 0.93 at $15^{\circ} \mathrm{C}$, the globules are lighter than the liquid in which they are present, and so they tend to rise to the surface. As is well known, milk when left to stand gradually gains a layer of these fat globules on the upper surface, giving what is known in daily life as cream.

The fat of cow's milk is, at ordinary temperatures, a soft yellowish mass with a pleasant taste and only a faint smell. It melts between $31^{\circ}$ and $36^{\circ} \mathrm{C}$., and the point of solidification is from $24^{\circ}-19^{\circ} \mathrm{C}$. According to Gutzeit, ${ }^{1}$ the refiactive index of butter fat is generally 1.462 at $40^{\circ} \mathrm{C}$.

The chemical composition of milk fat shows it to be composed of a mixture of the triglycerides of volatile and non-volatile fatty acids. It is characteristic of other animal fats that they only contain a small number of the different glycerides, whereas in milk fat there are not less than ten and it is possible that as many as twelve different glycerides are present.

Of these there are the glycerides of four volatile fatty acids, viz., butyrin, capronin, caprylin, and caprin, and the glycerides of six non-volatile fatty acids, viz., myristin, palmitin, stearin, arachin, laurin, and olein. The constant presence of formin and acetin has not yet been satisfactorily proved. It is probable that there is no difference in the composition of the small and large fat globules.

The quantity of fat found in milk is very variable; none of the other constituents of the milk is subject to such large fluctuations as is the fat. In the case of individual cows the 
amount of fat in separate milkings may vary from 1 to 8 per cent. When, however, the mixed milk from several animals is examined, it is found that the fluctuations are much smaller, being then from 2.5 to 4.5 per cent., with an average, for Germany, of 3.4 per cent.

The variations in the fat content of milk are partly constant and partly accidental. In the case of a single cow, breed, individuality, and the stage of the period of lactation are the chief factors which influence the amount of milk fat.

As regards the breed, it is found that Jersey cattle give the richest milk, whilst that from Low Country breeds, such as East Friesland, is poorest in fat. The milk of Jersey cows contains on an average 5 per cent. of fat, whilst that of East Friesland or Dutch cows only contains $3 \cdot 1-3 \cdot 2$ per cent. When, however, the quantity of milk is considered, it is seen that the Low Country cattle give an average of 670-780 gals. per annum, whilst the yield of a Jersey cow is only some 510-560 gals. All other breeds have a milk yield somewhere between that of Jersey and Low Country cattle.

The individuality of the animal also plays a very important part, for there are cows which, in all circumstances, give milk with a remarkably high or low percentage of fat, and often this peculiarity is transmitted to their offspring. This fact is of great importance when a good milking herd is being built up.

The amount of fat in the milk is at its lowest after calving, and rises without interruption almost to the next time of calving. Here, too, the quantity of fat stands in inverse proportion to the milk yield, for the latter is largest (as regards one day) immediately after calving, and then gradually decreases until the cow becomes "dry."

The percentage of fat varies very much in the different portions of one milking, for the first are found to contain only small quantities of fat, whereas the succeeding fractions become richer and richer until the last drops are very rich indeed.

Accidental factors which may influence the fat content of the milk are, for instance, sudden changes in the food, unskilful treatment of the cows by their attendant, or anything that disturbs or frightens them.

On the other hand, it is impossible, except for a short period, to raise the amount of fat in the milk by the aid of specific 
foods. It is true that by the use of more nutritious food the yield of milk may be increased, but the proportions in which the components of the milk stand to one another cannot be changed.

The protein substances in the milk are of three kinds: the casein, the lacto-albumin, and the lacto-globulin. Casein is the most important of these, and is present in the largest quantity, there being 85 per cent. of the protein of milk in this form, 15 per cent. as lacto-albumin, and only traces of lacto-globulin. In other words, of the 3.5 per cent. of protein in the milk, 2.9 per cent. are casein, and 0.6 per cent. are albumin and globulin.

Casein is present in milk not in a dissolved, but in a suspended or colloidal condition, which can easily be proved by allowing milk to pass through a filter of unglazed porcelain. ${ }^{1}$ After this operation a white mass is found on the surface of the filter, which consists of almost the whole of the casein of the milk together with the fat which was present. As has already been mentioned, the casein also assists in giving the opaque, porcelainlike appcarance to milk. According to its chemical composition, casein is a nucleo-albumin and is distinguished from other albumins, above all by its phosphorus content and its behaviour towards rennet. Casein has the following chemical composition : ${ }^{2}$

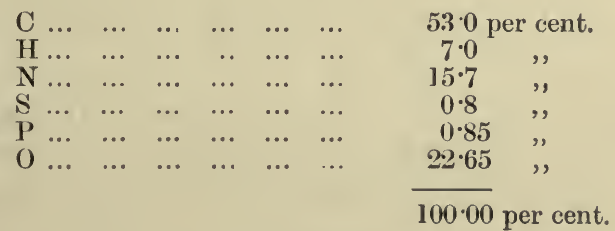

Casein behaves like a fairly strong acid which dissolves in water on the addition of a small quantity of alkali; it also dissolves in water in the presence of calcium carbonate, from which it drives out carbon dioxide. In pure water casein, however, is not soluble.

Probably casein is present in milk in combination with lime as a lime-casein compound. Söldner has prepared two limecasein compounds (di- and tri-calciumcasein) which contain 1.55 and 2.36 per cent. CaO respectively.

1 Duclaux, Le Lait, Études chimiques et microbiologigues, Paris, 1887.

${ }^{2}$ Hamnarsten, Lehrbuch der Physiologischen Chєmie. 
The most characteristic property of casein is that of curdling when acted upon by rennet in the presence of a sufficient quantity of a soluble lime salt. In the absence of lime salts casein is not coagulated by rennet. The casein does, however, undergo a change when acted upon by rennet in the absence of lime, for if lime salts are afterwards added a coagulum is obtained, which possesses exactly the same properties as those of the curd obtained in the normal way. Rennet, therefore, acts upon casein in the absence of lime salts, but the latter are necessary for the precipitation of the curd.

Hammarsten, who was the first to demonstrate these conditions, ${ }^{1}$ is also the original proposer of a theory regarding the coagulation of milk by rennet, which is, briefly, the following: Under the action of the rennet the casein is split up, and, gives on the one hand a body which is only slightly soluble and which stands in very close relation to casein; this he calls paracasein, and it is the main product of the cleavage. On the other hand, there remains in solution a small quantity of an easily soluble albumin-like substance which is poorer in carbon and nitrogen than the paracasein and is named whey-albumin.

Casein can be separated from milk by means of acids or certain solid salts, e.g. common salt. The simplest method for obtaining the casein from milk is to dilute it with four times its volume of water and then add acetic acid up to 1 part per 1000. The precipitated casein is then repeatedly dissolved in water with the help of a little alkali, reprecipitated with acetic acid, and carefully washed. Finally, the casein is freed from traces of fat by treatment with alcohol and ether.

Lacto-albumin was first prepared in a pure state from milk by Sebelien, ${ }^{2}$ who gives its composition as :

$$
\begin{array}{llllll}
\mathrm{C} & \ldots & \ldots & \ldots & \ldots & \ldots \\
\mathrm{H} & \ldots & \ldots & \ldots & \ldots & \ldots \\
\mathrm{N} & . . & \ldots & \ldots & \ldots & \ldots \\
\mathrm{S} & . & \ldots & \ldots & \ldots & \ldots \\
\mathrm{O} & . . & \ldots & \ldots & \ldots & \ldots
\end{array}
$$$$
\begin{gathered}
52 \cdot 19 \text { per cent. } \\
7 \cdot 18 \quad, \\
15 \cdot 77 \quad \text { ", } \\
1.73 \quad \text { ", } \\
23 \cdot 13 \quad \text { ", }
\end{gathered}
$$

$100 \cdot 00$ per cent.

Its properties resemble those of the albumins in general, but it is distinguished from serum-albumin by its considerably

1 Maly's Jahresber. Vols. II and IV ; also Hammarsten, “Zur Kenntnis des Kaseins und der Wirkung des Labferments," Nova Acta Rey. Soc. Scient., Upsal., 1877.

2 Zeitschrift für physioloy. Chem., Vol. IX. 
smaller specific rotation, $a_{[\mathrm{D}]}=-37^{\circ}$. When milk is heated to about $70^{\circ} \mathrm{C}$. the albumin coagulates. Lacto-globulin was also first prepared from milk by Sebelien, ${ }^{1}$ who saturated milk with common salt, which causes the precipitation of the casein, and then added magnesium sulphate to the filtrate until saturated. In its properties lacto-globulin closely resembles serum-globulin, with which it is perhaps identical.

Milk sugar or lactose, $\mathrm{C}_{12} \mathrm{H}_{22} \mathrm{O}_{11}+\mathrm{H}_{2} \mathrm{O}$, is a disaccharide, which on hydrolysis is split up into two hexose sugars, dextrose and galactose. Milk sugar is only found in milk, from which it is easily prepared by evaporating the whey left in cheesemaking, after first coagulating the protein substances by means of heat. After repeated crystallisation, milk sugar is obtained in the form of colourless, rhombic crystals with 1 molecule of water of crystallisation. When heated to $130^{\circ}-140^{\circ} \mathrm{C}$., the water of crystallisation is easily given up, and at $170^{\circ}-180^{\circ} \mathrm{C}$. the milk sugar passes into a brown, amorphous mass (lactocaramel) with the formula $\mathrm{C}_{6} \mathrm{H}_{10} \mathrm{O}_{5}$. Milk sugar dissolves in 6 parts cold and in 2.5 parts boiling water, and the solution is only slightly sweet. In alcohol and ether, milk sugar is insoluble. The aqueous solution of milk sugar is dextrorotary, and the power of rotation which is obtained constant by heating the solution to $100^{\circ} \mathrm{C}$. is $a_{[\mathrm{p}]}=+52.5^{\circ}$.

Ordinary yeast does not ferment milk sugar, but under the influence of certain yeasts, which were discovered by Duclaux, ${ }^{2}$ it can be split up into alcohol and carbon dioxide just as grape sugar is. Such yeasts are of great importance in the preparation of koumiss and kephir, beverages which have long been made by Mongolian races and those living in the Caucasus. Koumiss is prepared from mare's milk, and kephir from cow's milk.

'The effect of the action of these specific milk sugar-fermenting yeasts along with certain bacteria is to give rise to an alcoholic and also an acid fermentation, whereby lactic acid is formed and the cascin undergoes changes which render it more easily assimilable.

Certain micro-organisms, the so-called lactic acid bacteria, cause milk sugar to be changed into lactic acid according to the following equation:

$$
\mathrm{C}_{12} \mathrm{H}_{22} \mathrm{O}_{11}+\mathrm{H}_{2} \mathrm{O}=4 \mathrm{C}_{3} \mathrm{H}_{6} \mathrm{O}_{3} \text {. }
$$

1 Loc. rit.

2 Ann. de l'Inst. Pasteur, Vol. I, 1887. 
The lactic acid formed in this way precipitates the casein of the milk, a process which is so familiar in the ordinary souring of milk.

Milk sugar reduces an alkaline copper solution (Fehling's solution) on warming, and with bases forms saccharates which can be precipitated from their aqueous solutions by alcohol. The mineral salts of milk, which can be obtained by carefully reducing milk to ash, consist of compounds of potassium, sodium, calcium, magnesium, and iron, with phosphoric, sulphuric, and carbonic acids. It is to be noticed, though, that undoubtedly the carbonic acid is formed from the organic constituents of the milk during the combustion, and in the same way the sulphuric acid may be regarded as a decomposition product of the protein of the milk. ${ }^{1}$

Further, in calculating the amount of phosphoric acid, the phosphorus in the casein must not be forgotten, for, as has been seen, this constitutes 0.85 per cent. of the casein.

If the carbon dioxide, the sulphuric acid, and part of the phosphoric acid are deducted, the following average composition for the ash of milk is obtained, the metals being calculated as oxides : ${ }^{2}$

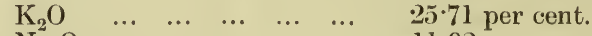

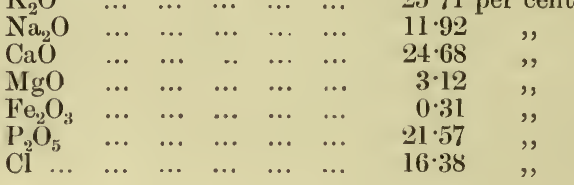

A consideration of the above figures shows at once that the chlorine and the phosphoric acid are not nearly sufficient to combine with all the bases. Even when it is borne in mind that a certain quantity of the lime was in combination with the casein, there is still a considerable excess, for the chlorine and phosphoric acid are, without doubt, combined with the

1 According to Steinegger and Allemann (Landw. Jahrb. d. Schweiz, 1905, Vol. VIII), $\mathrm{H}_{2} \mathrm{SO}_{4}$ is found in milk to the extent of $0.0823-0.1311 \mathrm{~g}$. per litre.

${ }_{2}^{2}$ Fleischmann, Lehrbuch der Milchwirtschaft. Fourth Edition. Leipsic, 1907 ; p. 56. 
alkali metals. There must therefore be another acid present in the milk, and that an organic one. Such an acid has been shown by Henkel ${ }^{1}$ to be a constant constituent of milk, and he proved it to be citric acid. By calculation, it is found that about 0.25 per cent. citric acid is necessary to combine with the residual lime. Recent investigations by Beau ${ }^{2}$ lead to the conclusion that the amount of citric acid in milk is on an average $0 \cdot 2$ per cent.

According to Söldner, ${ }^{3}$ the milk salts would be present in the following proportions in the milk:

\begin{tabular}{|c|c|c|c|c|c|}
\hline Sodium chloride. & $\cdots$ & & & \multicolumn{2}{|c|}{$10 \cdot 62$ per cent. } \\
\hline Potassium chloride & $\ldots$ & $\ldots$ & $\ldots$ & $9 \cdot 16$ & , \\
\hline Monopotassium phosphat & & $\ldots$ & $\ldots$ & $12 \cdot 77$ & ", \\
\hline Dipotassium phosphate & $\ldots$ & ... & $\ldots$ & $9 \cdot 2 \cdot 2$ & , \\
\hline Calcium citrate & .. & $\ldots$ & $\ldots$ & $5 \cdot 47$ & ," \\
\hline Dimagnesium phosphate & $\ldots$. & $\ldots$ & $\ldots$ & $3 \cdot 71$ &, \\
\hline Magnesium citrate $\quad .$. & $\ldots$. & ... & $\ldots$ & $4 \cdot 05$ & ", \\
\hline Dicalcium phosphate... & ... . & $\cdots$ & $\cdots$ & $7 \cdot 42$ & , \\
\hline Tricalcium & $\ldots$. & $\ldots$ & $\ldots$ & $8 \cdot 90$ & ", \\
\hline Calcium citrate $\ldots$... & $\cdots$. & $\cdots$ & $\cdots$ & $23 \cdot 55$ & ", \\
\hline Lime combined & caseir & & $\cdots$ & $5 \cdot 13$ & $"$ \\
\hline
\end{tabular}

The salts are present in the milk in a completely dissolved state, with the exception of a part of the calcium phosphate which is there as a solid in a finely-divided form.

Amongst the other constituents of milk are certain chemical substances which are found there either constantly or accidentally, as well as solid bodies which also may be present regularly or irregularly. Amongst those substances which are constantly found in milk, although only in very small quantities, mention may first be made of lecithin. This substance, in spite of the insignificant amount which is present, is of very great physiological importance.

From the investigations of F. Bordas and Sig. de Raczkowski ${ }^{4}$ it is known that $0.065 \mathrm{~g}$. of lecithin is the average quantity found in 100 c.c. of milk, but this amount varies with the period of lactation, and in such a manner that immediately

1 "Zitronensäure als normales Bestandteil der Milch," Die Landwirtsch. Veršchsstation., 1891, Vol. XXXIX, p. 143.

2 Revue Générale du Lait, Vol. III, 1904, p. 385.

3 "Die Salze der Milch und ihre Beziehungen zu dem Verhalten des Kaseins," Die Landwirtsch. Versuchsstation., 1888, Vol. XXXV, p. 361.

\Compt. rend. de l'Acad. des Sciences, Vol. CXXXIV, p. 1592, and Vol. CXXXV, pp. 302 and 354. 
after calving it is at the maximum (0.091 g. per 100 c.c.), and decreases from that point.

An organic phosphorous compound-nuclein-is also found in milk and comes from the leucocytes, which are always present, and from the nuclei of the epithelial cells of the mammary gland. The following have also been detected with certainty : Urea, about 0.007 per cent., ${ }^{1}$ and cholesterine, ${ }^{2}$ whilst the presence of hypoxanthin, leucine, creatinin, and peptone is very doubtful.

In the last few years a number of different kinds of enzymes have been found to be present in milk.

Babcock and Russell ${ }^{3}$ have shown that milk contains an enzyme which possesses the power of dissolving protein substances in neutral or slightly alkaline solutions. This enzyme, which they have named galactase, resembles trypsin, but is distinguished from it by the power which the former has of giving rise to ammonia when it acts upon milk for a short time. According to Babcock and Russell the galactase plays an important part in the ripening of cheese, owing to the power which it possesses of dissolving protein substances, but other observers, such as Bockhout and Ott de Vries, ${ }^{4}$ do not agree that it has any such action. Galactase is undoubtedly a mixture of enzymes, but the presence in milk of some proteoclastic ferment is at the present time held to be proved.

Cow's milk also contains a diastasic enzyme, a catalase, a peroxydase, and an aldehyde-reductase. ${ }^{5}$ Whether it also contains, as Stoklasa ${ }^{6}$ suggests, an enzyme capable of causing the fermentation of milk sugar has not yet been satisfactorily proved.

Certain gases are also normal components of milk, and Thörner ${ }^{7}$ found that in one litre 57-80 c.c. gas were present. Experiments by Marshall ${ }^{8}$ show that in freshly-drawn milk

1 Fleischmann, Das Molkereiwesen, Braunschweig, 1875, p. 33.

2 Schmidt-Mühlheim, Pfüger's Archiv, Vol. XXX, p. 379:

3 S. M. Babcock and H. L. Russell, "Unorganised Ferments of Milk, a new Factor in the Ripening of Cheese." The Fourteenth Annual Report of the Wisconsin Agricultural Experiment Station, Decenber, 1897.

4 "Untersuchungen über den Reifungsprozess des Edamer Käse," Centralblatt für Bakt. Section 2, Vol. V, p. 304.

${ }_{5}$ H. Smidt, Hygienische Rundschau, 1904, 14, 1137.

6 Archiv für Hygiene, Vol. L, 1904, p. 165.

7. Milch-Zeitung, 1893, p. 58.

8 Centralblatt f. Bakteriologie, Section 2, Vol. IX, p. 313. 
a mixture of gases having the following average composition is found :

$$
\begin{aligned}
& \begin{array}{lllllll}
\mathrm{CO}_{2} & \ldots & \ldots & \ldots & . . & \ldots & 59 \cdot 63 \text { per cent. }
\end{array} \\
& \begin{array}{llllllllll}
0 & \ldots & \ldots & \ldots & \ldots & \ldots & 13 \cdot 17 &
\end{array} \\
& \begin{array}{llllll}
\text { Other gases } & \ldots & \ldots & \ldots & 27 \cdot 20 \quad,
\end{array} \\
& 100 \cdot 00 \text { per cent. }
\end{aligned}
$$

Finally, certain foreign substances can find their way by accident into the milk, as, for example, from the food or from medicine which the animals have taken; these cases, though, are quite exceptional. Of those extraneous bodies which are constantly found in milk the first place is taken by the white blood corpuscles, or leucocytes, which pass directly from the blood into the milk through the thin walls of the alveoli. The presence of leucocytes and their importance will be discussed later in the section dealing with the differences between unheated and heated milk. In addition to the leucocytes, milk also contains other cells and fragments of cells which have arisen from the alveolar epithelium of the mammary gland. Epidermis cells, which have come from the outside of the udder and from the milker's hands, are found almost without exception in milk. Further, in every sample of milk there are found considerable, although varying, numbers of microorganisms; they are chiefly bacteria, but moulds and yeasts are also present. The solid matter which accidentally finds its way into milk is composed of small, light particles, which are blown about in the stall and so fall into the pail during milking. All these substances comprise the dirt of milk, and the amount of this varies according to the degree of cleanliness in the stall, and particularly to the precautions that are observed during milking.

If the milk is mechanically separated by means of centrifugal force, these solid impurities, along with some casein and quantities of white blood corpuscles, cells and cell fragments, collect as a grey sticky mass on the sides of the separator drum. This mass, the so-called separator slime or mud, has an analysis somewhat as in the table on page $14 .^{1}$

If this slime is examined microscopically it is seen that along with the mass of bacteria, white blood corpuscles, and other

1 Chr. Barthel: "Sur la composition de la boue de centrifuge," Revue Gén. du Luit, Vol. I, p. 193. 
cells, there are fragments of dung and fodder, hair from animals and human beings, vegetable fibres, grains of sand, parts of insects-in short, everything that can be found in the dust of the stall. The amount of separator slime can vary from $0.03-0.25$ per cent. of the weight of the milk, but generally it does not exceed $0 \cdot 1$ per cent.

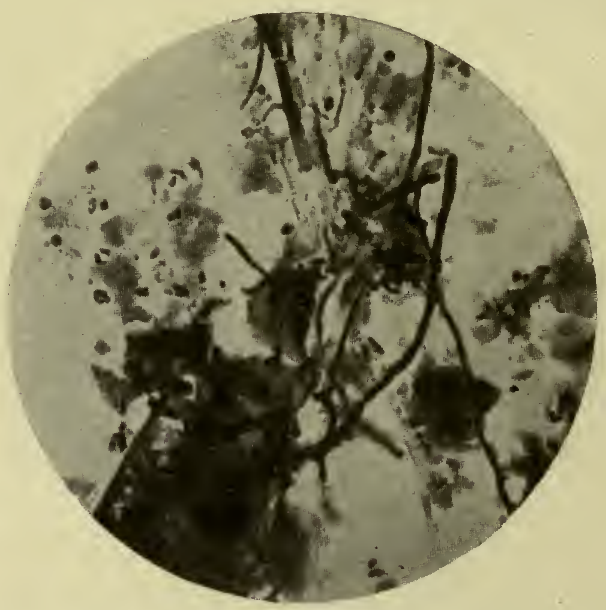

FIG. 2.-1 : 350. Barthel photo. Microphotograph of separator slime. ${ }^{1}$

The above curtailed description of the properties and composition of cow's milk applies only to milk under normal conditions, for immediately after calving there are considerable differences both in its nature and the compounds which it contains. Directly after the birth of the calf the milk, which

\begin{tabular}{|c|c|c|c|}
\hline $\begin{array}{llllll}\text { Water } & \ldots & \ldots & \ldots & \ldots & \ldots\end{array}$ & & $67 \cdot 50$ & cent \\
\hline $\begin{array}{llllll}\ldots & \ldots & \ldots & \ldots & \ldots \\
\end{array}$ & $\ldots$ & $12 \cdot 56$ & ," \\
\hline $\begin{array}{cccc}\text { Cell substance... } & \ldots & \ldots & \ldots\end{array}$ & $\ldots$ & $13 \cdot 28$ & \\
\hline Nitrogen-free organic matter & ... & $3 \cdot 99$ & $"$ \\
\hline Ash $\ldots \ldots$ & ... & $2 \cdot 67$ & $"$ \\
\hline
\end{tabular}

is then known as colostrum or "beestings," has a yellow or yellowish-brown colour, and the specific gravity varies between 1.040 and 1.080. Colostrum differs markedly from ordinary milk; it is thicker and it also coagulates on boiling; the so-called "calf-cheese" is obtained in this way.

1 Mycelial threads, particles of dung and lencocytes. The bacteria are scarcely visible with this enlargement. 
The chemical composition of colostrum varies chiefly from that of milk as regards the protein, for whereas a normal sample of the latter contains on an average 3.5 per cent. protein, the former, drawn immediately after calving, may have as much as 16 per cent. or more. It should be noted that this excessive quantity of protein is composed principally of albumin and globulin, and that the percentage of casein is slightly less than in normal milk.

Eugling found that the colostrum obtained during a period of 72 hours after calving had the following analyses:

Composition of Colostrum.

\begin{tabular}{|c|c|c|c|c|c|c|c|c|c|}
\hline & & & & \multicolumn{5}{|c|}{ Number of hours after calving. } & \multirow{2}{*}{$\begin{array}{c}\text { Ordinary } \\
\text { milk. }\end{array}$} \\
\hline & & & & $\begin{array}{c}\text { Immedi- } \\
\text { ately } \\
\text { after. }\end{array}$ & $\begin{array}{c}10 \\
\text { hours. }\end{array}$ & $\begin{array}{c}24 \\
\text { hours. }\end{array}$ & $\begin{array}{c}48 \\
\text { hours. }\end{array}$ & $\begin{array}{c}72 \\
\text { hours. }\end{array}$ & \\
\hline $\begin{array}{l}\text { Water... } \\
\text { Casein... }\end{array}$ & $\cdots$ & $\cdots$ & & $73 \cdot 17$ & $78 \cdot 77$ & $80 \cdot 63$ & $85 \cdot 81$ & $\begin{array}{r}86 \cdot 64 \\
3.33\end{array}$ & $\begin{array}{r}87 \cdot 75 \\
3.00\end{array}$ \\
\hline Albumin & $\cdots$ & $\cdots$ & $\dddot{j}$ & $16 \cdot 56$ & $0 \cdot 29$ & 6.95 & 9.31 & $1 \cdot 03$ & 0.50 \\
\hline Globulin & ... & .. & \} & 10.00 & 932 & 6.25 & $2 \cdot 31$ & 1.03 & 0.50 \\
\hline Fat $\ldots$ & ... & $\ldots$ & ... & $3 \cdot 54$ & $4 \cdot 66$ & $4 \cdot 75$ & $4 \cdot 21$ & $4 \cdot 08$ & $3 \cdot 40$ \\
\hline Milk sugar & r... & ... & ... & $3 \cdot 00$ & $1 \cdot 42$ & $2 \cdot 85$ & $3 \cdot 46$ & $4 \cdot 10$ & $4 \cdot 60$ \\
\hline Ash $\quad \ldots$ & $\ldots$ & $\ldots$ & $\ldots$ & $1 \cdot 18$ & $1 \cdot 55$ & $1 \cdot 02$ & 0.96 & 0.82 & $0 \cdot 75$ \\
\hline
\end{tabular}

The large amount of albumin and globulin in the colostrum, it is easy to see, is the cause of its coagulating on heating. Of the fat which can be extracted from colostrum 13.8 per cent. comes from the cholesterin and 8 per cent. from the lecithin. The sugar which is found in colostrum is not entirely milk sugar, but part of it is dextrose, and possibly some other variety of sugar is also present.

Under the microscope colostrum can easily be distinguished from normal milk by the presence in it of characteristic colostrum bodies (corps granuleux of Donné).

These little bodies are leucocytes, which have taken up a large number of minute drops of fat, so that they have increased considerably in size, and have become irregular in shape in some cases, as is seen in Fig. 3.

After a few (4-5) days the colostrum passes into milk of normal appearance and composition, for it has then fulfilled its 
purpose, which was to provide the newly-born calf with a suitable form of nutriment.

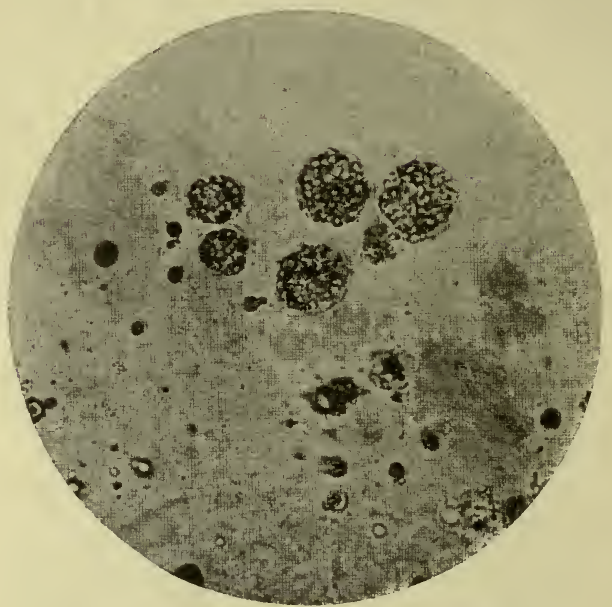

FIG. 3. - Microphotograph of Colostrum. ${ }^{1}$ The fat corpuseles are stained black with osmic acid.

In the following table are given, for comparison, the analyses of the milk of different animals, and of woman. 'These figures are taken from recent investigations, and are the averages of a great many analyses which have been made of the various milks.

Average Composition of Human Milk and that of Different Animals.

\begin{tabular}{|c|c|c|c|c|c|c|c|c|c|}
\hline & 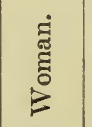 & $\dot{\vec{\theta}}$ & 完 & 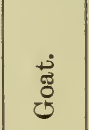 & 离 & 芯 & 离 & 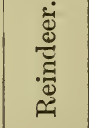 & $\frac{\dot{0}}{\tilde{E}}$ \\
\hline 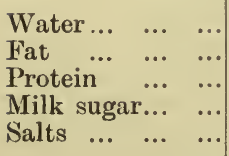 & $\begin{array}{r}85 \cdot 32 \\
3 \cdot 43 \\
1 \cdot 55 \\
6 \cdot 44 \\
0 \cdot 26\end{array}$ & $\begin{array}{r}87 \cdot 75 \\
3 \cdot 40 \\
3 \cdot 50 \\
4 \cdot 60 \\
0 \cdot 75\end{array}$ & $\begin{array}{r}82 \cdot 57 \\
7 \cdot 63 \\
4 \cdot 69 \\
4 \cdot 30 \\
0 \cdot 81\end{array}$ & $\begin{array}{r}86 \cdot 34 \\
4 \cdot 25 \\
4 \cdot 40 \\
4 \cdot 26 \\
0 \cdot 75\end{array}$ & $\begin{array}{r}81 \cdot 08 \\
7 \cdot 67 \\
6 \cdot 08 \\
4 \cdot 26 \\
0 \cdot 91\end{array}$ & $\begin{array}{r}90 \cdot 38 \\
1 \cdot 00 \\
1.98 \\
6 \cdot 28 \\
0 \cdot 36\end{array}$ & $\begin{array}{r}90 \cdot 30 \\
1 \cdot 30 \\
1 \cdot 80 \\
6 \cdot 20 \\
0 \cdot 40\end{array}$ & $\begin{array}{r}67 \cdot 7 \\
17 \cdot 1 \\
10 \cdot 9 \\
2 \cdot 8 \\
1 \cdot 5\end{array}$ & $\begin{array}{r}60 \cdot 47 \\
20 \cdot 00 \\
12 \cdot 42 \\
5 \cdot 63 \\
1 \cdot 48\end{array}$ \\
\hline & $100 \cdot 00$ & $100 \cdot 00$ & $100 \cdot 00$ & $100 \cdot 00$ & $100 \cdot 00$ & $100 \cdot 00$ & $100 \cdot 00$ & $100 \cdot 00$ & $100 \cdot 00$ \\
\hline Specific gravity .. & $1 \cdot 032$ & $1 \cdot 0315$ & $1 \cdot 033$ & $1 \cdot 033$ & $1 \cdot 038$ & $1 \cdot 034$ & $1 \cdot 033$ & - & - \\
\hline
\end{tabular}

1 After a microphotograph by Prof. C. O. Jensen, Copenhagen. 


\section{The Examination of Milk.}

\section{A. Sampling.}

In the taking of a sample of milk for the purposes of analysis or investigation, certain precautions must be observed owing to the fact that the larger fat globules immediately begin to rise to the surface even when the milk has only stood for a short time. The chief point in taking an average sample which shall faithfully represent the bulk of the milk is to subject the latter to a thorough stirring immediately before drawing the sample. The way in which such a stirring can best be performed will depend upon the amount of milk and the shape and size of the

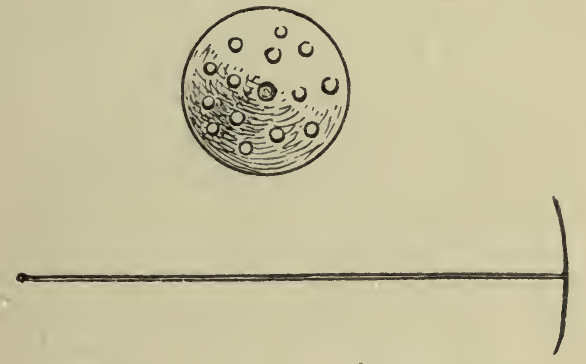

FIc. 4. - "Rouser" for Mixing Milk.

vessel in which it is contained. If the milk is in an ordinary railway can or churn, it is most satisfactory to use a so-called "rouser," which consists of a round sheet of tin-plate in which a number of holes, about three-quarters of an inch in diameter, are punched.

This circular piece of tin, which must be somewhat smaller than the neck of the churn, is fastened to a long iron shaft, and by slowly raising and lowering the "rouser" through the milk a thorough mixing is assured. Immediately after the milk has been mixed a sample must be taken, and the way in which this is done depends upon the vessels in which the milk is contained. If the milk is in several churns of different sizes, and a collective sample has to be taken, then the samples drawn from the various churns must bear some relation to the size of these vessels. For example, from a can containing 50. 
$\mathrm{kg}$. of milk a sample of half a litre would be taken, and from one containing $30 \mathrm{~kg}$., 300 c.c., and so on. These different samples would then be thoroughly mixed in some convenient vessel, and from this the main sample would be drawn. It is more satisfactory, of course, to empty the contents of the various cans or churns into a suitable vessel, and then, after a thorough stirring, to take out the sample which is to be tested.

Often enough it happens that the milk from which a sample is to be drawn is allowed to stand so long that a thick layer of cream, which does not easily mix again with the rest of the milk, forms on the surface. In such a case it is only necessary to warm the milk to about $40^{\circ} \mathrm{C}$. by placing the vessel in which it is contained into hot water, for afterwards it is easy to effect a thorough mixing.

When taking samples of milk from individual cows during test milkings, a course which must be adopted if the milk production of each animal is to be ascertained, then it is best to use an apparatus similar to the one described below.

The milk from each cow is weighed in the cylindrical vessel, either on a weighing machine similar to the one shown in Fig. $5 a$ or on a spring balance, and then the glass tube shown on the right of the figure is sunk to the bottom of the vessel. The upper end of the glass tube is then closed with the finger so that the milk cannot flow out, and the sample is in this way transferred to one of the numbered bottles, one of which is supplied for each cow. Similarly, at the other times of the day when the cows are milked, a sample is taken, and the two or three individual samples make a collective sample for the day's milk.

If the sample of milk is to be tested at once on the spot, no special precautions are needed to preserve it; but if, on the other hand, one or more samples have to be forwarded to a laboratory, this should be done in well-corked bottles, which must be clean and dry before the milk is put into them. If only the percentage of fat in the milk is to be determined, then 100 c.c. will suffice; but if a fuller investigation is to be made, then a larger quantity, say 500 c.c., should be taken. In no circumstances, though, should the sample bottles be quite full, for, if so, it would hardly be possible to get the cream, 'which had separated out, back again into its normal state in 
the milk. The bottles, therefore, must never be filled more than three-quarters full.

If a sample of milk is to be analysed for legal purposes, then
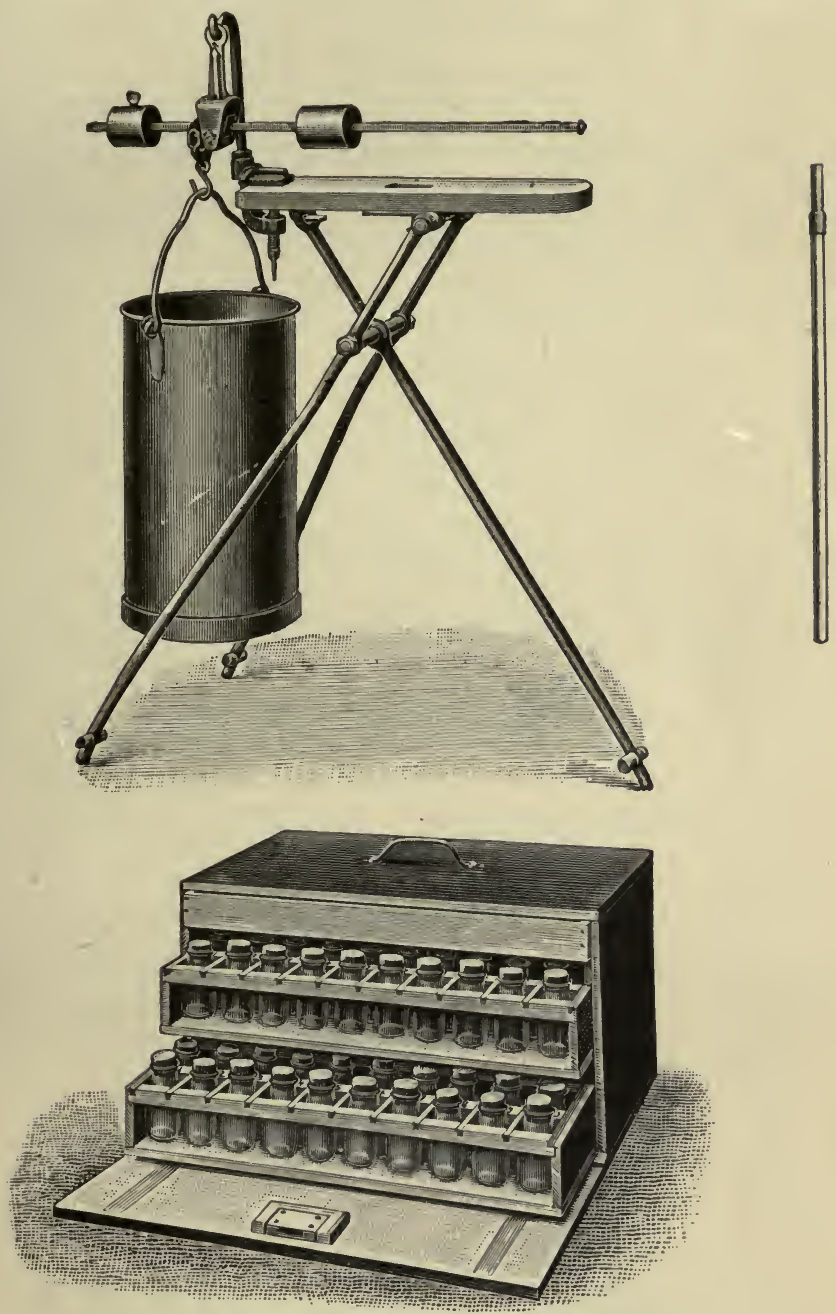

Figs. $5 a$ and 5b.-Apparatus for Test Milkings.

it must be taken in the presence of witnesses, sealed up, and a written statement made as to the owner of the milk, time, place, and method of sampling, \&c. In sending milk samples for 
analysis it is always advisable to add some sort of preservative, for it is never certain if the samples can be analysed immediately on arrival at the laboratory. Many different substances have been used as preservatives, but only a few have shown themselves to be really suitable for the purpose. In choosing such a preservative material it must not be forgotten that it has not only to protect the milk against changes due to micro-organisms, but it must also cause no alteration in its chemical composition.

It will suffice to mention here the most important substances used for the preservation of milk samples. In Europe, potassium bichromate, $\mathrm{K}_{2} \mathrm{Cr}_{2} \mathrm{O}_{7}$ and formaldehyde, $\mathrm{CH}_{2} \mathrm{O}$, are generally employed. Potassium bichromate is a very suitable preservative, and was first brought forward by Dr. J. E. Alén, of Gothenburg. Bichromate has the advantage of not in any way altering the chemical composition of the milk, and although it is poisonous, still the yellow colour which it imparts to the milk prevents the sample being inadvertently drunk. About as much bichromate as will lie on the point of a knife $(0.5 \mathrm{~g}$. to 1 litre of milk) is sufficient to preserve the sample for several weeks, provided the temperature does not rise very high. There is, however, one drawback to the use of bichromate, and that is it causes the cream to rise more quickly than it would normally. This is due to the fact that the dissolved salt raises the specific gravity of the milk, so that a greater difference arises between the specific gravity of the milk fat and that of the milk serum. The cream, therefore, gathers in a thick layer; but if the milk is warmed to about $40^{\circ} \mathrm{C}$., then, as previously mentioned, it is not difficult thoroughly to mix the cream with the milk again.

For the preservation of small samples of milk a concentrated solution of bichromate may be used, and one drop measured from a dropping bottle into each 10 c.c. of milk: If the bottles are sent from the laboratory to the place where the samples of milk are to be taken, then it is preferable to put the requisite quantity of bichromate into them before they leave.

If the preserved milk has to be tested for specific gravity, dry matter, and ash, then potassium chromate cannot of course be used, unless, perhaps, in the case of the dry matter, where the chromate can be specially estimated according to the method given on p. 90, and the amount deducted from the total weight. In such a case as this, however, it is preferable to use form- 
aldehyde as a preservative. Generally a 40 per cent. solution is employed, which is known commercially as formalin; 1 c.c. of this is sufficient to preserve a litre of milk for a long time. For smaller quantities of milk it will be found that 50 c.c. are adequately preserved by one drop of formalin.

Formaldehyde, however, renders the protein substances in the milk very difficultly soluble, and this is of disadvantage in estimating the fat by Gerber's method. The undissolved protein forms a plug below the column of fat in the butyrometer, and makes an exact reading difficult. This condition only arises, however, if relatively large quantities of formalin ( 5 per thousand or more) are used.

Before the bottle containing a sample of milk which is to be investigated is opened, it must be repeatedly shaken to mix the cream, which has separated on the surface, with the milk below. If this is not easy to do, then the bottle should be warmed to $40^{\circ} \mathrm{C}$. in a water-bath, after which the two liquids in the bottle will quickly mix on shaking. The bottle should then be opened and the contents emptied into a large clean beaker; if any cream is left adhering to the inside of the bottle the milk must be poured back again, and the bottle shaken until no traces of cream are to be seen. Immediately before taking a known quantity of milk by means of a pipette, the contents of the beaker should be poured into another clean, dry beaker, and back again, to ensure a thorough mixing. When no preservative has been added to the sample of milk it often happens that it arrives for investigation in a sour or coagulated condition. The following method, proposed by Dr. M. Weibull,, of Alnarp (Sweden), can then be employed, and it serves also in the investigation of samples of sour buttermilk.

The milk which has become sour is not poured from the bottle, but a rough estimate is made of the quantity which is there. Suppose that the estimated amount be taken as 200 c.c.; to this 20 c.c. of ammonia, that is, 10 per cent. of the sour milk, is then added from a burette, the cork replaced, and the bottle thoroughly shaken until the coagulated material dissolves in the ammonia. The result ought to be a homogeneous, slightly opaque liquid. The contents of the bottle are then emptied

1 Kgl. Landtlruks-Akad. Handl. o. Tidskr., 1894, p. 243; Milch-Zeitung, 1894, pp. 247 and 413. 
into a measuring cylinder and exactly measured; suppose in this case the volume to be 205 c.c., if from this the $20 \mathrm{cc}$. of added ammonia be deducted, there remains 185 c.c. as the amount of milk originally present in the bottle. Specific gravity, percentage of fat, \&c., can then be determined in the usual way in this mixture of milk and ammonia, and the results calculated to the original volume of milk.

This method cannot, however, be applied if the coagulated milk is very old, for in sour whole milk the fat gradually undergoes decomposition, so that an estimation of the fat then becomes unreliable. Separated milk and buttermilk, on the other hand, are much more stable, and it is possible with the help of Weibull's method to analyse very old samples of these two sorts of milk with good results.

\section{(B.) Physical Examination.}

The purely external indications that milk is normal and fresh are its appearance, taste, and smell.

In appearance the milk must be a completely homogeneous, opaque liquid which, on standing, must give a homogeneous and sharply defined layer of cream.

The presence of flocculent matter in the milk points either to some disease in the cow, such as inflammation of the mammary gland, or to the milk being so old that changes due to micro-organisms have taken place. The colour of milk should be that of porcelain; it can, however, have either a yellowish or a bluish finge, according to the amount or colour of the contained fat. A reddish tinge is indicative of blood from the udder, from which it may be concluded that this organ has been injured in some way or the other. In exceptional cases the red colour may come from such foods as mangels, beets, carrots, \&c., or from colostrum milk.

Milk should have a pleasant, characteristic, sweet taste; any sourness indicates an advanced stage of lactic acid fermentation.

A bitter or salty flavour is sometimes found in milk from cows which are practically dry or are suffering from some disease of the udder. It may arise also from the action of peptonising bacteria upon the protein of the milk, whereby substances (peptone) with poisonous properties are formed. 
Certain feeding stuffs impart a characteristic and often strong flavour to milk, as, for instance, mangels, turnips, \&c. Rusty and untinned vessels give a very objectionable metallic taste to the milk.

The smell of milk should not be at all strong; a faint animal odour is, however, always to be noticed. Milk very easily acquires the smell and taste of its surroundings; if, for instance, the stall is badly ventilated, the flavour and smell of dung are soon imparted. These unpleasant properties can also arise from the entrance of particles of dung during careless milking. If the cowshed is disinfected with such strongsmelling substances as carbolic acid, lysol, \&c., the milk will certainly be tainted with the characteristic odour of these disinfectants.

A sour smell indicates that the milk is not fresh, and this smell is noticeable before the milk is sour to the taste. It is a sign that the milk sugar is going to be converted into lactic acid under the influence of micro-organisms.

\section{Determination of the Specific Gravity of Milk.}

A determination of the specific gravity of milk must not be made directly after milking, for the specific gravity of freshly drawn milk increases during the five hours following milking. The difference between the specific gravity of fresh milk and that of the same five hours later is about 0.001 . This phenomenon has not yet been satisfactorily explained; it is possible that the cause lies in the changes that the casein, which is present in a colloidal form, undergoes in the first five hours.

The instrument generally used for the determination of the specific gravity is the lactometer, one of the most satisfactory types of which is from the design of Soxhlet, constructed by Joh. Greiner of Munich. ${ }^{1}$

This lactometer, sometimes called a milk aräometer or milk hydrometer, has a divided scale reading from 1.024 to $1 \cdot 038$, the distance between each division being $7.5 \mathrm{~mm}$. Between the main divisions are shorter ones corresponding to half a degree, so that it is not difficult with the eye to read to a tenth of

${ }^{1}$ Neuhauser Strasse, No. 49. 
a degree. The scale of the lactometer is graduated for a temperature of $15^{\circ} \mathrm{C}$., so that at that temperature the specific

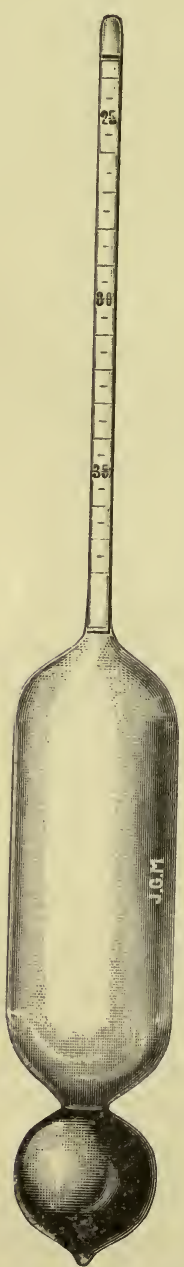

FiG. 6A.-Soxhlet's Lactometer without Thermometer.

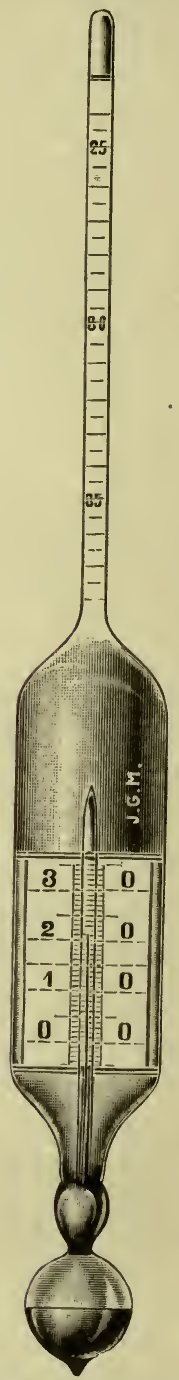

Fig. 6B.-Soxhlet's Lactometer with Thermometer.

gravity of milk compared to that of water at $15^{\circ} \mathrm{C}$. is obtained.

The lactometer is often provided with a thermometer, which 
is either fixed to the lower wider part of the lactometer or else in an extension of the stem.

In France and Switzerland, Quevenne's lactometer is largely used, and it also is sometimes provided with a thermometer. The scale of Quevenne's lactometer has more divisions than Soxhlet's, so the space between two divisions is less, and the difficulty of taking a very accurate reading is greater.

There are many different sorts of lactometers to be bought, only few of which, however, are really reliable. Great care should be exercised when buying a lactometer to get an accurately-adjusted instrument.

When making a determination of the specific gravity of milk, it is not essential to have the temperature exactly at $15^{\circ} \mathrm{C}$. It is sufficient if it lies between $10^{\circ}$ and $20^{\circ} \mathrm{C}$., for with the help of the table drawn up by Chr. Müller (Table I in Appendix) the specific gravity obtained at temperatures between these two can easily be calculated to $15^{\circ} \mathrm{C}$.

The milk which is to be tested should be well mixed and enough poured into a glass cylinder, which must not be too narrow, to fill it two-thirds full. If this is done carefully, then no foam will be formed on the surface, but should there be any it must be removed in some suitable way, e.g. by filter paper. The lactometer, which must be clean and dry, is held by the stem and carefully lowered into the milk until the surface of the liquid is at division 30 on the seale $(1.030)$. It is then freed, and when it has come to rest the height of the milk on the scale is read off, care being taken to have the eye on the same level as the surface of the milk. Around the stem of the lactometer the level of the milk is slightly raised owing to adhesion. This must be allowed for in the reading; as a rule it is about $\frac{1}{10}$ th of a degree. If, for instance, $30 \cdot 2$ has been read off on the scale, then 0.1 must be added, making 30.3 (1.0303).

Each time after it has been used, the lactometer must be washed under the water-tap and then carefully dried, for if it is left unwashed the milk will dry on to the glass and be difficult to get off.

For very accurate determinations, as in scientific investigations, it is safer to use a pyknometer or a Westphal balance. The construction of these instruments and the method of using them are well known to chemists, so will not be dwelt upon 
here. When buying a lactometer its accuracy should first of all be tested against a pyknometer or a Westphal balance.

As has been mentioned (p. 22), the specific gravity of sour, coagulated milk can be determined by means of Weibull's method. The formula which Weibull uses for the mixture of milk and ammonia is :

$$
S=\frac{(M+A \cdot) S^{\prime}-A \cdot S^{\prime \prime}}{M}
$$

Where

$$
\begin{aligned}
& S=\text { sp. gr. of the milk. } \\
& S^{\prime}=" \quad " \quad \text { milk-ammonia liquid. } \\
& S^{\prime \prime}=\quad " \quad " \text { ammonia. } \\
& M=\text { the volume of milk in c.c. } \\
& A=\quad " \quad \text { ammonia in c.c. }
\end{aligned}
$$

The most important application of the specific gravity of milk is when testing for the addition of water or the removal of cream. A return, therefore, will be made to this subject in the section dealing with milk adulteration

\section{Estimation of the Dirt.}

The estimation of the amount of dirt, that is, the solid impurities, in milk, is of importance hygienically. F. Renk was the first to show the value of a quantitative determination of the dirt in milk. ${ }^{1}$ A fairly satisfactory method for carrying out Renk's principle has been proposed by A. Stutzer. ${ }^{2}$

Stutzer's apparatus consists of a bottle of the usual shape, capable of holding 1 litre. An ordinary test-tube, without the usual turned-back edge, is connected to the neck of the bottle by means of a piece of rubber tubing. Before fitting the rubber tubing, to one end of which the test-tube is attached, on to the neck of the bottle, the latter is filled with 1 litre of the milk which is to be examined. Then the free end of the rubber tubing is fitted on to the bottle, and the latter inverted and held in this position by means of a retort stand and ring, the test-tube now occupying the lower position. After 1 or

1 Verhandl. des $X$ internat. med. Kongr., Berlin, 1891, Vol. V, p. 164; also Milch-Zeitung, 1893, p. 594, and 1899, p. 65; further, Molkerei-Zeitung, Berlin, 1898, p. 405.

2 Die Milch als Kindernahrung, dc., Bonn, 1895, p. 5. 
2 hours the solid impurities in the milk have sunk downwards and collected on the bottom of the test-tube. With the aid of a clip the rubber connection between the bottle and the testtube is closed, the latter freed, and the majority of the milk carefully removed with a pipette. Distilled water is then filled into the test-tube, and, after well shaking, the contents of the tube are poured on to a dry weighed filter, and the residue washed with water until no trace of opalescence is to be seen in the filtrate. The solid matter on the filter is then washed repeatedly with alcohol and ether, dried and weighed.

Very good and rapid results were obtained by Ballo ${ }^{1}$ by filtering the milk, without previous sedimentation, through filters of very fine gauze made like ordinary filters. The gauze should be of the finest, No. 18 or 20 (Dufour), and this will retain all the particles which are visible to the eye but will permit the largest fat globules to pass through. These gauze filters are placed in a ribbed funnel, moistened with water, and the milk poured in. This quickly passes through, leaving the dirt, which can be further treated, as in Renk's method, by washing with water, alcohol, and ether, and then weighing. The above methods, with unimportant modifications, are those generally employed in a determination of the dirt in milk; they are slow, but up to the present a rapid quantitative method is not known. Instead of washing with pure water, Rosengren recommends the addition of $\mathrm{HCl}$, for otherwise there is danger of the calcium phosphate, which is a normal constituent of milk, being deposited in the dirt, and so the weight of the latter would be too high. The calcium phosphate and similar compounds are soluble in water containing $\mathrm{HCl}$.

Some of the more important modifications of Renk's method may fitly find a place here. Bersch advocates the addition of formalin to the milk, so that it does not quickly turn sour, and the time of sedimentation can then be lengthened to 24 hours. Winkler would like to employ 8-10 litres of milk instead of 1 litre. This certainly would be of advantage in many cases, for the amount of dirt from 1 litre of cleanly-handled milk is often minute and difficult to work with.

Gerber has brought out a modification of Stutzer's apparatus (p. 26), in which the bottom of the bottle is removed and the

${ }^{1}$ Milch-Zeitung, 1904, p. 229. 
connection between the bottle and the test-tube can be closed by a plug made from a rubber cork and a long piece of iron wire. The test-tube is also modified, being drawn out into a narrow prolongation at the bottom, which admits of an estimation of the amount of dirt being quickly made. The figure below gives a view of this piece of apparatus, which, however, has one drawback in that the bottles only hold half a litre, which is too little if a reliable figure is to be obtained for the amount of dirt present.

An apparatus which may perhaps supplant those described above is due to Späth, and, as will be seen in Fig. 8, the sedi-

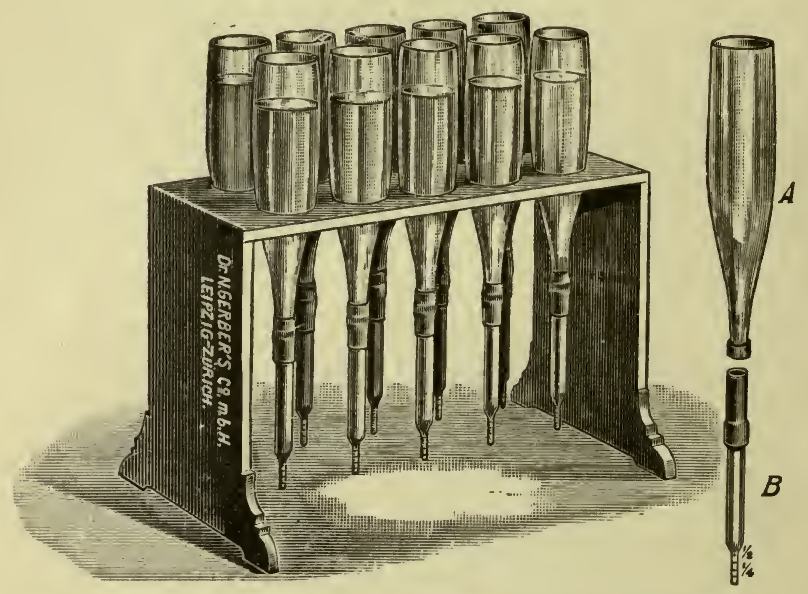

FIG. 7.-Gerber's Apparatus for Estimating Dirt in Milk.

mentation takes place in a conical vessel or funnel which holds 1 litre of milk. The dirt collects in a cavity made in the glass tap at the bottom of the funnel, so that when all has settled it is only necessary to turn the tap and pour the milk out of the funnel. After careful washing of the funnel, the tap is turned again to its original position, distilled water poured in, and the dirt again allowed to settle. When this operation has been repeated several times the contents of the cavity are washed out on to a weighed filter, and the residue treated with alcohol, ether, \&c., as mentioned previously.

It is absolutely essential that no sharp projections or crevices should be present in any of the pieces of apparatus mentioned 
above, for otherwise particles of dirt could not sink to the bottom. The figures which are obtained for the amount of dirt in milk ought not to have too much importance attached to them. A not unimportant part of the original dirt dissolves in the milk, and so escapes; another portion adheres to the particles of fat, and is carried away with them instead of sinking with the other sediment. There is some further loss, too, during the treatment with alcohol and ether on the filter.

The determination of the dirt has, however, a relative value, for it serves as an indication of the care with which the milk has been handled. According to Renk, 1 litre of good, pure milk ought, after standing two hours in a vessel with transparent bottom, to show no traces of deposit.

Quantitatively, a milk which has been carefully and cleanly obtained will contain 3-10 mg. dirt per litre, whilst those samples which are taken in the ordinary way of trade often contain considerably more. The amount of dirt in a milk should be expressed in $\mathrm{mg}$. per litre.

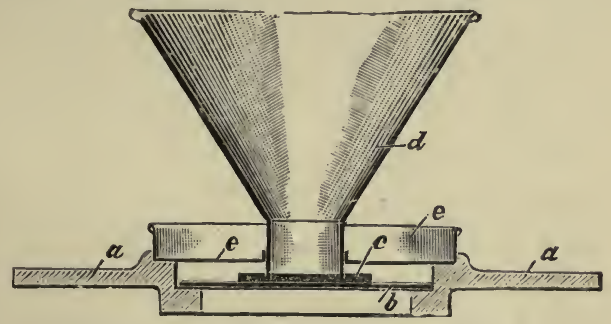

Fia. 9.-Bernstein's Dirt Tester.

If there is no need to make a quantitative estimation of the dirt in a sample of milk, then, by filtration through a thin layer 
of cotton wool, it is possible to compute with fair accuracy how much dirt is present. Fliegel, Bernstein, and Gerber have constructed various forms of apparatus for this purpose.

In Bernstein's apparatus (Fig. 9) the milk passes through a small cotton-wool filter, and by simple observation of the filter an idea of the quantity of dirt in the milk can be obtained.

\section{Chemical Examination.}

\section{Determination of the Acidity of Milk.}

A knowledge of the degree of acidity of a sample of milk is of great importance in judging of its freshness or its suitability for some particular purpose, such as the making of a certain sort of cheese. Further, it is important to determine the acidity of sour cream in order to control the process of ripening.

The following methods are especially worth attention when a determination of the degree of acidity of milk has to be made.

\section{The Reaction of the Milk to Litmus Paper.}

Normal, fresh milk has an amphoteric reaction: that is, it turns blue litmus paper feebly red, and red litmus paper blue. A distinctly acid reaction to litmus, therefore, indicates a milk in which the normal degree of acidity has been passed, whilst a predominating alkaline reaction shows that the milk is abnormal, and may either have come from diseased animals or have been mixed with water.

\section{The Boiling Test.}

If the milk coagulates on being heated in a test-tube, it is an indication that it is already strongly acid. Normal milk coagulates on boiling when it contains about $0.26 \%$ lactic acid; this is equivalent, according to Soxhlet-Henkel (see below), to 12 c.c. $N / 4 \mathrm{NaOH}$ per 100 c.c. of milk.

\section{The Alcohol Test.}

Equal quantities of milk and alcohol (60 per cent. by weight or 68 per cent. by volume) are mixed in a small test-tube. 
Then the test-tube is inclined so that the mixed liquid covers a portion of the side. On slowly raising the tube to the vertical position it should be noted whether any white flakes adhere to the glass, for if so the milk is strongly acid. A slight film indicates a small degree of acidity, whereas with fresh milk the side of the tube which has been moistened with the liquid remains elear.

The three methods given above only allow of a rough estimate of the degree of acidity being made. When an accurate determination is required, titration against an alkali must be made. This may be done in several ways, and the most important methods are the following:

\section{Soxhlet-Henkel ${ }^{1}$ Method of Titration.}

This is the oldest method and the one generally used in practice. Fifty c.c. of milk, to which 2 c.c. of a 2 per cent. alcoholic solution of phenolphthalein have been added, are titrated with $N / 4 \mathrm{NaOH}$ until a faint red colour is perceptible. The number of cubic centimetres of caustic soda which are necessary is termed the degree of acidity. In this method no water is added to the milk.

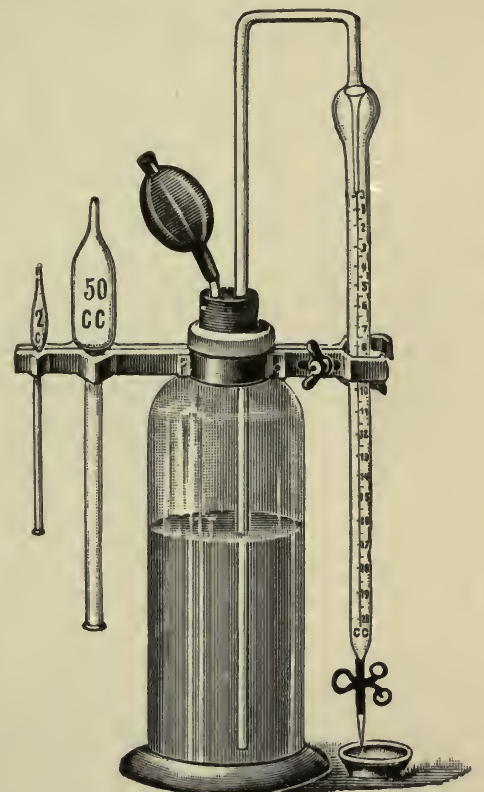

FIG. 10. - Soxhlet-Henkel Titration Apparatus. According to Soxhlet, fresh cows' milk should have, on an average, 3.5 degrees of acidity. Milk which coagulates on boiling has, as already mentioned, about 6 degrees, whilst milk which has become sour and coagulated of itself usually has about 15 degrees. Good ripened cream for butter-making should have an acidity of 12-16 degrees. The figure given above shows an apparatus designed for the titration, the burette being automatically regulated to fill to the zero point.

1 Chemisches Centralblatt, 1887, p. 229. 
If the amount of lactic acid corresponding to the degrees of acidity is required, then it is only necessary to multiply by 0.0225 to get the lactic acid expressed in grams.

In a later method by Soxhlet-Henkel, 100 c.c. of milk are titrated with $N / 4 \mathrm{NaOH}$ and 4 c.c. phenolphthalein solution, and the number of cubic centimetres then gives the degree of acidity. It is necessary to know, when dealing with the "degrees of acidity according to Soxhlet-Henkel," whether the titration has been made with 50 or 100 c.c. of milk.

\section{Thörner's ${ }^{1}$ Method of Titration.}

Ten c.c. are diluted with 20 c.c. of distilled water, 5 drops of a 5 per cent. alcoholic phenolphthalein solution added, and the titration with $N / 10 \mathrm{NaOH}$ carried out in the usual way. The number of cubic centimetres of $N / 10 \mathrm{NaOH}$ which are required, multiplied by ten, expresses the degree of acidity for 100 c.c. milk. If, for instance, $1 \cdot 7$ c.c. of $N / 10 \mathrm{NaOH}$ are required to neutralise 10 c.c. of milk, then the milk has 17 degrees of acidity.

This method is often preferred to the older Soxhlet method because the quantity of milk required is less, and there is no need to prepare a special $N / 4 \mathrm{NaOH}$ solution; the ordinary $N / 10 \mathrm{NaOH}$, which is always at hand in the laboratory, can be used.

Thörner's method, however, has the disadvantage that the dilution with water causes a loss of acidity, for some of the alkaline calcium phosphate is brought into solution by the water. This, of course, may be avoided by not adding water to the milk.

Fresh milk has an average acidity of 16-18 degrees by Thörner's method; at 25 degrees and above the milk coagulates on boiling.

By multiplying the number of cubic centimetres of $N / 10 \mathrm{NaOH}$ by 0.009 , the amount of lactic acid in grams is obtained.

\section{Dornic's ${ }^{2}$ Method of Titration.}

Dornic uses a caustic soda solution which is somewhat stronger than decinormal ; it contains $4.444 \mathrm{~g}$. NaOH per litre. Nine

1 Chemiker-Zeitung, 1892, Nos. 80 and 83 ; Milch-Zeitung, 1893, p. 58.

2 Le Contrôle du Lait: Besançon, 1897. 
c.c. of Dornic's solution are equivalent to 10 c.c. $N / 10$ acid. As the molecular weight of lactic acid is $90,0 \cdot 1$ c.c. of Dornic's solution is exactly equal to $1 \mathrm{mg}$. lactic acid. Dornic has constructed a special burette so graduated that one division $=0.1$ c.c., so that the amount of lactic acid in milligrams can be directly read. The number of burette divisions of caustic soda solution required to neutralise 10 c.c. of milk is known as the degrees of acidity, according to Dornic, of that particular milk. As indicator, 5 drops of a 2 per cent. alcoholic solution of phenolphthalein are used.

Fresh cow's milk has an acidity of 16-20 degrees (Dornic).

\section{Determination OF THE Dry MatTer or TOTAL SOLIDS.}

To determine the dry matter in milk, a weighed quantity of it is dried at $100^{\circ} \mathrm{C}$. until the weight is constant. The loss of weight represents the water which has evaporated, the remaining weight is that of the dry matter.

The desiccation of the milk is usually done in a basin containing ignited sand or pumice stone. In recent times the flat nickel basins advocated by Soxhlet are much used. The basin with the sand is first weighed after the whole has been dried for an hour at $100^{\circ} \mathrm{C}$. in the drying-oven. About 10 c.c. of the milk under investigation are put into a small beaker provided with a watch-glass for a cover. After the beaker and the contained milk have been weighed together, the latter is poured on to the sand in the basin, and the empty beaker again weighed. The difference between the two weights gives the weight of milk used.

The basin is then placed in the water-oven in which the drying, which generally takes several hours, is to be done. Under no circumstances must the drying be regarded as complete until two successive weighings give the same result.

With the aid of Soxhlet's glycerine drying-oven (Fig. 11) the milk can be dried more rapidly, for this apparatus is heated at $105^{\circ} \mathrm{C}$., and a strong current of air passes through it. In some laboratories a vacuum drying-oven is used for the determination of water in milk.

According to Fleischmann ${ }^{1}$ a loss of dry matter can arise

1 Lehrbuch der Milchwirthschaft. Fourth Edition. Leipsic, 1907. 
when the milk is fairly old, that is to say, when its composition has begun to alter. Fleischmann has also shown that in parallel tests, the dry matter can vary \pm 0.15 per cent. The reason of

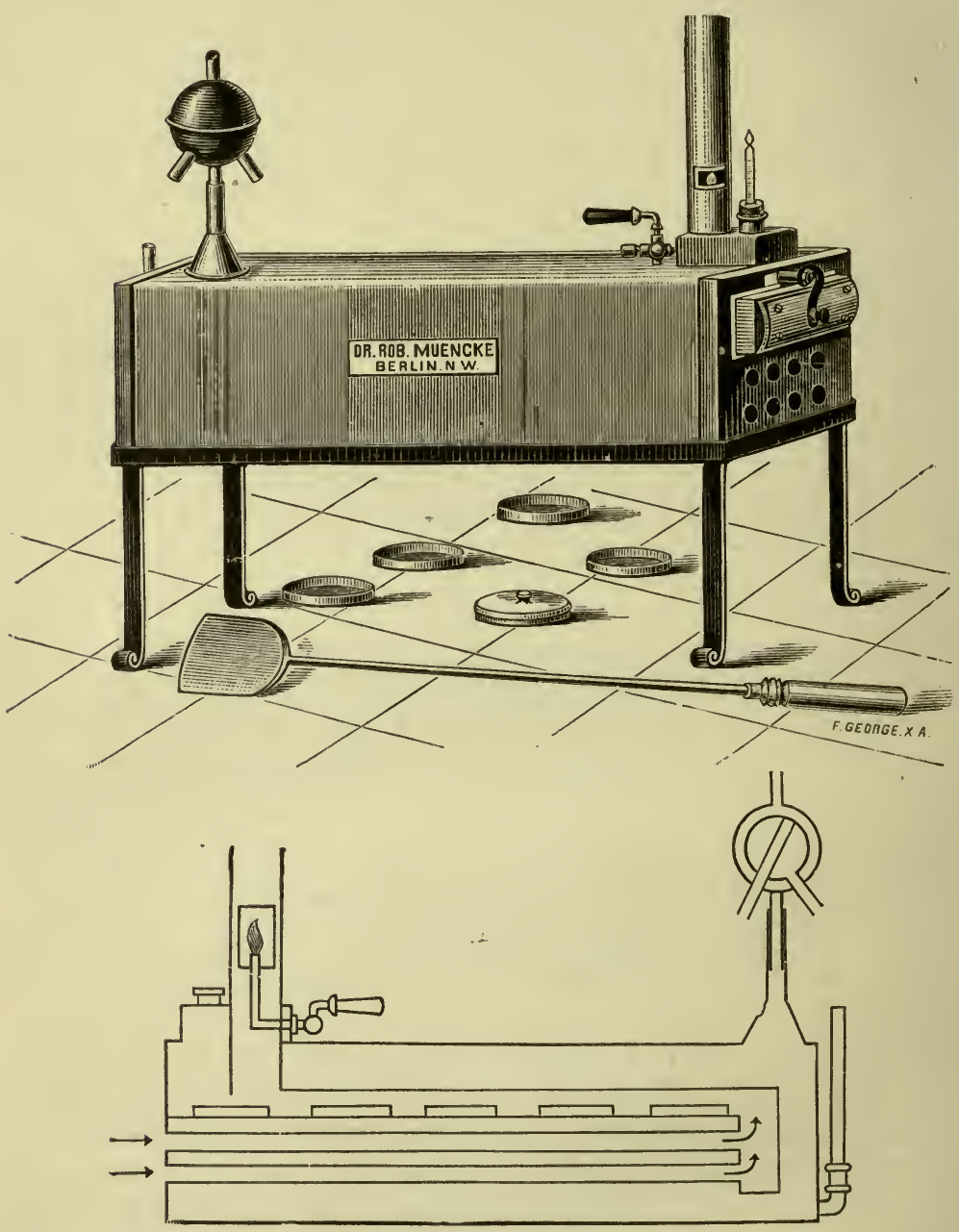

'Fıc. 11.-Soxhlet's Drying-Oven.

this is that at $100-105^{\circ} \mathrm{C}$. the milk sugar undergoes decomposition and becomes brown in colour. The allowable experimental error in a determination of dry matter of milk lies about 0.15 per cent. It is not essential to dry the milk on 
sind, punice, \&c.; Janke ${ }^{1}$ has shown that without any such material the same results are obtained.

Fleischmann has worked out a formula with the help of which, if the specific gravity and the fat contents are known, the total solids can easily be calculated. The results which are obtained in this way are very exact, and so this method can also be used to check the direct determination of the total solids. Mention will be again made of this formula in the section dealing with the adulteration of milk.

In general, the amount of total solids in milk varies between 11 and 14 per cent., the average may be taken as 12.25 per cent., as mentioned in the Introduction.

\section{Determination of the Fat.}

The fat of milk, regarded economically, is without question the inost valuable constituent of milk. It is from this fat that butter is made, which, as is well known, is an expensive article of diet. In recent years it has become the practice for large dairies and butter factories to buy milk on the percentage of fat which it contains. This is done either by paying for the amount of fat found in the milk, or a price is agreed upon for milk with a certain percentage of fat, say $3-3.4$ per cent., and a deduction made for each $0 \cdot 1$ per cent. below 3 per cent., and a supplement given for each $0 \cdot 1$ per cent. above $3 \cdot 4$ per cent. The latter method is the more usual.

A determination of the percentage of fat in milk is also of importance in judging of the productiveness of individual cows, for the value of a cow as a productive animal must depend both upon the quantity of milk and the amount of fat it contains when a certain ration is being fed.

Further, an exact knowledge of the proportion of fat is important, because in most countries there is a legal minimum standard for the percentage of fat which milk sold to the public must contain. It is therefore easy to see that the determination of the amount of fat in a milk is looked upon as the most important branch of the examination of the constituents of milk. Not only in laboratories, but also in dairies and by

1 Repert. für analyt. Chemie, 1882, p. 33. 
owners of cows as well as milk dealers, an estimation of the percentage of fat in milk has become general.

In the course of time a great number of different methods, of varying value, have been proposed for determining the fat, many of which have now only a historical interest or have found no extensive application. It will therefore suffice here to describe those which at the present time are in general use and have been proved to give satisfactory results. The methods for the estimation of fat may be divided, according to their character and application, into two main groups: scientific methods and practical methods.

\section{A. Scientific Methods.}

\section{Soxhlet's Aræometer Method.}

This method was worked out by Prof. Soxhlet of Munich ${ }^{1}$ in the year 1879, and for many years was the only one that gave good results without having recourse to the gravimetric methods of analysis which are so difficult to perform outside the laboratory. The introduction of Soxhlet's method was the consummation of long-continued attempts to provide an exact and reliable method which could be used by anybody for the estimation of fat. In actual dairy work the method has now been replaced by others which need less sensitive apparatus, but it is still used in many laboratories, although other scientific methods will probably drive it from the field.

Shortly, the principle of Soxhlet's method is the following: When a measured amount of milk is mixed with certain quantities of caustic potash solution and ether saturated with water, there separates out afterwards an ethereal solution of fat, in which all the fat of the milk is contained.

If the specific gravity of the ether-fat solution is determined by an aræometer, then the percentage of fat in the milk can be obtained. As the difference between the specific gravities of ether and fat is very great, small differences in the amount of fat make a considerable alteration in the specific gravity of the ether-fat mixture. The assumption of course has to be made

\footnotetext{
1 Zeitschrift des landwirtschaftlichen Vereins in Bayern, 1880, p. 659, and
} 1882 , p. 18. 
that the specific gravity of milk fat is constant, and with mixed milk this is really true. In the milk of single cows, variations in the specific gravity of the fat do occur, a circumstance which effects the reliability of the method in such cases.

A determination of the fat in milk by this method is performed as follows: The milk must first be thoroughly mixed, and have a temperature of $17.5^{\circ} \mathrm{C}$, then 200 c.c. are measured by means of the pipette belonging to the apparatus into the bottle (a), which holds about 300 c.c. (see below). Then 10 c.c. of caustic potash solution, sp. gr. 1.26-1.27, are added to the milk in the bottle, and the whole well shaken. The caustic potash solution is prepared by taking 400 g. solid $\mathrm{KOH}$, dissolving it in half a litre of water, allowing this to cool, and then making up to 1 litre; the solution therefore contains 40 per cent. potash. Finally, 60 c.c. of ether, which have previously been saturated with water, are added by means of the special pipette to the contents of the bottle. The ether must previously be prepared by shaking ordinary ether with one-tenth its volume of water and pouring the clear ether into a special bottle; it should be measured out at a temperature of $16.5^{\circ}-18.5^{\circ} \mathrm{C}$. After the ether has been added to the contents of the bottle, it is closed with a rubber cork and thoroughly shaken for half a minute, the bottle being afterwards placed in a vessel containing water at $17^{\circ}-18^{\circ} \mathrm{C}$. This shaking is then repeated every half minute for a period of a quarter of an hour, the bottle being repeatedly inverted. At the end of this time the bottle is allowed to stand for a quarter of an hour, by which time a clear upper layer of ether in which the fat is dissolved will have separated. The separation of this layer is hastened by moving the bottle from side to side during the last few minutes.

It is not necessary that all the ether should have separated out, provided there is sufficient for the determination of the specific gravity by means of the aræometer, but it must be perfectly clear. In certain cases, e.g., with milk very rich in fat (4.5-5 per cent.), the separation of the ether-fat mixture takes a long time: 1-2 hours. Sometimes, although this is exceptional, the ether will not separate out clear even after standing for a long time. $\mathrm{N}$. Engstrom states that this can be avoided by adding 1:3 c.c. of glacial acetic acid before the 


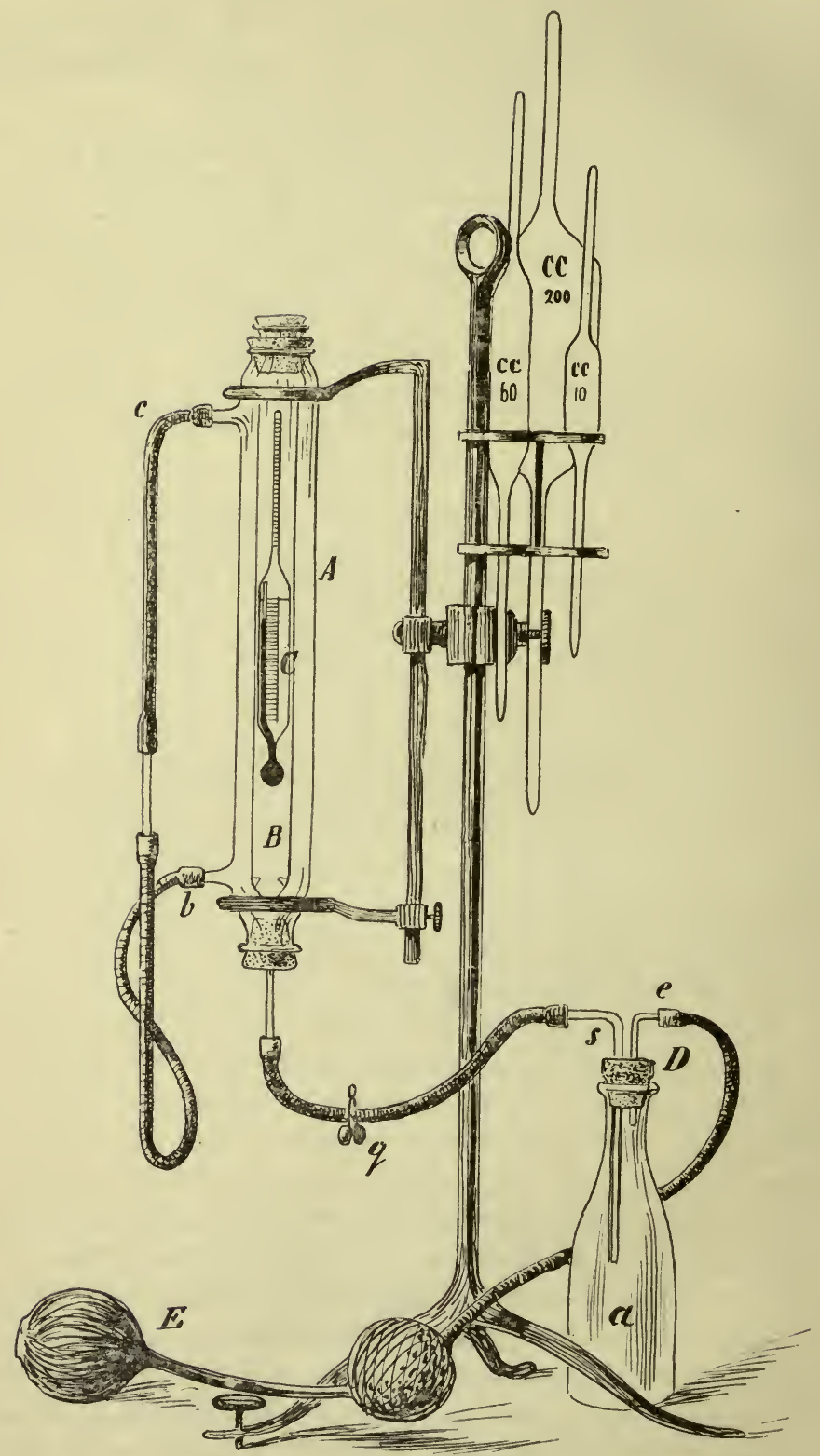

Fic. 12. - Soxhlet's Apparatus for the Estimation of Fat in Milk. 
addition of the caustic potash solution and ether. To neutralise the acetic acid, more caustic potash, 14 c.c., instead of 10 c.c., must be used.

After the fat-containing ether has separated, the water jacket $(A)$ is filled with water at a temperature of $16^{\circ}$ to $18^{\circ} \mathrm{C}$., and the bottle is then connected as shown in Fig. 12. By carefully pressing the rubber ball, the ether is forced into $(B)$, care being taken that only the clear liquid enters. The connection between the bottle and the apparatus is then closed by a pinchcock, and the small arseometer sunk into the ether. When the aræometer floats freely in the liquid, the specific gravity is read off on the scale. In order that the aræometer may freely take up its proper position, the water jacket is clamped horizontally to a retort stand, which can be carefully regulated by a screw in the foot. The temperature must also be read on the thermometer attached to the aræometer, and if it is higher or lower than $17.5^{\circ} \mathrm{C}$., a correction must be made to get the true specific gravity of the ether solution. This correction is the addition of one-tenth of a degree of the aræometer scale for each one-tenth of a degree above $17.5^{\circ} \mathrm{C}$., and a corresponding deduction of one-tenth of a degree from the specific gravity for each one-tenth of a degree below $17.5^{\circ} \mathrm{C}$. If, for example, the temperature of the ether is $16.9^{\circ} \mathrm{C}$, and the reading on the aræometer scale is $52.7^{\circ}$, then the true specific gravity is $52 \cdot 1^{\circ}$.

When the determination has been made, the pinchcock is opened and the ether flows back again into the bottle. The tube $(B)$ is then filled with ordinary ether, which is also allowed to flow into the bottle. Finally, the rubber aspirator is fixed to the lower end of the tube (s), and air forced through the apparatus until it and the aræometer are perfectly clean and dry.

It is only possible to use the method as described above when the milk contains at least 2.07 per cent. fat; but Soxhlet has worked out a modification which can be used for milk poorer in fat, and also for separated milk and butter-milk. This consists in adding a solution of soap to the milk, for then the fat-containing ether separates more easily. The soap solution is prepared by dissolving $15 \mathrm{~g}$. of ordinary stearin candle in a mixture of 25 c.c. alcohol and 10 c.c. of the above- 
mentioned caustic potash solution (sp. gr. 1227). On warming, the stearin dissolves in a few minutes, and the solution is diluted to 100 c.c. with water. This solution sometimes becomes cloudy on keeping, but it is then only necessary to warm it to $30^{\circ} \mathrm{C}$. to bring it perfectly clear again. The addition of $20-25$ drops of the soap solution to 200 c.c. of separated milk is the only difference in the operation, the rest being carried out exactly as rescribed above for whole milk. If there should not be enough ether properly to carry out the experiment, then two bottles may be prepared from the same milk, and the ether from them united. For the determination of the specific gravity of the fat-containing ether, a specially constructed aræometer which gives the specific gravity between 0.743 and 0.721 must be used, and the percentage of fat calculated from Table II. in the Appendix.

For the examination of separated milk containing very little fat, as is often necessary now that machines are used, Soxhlet's method hardly gives as good results as those obtained from gravimetric analysis, but with the richer separated or skimmed milk, and above all with whole milk, the results are entirely satisfactory and reliable.

The apparatus required for Soxhlet's method can be obtained from Joh. Greiner, Munich, or any other firm supplying chemical apparatus.

\section{Wollny's Refractometer Method.}

Prof. Wollny, of Kiel, has worked out a new method for the estimation of the percentage of fat in milk which differs but little in principle from that of Soxhlet. Whereas the latter determined the specific gravity of the ethereal solution of fat got from the milk, Wollny determines the refractive index of such a solution by means of a specially constructed refractometer.

This method of course presupposes that the refractive index of milk fat is constant, which it is not, but the error which thereby arises is so small that it has no practical importance.

The refractometer, which is made by Carl Zeiss of Jena, resembles outwardly a microscope. A telescope provided with objective and eyepiece, and focussed for infinity, forms one of the principal parts of the refractometer. In the focus of the 
objective a scale, divided from $0-100$, is placed. The box containing the prisms is hollow and divided in half, and is affixed to the lower end of the telescope tube. It contains two rightangled prisms, the hypotenuse faces of which are placed parallel to one another with only a small space between them. One half of the box $(A)$, which contains one prism, is immovably fixed to the tube, but the other half $(B)$, in which the second

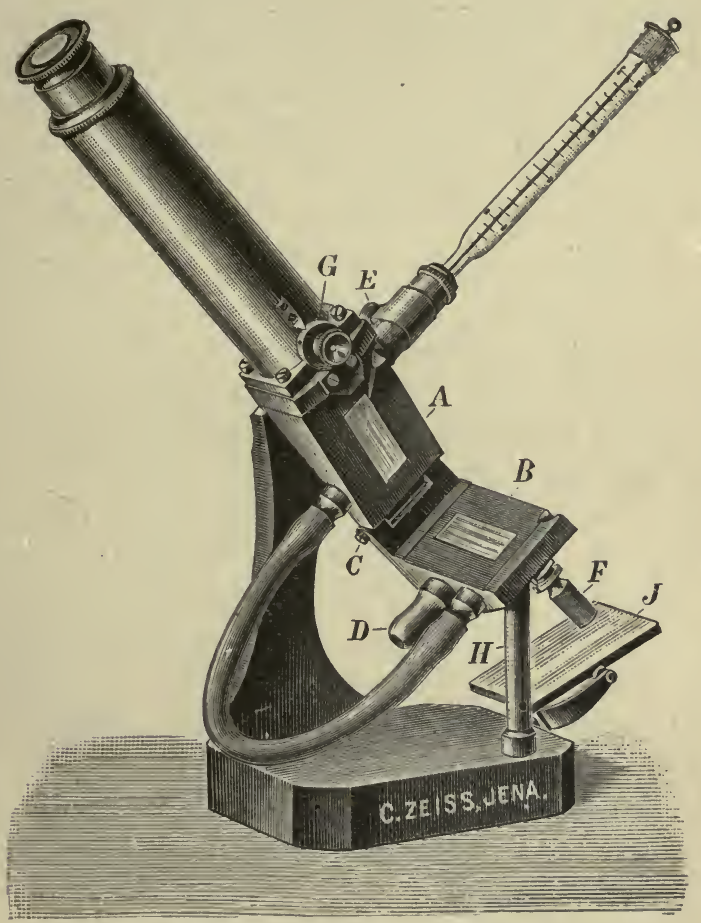

FIG. 13.-Wollny-Zeiss Milk Fat Refractometer.

prism is placed, can be moved on the hinge $(C)$ and opened by means of the fastening $\left(F^{\prime}\right)$. The two parts of the prism box are hollow, so that a stream of water can be passed round the prisms by means of the short piece of rubber tubing which connects them. On the foot of the stand there is fastened a plain mirror $(J)$ which sends light through the prisms. The apparatus is also provided with a specially constructed thermometer, which is placed in part $(A)$ of the prism box, for the 
reading of the refractive index must be done at a certain temperature, viz., $17 \cdot 5^{\circ} \mathrm{C}$. If a drop of the ether-fat solution is put into the narrow space between the prisms, it is seen on looking into the tube that a part of the field of view is bright, whilst the other part is quite dark. The dividing line between the dark and bright fields is very sharp. This is because a portion of the rays of light which enter the prisms is entirely refracted on passing through the ether-fat layer owing to the manner of arranging the prisms. Only some of the rays continue their way into the tube, so that part of the field of view is illuminated and part not. By means of the micrometer screw $(G)$, the dividing line between the bright and dark portions of the field is brought exactly on to a division of the scale. On reading off this division, a measure of the refractive index is obtained, and this varies according to the amount of fat dissolved in the ether. The greater the amount of fat, the greater the index of refraction. The thermometer which is used in the refractometer was specially designed by Wollny, and the point which corresponds to $17.5^{\circ} \mathrm{C}$. is taken as 0 . If the mercury stands at $0^{\circ}$, it is only necessary to read off the number on the scale of the refractometer to get the refractive index. When the thermometer is higher than $0^{\circ}$, the number of tenths of a division on the thermometer has to be added to the divisions of the refractometer scale, whilst for temperatures below $0^{\circ}$ a corresponding subtraction has to be made. If, for example, the refractometer scale reads $49 \cdot 1$ and the thermometer +0.3 , the correct reading is $49 \cdot 4$.

Pulfrich ${ }^{1}$ has constructed a special apparatus for supplying a stream of water of constant temperature, the arrangement of which is shown in Fig. 14.

For use with the refractometer, special glass tubes are made (see Fig. 15, on left) which have a mark at 30 c.c. In these tubes the milk is mixed with the caustic potash solution and ether.

The milk is measured by means of a self-filling pipette of 30 c.c., similar in construction to the one shown on the left of Fig. 15, and on the right of the same figure is shown a specially-constructed pipette for measuring the amount of ether required.

1 Zeitschrift für Instrumentenkunde, Jan, 1898. 


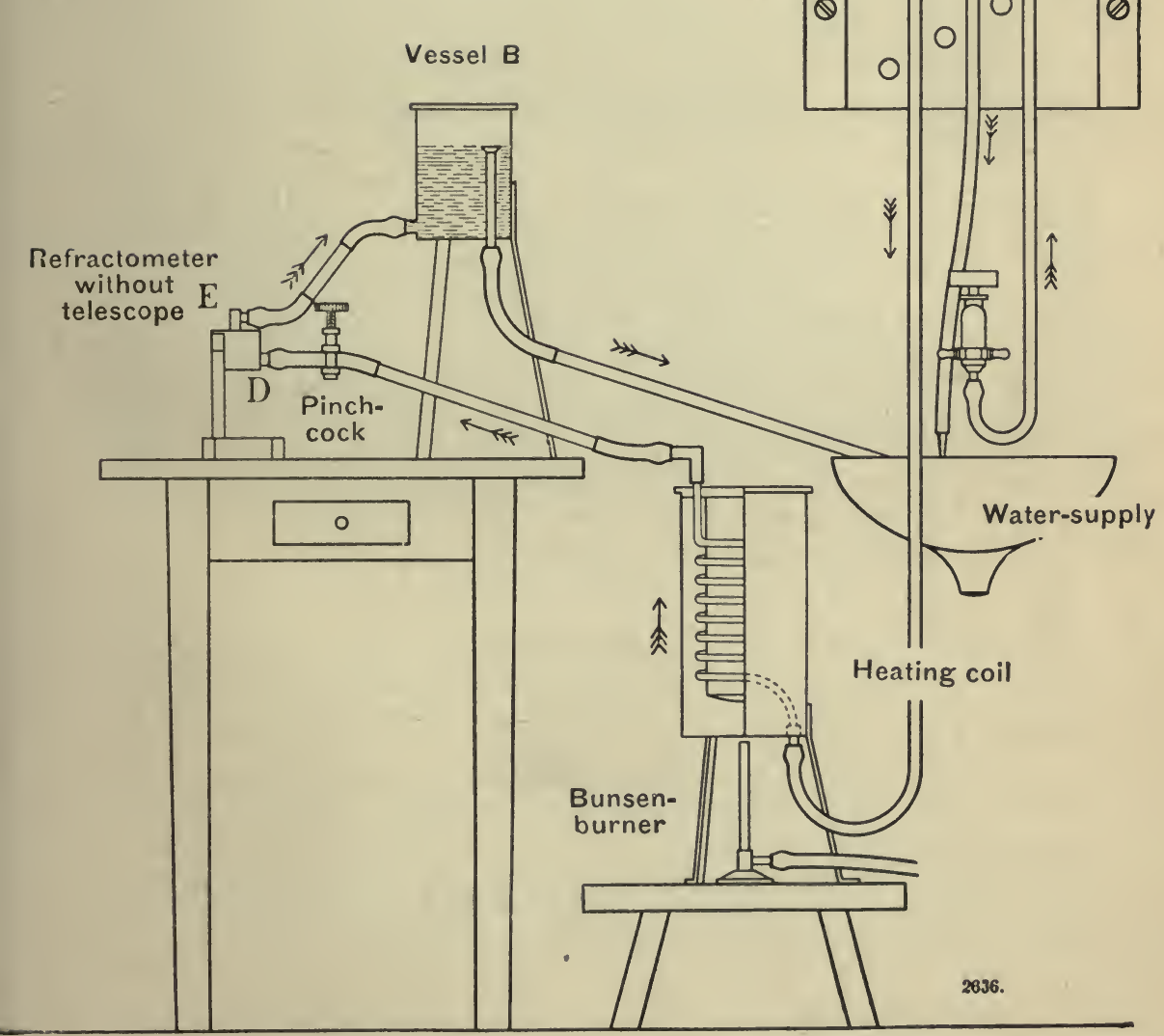

FIG. 14.-Apparatus for the Supply of Water at Constant:-Temperature. 
The author, though, has found that it is more satisfactory to measure the ether and also the caustic potash solution from a burette.

Naumann ${ }^{1}$ has carefully worked out the details of this method, and the following description is based upon his results. The necessary reagents are:

Caustic Potash Solution.--Eight hundred g. stick caustic potash are dissolved in a moderate quantity of water, and, after cooling, $600 \mathrm{~g}$. glycerine and $200 \mathrm{~g}$. copper hydrate (to be obtained from Th. Schuchardt, Görlitz) are added, and the mixture made up to 3 litres. After three or four days, during which time it should be shaken each day, the solution is ready for use. The addition of the copper makes the dividing line between the light and dark field more prominent.

Ether. - This should be saturated with water, which can be done by shaking the ether four times with distilled water, fresh water being added each time. The ether must have a temperature of $17.5^{\circ} \mathrm{C}$. when it is used.

Acetic Acid.-Ordinary glacial.

After the milk has been thoroughly mixed, 30 c.c. should be drawn out by means of the self-filling pipette, which is done by immersing the pipette completely, when a valve opens and the milk fills the reservoir. When the pipette is raised again the valve closes. The milk which adheres to the outside of the pipette is carefully wiped off, and by raising the valve the milk can flow into one of the tubes shown in Fig. 15, which it should fill exactly to the mark. Then three or four drops of acetic acid are added from a dropping bottle, and the tube shaken for one or two minutes. This can be done very satisfactorily with the help of the shaking machine made for the purpose by the firm of Franz Hugershoff, of Leipsic, ${ }^{2}$ the motive power being either a small water or electric motor (see Fig. 15).

When the samples are taken out of the shaking machine they must be brought to a temperature of $17.5^{\circ} \mathrm{C}$., and then 3 c.c. of caustic potash solution added from a burette. Then the tubes are shaken again, this time for ten minutes, after which they receive 6 c.c. of water-saturated ether, also from, a burette, and are shaken once more in the machine for fifteen minutes. In order to get the layer of ether to rise to the

1 Naumann. "Über die Untersuchung der Milch auf Fettgebalt mit dem von der Firma Carl Zeiss, Jena hergestellten Wollnyschen Milchfett refraktometer," Milch-Zeitung, 1900. No. 4 to 6. Reprint from M. Heinsius Nachfolger, Leipsic.

2 Carolinenstrasse, No. 13. The pipettes, tubes, \&c., which are used in the above method, as well as all other apparatus required in the investigation of milk and dairy products, can be obtained from this firm. 
top, the sample tubes are centrifuged in a suitable centrifuge (e.g. Gerber's) for three minutes, and a clear ethereal solution is obtained. Finally, the tubes are placed in a water-bath having a temperature of $17.5^{\circ} \mathrm{C}$.

Before beginning the determination by the refractometer, that instrument must be adjusted. This is done by putting a drop

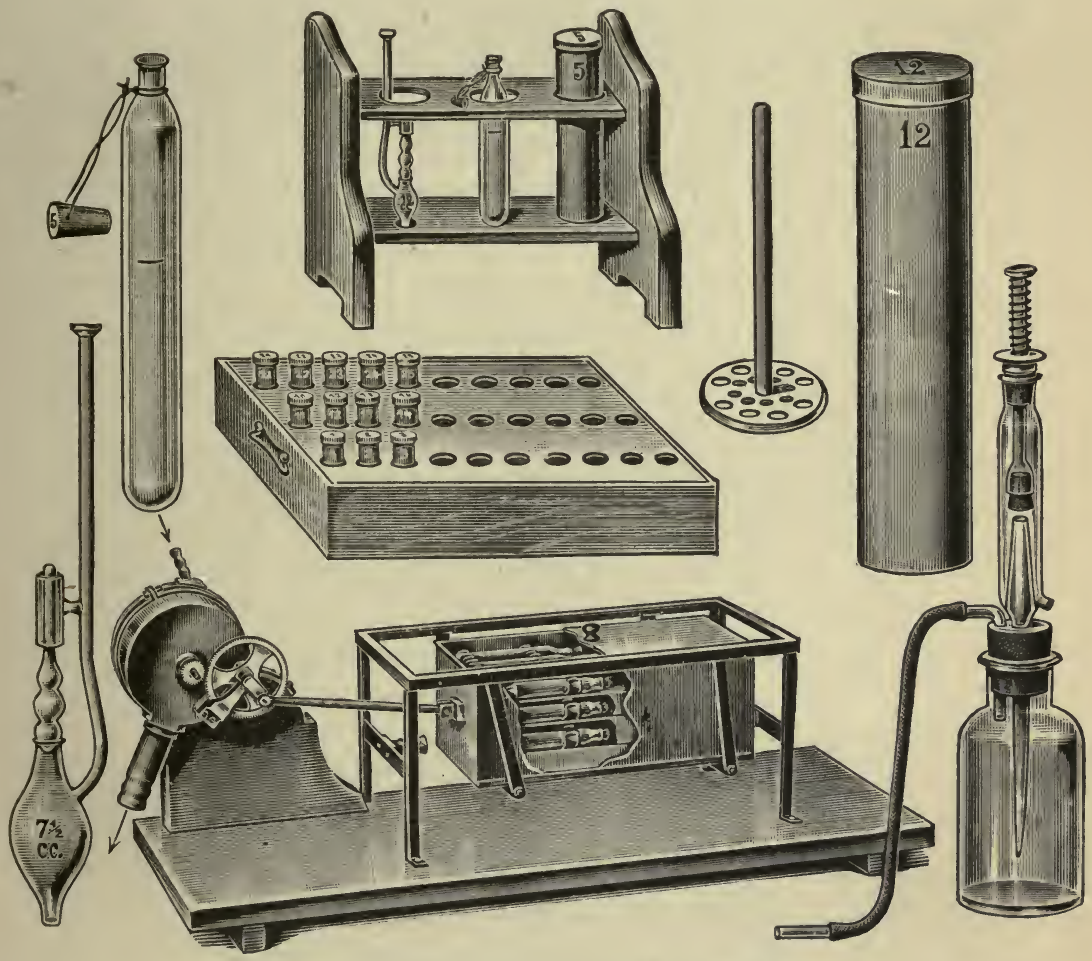

FIG. 15.-Apparatus for use in Wollny's Method.

of pure distilled water (temp. $17 \cdot 5^{\circ} \mathrm{C}$.) between the prisms, the water being introduced through the narrow canal which leads from the outside to the space between the faces of the prisms. If the instrument is correct and the temperature $17.5^{\circ} \mathrm{C}$., the boundary line between the light and dark field should fall upon the zero of the scale. After taking this reading, the lower prism is thrown back, and the surface of each prism carefully dried with a fine linen cloth. In a similar way a test is made 
with the water-saturated ether after the lower prism has been returned to its original position. The refractometer scale ought now to read 20.6 if the thermometer is at $17.5^{\circ} \mathrm{C}$. When taking these readings, the dividing line must be brought exactly on to the nearest division of the refractometer scale with the aid of the micrometer screw $(G)$ (see Fig. 13). To the number of whole divisions on the scale there must now be added the number of tenths of divisions on the scale of the micrometer screw. If the conditions have been found to be correct for the ether, then the determination of the index of refraction of the fat-containing ether can be made. After carefully cleaning and drying the opposed faces of the prisms, the prism box is closed and one of the sample tubes taken from the water-bath, where it has been kept at $17.5^{\circ} \mathrm{C}$. The cork is then removed, and a small glass tube some $10 \mathrm{~cm}$. long, open at both ends and with an internal diameter of $3-4 \mathrm{~mm}$., is dipped into the layer of ether. A few drops enter the tube in this way, the upper end of which is then closed with the finger and the tube withdrawn. By applying the lower end of this narrow tube to the mouth of the canal leading into the space between the prisms and removing the finger, the ether flows down, and the reading can be made immediately afterwards. If, for example, the scale reads 49 and that on the micrometer screw $0 \cdot 7$, then the total would be $49 \cdot 7$ divisions; and suppose the thermometer to read- $0 \cdot 2$, then the real number would be $49 \cdot 5$.

On referring to Naumann's tables (Table III in the Appendix), the percentage amount of fat corresponding to the above number can be seen; it is 3.44 per cent. ${ }^{1}$

The refractometer is adjusted for a yellow sodium flame, but it can be used without error for ordinary daylight or for lamplight. In the table below are given the indices of refraction corresponding to the divisions of the refractometer scale. The refractometer can also be used for determining the refractive index of other liquids, provided the refractive index lies between 1.33 and 1.42 (. g. milk serum).

1 On the proposal of M. Ripper, Zeiss has so arranged the scale of his refractometer that Naumann's tables are put alongside the ordinary scale, and this admits of the percentage of fat being read directly. The dividing line of the total reflexion crosses both scales. Corrections for temperature must naturally be made as before. 
Indices of Refraction Corresponding to the Divisions on the Refractometer Scale. ${ }^{1}$

\begin{tabular}{|c|c|c|c|c|c|c|c|c|}
\hline Division. & $n_{\mathrm{D}}$ & $\Delta_{n}$ & Division. & $n_{\mathrm{D}}$ & $\Delta_{n}$. & Division. & $n_{\mathrm{D}}$ & $\Delta_{n}$. \\
\hline-5 & $1 \cdot 3279$ & $10 \cdot 6$ & 35 & $1 \cdot 3683$ & $9 \cdot 4$ & 75 & $1 \cdot 4033$ & $7 \cdot 9$ \\
\hline 0 & $1: 3332$ & 10.5 & 40 & $1 \cdot 3730$ & $9 \cdot 2$ & 80 & 1.4072 & $7 \cdot 7$ \\
\hline 5 & $1 \cdot 3385$ & $10 \cdot 3$ & 45 & $1 \cdot 3776$ & $9 \cdot 0$ & 85 & 1.4111 & $7 \cdot 5$ \\
\hline 10 & $1 \cdot 3436$ & $10 \cdot 2$ & 50 & $1 \cdot 3821$ & $8 \cdot 8$ & 90 & 1.4148 & $7 \cdot 3$ \\
\hline 15 & $1 \cdot 3487$ & 10.0 & 55 & $1 \cdot 3866$ & $8 \cdot 6$ & 95 & $1 \cdot 4185$ & $7 \cdot 1$ \\
\hline 20) & $1 \cdot 3537$ & $9 \cdot 9$ & 60 & $1 \cdot 3909$ & 8.5 & 100 & 1.4220 & $7 \cdot 0$ \\
\hline 25 & $1 \cdot 3587$ & $9 \cdot 7$ & 65 & $1 \cdot 3951$ & $8 \cdot 3$ & 105 & 1.4255 & - \\
\hline 30 & $1 \cdot 3636$ & $9 \cdot 6$ & 70 & $1 \cdot 3993$ & $8 \cdot 1$ & - & - & - \\
\hline
\end{tabular}

Small differences in the composition of the caustic potash solution can have a very considerable effect upon the results got by the refractometer method. It is therefore advisable after making up a new supply of this solution to make several parallel tests with other reliable methods, e.g. Röse-Gottlieb's (see below), and to correct the refractometer determinations if differences are found.

If the test is carried, out exactly as described above, the refractometer gives very accurate results, but the method is so sensitive that the least departure from these rules influences the results. One great advantage, however, of this method is that where a large number of tests have to be made, it is possible, with the refractometer, to do 150 in an hour without effort.

With separated milk very good results are also obtained, as the author has shown, ${ }^{2}$ and he is also of the opinion that slight errors of manipulation do not have such a powerful influence as with whole milk.

\section{Extraction Methods.}

These methods are based upon the drying of a certain quantity of milk on some porous material, and then extracting the fat by means of ether or petroleum ether. On evaporation of the

1 The columns $\Delta_{n}$ give the changes in the refractive index-expressed as units of the fourth decimal-for each division on the scale.

${ }^{2}$ Revre Gén. du Lait, II, p. 145. 
ether, the fat can be dried and weighed. They are the oldest of all methods for the estimation of fat in milk, and numerous modifications of the actual operation have been proposed and used.

Originally, ignited sand, powdered pumice stone, gypsum, asbestos, or powdered glass were used, but now either paper (Adams) or granular kaolin (Nilson) are most usual, and only these will be described here.

\section{(a) Adams's Paper Coil Method.}

Adams's ${ }^{1}$ method consists of drying a weighed quantity of milk upon a strip of fat-free unsized paper, which, when dry, is rolled into a coil, and extracted with ether or petroleum ether in a suitable apparatus. The firm of Schleicher and Schiill, Diiren, prepare special strips of paper for this purpose, they measure 55 or $56 \mathrm{~cm}$. in length and $6 \mathrm{~cm}$. in width. The method as now practised is as follows: To weigh out the milk a small glass wash-bottle, weighing abont $30 \mathrm{~g}$., is used; it is about $8.5 \mathrm{~cm}$. high, diameter some $3.0 \mathrm{~cm}$., and a mark is made for each 5 c.c. contents. The jet is drawn out to a very fine point. The milk which is to be examined is then thoroughly mixed, poured into the clean dry bottle, and the whole weighed. After this, a clean piece of rubber tubing is attached to the mouth-piece of the bottle, and abont 10 c.c. of well-mixed milk are blown on to the strip of paper, ${ }^{2}$ which has been suspended horizontally.

After the milk has been brought on to the paper, the bottle is again weighed, and the difference shows the amount of milk used. The strip of paper is allowed to dry in the air, then rolled together, fastened by means of a fine platinum wire, and dried in the water-oven for two hours at $100^{\circ} \mathrm{C}$. It is best to suspend the coil from a loop of platinum to keep it out of contact with the walls or floor of the drying-oven.

After the drying is complete, the coil is put into a suitable

1 The Analyst, 1885 , p. 46.

2 Where extreme accuracy is needed, the specially prepared strips of paper shonld be extracted for several hours with ether before being used, for they are seldom absolutely free from materials soluble in ether (resins). At least, in such an extraction there is always a residne of a few milligrams, part of which, however, is fine paper fibre. With ordinary filter paper a previons extraction with ether is a necessity. 
extraction apparatus, on the bottom of which a layer of clean, previously extracted cotton wonl has been placed to keep back the paper fibres which would otherwise be carried over with the ether. Generally, Soxhlet's extraction apparatus (Fig. 16) is used, but others, e.g. those of Krayenbiihl, Storch, \&c., can equally well be employed.

The extraction apparatus is attached below to a carefullyweighed flask, and then enough pure anhydrous (distilled over sodium) ether is poured into the tube of the extraction apparatus to reach beyond the level of the syphon. The ether then flows into the flask below through the syphon. A suitable condenser must be attached to the upper part of the extraction tube. Formerly, a condenser, such as is seen in Fig. 16, or a spiral condenser was used, but latterly a more convenient shape which is not easily broken, for it is usually made of metal, has become general (see Fig. 17).

A condensing arrangement which deserves general recognition on account of its simplicity and efficiency has been designed by Prof. V. Storch of Copenhagen (see Fig. 18).

This condenser consists of a small cylinder of nickelled brass, $7 \mathrm{~cm}$. long and $2.7 \mathrm{~cm}$. in section. It is rounded underneath, and on the top is a curved disc, the edge of which projects $1 \mathrm{~cm}$. beyond the cylinder. Inside the condensing cylinder are two tubes for the water, one of which reaches almost to the bottom of the condenser, whilst the other only just passes through the disc. The condenser is hung loosely in the tube

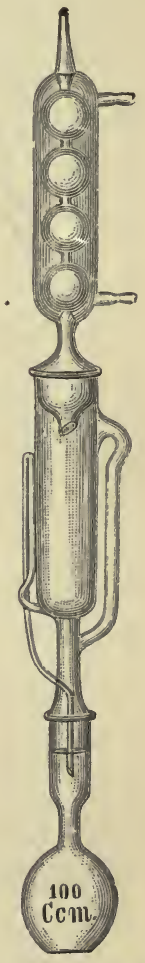

Frg. 16.-Soxhlet's Extraction Apparatus. of the extraction apparatus so that the projecting edges are closely joined to the open mouth of the tube. All the trouble of fixing the condenser to the extraction apparatus by means of a tightly fitting cork is thus avoided; the ether condenses on the lower part of the condenser, and there is no loss.

When the extraction apparatus has been fitted together, the 
flask is heated on the water-bath for about 8 hours, then it is detached after being allowed to cool, the ether evaporated or distilled off, and the flask, which now contains the fat, is dried for 2 hours in the water-oven at $100^{\circ} \mathrm{C}$. The flask is taken out at the end of this time, allowed to cool in a desiccator, and

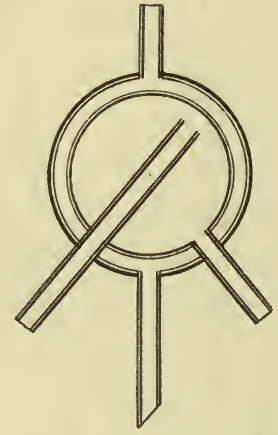

FIG. 17. - Soxhlet's Ball Condenser (cross section).

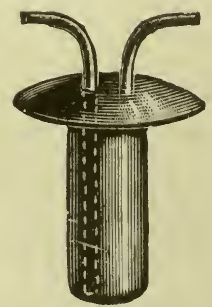

Fic: 18. - Storch Condenser for Use with the Extraction Apparatus.

then weighed. On deducting the weight of the empty flask, the weight of fat is obtained.

This method gives very good results with ordinary, that is whole, milk, and has long been regarded as the standard method for the estimation of the fat in milk.

\section{(b) Nilson's Kaolin Method.}

Professor L. F. Nilson of Stockholm ${ }^{1}$ introduced in 1891 the use of granular ignited kaolin as a substance on which to dry the milk. The grains of kaolin must be $0.5-1.5 \mathrm{~mm}$. in size, and $25-30 \mathrm{~g}$. of this material will go into one of the fatfree extraction cartridges made by Schleicher and Schüll. (Latterly aluminium cartridges have come into use for this purpose.) About $10 \mathrm{~g}$. of milk are weighed from a small beaker or flask in the usual way, the milk being poured over the kaolin in the cartridge. The kaolin absorbs all the milk, so that the walls of the cartridge are not wet. The cartridge with its contents is then dried for 2 hours in the water-oven, and

1 Kungl. Landtbruksakademiens Handl. och Tidskrift, 1891; Chem. Zeitung, 1891, No. 37. 
afterwards placed on a layer of extracted cotton wool in the Soxhlet apparatus. Ether-extracted cotton wool is also used to cover the kaolin in the cartridge. The extraction proceeds for 8 hours in the same manner as in Adams's method. With whole milk of normal composition very exact results are obtained with this kaolin method.

To determine the amount of fat in cream by either of the extraction methods, the procedure is similar to that with milk except that only $5 \mathrm{~g}$. of cream is taken. It is not possible either to use the small wash bottle for weighing out the cream in the Adams's method. 'This must be done in a small beaker, and the contents poured over the strip of paper. The materials formerly used to assist the drying, e.g. sand, powdered pumice, \&c., have been abandoned because they did not permit of the complete extraction of the fat. The methods described above, and in a greater degree those which were originally used, all have the defect that they give too low results for separated milk or for milk which has been subjected to energetic mechanical treatment, e.g. "homogenised" milk, milk heated by direct contact with a current of steam, or pasteurised milk which has been stirred too violently. This has been shown by the author ${ }^{1}$ to be due to the breaking up of the fat globules by the mechanical force, and the minute particles of fat which thereby arise become imbedded in the casein on drying, and are not reached by the ether during the extraction. The author has also proved that the further the division of the fat particles is carried, the lower are the results obtained. A longer period of extraction does not improve matters either. ${ }^{2}$ Numerous investigators have confirmed the author's explanation of the phenomenon. ${ }^{3}$

The methods about to be described do not suffer from the above-mentioned defect, for in them the fat comes in direct contact with the solvent, so the fine state of division of the fat has no influence upon the analysis.

1 Milch-Zeitung, 1903, No. 22. Molkerei-Zeitung (Berlin), 1903, No. 23 ; also Milch-Zeitung, 1903, No. 31 and No. 37.

2 Milch-Zeitung, 1904, No. 26.

3 L. F. Rosengren, Milch-Zeitung, 1904, No. 22 ; M. Siegfeld, MolkereiZeitung (Hildesheim), 1904, Nos. 39 and 40; M. Henseval, Revue Générale du Lait, Vol. III, p. 529 ; E. Holm, $56^{\text {te }}$ Beretning fra Kgl. Veterinaer-og Landbohöjskoles Laboratorium for landökonomiske Forsög, 1905. 


\section{Röse-Gottliel's Method.}

This method, which is now generally known as Gottlieb's method, is a modification by E. Gottlieb ${ }^{1}$ of the process devised by B. Röse ${ }^{2}$ for determining the percentage of fat in milk. The principle of the method is as follows: A certain quantity of milk is shaken with definite quantities of ammonia and alcohol whereby the casein dissolves. Then a known volume of ether and petroleum ether is added, and the whole well shaken in order to bring all the fat into solution. If the mixture is then allowed to stand in a measuring cylinder, a clear ethereal layer separates out, whilst below is an opaque liquid in which the other constituents of the milk, mostly dissolved in dilute alcohol, are contained. After the whole has stood for some time, the volume of ether is read, and an aliquot portion drawn off by means of a syphon into a tared flask, the ether-benzine evaporated off, and the fat which remains then weighed. The presence of benzine (petroleumether) is essential, for it prevents the solution of non-fatty substances which otherwise would dissolve in ether as the fat does.

In its present form the method is carried out as follows:

Ten c.c. of milk, which is almost exactly equivalent to $10.27 \mathrm{~g}$., are measured from a pipette into a tall, narrow cylinder, which is graduated to 0.5 c.c. and holds 100 c.c. Then 1 c.c. of ammonia (sp. gr. 0.96) is added from a burette, and also, with the help of a pipette, 10 c.c. of 95 per cent. alcohol. The cylinder is closed with a good cork, and then well shaken and frequently inverted. In this way the casein dissolves, and a thick homogeneous fluid is left, to which 25 c.c. of pure ether, not necessarily dry, are added from a burette or pipette. The cork is then replaced, and the shaking and reversing of the cylinder repeated to bring all the fat into solution. Finally, 25 c.c. of pure petroleum ether are added and the mixing once more thoroughly done. It should not be forgotten that if the cylinder is not well inverted each time the results will be too low. The petroleum ether must be completely volatile at

1 Die Landw. Versuchsstation, Vol. XL, p. 1.

2 Zeitschrift für angew. Chemie, 1888, p. 4. 
$60^{\circ} \mathrm{C}$., and on evaporation should not leave the least trace behind. After the above-mentioned final shaking the measuring cylinder and its contents are left undisturbed for 6 hours, so as to give the fat-containing ether time to separate completely. ${ }^{1}$

The ethereal solution of fat is now taken from the cylinder with the help of two tubes, which are fitted into a cork that exactly fits the mouth of the cylinder. One of the tubes which is bent at right angles only just passes through the cork, the other is longer and more bent, and has an internal diameter of $3-4 \mathrm{~mm}$. The cork with the tubes it contains is fitted to the mouth of the cylinder, and the longer tube, which is to serve as a syphon, is pushed down into the ether until it is exactly 1.5 c.c. above the dividing line of the two liquids. By blowing carefully into the shorter tube, the ether is forced into the longer one, and as this is arranged as a syphon the ether flows of its own accord. Instead of blowing into the tube, the rubber ball and tube used in Soxhlet's apparatus (p. 38) may be employed. The ether-benzine solution is collected in a tared flask of 100-150 c.c. contents with a fairly wide neck. By blowing carefully into the shorter tube the last few drops can be forced from the syphon, but care must be exercised, otherwise the liquid below the ether passes over as well. The 1.5 c.c. ether-fat solution which remain in the cylinder are exactly equivalent to the weight of $0.27 \mathrm{~g}$. milk. As the amount of milk taken for the test was $10 \cdot 27 \mathrm{~g}$., the fat, dissolved in ether-benzine, from $10 \mathrm{~g}$. of milk has been removed by the syphon.

After evaporating or distilling off the ether-benzine the fat which remains is dried at $100^{\circ} \mathrm{C}$. for several hours and weighed. The dry fat must be clear and fluid like oil, and not possess the least smell of benzine. When the weight of the fat which is left is multiplied by 10 , the percentage of fat in the milk is obtained without further calculation. With whole milk the Röse-Gottlieb and the extraction methods give results which agree very closely, but with separated milk and buttermilk the former always shows higher values. The differences, on an

1 M. Popp, Milch-Zeitung, 1904, No. 20, has shown that the result is not influenced if only one hour is allowed for the separation. He also states that the specific gravity of the ammonia does not need to be exactly 0.96 , but that it may vary between 0.91 and 0.96 without introducing an error. 
average, are 0.03 per cent. for separated milk, and for buttermilk more, sometimes as much as $0 \cdot 1$ per cent. The reason for this lies simply in the fact that in the latter cases the fat is in a very finely divided state, and so cannot be extracted completely after drying on some porous material.

L. F. Rosengren ${ }^{1}$ has shown that the fat obtained in the Röse-Gottlieb method always contains a little lecithin and gives an opalescence on redissolving in pure ether; the amount of lecithin is, however, so small as to be negligible.

For separated milk and buttermilk, Gottlieb's method is the best and most exact. M. Weibull, ${ }^{2}$ who from the beginning

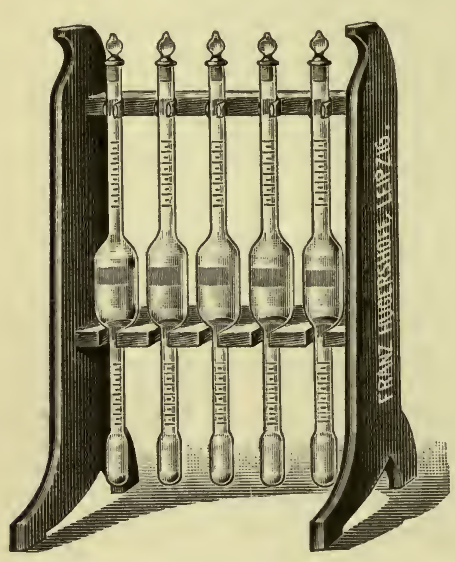

FIG. 19.-Farnsteiner's Tube for the Gottlieb Method. advocated its use, has shown that the results with separated milk and buttermilk, where the samples are analysed by different chemists, are the most reliable that can be obtained by any method.

When analysing cream, ${ }^{3} 3$ to $5 \mathrm{~g}$. are weighed from a small covered beaker into the Gottlieb cylinder, and the beaker again weighed. The cream in the cylinder is then diluted with water to exactly 10 c.c., and the determination made as for milk, save that as much as possible of the ethereal solution of fat should be drawn off, the cylinder again made up to its previous volume with ether-benzine (the distillate from a previous experiment serves very well), thoroughly shaken, and this second quantity syphoned off into the same flask. In this way the weight of fat which is left expresses all that was in the sample of cream.

K. Farnsteiner has designed a special form of tube for the Röse-Gottlieb method. The lower and middle parts of it are widened out, whilst that which lies between and carries the

1 Ref. p. 51.

2 Milch-Zeitung, 1898, p. 406 ; Chem. Zeitung, No. 63.

3 A. Hesse, Molkerei-Zeitung (Hildesheim), 1902, p. 49 ; M. Siegfeld and M. Popp, ibid., 1903, No. 13. 
graduation marks is narrow. This tube possesses the advantage that the volume of fat-containing ether which is drawn off here with a pipette, not a syphon, can be easily read.

Recently Röhrig has modified the original Gottlieb cylinder by affixing to the side a glass tap through which an aliquot part of the ethereal solution can be drawn off direct into a tared flask. 'The apparatus is very convenient to work with.

Another modification of the Gottlieb cylinder is in the form of a glass tube about $37 \mathrm{~cm}$. long, and internal diameter $2 \mathrm{~cm}$. The tube is rounded at the bottom so that it can be placed in a suitable support and has only two marks, one at 19 c.c. and the other at 70.5 c.c. As a rule the boundary between the cloudy and the clear layer lies at 17.5 c.c. (if the milk is not unusually rich in fat). If, therefore, in syphoning off the ether the lower end of the syphon is placed at the division 19 c.c., there remains behind exactly 1.5 c.c. On the other hand, the total volume of liquid in the tube is generally about 70.5 c.c. before any is drawn off.

For whole milk and for exact analyses of separated milk the completely graduated

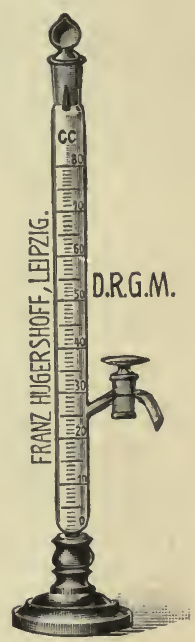

Fig. 20.-Röhrig's Tube for Gottlieb Method. cylinder must be used, but for ordinary determination with separated milk the above-described simpler and considerably cheaper tube can be used. In the analysis of separated milk the line between the two layers of liquid comes practically always to the same position in the tube.

\section{Liebermann-Székely's ${ }^{1}$ Method.}

In this method petroleum ether alone is used as a solvent for the fat.

After the specific gravity of the milk has been determined by Soxhlet's lactometer or the Westphal balance, 50 c.c. of milk at room temperature are brought into a graduated cylinder about

1 Zeitschrift. f. anal. Chemie, Vol. XXXII, 1893, p. 168. 
$25 \mathrm{~cm}$. high and $4.5 \mathrm{~cm}$. wide along with 5 c.c. of caustic potash (sp. gr. 1.27), and the cylinder corked and well shaken. Afterwards 50 c.c. of pure, light petroleum (b. p. about $60^{\circ}$ ) are added, and the cylinder again thoroughly shaken. An emulsion forms and to it is added 50 c.c. of alcohol (95 $8-96$ per cent.) and the shaking repeated.

At the end of about five minutes the petroleum ether has completely separated out. A final shaking-three or four times for periods of four minutes, the ether being allowed to separate after each time-is given, and 20 c.c. of the petroleum ether solution drawn off with a pipette into a tared flask. After the petroleum ether has evaporated, the fat is dried at $105^{\circ} \mathrm{C}$. and weighed. The relation which the whole of the petroleum ether bears to the 20 c.c. which were drawn off is then determined, and as the specific gravity of the milk is known, it is easy to calculate the percestage of fat which it contains.

This method also gives good and reliable results, and has the same advantages over the extraction methods as has that of Röse-Gottlieb. It is cheaper, though, than the latter, as petroleum ether alone is used, and not a mixture of this and ether; but, on the other hand, the evaporation of the petroleum ether takes longer than does the mixed solvent.

\section{Weibull's Desiccation Method for the Estimation of Fat in Cream.}

The method proposed by M. Weibull ${ }^{1}$ in 1896 for estimating the percentage of fat in cream is very easy to carry out, and yields results sufficiently accurate for most purposes. The difference between Weibull's method and the ordinary extraction method with ether (which gives very good results with cream) is seldom more than 0.5 per cent., and never more than 1 per cent., which for cream is accurate enough.

Weibull's method is based upon the principle that as cream consists of fat and milk serum, and the latter has a very constant composition, the composition of cream serum must also be fairly constant. As the total solids not fat of milk are

1 Landtbruksakad, Handl, o. Tidskrift, 1896, p. 370. Chem. Zeitung, 1907, I, p. 333. 
constant, or nearly so, whilst in milk or cream they diminish in proportion to the increase of fat, the following formula, based upon the relation between fat and total solids (both expressed in percentages of the cream), can be used:

$$
d=f+\frac{100-f}{100} \times \text { constant }
$$

in which $d=$ dry matter or total solids and $f=$ fat contents.

The size of the constant (per cent. total solids not fat) lies within narrow limits, and for cows of the Low Countries' breeds is 8.7 . The amount of total solids not fat in the milk of various breeds of cows is shown in the following table, which is due to J. König : ${ }^{1}$

\section{Per cent. total solids} not fat.

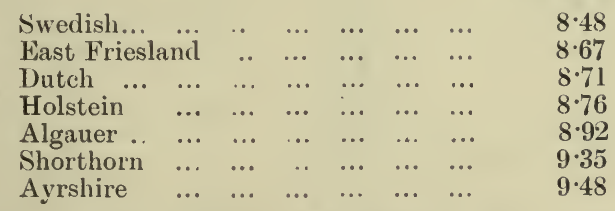

For cattle in the North of Germany the constant may be taken as 8.75 ; in other countries and for other breeds the constant would be different, as the above table shows.

By determining the total solids of the cream and placing this in the above formula, the fat contents is obtained. As previously mentioned, the change in the constant, due to the breed and country, would necessitate a determination of it for the particular district in which the test was being used.

The manipulation is very simple. Into a small flat basin, which contains about 20 c.c. of very finely powdered pumice stone, $6 \mathrm{~g}$. of the cream are weighed in the usual way from a beaker. After drying for $2 \frac{1}{2}$ hours in the water-oven at $100^{\circ} \mathrm{C}$., the dry matter is weighed. The percentage of fat can then be calculated with the help of the above formula, or more simply and conveniently by reference to the table drawn up by Weibull (Table IV in the Appendix), where the fat contents is shown directly from the weight of the total solids. This table is based upon a constant of 8.7 . Where sour cream has to be tested, the method with ammonia, described on p. 21, can be used in order to get the cream into a fluid state, after which the desiccation, \&c., can be carried out in the manner described above.

1 Die menschlichen Nahrungs- und Genussmittel, Berlin, 1899, Vol. II, 230. 


\section{B. Practical Methods.}

\section{The "Lactocrite" Method.}

This method, which was worked out by de Laval in 1886, is based upon the following considerations: A certain quantity of milk is treated with a liquid which possesses the power of

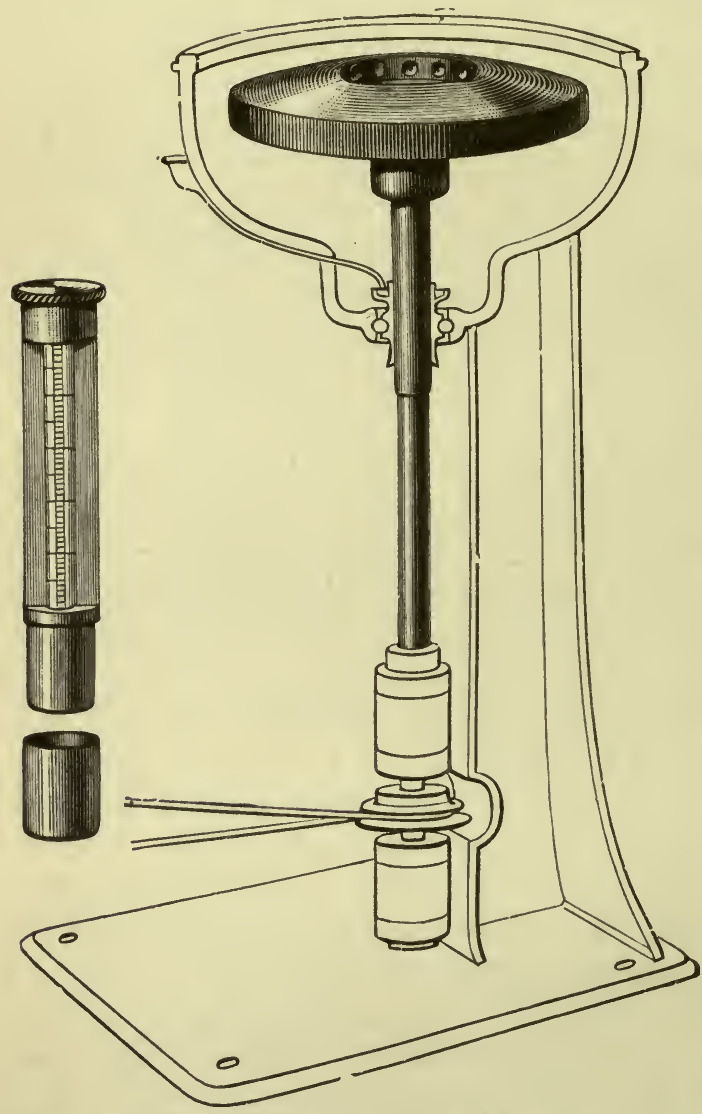

dissolving all the substances present in milk with the exception of the fat. When an aliquot part of this mixture is placed in a "tester" provided with a graduated capillary tube and whirled in a centrifuge, the fat separates. Originally a mixture of acetic acid with 5 per cent. concentrated sulphuric acid was used to dissolve the casein; afterwards ethylidenelactic acid with 5 per cent. sulphuric acid and

FIG. 21.-Tester, Disc, and Stand used in the Lactocrite Method.

10 per cent. hydrochloric acid were employed. ${ }^{1}$

The testing tubes are graduated empirically after very careful comparison with the gravimetric analysis.

1 M. Ekenberg, Die Laktokritmethode, dec. Iuaug. Diss., Königsberg, 1893. 
The apparatus required for the lactocrite method is made by the Aktiebolaget Separator of Stockholm.

It consists of the "testers," which are made of silvered or platinised metal, with graduated glass tubes; a disc which can be fitted to the stand of any Alfa-Laval separator, and has cavities bored into it to hold the testers (Fig. 21); a water bath (Fig. 22); a cover for the disc so that it can be heated by steam; a number of boiling tubes (Fig. 23).

To carry out the test 10 c.c. of milk at $15^{\circ} \mathrm{C}$. are measured into a boiling tube which already contains 10 c.c. of the acid mixture heated to $80^{\circ}$. After mixing, the boiling tube is heated in the waterbath at $100^{\circ} \mathrm{C}$. for 15 to 18 minutes with occasional shakings.

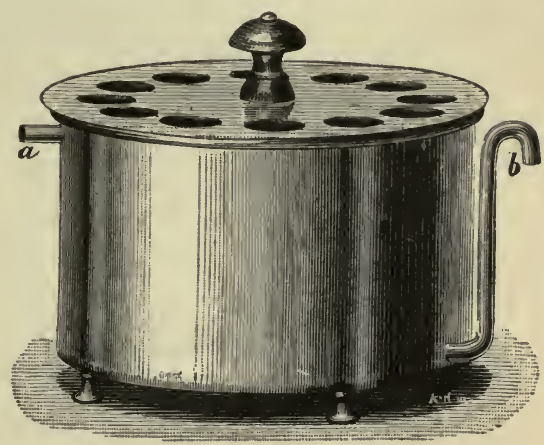

Fig. 22.-Water-bath for the Lactocrite Method.

As will be seen in

Fig. 23 the boiling tube is provided with a piece of glass tubing which acts as a reflux condenser. The boiling tubes are heated in the water-bath, shown in Fig. 22, which is arranged for steam, but can also be heated by a burner. When the heating is finished cold water is slowly led into the waterbath to cool down the boiling tubes. Finally, the holder containing the boiling tubes is placed in water at $60^{\circ}$, where it remains until the temperature shown on a thermometer placed in one of the boiling tubes also registers $60^{\circ}$.

In the meantime the testers have been placed in the disc and warmed to about $60^{\circ} \mathrm{C}$. by slowly revolving the disc on its support and letting steam into the cover which is provided for the purpose of heating up the disc.

After violently shaking one of the boiling tubes, part of the contents is poured into the small cup belonging to the tester, and the latter immediately pushed into the cup. The graduated glass tube fills with the liquid, and the excess spirts out through the opening in the upper part of the tester. The filling of the testers must therefore be done over a porcelain basin. This 
operation of filling the testers must be performed as rapidly as possible, otherwise the fat will rise to the surface, and unsatisfactory results will be obtained.

The testers, after being filled, are put into the disc, and if they do not fill all the places they should be so arranged that they lie opposite to one another. Should there be an unequal number of testers, the disc will not rotate evenly, so it is best

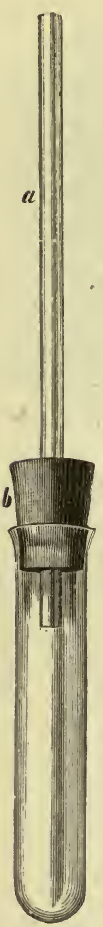

FIG. 23. - Boiling Tube for the Iactocrite Method. to make up an equal number with the help of a tester filled with water. When the testers are in position, water is poured into the bottom of the disc so as to bring about an opposing hydrostatic pressure, and the disc is at once set in motion, steam being blown in to bring the temperature to $60^{\circ} \mathrm{C}$. When the speed reaches $5,000-7,000$ revolutions per minute, this rate is maintained for 4 or 5 minutes.

When the testers are taken from the disc, the small hole in the upper part is closed with the finger, the tester held against the light, and the height of the column of fat read off upon the scale (see Fig. 24). Each division of this is equivalent to $0 \cdot 1$ per cent. fat, so that the percentage weight can be read off directly. At the open end of the fat column is a meniscus, and the reading is taken from the lowest point of this.

The testers are cleaned most easily by washing with weak soda lye and warm water, after which a dry cotton cord should be drawn through the capillary.

The lactocrite method gives results with whole milk which agree very closely with those got by the gravimetric form of analysis, as the investigations of L. F. Nilson ${ }^{1}$ show, and so can be used where accuracy is essential. Those methods which will now be described, and which depend upon the use of a centrifuge for the estimation of the fat in milk, are really only modifications of de Laval's original method. 


\section{Babcocl's Method.}

In 1890 Dr. S. M. Babcock ${ }^{1}$ recommended the following method for determining volumetrically the percentage of fat in milk. It is baser upon the treatment of a known quantity of milk with a definite volume of concentrated sulphuric acid (sp. gr. $1.82-1.83)$ in a glass test-bottle of special shape. This causes the casein to dissolve; and after the sample has been whirled in the centrifuge the test-bottle is filled almost full with boiling water and again centrifuged. When the test-bottle is taken

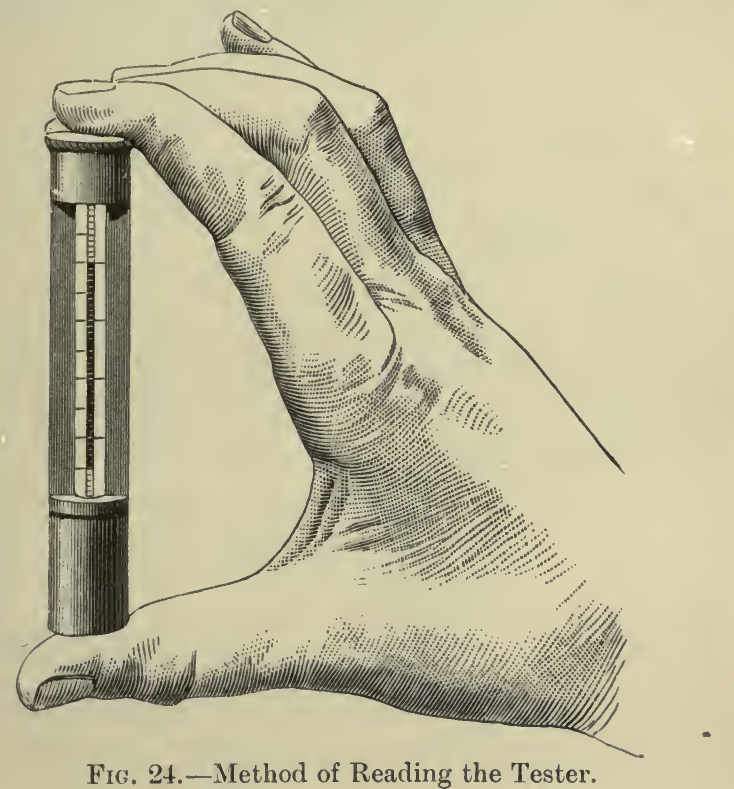

from the centrifuge, the height of the column of fat can be read off on the graduated neck. The apparatus is shown in Fig. 25, and a sketch of the test-bottle in Fig. 26.

The actual test is carried out as follows: The well-mixed milk is measured in the special $17 \cdot 6$ c.c. pipette, and then run into the test-bottle. With the help of a small glass cylinder $17 \cdot 5$ c.c. of sulphuric acid (ordinary commercial acid, sp. gr. $1 \cdot 82-1 \cdot 83$ ), are measured out and poured into the test-bottle, which is held obliquely so that the acid runs to the bottom and

1 Seventh Ann. Rep. of the Agr. Expt. Stat. of the University of Wisconsin, 1889-1890, p. 98. 
collects below the milk. When all the samples have been treated in this way, the contents of each bottle is mixed by giving the bottle a slow rotary motion, the neck being held in the hand. When all the casein, \&c, has dissolved, the testbottles are put into a special centrifuge, care being exercised, as in the "lactocrite" method, to get an even balance by placing the bottles opposite to each other if there are not enough to fill the centrifuge. If there are an unequal number of test-bottles, one should be filled with water to make up the required number. This rule applies to all methods where the centrifuge is used, so need not be repeated each time.
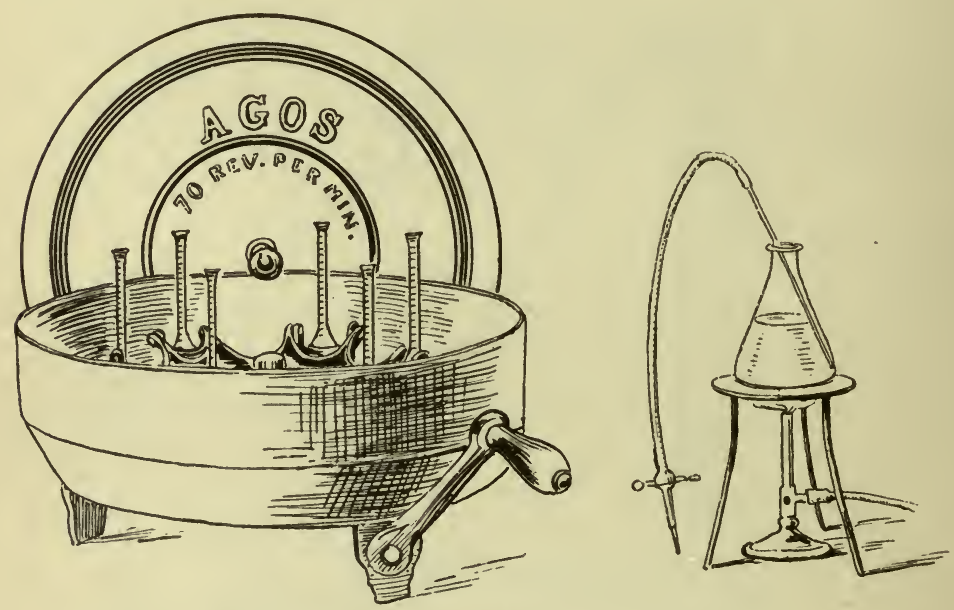

Fics. 25a, 25b.-Babcock's Milk-testing Apparatus.

When the test-bottles are in the centrifuge, boiling water is poured into the latter until the level of the water is up to the height of the vertically standing test-bottles. It is better, however, to use steam for heating the centrifuge. The cover is then put on the centrifuge, and the latter whirled for 6 or 7 minutes at a rate of $700-1,200$ revolutions per minute. There are various makes of centrifuges to be obtained, either for turning by hand or power.

After whirling in the centrifuge the test-bottles are filled with boiling water, the arrangement shown in Fig. $25 b$ being handy for the purpose. The water is boiled in the conical 
flask and then drawn off, by means of a syphon provided with a pinch-cock, into the test-bottles. In this way the test-bottles are filled to the 7 th or 8 th division of the scale.

In the new centrifuges which are heated by steam there is a very practical arrangement for filling the bottles with hot water. A reservoir of water placed above the centrifuge is heated by the steam which is used for warming up the centrifuge, and from it, by means of a rubber tube, the testbottles can be filled without being taken out of the centrifuge. The test-bottles are, of course, in a vertical position when the centrifuge is not in motion.

After adding the water, the test-bottles are centrifuged for one minute and then read. The fat ought to be a clear, welldefined column in the graduated tube of the test-bottle; if it is cloudy, then the bottles must be slowly whirled again, the centrifuge being heated. Generally the fat becomes fairly clear after this.

Both the upper and lower limits of the column of fat are somewhat uneven. Each division is equal to 0.2 per cent. fat. For analysing cream by Babcock's method, specially constructed bottles are used.

Ohlsson has also constructed a test-bottle of special shape for determining the fat in separated milk (see Fig. 28).

As the column of fat is very short with separated milk, the graduated part of the test-bottle is made very narrow, so that a longer and more easily-read column is obtained. The milk, sulphuric acid, and water are not filled in through the ordinary neck of the bottle, but through a special side tube. Each division on the scale represents 0.05 per cent. of fat.

After the Babcock bottles have been emptied they are washed in soda lye, rinsed in warm water, and aliowed to drain upside down. 
The results obtained by the Babcock test with whole milk are very satisfactory, and compare closely with those got by the gravimetric methods.

For exact determination of fat in separated milk this Babcock method is not to be recommended, even with Ohlsson's improved bottles (which are, unfortunately, easily broken). It can be used for testing milk from a separator to see if it is working properly within certain limits, but a more exact determination should not be attempted, as this method gives a lower result $(0.06-0.08$ per cent.) than does the Röse-Gottlieb method.

The Babcock test is used chiefly

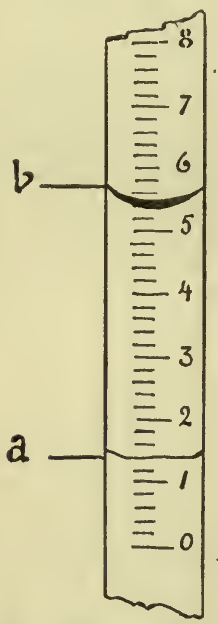

FIa. 27.- Reading the Column of Fat.

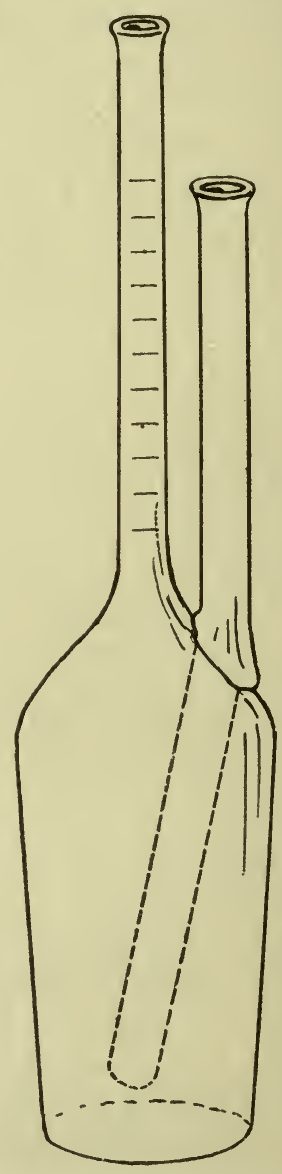

Fig: 28.-Ohlsson's Double-necked Bottle for Determining the Fat in Separated Milk.

in America and Australia; in fact, in North America it is almost exclusively the sole "practical" method employed. 


\section{Lindström's Butyrometer Method.}

In 1889 I. Lindström ${ }^{1}$ proposed a simple and efficient centrifugal method for estimating the fat in milk, which has been improved by the Aktiebolaget Separator of Stockholm. Originally the same mixture of acids was used as in the lactocrite method, but this was soon replaced by concentrated sulphuric acid as a solvent for the casein and the undissolved phosphate in the milk. The principle of this method in its present form is therefore the same as in the Babcock test.

A noticeable difference between Lindström's butyrometer method and the other centrifugal methods is, that in the former the column of fat which separates in the graduated part of the tube is allowed to cool and become solid; this has the advantage that the samples need not be read immediately after being taken from the centrifuge, but may be kept for any length of time.

Lindström's butyrometer is made by the Aktiebolaget Separator of Stockholm, in two sizes. The large one, which holds eighty test-bottles, can be driven by power in the ordinary Alfa-Laval separator stand, or by hand in the special support belonging to the Baby butyrometer. The smalier size is also turned by hand; it holds twenty test-bottles, and is known as the Kolibri butyrometer. In Fig. 29 the large butyrometer and the apparatus belonging to it are shown, whilst in Fig. 30 is a sketch of the small butyrometer.

The following parts belong to the butyrometer:

(1) The test-bottles (Fig. 29, 1), consisting of a narrow graduated tube, which is joined on to a wider one. The latter has a small opening on the upper rounded portion, and a distinct mark round the middle. A number is etched on the upper part of the test-bottle.

(2) Pipettes to measure the milk (Fig. 29). In ordinary cases (6) is used, whilst (5) is reserved for milk which has been treated with preservatives, for it prevents the milk from entering the mouth when it is drawn into the pipette.

(3) A wooden stand for the test-bottles (Fig. 29, 10).

(4) Glass measuring cylinder for sulphuric acid (Fig. 29, 4).

(5) Crude sulphuric acid of $1.80 \mathrm{sp}$. gr.

1 Milch.Zeitung, 1892, p. 496, and 1893, pp. 85, 341. 
The experiment is made in the following manner: The testbottle is held in the left hand so that the first finger closes the graduated tube, and the thumb the small opening on the side of the test-bottle; thus the lower part of the latter points upwards. With the help of the small glass cylinder the bottle is filled with sulphuric acid to the mark. Then the milk is

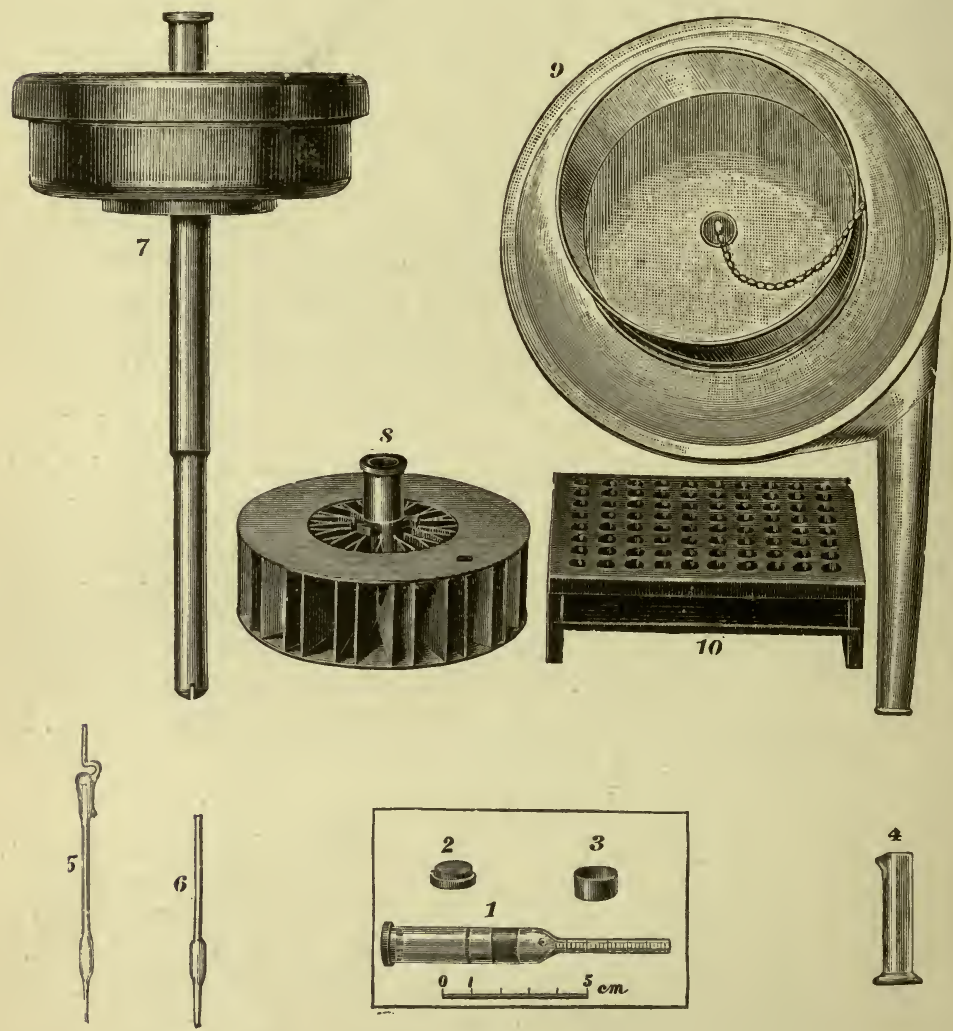

Frg. 29.-The Large Butyrometer and Component Parts.

well mixed by pouring it from one vessel to another, and the pipette (which holds 1.54 c.c.) filled by sucking until milk enters the mouth; afterwards the pipette is closed with the tip of the tongue. The contents of the pipette are then easily transferred to the test-bottle, the milk being blown in so that it mixes with the sulphuric acid. A rubber cork is used 
to close the test-bottle, which must be well shaken. After the test-bottle has been turned, with the rubber stopper underneath, so that a small air-space is formed at the side opening, the first finger is removed. 'This must not be done before, otherwise the liquid in the graduated tube will spirt out. The test-bottle
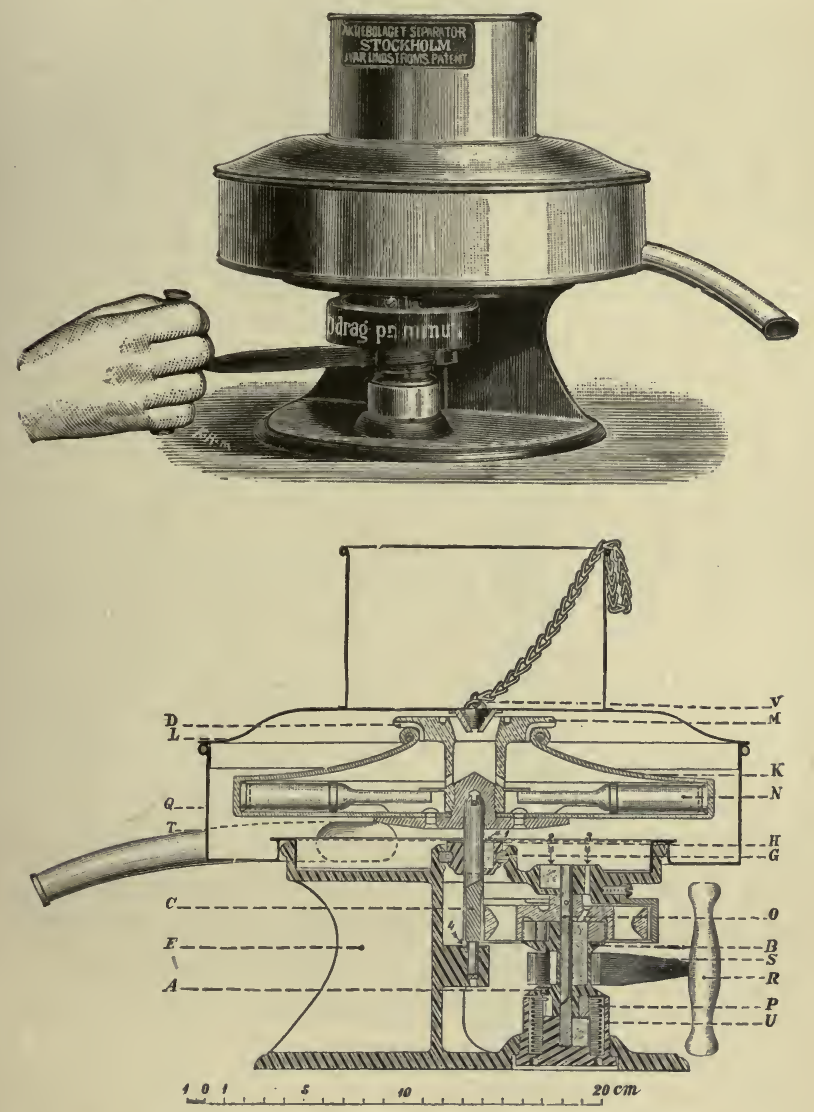

Fic. 30.-The Kolibri Butyrometer.

is then placed in the stand and another taken up and filled. If the large butyrometer, the one intended for eighty tests, is to be used, the test-bottles must be so arranged that the small opening is always pointing upwards, so that none of the contents flows out. Five test-bottles can be placed in each compartment, and they must be held together in a horizontal position by a 
rubber band. With the Kolibri butyrometer the rubber band is not necessary, for the test-bottles are placed singly in the compartments (see Fig. 30 b).

After the test-bottles have been put into place the lid is screwed on, and the disc placed in the stand, after which the water-tank is put on, care being taken that the valve is closed. Warm water of at least $65^{\circ} \mathrm{C}$. is poured into the tank, and the disc rotated with increasing speed. When the rate of speed is fairly high, the valve on the water-tank is opened and warm water allowed to flow into the disc. The latter soon fills, and water begins to flow out of the tube of the water-tank. The speed is then raised to that usual for an Alfa-Laval separator, namely, 5,600 revolutions per minute, and this is continued for at least five minutes. During this process warm water enters by the small opening into the interior of the test-bottle and drives the separated fat into the graduated part.

At the end of the above-mentioned time ice-cold water is poured into the water-tank, the disc still revolving, but with diminishing speed. When the temperature of the water which flows away is not higher than $12^{\circ} \mathrm{C}$, the centrifuge may be stopped, and the test-bottles taken out and put into the wooden stand. The fat is then in a pale, homogeneous condition in the graduated tube, and the column is clearly defined. Each division of the scale of the test-bottle is equivalent to 0.2. per cent. fat, but it is quite easy to read to 0.05 per cent. The number which is read off gives the percentage amount of fat in the milk.

With cream very satisfactory results can be obtained in the following manner: 14 c.c. water of $30^{\circ}-40^{\circ} \mathrm{C}$. temperature are brought into a small, dry beaker by means of a pipette. The cream is measured out in the usual small pipette, the outside of which must afterwards be dried, and then blown into the water. By repeatedly drawing up the water and allowing it to flow out again, the inside of the pipette is freed from particles of cream. With this mixture of cream and water the test is made in the butyrometer in the ordinary way. The number found on the scale is multiplied by ten, and this gives the percentage amount of fat found in the cream. According to investigations by the author, ${ }^{1}$ this method gives very good

\footnotetext{
${ }^{1}$ Nordisk Mejeri-Tidning, 1899, No. 34.
} 
results with cream. With separated milk, Lindström's butyrometer gives altogether unreliable figures on account of the small quantity of fat which is got.

The test-bottles are cleaned in the same manner as in the Babcock test. Lindström's butyrometer method is used almost without exception in Sweden, Norway, and Finland fur a practical determination of the amount of fat in milk. The accuracy is about the same as with the Babcock or Gerber methods, and the results with whole milk generally agree to within $0 \cdot 1$ per cent. of those found by gravimetric analysis.

\section{Gerber's Method.}

In $1892^{1}$ Dr. N. Gerber, of Zurich, published details of the method which he had worked out for the determination of fat. This method, however, only assumed its present form in 1895, and at the same time it was expanded into a "universal method for the estimation of fat," to serve for the analysis of milk and other dairy products. To dissolve the casein Gerber also uses concentrated sulphuric acid, but the method differs from others in the addition of amyl alcohol, which Leffmann
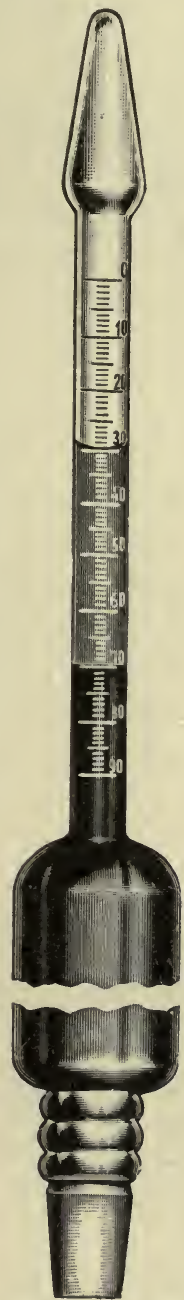

Fıc. 31.-Gerber's Test-Bottles or Acido-Butyrometers. and Beam ${ }^{2}$ had shown to facilitate the separation of the fat in a pure, clear condition.

1 Milch-Zeitung, 1892 , p. 891 ; 1893, pp. 363 and 656 ; 1895, p. 169.

- Analyst, Vol. XVII, 1892, p. 83. 
The following apparatus and reagents are required for Gerber's "acidobutyrometric" method:

(1) Test-bottles of glass. These have a wide main portion, which is joined to a graduated tube ending in a conical expansion (see Fig. 31).

(2) Pipettes to measure out milk, sulphuric acid, and amyl alcohol. The pipette for the milk holds $11 \mathrm{~cm}$. (see Fig. 32),

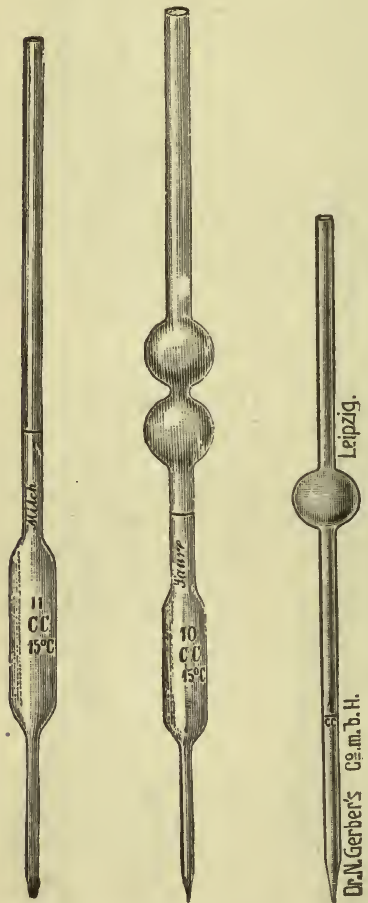

Fit. 32.-Pipettes for Milk, Acid, Amyl Alcohol.

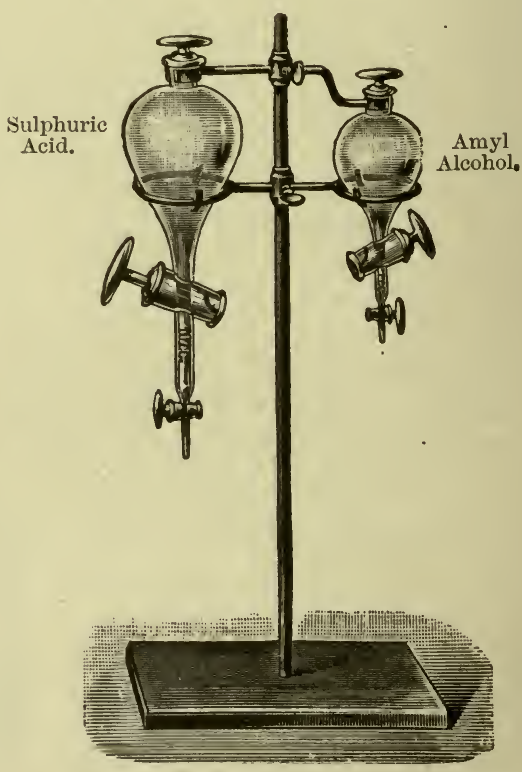

Fig. 33.-Automatic Measurer of Sulphuric Acid and Amyl Alcohol.

and the one for the acid $10 \mathrm{~cm}$. This latter is provided with two bulbs, which prevent the entrance of acid into the mouth when the pipette is being used. For amyl alcohol the pipette holds only 1 c.c. Various forms of apparatus designed to automatically measure the sulphuric acid, and also the amyl alcohol, have been invented, but a description of them is not necessary here. Two of the most useful are shown in Figs. 33 
and 34, the way in which they work being easily seen from the sketches.

(3) Wooden stand to hold the bottles.

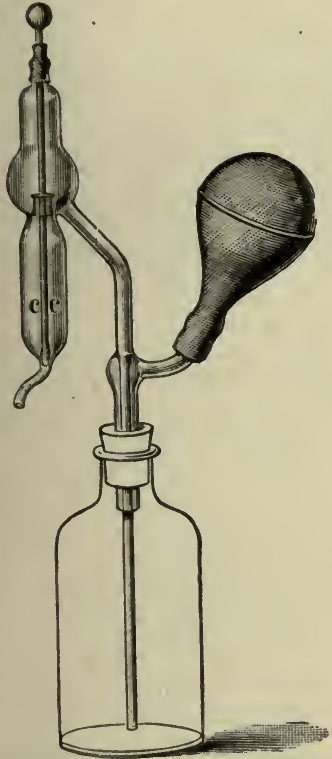

FIG. 34.-Automatic Mea surer of Sulphuric Acid.

(4) Water-bath of tin, with lamp; for heating the test-bottles (see Fig. $35)$.

(5) Centrifuge for whirling the samples. Such centrifuges are made in several forms, and are driven by hand, steam, or electricity, and some have an arrangement for heating whilst in motion (see Fig. 36).

(6) Commercially-pure sulphuric acid of a specific gravity of 1.825 at $15^{\circ} \mathrm{C}$.

(7) Amyl alcohol. The properties of this exercise a considerable influence upon the result of the analysis. It must be chemically pure, the specific gravity 0.815 at $15^{\circ} \mathrm{C}$., and the boiling point $128^{\circ}-130^{\circ} \mathrm{C} .^{1}$

The determination of the fat is made in the following manner:

The test-bottles are put into the

stand with the mouths uppermost; then, with the pipette designed for the purpose or with an automatic measurer, 10 c.c. of sulphuric acid are filled into the test-bottle, care being taken not to allow any to come in contact with the neck. The few drops remaining in the tip of the pipette should not be blown out. Then 11 c.c. of milk are measured with the pro-

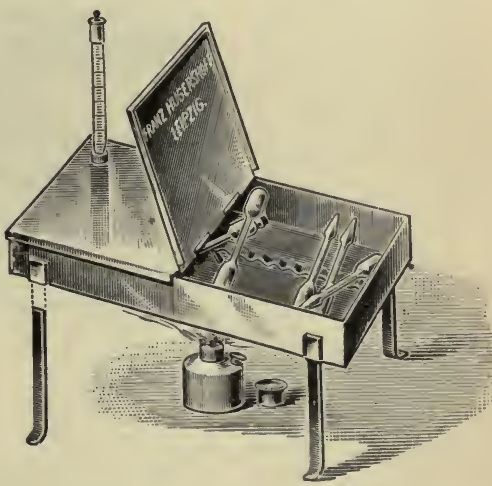

FrG. 35.-Water-bath for Gerber's Method. per pipette and allowed to

1 The amyl alcohol is best obtained from the firm from which the apparatus is purchased. 
flow slowly on to the acid, so that the two liquids mix as little as possible. Finally the amyl alcohol is added, which

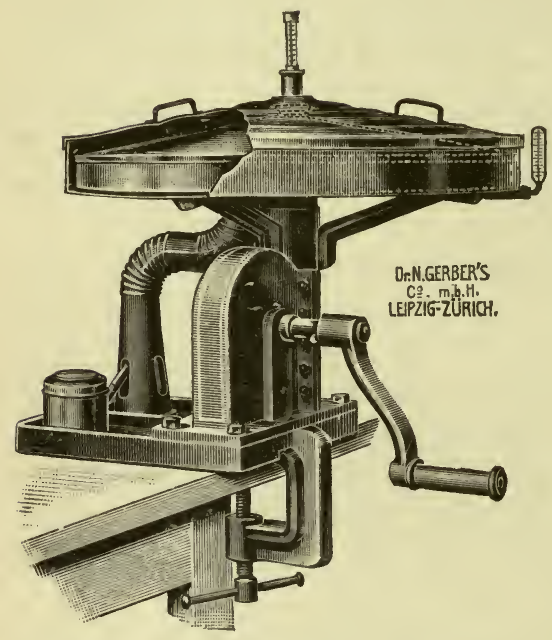

FiG. 36.-Crank·driven Centrifuge with Heating Arrangement. is conveniently done with the help of the apparatus shown in Fig. 33 or in Fig. 38, both of which obviate the necessity of sucking up the strongsmelling liquid. In the latter, the pipette is sunk in the amyl alcohol as far as the 1 c.c. mark, and by simply closing the tube with the finger the liquid can be drawn out.

It is important to use the reagents in the proper order, which is-sulphuric acid, milk, amyl alcohol. If the sulphuric acid is followed by amyl alcohol and the milk last, then the result is sometimes incorrect. This has been proved by Richmond and O'Shaughnessy, ${ }^{1}$ and after-

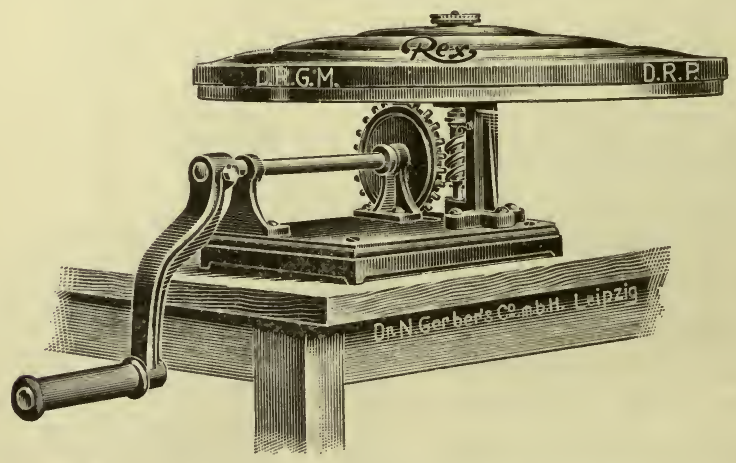

Fra. 37.-Centrifuge without Heating Arrangement.

wards also by Siegfeld ${ }^{2}$ to be due to some unexplained chemical reaction between the sulphuric acid and the amyl alcohol, 
whereby substances insoluble in ether (chiefly amylene, $\mathrm{C}_{5} \mathrm{H}_{10}$ ) were formed.

After the sulphuric acid, milk, and amyl alcohol have been brought together, a rubber stopper, which must not be damaged, is fitted into the mouth of the test-bottle, and then the contents well shaken, the thumb being kept on the stopper to prevent it coming out. As a considerable amount of heat is generated by the action of the sulphuric acid on the milk, the test-bottle should be wrapped in a cloth, or the aluminium sheath designed by Dr. Baechler, of Zurich, may be used.

The shaking of the sample must be done thoroughly and quickly, and the test-bottle inverted several times, so that the liquid in the neck becomes thoroughly mixed. By pressing in the rubber stopper, the height of the liquid can be brought to about the zero point on the scale.

If only a few samples have to be analysed and the room is warm, the test-bottles can be put into the centrifuge without any preliminary heating, otherwise the testbottles must be warmed for a few minutes (not longer) in the water-bath at a temperature of $60^{\circ}-65^{\circ} \mathrm{C}$. When the temperature rises higher than this, say above $70^{\circ} \mathrm{C}$., the rubber stopper is liable to be blown out of the test-bottle. After the test-bottles

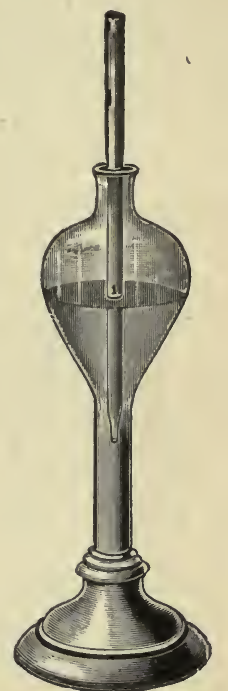

Fig. 38.-Amyl Alcohol Measurer. have been heated they are arranged symmetrically in the centrifuge and whirled for 3-4 minutes at a speed of about 1,000 revolutions per minute. When the centrifuge has a heating arrangement attached to it, the preliminary warming is not, of course, necessary.

When the test-bottles are taken out of the centrifuge they are again placed in the water-bath at a temperature of $60^{\circ}-65^{\circ} \mathrm{C}$., and left there for several minutes before being read; where the centrifuge is heated, the tubes can be read off as taken from the centrifuge.

Bycarefullyscrewing in the rubber stopper, or even by pressing it, the lower limit of the fat column is brought on to one of the 
main divisions of the scale, and then, by holding the test-bottle against the light, the height of the column of fat can be accurately ascertained. The lowest point of the meniscus is taken as the level when reading the upper surface of the fat in a sample of whole milk, and the middle of the meniscus for separated rnilk.

If the column of fat is not clear and sharply defined, the sample must be again whirled in the centrifuge.

Each division on the scale is equivalent to $0 \cdot 1$ per cent., so it is very easy to read to 0.05 per cent., or, with a lens, to 0.025 per cent. If the number which is read off is multiplied by $0 \cdot 1$, then the percentage quantity of fat in the milk is obtained, e.g., if the number on the scale was 36.5 , then the percentage of fat is 3.65 per cent.

If a plug of material forms below the column of fat during the centrifuging, it is a sign that the sulphuric acid is too strong. Siegfeld ${ }^{1}$ recommends in such cases that a weaker acid (sp. gr. 1.800-1.810) should be used. A plug is often obtained when the milk has been preserved with formalin, for this combines with the casein to give a compound insoluble in sulphuric acid. To prevent this, Siegfeld ${ }^{2}$ recommends the addition of 2 c.c. of hydroxylamine solution ( 1 part hydroxylamine hydrochloride and 2 parts water) to 100 c.c. milk, a few drops of ammonia also being added. The amount of fat must then be calculated to the original volume of milk.

If the temperature falls very much during the time the testbottles are being whirled in the centrifuge, all the fat does not separate, so in such cases the centrifuge must be heated in some way. The bottles are cleaned in the same way as those of Babcock or Lindström.

With whole milk, Gerber's test gives excellent results, which agree very closely with those obtained by gravimetric methods of analysis, and it has become almost universal (except in North America) for estimating the percentage of fat in milk.

For the analysis of cream Gerber has proposed the use of a special form of butyrometer, which is open at both ends, and serves also for the determination of fat in butter and cheese. The results, however, which it gives are not reliable, and it is

1 Molkerei-Zeitung, Hildesheim, 1901, p. 797.

2 Zeitschrift f. Untersuchnng der Nahrungs- u. Genussmittel, 1903, p. 397. 
far better to dilute the cream with water and use the ordinary butyrometer.

A. Hesse ${ }^{1}$ has drawn up a table showing the amount of fat corresponding to each division of the butyrometer for dilutions ranging from 1:1 up to 1:5. This table is based not only upon the multiplication of the height of the column of fat by the degree of dilution, but also by the factor 1.03 (the specific gravity of milk), for the specific gravity of diluted cream is about 1 .

Where a balance cannot conveniently be used for weighing out the cream, it may be measured in the following manner. The apparatus which is employed (Fig. 39) consists of a small 50 c.c. round-bottom flask $(a)$, the neck of which is drawn out at $b$, where the mark is placed. In the neck of the flask is fitted a ground hollow stopper provided with a foot and made to hold 5 c.c. The mouth of the hollow stopper is ground and can be closed by a suitable glass disc $(d)$.

The flask is filled to the mark $b$ with water at $17.5^{\circ}$ C., a small funnel being used, so that the sides of the flask above the mark are not wetted. Then the well-mixed cream (the mixing is done by pouring the cream from one vessel to another, avoiding froth as far as can be done), as nearly as possible at a temperature of $17 \cdot 5^{\circ}$,

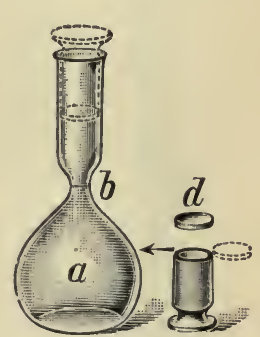

FIG. 39.-Measurer used in Analysis of Cream. is poured into the small measure which the hollow stopper makes until it is quite full and the centre of the cream stands above the level of the sides. The glass disc is then placed on the top, care being taken to exclude any airbubbles. It is thus possible to place the stopper with the adhering disc in the neck of the flask; so that it fits tightly. When the flask is shaken the disc falls off, and the cream mixes with the water. A thorough admixture of the two liquids is assured by reversing the flask repeatedly, the disc which lies above the constricted portion of the flask allowing the air to pass down one side and the liquid up the other, and vice versâ. The results obtained by Gerber's method when testing separated milk are always too low, and the same is found with other 
centrifugal methods, for the fine state of division of the fat makes it difficult for it to separate out. Therefore, when
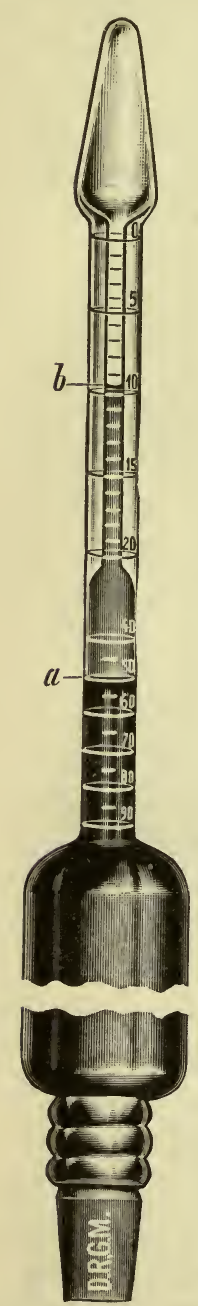

Fig. 40. - Gerber's Precision Butyrometer.

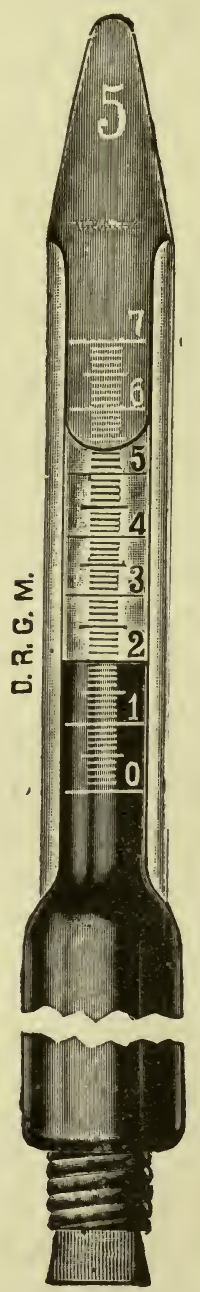

Fig. 41.-Funke's Butyrometer with Flat Scale.

separated (or homogenised) milk has to be analysed, the samples must be taken from the centrifuge after being whirled, put into the waterbath, and then again placed in the centrifuge. If the centrifuge has a heating arrangement, the time of whirling the butyrometers should be increased to 7 or 8 minutes.

Gerber has constructed a so-called "precision butyrometer," which is chiefly intended for the analysis of separated milk. As is seen in Fig. 40, the upper part of the graduated tube is drawn out to a finer bore, so that the divisions on the scale are further apart, and it is easier to read small quantities of fat.

For very exact work with separated milk, or when comparing the efficiency of different separators, Gerber's method is not sufficiently correct, and that of Röse-Gottlieb must be employed. It is accurate enough though for determining whether a separator is working satisfactorily within certain limits. Finally, the butyrometer tubes can be obtained with a flat scale, and as the meniscus of the column of fat then appears more curved, 
and is consequently easier to read, this form is largely used. The butyrometer tubes with flat scale cannot, however, be used for the analysis of separated milk.

\section{Sichler's "Sinacid" Butyrometer" Method.}

A modification in the principle of tests of this class has been introduced by A. Sichler. ${ }^{1}$ In his method, the corrosive and inconvenient sulphuric acid, or in fact any acid, is done away with, hence the name Sinacid (sine acido). In order to destroy the emulsion and to dissolve the casein of the milk, a solution containing sodium phosphate and sodium citrate was at first used. Now the solution used contains sodium hydroxide, Rochelle salt, and sodium sulphate. This mixture of salts, as well as the other chemicals and apparatus required for the test can be obtained from Sichler of Leipsic. $^{2}$

To prepare the solution, $150 \mathrm{~g}$. of the salt are dissolved in 1 litre of water (distilled water not essential). The solution does not undergo any change on keeping, except when it is very cold, and then some of the salt may crystal-

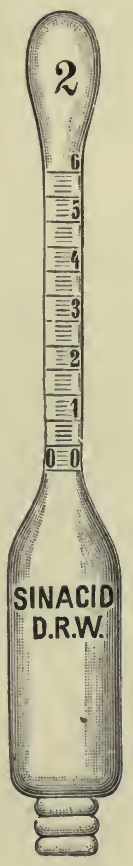

Fig. 42.-Groove Butyrometer for Sichler's Sinacid Method.

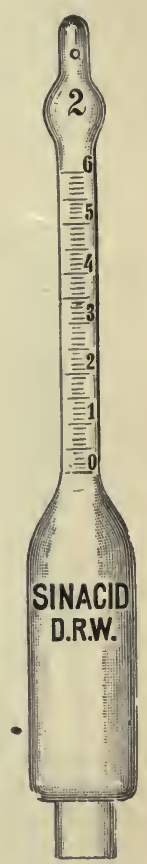

Fig. 43. - Valve Butyrometer for Sichler's Sinacid Method. lise out, but it easily dissolves on warming slightly.

The butyrometer tubes are similar to Gerber's, and are made in two shapes. One is termed the "groove butyrometer" (Fig. $42)$ by the inventor, and the other the "valve butyrometer" (Fig. 43). The former is to be preferred.

To assist the separation of the fat, isobutyl alcohol is used 1 Milch-Zeitung, 1904, p. 417.

2 Lampestrasse, No. 1. 
instead of the unpleasant amyl alcohol, and to make the reading of the column of fat easier the alcohol (sinol) is coloured red, green, or blue.

To make an analysis, the test-bottles are filled, in the following order, with

$$
\begin{gathered}
11 \text { c.c. of Sinacid solution, } \\
10 \text { " milk, } \\
0.6 \text { " sinol, }
\end{gathered}
$$

pipettes of the required size being used. The rest of the operation is carried out as with the Gerber test-bottles, if the "groove butyrometer" is employed. When the "valve

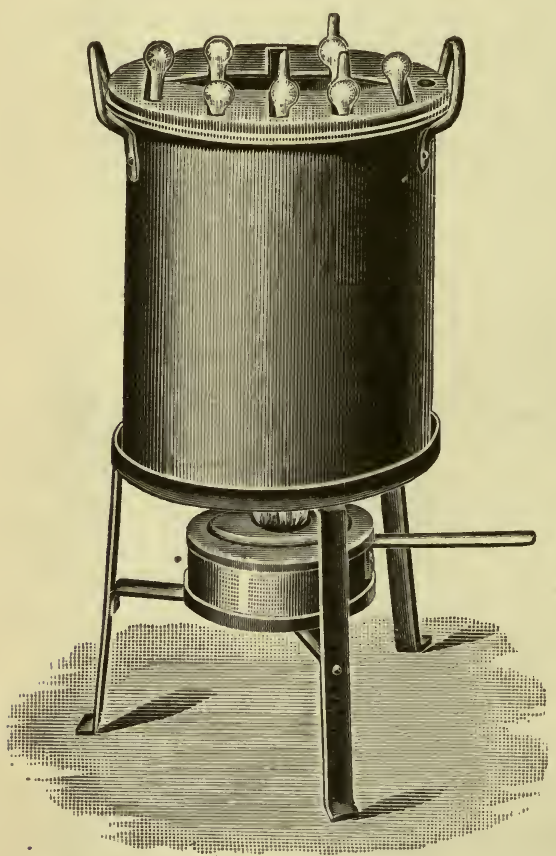

Frg. 44.-Water-bath and Test-bottles. butyrometer" is used, a small piece of rubber tubing must be pushed over the valve before the tube is filled. After the reagents have been filled in, the rubber stopper is fitted into the test-bottle, which is then violently shaken. The test-bottles, after being shaken, are placed in a water-bath having a temperature of $45^{\circ} \mathrm{C}$. Before placing the test-bottle in the water-bath the band of rubber tubing must be pushed upwards so that the small valve is open. This is done to prevent the pressure in the test-bottle rising too high. ${ }^{1}$ The serum should now be perfectly clear, and free from any precipitate. The free alkali of the solution serves to dissolve the casein, whilst the tartrate prevents the precipitation of the lime compounds, and so assists the separation of the fat. The

1 Care must be taken to allow all the liquid in the ball of the butyrometer to flow down into the graduated portion. 
test-bottles are allowed to stay 3-5 minutes in the warm water-bath; they are then shaken and whirled in a centrifuge, Gerber's for example, for 2-3 minutes.

When the tubes are taken from the centrifuge they are again placed in water of a temperature of $45^{\circ} \mathrm{C}$. for a few minutes, and then read in the same way as the Gerber tubes, the column of fat being brought to zero on the scale by carefully screwing in the stopper. The percentage amount of fat can then be read off directly, each division of the scale corresponding to $0 \cdot 1$ per cent.

The cleaning of the test-bottles is very simple; all that is required is to shake them and then to empty whilst warm. They can then be washed with water alone, no soda or brush being required.

This method has undoubted advantages over the acidobutyrometric one, for the corrosive sulphuric acid is not needed and instead of the evil-smelling amyl alcohol, the sinol (which has a pleasant, fruit-like odour) is used. The test-bottles also do not become hot when shaken. According to experiments made by the author, the results are quite equal to those of Gerber's method, and compare very closely with those got by gravimetric analysis. There is no formation of a plug of material below the fat, it being separated sharply, as a beautifully coloured clear column, from the liquid. Equally good results are obtained from sour milk or from milk which has been preserved with potassium bichromate or formalin, provided very large quantities of preservative have not been used. Sour milk should first be made fluid by the addition of a drop or two of strong caustic soda or caustic potash. The method with ammonia does not give satisfactory results.

The Sinacid method in its present form can be regarded as an excellent practical form of determining the percentage of fat in milk. For separated milk, however, it is just as unsuitable as the methods previously mentioned. Test-bottles which have a capillary graduated tube, and are intended for the analysis of separated milk and butter-milk, can be obtained, but with them the results are also unsatisfactory, at least with separated milk. 


\section{Gerber's "Sal" Method.}

Following the principle of the Sinacid method, Gerber ${ }^{1}$ has worked out a modification of his original test, which is known as the "Sal" method. In this method a mixture of sodium hydrate, Rochelle salt, and sodium chloride is used to dissolve the casein, and instead of amyl alcohol the pleasanter isobutyl alcohol is employed. The test is made in the usual Gerber apparatus, and gives results which are quite as good as those got in the acid method.

When using the Sal method, the butyrometer tube must be filled in the following order:

$$
\begin{gathered}
11 \text { c.c. of Sal solution, } \\
0.6 \text { " butyl (isobutyl alcohol), } \\
10 \text { " well-mixed milk, }
\end{gathered}
$$

and the temperature should be about $15^{\circ} \mathrm{C}$.

The rubber stoppers used in the tubes should fit well and be dry, and screwed in so that the liquid reaches to the upper third of the scale. Great care should be exercised in mixing the contents of the tubes, the liquid, after each shaking, being allowed to flow down again into the graduated portion. Then the tubes are put into a water-bath at about $45^{\circ} \mathrm{C}$., allowed to stay there three minutes and again shaken for a short time, and finally put in the centrifuge.

The centrifuge should be whirled for $2-3$ minutes at a rate of 800-1,000 revolutions per minute, and when the tubes are taken out they should be warmed again to $45^{\circ} \mathrm{C}$. and read off. With skimmed, separated, or other milk poor in fat, the reading is taken from the middle of the meniscus, and not from the lower surface as in whole milk.

\section{Determination of the Nitrogenous Substances.}

\section{Estimation of the Total Protein.}

(a) Determination of the Total Nitrogen.

By determining the amount of nitrogen in milk by Kjeldahl's method, and multiplying the result by 6.37 , the quantity of 
protein can be easily, and for most purposes sufficiently accurately, estimated.

About $10 \mathrm{~g}$. of milk are weighed from a small covered beaker, or flask provided with a stopper, into the usual Kjeldahl flask of about 300 c.c. capacity. Then 15 c.c. of concentrated sulphuric acid and a drop of mercury or a little powdered copper oxide are added. To allow of the addition of the same quantity of mercury in each test, Dr. R. Muencke, ${ }^{1}$ of Berlin, has designed a small piece of apparatus, a sketch of which is seen in Fig. 45.

At the commencement the Kjeldahl flask should only be heated gently, but when the water has evaporated the flame can be raised. The heating must be continued until the liquid is quite clear (if mercury has been added) or bright green (if copper oxide has been used); there should be no black particles visible. All the organic nitrogen has now been converted into ammonium sulphate, and the contents of the Kjeldahl flask can be poured into a larger distillation flask, the former being

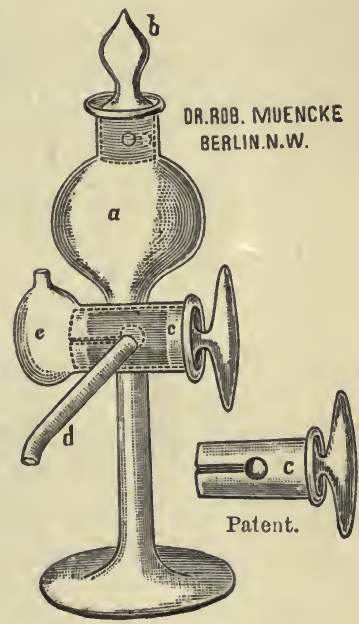

Fig. 45.-Apparatus for Measuring Mercury. afterwards well rinsed out. To the liquid in the distillation flask there is now added 25-30 per cent. of caustic soda or caustic potash solution (the alkali should have been purified by alcohol) until the reaction becomes alkaline, and also enough potassium sulphide solution ${ }^{2}$ (40 g. potassium sulphide per litre) to precipitate the mercury as sulphide, which gives a black colour to the liquid. Finally, a little zine dust is added, and the flask connected immediately to the distillation apparatus, of which there are many forms, one being shown in Fig. 46.

On distillation, the ammonia which has been liberated by the alkali passes over and is led into a flask containing a known

1 Luisenstrasse, 58.

2 If $\mathrm{CuO}$ has been used instead of $\mathrm{Hg}$, the addition of $\mathrm{K}_{2} \mathrm{~S}$ is, of course, unnecessary. 
quantity (40-50 c.c.) $N / 10 \mathrm{H}_{2} \mathrm{SO}_{4}$ along with a few drops of cochineal or lacmoid solution. The point of the distillation tube must always be well below the surface of the sulphuric acid. After three-quarters of an hour all the ammonia has usually distilled over, and the sulphuric acid can be titrated with
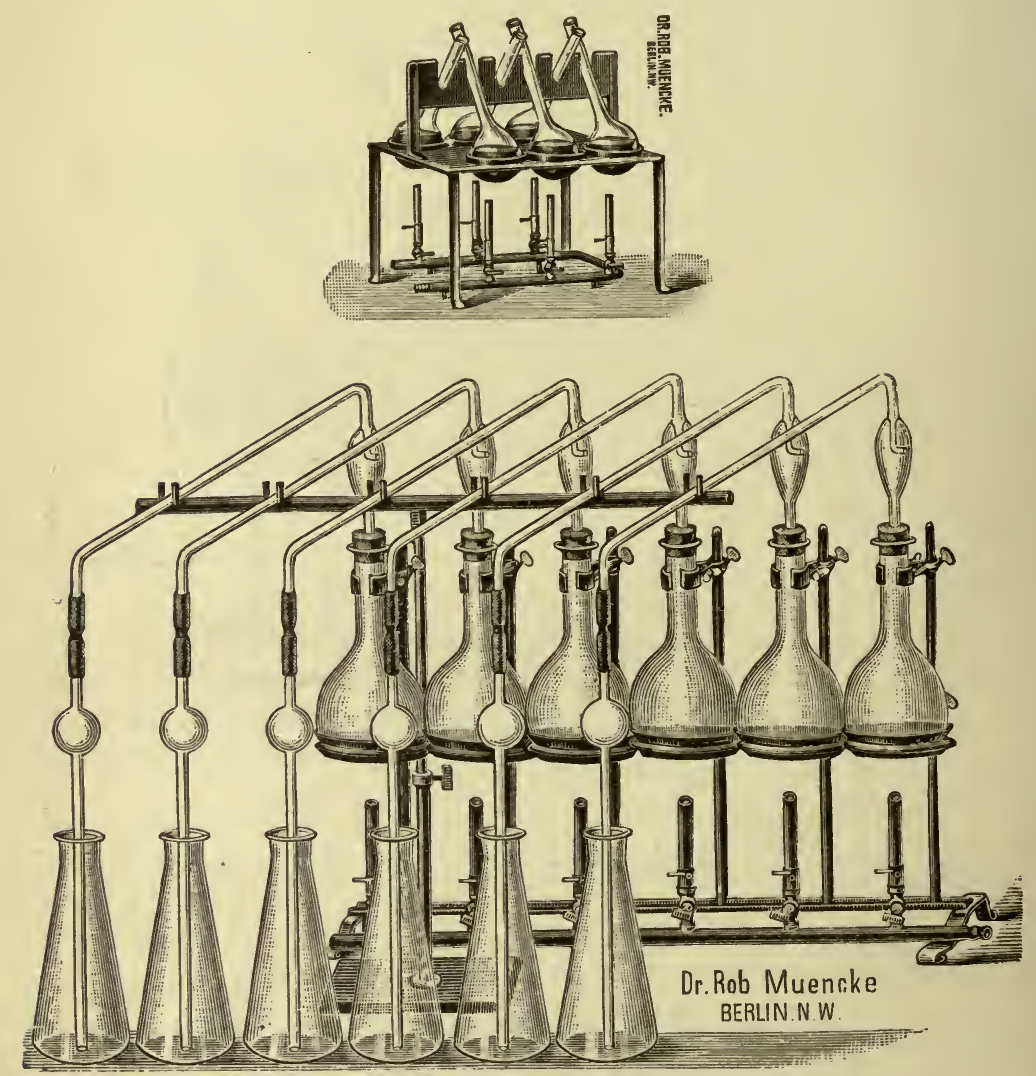

Fig. 46.-Apparatus for Kjeldahl's Method.

$N / 10$ caustic soda or $N / 10$ baryta water, and in this way the nitrogen which has been absorbed as ammonia by the sulphuric acid is estimated. As casein, according to Hammarsten, contains $15 \cdot 65$ per cent. nitrogen, and lacto-albumin $15 \cdot 77$ per cent. according to Sebelien, the amount of protein in milk is obtained by multiplying the nitrogen found by $6: 37$. 
This method is, however, not scientifically correct, for milk contains small quantities of other nitrogenous substances, and these appear in the result as protein. To avoid this error, the following method of analysis may be employed.

\section{(b) Ritthausen's Method for Estimating the Total Protein. ${ }^{1}$}

Twenty-five c.c. of milk are weighed out and diluted with 400 c.c. of water, and to this 10 c.c. of Fehling's copper solution ( 34.63 g. $\mathrm{CuSO}_{4}$ per 500 c.c.) and 6.5 to 7.5 c.c. of caustic potash or caustic soda (14.2 g. $\mathrm{KOH}$ or $10 \cdot 2 \mathrm{~g}$. $\mathrm{NaOH}$ per litre) solution are added. When the precipitate which forms has subsided, the liquid ought to be feebly acid or neutral, but under no circumstances alkaline. Where there is only a slight excess of alkali, the liquid remains cloudy after the precipitate has settled. The liquid is then decanted through a dried weighed filter, the precipitate washed several tirnes by decantation and then brought on to the filter, where it is washed with alcohol to free it from water, and then extracted in a Soxhlet apparatus with ether to get rid of the fat. The residue which remains after the extraction with ether is again washed with alcohol and then dried, first of all in a desiccator over sulphuric acid and then in the drying-oven at $125^{\circ} \mathrm{C}$., or even as high as $150^{\circ} \mathrm{C}$., until the weight is constant. After the precipitate has been weighed, it is ignited, a moderate heat being employed at the commencement. The loss of weight on ignition gives the amount of protein.

This method is, however, not entirely free from error, for some phosphoric and sulphuric acid arising from the phosphorus and sulphur of the protein are weighed along with the ash. ${ }^{2}$ Fleischmann ${ }^{3}$ states that this error for milk containing 3 per cent. protein is 0.114 per cent., for 3.5 per cent. protein 0.133 per cent., and for 4 per cent. protein 0.152 per cent.

Instead of burning the precipitate, the nitrogen in it may be estimated by Kjeldahl's method, and multiplied by 6.37 , to give the amount of protein. If this modification is adopted the precipitate and filter can be heated with sulphuric acid

1 Fresenius, Zeitschrift für analyt. Chem., 1878, p. 241.

2 See note, p. 10.

3 Lehrbuch der Milchwirtschaft, Leipsic, 1907, p. 70. 
without previously being dried, and the nitrogen contained in the filter deducted from the total.

The amount of non-protein nitrogenous substances can be determined either by evaporating the filtrate in a Hoffmeister basin made of thin glass, and then breaking this up and estimating the nitrogen by Kjeldahl's method, or, indirectly, by deducting the nitrogen found as protein from the total nitrogen.

\section{(c) Sebelien's Method for Estimating the Total Protein.}

According to this method, ${ }^{1} 3-5$ g. milk are diluted with two or three volumes of water, a little common salt solution added, and then tannic acid. ${ }^{2}$ The precipitate which falls is washed with cold water and the nitrogen in it determined in the usual way by Kjeldahl's method. Multiplication of the nitrogen by 6.37 gives the protein. This method, which is easy to carry out, gives very good results.

\section{Estimation of Casein.}

The method generally recommended for estimating the casein in milk is to dilute it with water and then precipitate the casein with small quantities of acetic acid, and weigh the dry fat-free material.

Ten to twenty grams are diluted with ten times the quantity of water, warmed to a temperature not exceeding $40^{\circ} \mathrm{C}$., and a few drops of acetic acid added. The coagulated casein is then brought on to a dry weighed filter and washed with water, alcohol, and ether as described above, dried, and weighed.

This method is not, however, very accurate, because it is never certain whether the acetic acid will precipitate the casein quantitatively. The following procedure, due to Sebelien, is more reliable: A weighed quantity of milk is diluted with an equal volume of a saturated solution of magnesium sulphate, and then solid magnesium sulphate added, until the whole is saturated. The liquid is then filtered off, the

1 Zeitschrift für physiol. Chemie, Vol. XIII.

2 Almén's reagent -4 g. nitrogen-free tannic acid +190 c.c. $50 \%$ alcohol + 8 c.c. $25 \%$ acetic acid-may be used. 
precipitate washed with saturated magnesium sulphate solution, and the nitrogen estimated in it by Kjeldahl's method. To obtain the amount of casein corresponding to the nitrogen found, the factor 6.38 is employed.

\section{Estimation of Albumin.}

The albumin in milk can be estimated by simply taking the difference between the amounts of protein and casein, or it is easy to make a direct determination by taking the filtrate got from the precipitation of the casein in Sebelien's method (see above), diluting with water, and precipitating the albumin by means of tannic acid. The nitrogen can then be determined in the precipitate by Kjeldahl's process, and the result multiplied by $6 \cdot 34\left(=\frac{100}{15 \cdot 77}\right)$.

\section{Estimation of the Milk Sugar.}

\section{Gravimetric Methods.}

The gravimetric methods for the estimation of milk sugar are based upon the power which it possesses of reducing an alkaline copper solution (Fehling's solution), whereby an amount of cuprous oxide equivalent to the weight of milk sugar is formed. With milk sugar, however, the relation between the two is not always proportional; a small amount of sugar gives a quantity of cuprous oxide which is relatively smaller than when large amounts of milk sugar are used Soxhlet has therefore constructed a table (Table V in Appendix) in which these discrepancies are corrected. The cuprous oxide is not weighed as such, but converted either into copper or cupric oxide.

\section{(a) Allihn-Soxhlet Method.}

Twenty-five c.c. of milk are weighed out, and treated in exactly the same manner as in Ritthausen's method for estimating the total protein in milk (see p. 83). After the addition of the alkali the liquid should be neutral or slightly acid; it should not under any circumstances have an alkaline reaction. Scheibe's ${ }^{1}$.modification, which is to add $20 \mathrm{cc}$. of a cold

1 Milch-Zeitung, 1901, p. 113. 
saturated solution of sodium fluoride, should also be adopted, for it removes the dissolved lime salts, which otherwise would affect the result of the analysis, from the solution. After the precipitate has been allowed to settle for about half an hour, the flask is filled to the mark and the clear solution filtered through a dry folded filter. Fifty c.c. of Fehling's solution (25 c.c. of copper solution and 25 c.c. of sodium potassium tartrate solution) are heated in a deep porcelain basin, and, when boiling, 100 c.c. of the filtrate previously obtained are added. After the mixture has again been brought to boiling point it is boiled for exactly six minutes, and then filtered at once through an Allihn asbestos filter, which has been previously washed and dried, either in a current of dry air or over the flame, and weighed. The asbestos filter is fitted into a filter flask, which is in communication with a pump (see Fig. 47).

By means of a cork a small glass funnel is fitted on to the mouth of the filter, and during filtration a slight vacuum is maintained. The filter and its contents are washed,

Fig. 47.-Filtration through the Asbestos Filter in the Allihn-Soxhlet Method. first with boiling water, then with alcohol (twice), and with ether (twice).

After the asbestos filter has been dried, it is connected with a hydrogen apparatus, and when the air has been displaced the tube is heated by means of a small flame placed some $5 \mathrm{~cm}$. below it. At the end of a few minutes the reduction is complete, and the asbestos filter tube along with the metallic copper is weighed after it has cooled and a current of air has been drawn through. One mg. copper is almost exactly equivalent to $0.73 \mathrm{mg}$. milk sugar. The table in the Appendix shows the weight of milk sugar equivalent to the weight of reduced copper which has been found. 
By dissolving the copper in nitric acid, washing the tube and asbestos with water and drying them, the filter can be used again. As this method is based upon figures which have been obtained empirically, it must always be carried out in exactly the same way.

\section{(b) Cupric Oxide Method.}

This method of analysis is carried out exactly as the one just described, save that the cuprous oxide is filtered on to a small filter with a known weight of ash. After thoroughly washing with boiling water, the filter is dried in the oven, and the precipitate brought into a platinum crucible. The filter, to which a little cuprous oxide is probably adhering, is burnt in the usual way by the medium of a platinum wire, and the ash added to the precipitate in the crucible. Two drops of nitric acid are then poured on the mass and the crucible heated, at first gently until the nitrate has decomposed, and then more strongly until a red heat is obtained. When all the copper has been converted into cupric oxide, which does not take very long, the crucible is allowed to cool and then weighed. After deducting the weight of the filter ash, the weight of cupric oxide obtained is multiplied by 0.799 , to convert it into copper. An equivalent weight of milk sugar is obtained from Table V.

This method gives as good results as a reduction method, and it is besides simpler and easier to carry out.

\section{Polarimetric Methods.}

The estimation of milk sugar by the polariscope had until recently the reputation of being very unreliable. The cause of this unfavourable opinion is to be sought in the failure to understand the errors to which this method in its original form is liable. Formerly it was believed, and the belief had only very slight foundations, that there was present in the milk a very small quantity of a dextrin-like substance, which influenced the readings of the polariscope, and made them uncertain. This view was upheld in comparatively recent times by v. Raumer and Späth, ${ }^{1}$ but since then A. Scheibe ${ }^{2}$

1 Zeitschrift für angewandte Chemie, Vol. IX, 1896.

2 Ref., p. 85. 
has carefully investigated the sources of error, removed them, and so brought the estimation of milk sugar by the polarimeter again to the fore. Scheibe showed that the previously observed differences were not due to any dextrin-like substance in the milk, but to the use of unsuitable precipitants, and that very satisfactory results could be obtained if the method, which he describes below, was followed:

Seventy-five c.c. of milk are treated with $7 \cdot 5$ c.c. of sulphuric acid (20 per cent. by weight) and 7.5 c.c. of a solution of mercuric iodide, prepared by dissolving $40 \mathrm{~g}$. of potassium iodide in 200 c.c. of water, shaking with $55 \mathrm{~g}$. of mercuric iodide, making up to 500 c.c., and filtering off the unchanged mercuric iodide. The milk which has been treated with the above clarifying solution is made up to 100 c.c., and the filtrate polarised in a $4 \mathrm{dcm}$. tube at $17 \cdot 5^{\circ} \mathrm{C}$. If the Schmidt and Haensch halfshadow polarimeter with double quartz wedge is used, then one saccharimeter degree is equivalent to $0 \cdot 16428 \mathrm{~g}$. milk sugar in 100 c.c. of solution. With polarimeters having a graduated circle and usingsodium light the determination has to be made at $20^{\circ} \mathrm{C}$. and 1 degree in the $4 \mathrm{~d} .-\mathrm{m}$. tube $=0.4759 \mathrm{~g}$. milk sugar in 100 c.c. milk. To eliminate the error due to the volume of the precipitate, either a correction has to be made or the volume of the precipitate determined, and allowance made for it. The determination of the precipitate must never be forgotten in the case of cream or colostrum. The correction to be applied, if the volume of the precipitate is not determined, consists in multiplying the amount of milk sugar by 0.94 in the case of whole milk (2.8-4.7 per cent. fat) or 0.97 in the case of separated milk.

The volume of the precipitate may be found in two ways:

(a) The rotary power of $10 \mathrm{~g}$. pure milk sugar dissolved in 75 c.c. of water treated with 7.5 c.c. of 20 per cent. sulphuric acid and 7.5 c.c. of mercuric iodide solution, and then made up to 100 c.c., is determined. The number of grams of milk sugar which are found in this way correspond to $M_{1}$ in the formula given below.

(b) Ten g. milk sugar are dissolved, with the application of heat, in 75 c.c. of the milk which is to be examined, and, after cooling, 0.5 c.c. concentrated ammonia is added. When the ammonia has acted for 10 minutes, $7 \cdot 5$ c.c. of 20 per cent. 
sulphuric acid and $7 \cdot 5$ c.c. of mercuric iodide solution are added, the liquid made up to 100 c.c., and the filtrate polarised. If now the rotation found with 75 c.c. of milk alone is deducted from that just obtained, then the apparent amount of added milk sugar in grams gives $M_{2}$ of the formula.

(c) The volume of the precipitate is calculated in the following manner: the volume of the liquid and that of the precipitate $(N V)=100$ c.c.; this volume corresponds to $M_{2}$; the volume of the liquid alone $=100-N V$; this corresponds to $M_{1}$ or

$$
\begin{aligned}
100: M_{2} & =(100-N V): M_{1} \\
N V & =\frac{100 M_{2}-100 M_{1}}{M_{2}} .
\end{aligned}
$$

(d) The actual amount of milk sugar in the milk is obtained by multiplying the apparent amount by $\frac{100-N V}{100}$.

\section{The Refractometer Method.}

When Wollny had worked out his method for the estimation of fat in milk by the refractometer, he also proposed to use the refractometer for determining the milk sugar. Wollny also prepared a table for this purpose, but the method was allowed to lie unnoticed until R. Braun ${ }^{1}$ took it up again in 1901 . Braun found that this method, which is the simplest of all, gives results which agree very closely with those obtained by gravimetric analysis. The author of this book can corroborate Braun's conclusions from his own experience.

The way in which the method is carried out is as follows: 5 c.c. of milk are put into the ordinary Wollny test-glass (see Fig. 15, p. 45), and 5 drops of 4 per cent. calcium chloride added. The test-glass is then closed with a cork, wrapped in a cloth for fear of accident, and heated for 10 minutes in a bath of boiling water. The tube is then cooled by being placed in cold water, and then brought to a temperature of $17.5^{\circ} \mathrm{C}$. A drop of the serum which has separated out, and must be quite clear, is brought between the prisms of the refractometer, and the percentage amount of milk sugar can be read off with the help

1 Milch-Zeitung, 1901, p. 578. 
of Table VIII in the Appendix. The temperature at which the reading is made must be exactly $17.5^{\circ} \mathrm{C}$., for it is not easy to apply a correction in this case. Samples of milk which have been treated with preservatives of different sorts can be examined by this method quite as well as fresh milk.

The method, however, is only applicable to cow's milk; that of other animals gives quite false results.

Where a refractometer is present this method is without doubt the simplest that can be employed and is of the greatest assistance where a large number of estimations of milk sugar have to be made at the same time.

\section{VI.-Determination of the Ash.}

About $25 \mathrm{~g}$. of milk are weighed out into a platinum dish and thoroughly dried on the water-bath. ${ }^{1}$ The contents of the dish are then carefully burnt, and the black residue repeatedly extracted with warm water. In this way the mineral substances are separated from the carbon, and the latter can then be ignited until all have disappeared. The water containing the salts is then returned to the platinum dish and evaporated to dry air. The ash which is left is ignited at a low red heat until it becomes perfectly white, and it is then weighed. It is necessary to follow exactly the method given above if concordant results are to be obtained, for if the mass containing carbon is heated very strongly whilst the mineral salts are still present there is certain to be some loss. It is, indeed, difficult to avoid a slight loss, for in the process of ignition the citrates are decomposed, and the mono- and di-phosphates lose water and pass into the meta- and pyro-phosphates. Vieth ${ }^{2}$ has shown that when the ash of milk is ignited at bright red heat for thirty minutes, the chlorine is reduced from 0.09 per cent. to 0.003 per cent.

When the milk has been preserved with potassium bichromate a separate determination of the preservative must be made. This is done after the estimation of total ash, as given above, by means of the following method. ${ }^{3}$ Necessary reagents :

1 Fleischmann recommends the addition of a few drops of acetic acid to prevent a skin forming on the milk.

2 Forschungen auf dem Gebiete der Viehhaltung, \&c., Vol. XV, p. 332.

3 "Arrêté relatif aux méthodes qui devront être employées par les laboratoires agréés pour l'analyse des laits concentrés ou laits desséchés." Journal Officiel du 9 Mars, 1907. 
(1) Ferrous ammonium sulphate solution containing $7 \mathrm{~g}$. per litre, corresponding to $1 \mathrm{~g}$. iron.

(2) Potassium permanganate solution containing $0.5646 \mathrm{~g}$. per litre, equivalent to $1 \mathrm{~g}$. iron. Standardisation of the solution : 20 c.c. of solution (1) are brought together with 5 c.c. pure sulphuric acid and 25 c.c. water into a beaker, and the mixture titrated with the solution of permangan. ate until the faint pink colour persists.

For the actual estimation the ash of milk is brought into a beaker with 25 c.c. water, then 5 c.c. of pure sulphuric acid are added, and 20 c.c. of solution (1). The reduction of the chromic acid follows immediately, and by titrating with permanganate the excess of ferrous ammonium sulphate solution can be determined. The number of cubic centimetres of permanganate solution is subtracted from 20 c.c., and the difference represents the amount of solution (1) required for the reduction of the chromic acid. One c.c. ferrous ammonium sulphate solution is equivalent to $0.000875 \mathrm{~g}$. potassium bichromate.

\section{VII.-Estimation of the Remaining Constituents of MiLK.}

In addition to those constituents of milk already mentioned, there are others, such as citric acid and lecithin, which sometimes have to be determined.

\section{Estimation of Citric Acid.}

The latest and simplest method for estimating citric acid in milk is M. Beau's ${ }^{1}$ modification of that of M. Denigès. ${ }^{2}$ The principle underlying the method is that when citric acid is oxidised with potassium permanganate, acetone dicarboxylic acid, carbon dioxide, and water are formed.

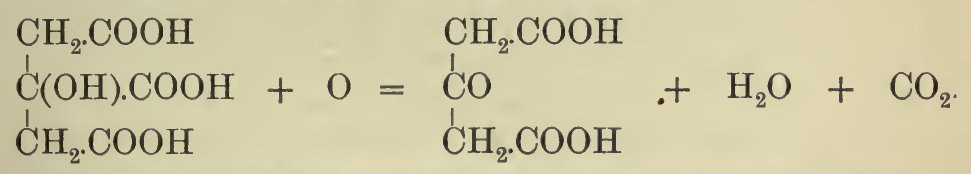

Acetone dicarboxylic acid, on treatment with excess of mercuric sulphate, gives a white precipitate of mercury dicarboxysulphoacetone.

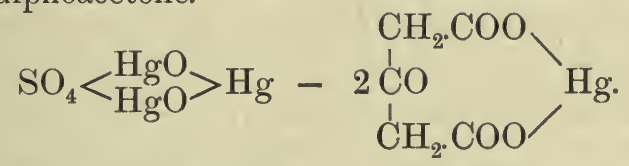

1 Revue Générale du Lait, Vol. III, p. 385.

2 Annales de Phys. et de Chim., Vol. XVIII, 1899. 
This precipitate is insoluble in water, but soluble in concentrated mineral acids, so it is dissolved in hydrochloric acid, excess of ammonia added, as well as a known quantity of $N / 10 \mathrm{KCN}$, and then titrated with $N / 10 \mathrm{AgNO}_{3}$ solution, KI being used as indicator. The difference expresses the amount of $N / 10 \mathrm{AgNO}_{3}$ which is equivalent to the mercury in the precipitate. Beau's ${ }^{1}$ directions for carrying out the estimation are as follows : 50 c.c. of milk are put into a 200 c.c. graduated flask, about 75 c.c. of distilled water and 50 c.c. of mercuric sulphate ${ }^{2}$ are then added, and the flask moderately shaken. This causes the precipitation of the casein, and the flask is then filled to the mark and the contents filtered, the liquid being passed several times through the paper until it possesses only a slight opalescence. It is impossible to obtain an absolutely clear filtrate.

One hundred c.c. of the filtrate (equivalent to 25 c.c. milk) are oxidised with a 1 per cent. solution of potassium permanganate, the liquid being heated to boiling, then the flame removed and permanganate solution added drop by drop, the flask being shaken after each addition. The liquid soon becomes turbid, after which, a yellowish-white precipitate forms. The addition of potassium permanganate is, however, continued until the precipitate sinks rapidly to the bottom, and the liquid above is perfectly clear. To reach this point 5-10 c.c. of potassium permanganate are required. A slight excess is of no disadvantage, for otherwise the oxidation is apt to remain incomplete.

The precipitate has a coffee-brown colour, on account of small quantities of manganese dioxide, but this can be removed by heating the liquid until it boils, then removing the flame and adding small amounts of hydrogen peroxide until the precipitate is quite colourless. Generally five to ten drops of hydrogen peroxide are required for this. After cooling, the liquid is filtered through an asbestos filter (Fig. 47, p. 86), attached to a filter flask and water-pump; the residue being washed with water until the filtrate gives no cloudiness with

1 Loc. cit.

2 The mercuric sulphate is prepared by mixing $50 \mathrm{~g}$. of red oxide of mercury with 400-500 c.c. of distilled water in a litre flask, and then gradually adding sulphuric acid (sp. gr. $66^{\circ}$ Beaumé) until all the mercuric oxide is dissolved. This will take about 75 c.c. of $\mathrm{H}_{2} \mathrm{SO}_{4}$, and the flask is then made up to the mark, heated, and filtered. 
barium chloride. The flask is then changed, and the precipitate dissolved in 10 c.c. of pure concentrated hydrochloric acid, 5 c.c. being used at a time, and the filter washed with 50 c.c. of water after each addition. When the precipitate has been dissolved, the filter flask is again attached to the pump.

The solution, which is cloudy, is now poured into an Erlenmeyer flask, heated to boiling, and again filtered through the asbestos filter under slightly reduced pressure. Operating in this way a perfectly clear liquid ought to be obtained. After washing the filter with distilled water, the solution is again brought into an Erlenmeyer flask and 20 c.c. of ammonia, 10 c.c. of which are to neutralise the acid, are added; also 10 c.c. of potassium cyanide ( $13 \mathrm{~g}$. per litre), which is equivalent to $N / 10$ silver nitrate. Ten drops of a 10 per cent. solution of potassium iodide are used as indicator. The titration is made with $N / 10$ silver nitrate, which is slowly run in until the cloudiness which forms does not disappear on shaking.

Beau has prepared the table given below, from which the amount of citric acid in the milk under examination is directly shown, the quantity of citric acid being expressed in centigrams per litre for each cubic centimetre of $N / 10$ silver nitrate from 0 to 10 . The table is only applicable where the method of operation has been exactly as described above, and where the filtrate $=100$ c.c. obtained from 25 c.c. of milk.

Citric Acid in Mille Corresponding to N/10 Silver Nitrate.

\begin{tabular}{|c|c|c|c|c|c|c|c|c|c|c|}
\hline $\begin{array}{c}\mathrm{N} / 10 \\
\mathrm{AgNO}_{3} .\end{array}$ & $0 \cdot 0$. & $0 \cdot 1$. & $0 \cdot 2$. & $0 \cdot 3$. & 0.4 & 0.5. & 0.6 . & 0.7 & 0.8 & 0.9 \\
\hline 0 & 0 & 3 & 6 & 9 & 12 & 15 & $17 \cdot 5$ & 20.5 & $23 \cdot 5$ & $26 \cdot 5$ \\
\hline 1 & 29.5 & 32.5 & 355 & 38.5 & $41 \cdot 5$ & $44 \cdot 5$ & 47 & 50 & 53 & 56 \\
\hline 2 & 59 & 62 & 65 & 68 & 71 & 74 & $76 \cdot 5$ & 79.5 & $82 \cdot 5$ & $85 \cdot 5$ \\
\hline 3 & $88 \cdot 5$ & $91 \cdot 5$ & $94 \div 5$ & $97 \cdot 5$ & 100.5 & 1035 & 106 & 109 & 112 & 115 \\
\hline 4 & 118 & 121 & 124 & 127 & 130 & 133 & 135.5 & 138.5 & $141 \cdot 5$ & 144.5 \\
\hline 5 & $147 \cdot 5$ & 150.5 & 153.5 & $156 \cdot 5$ & $159 \cdot 5$ & 162.5 & 165 & 169 & 172 & 175 \\
\hline 6 & 178 & 181 & 184 & 187 & 191 & 194 & 197 & 200 & 203 & 207 \\
\hline 7 & 210 & 213 & 216 & 219 & 223 & 226 & 229 & 232 & 235 & 239 \\
\hline 8 & 242 & 245 & 248 & 251 & 255 & 258 & 261 & 264 & 267 & 270 \\
\hline 9 & $273 \cdot 5$ & 276.5 & $279^{\circ}$ & $282 \cdot 5$ & $285 \cdot 5$ & $289 \cdot 5$ & 292.5 & $295 \cdot 5$ & $298 \cdot 5$ & $301 \cdot 5$ \\
\hline 10 & $305 \cdot 5$ & $307 \cdot 5$ & 311.5 & 314.5 & $317 \cdot 5$ & $321 \cdot 5$ & 325 & 328 & 331 & $334 \cdot 5$ \\
\hline
\end{tabular}




\section{Estimation of Lecithin.}

The older methods for determining the amount of lecithin in milk were based upon the extraction of the lecithin with a mixture of alcohol and ether, evaporation of the extract to dryness, fusion with soda and saltpetre, estimation of the phosphoric acid as magnesium pyrophosphate, and multiplication of the result by 7.27. These methods, however, did not give reliable results, for some of the phosphates in the milk were also extracted, and, further, a part of the organic phosphorus was not oxidised during fusion. The factor $7 \cdot 27$ is also unreliable, for the composition of the lecithin of the milk is not perfectly understood.

Bordas and Rackowski ${ }^{1}$ have worked out a newer method for the estimation of lecithin in milk, and it is easier and more accurate than the older methods. The way in which it is carried out is as follows: 100 c.c. of milk are dropped into a mixture composed of :

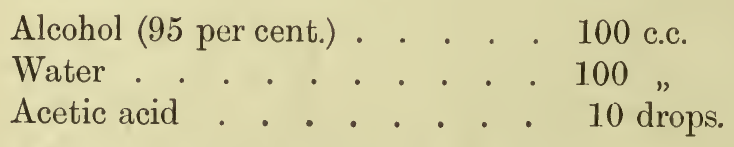

the whole being kept well stirred. In this way a coagulum is obtained which can easily be separated by filtering. After the filtrate has ceased to drop from the funnel, a piece of rubber tubing provided with a pinchcock is drawn over the tip of the funnel. Fifty c.c. of warm absolute alcohol are then poured over the coagulum on the filter, allowed to stay there two or three minutes, the mass being carefully stirred with a platinum spatula, and then the pinchcock is opened and the alcohol allowed to flow away. This process is repeated twice more with 50 c.c. of alcohol, so that in all 150 c.c. of alcohol are employed.

The alcohol is distilled from the filtrate, and this is followed by evaporation to dryness to get rid of the last traces of alcohol. The residue which is left after the evaporation is extracted with a mixture of equal parts of alcohol and ether, filtered, and the filtrate evaporated on the water-bath to drive off the ether, but not the alcohol. The alcoholic solution is then saponified

${ }^{1}$ Compt. rend. de l'Acad. des Sciences, Vol. CXXXIV, p. 1592. 
with caustic potash, and the soap which forms is decomposed with dilute $\mathrm{HNO}_{3}$, and the liquid filtered off. By boiling the filtrate and then heating it on the water-bath it is brought to dryness, and then 10 c.c. of concentrated $\mathrm{HNO}_{3}$ are added. Powdered potassium permanganate is added to the warm liquid until the pink colour persists for a few moments. Afterwards the manganese oxide which forms is dissolved by the addition of a few drops of a 10 per cent. solution of sodium nitrite. The solution is then boiled to free it of nitrous fumes, and the phosphoric acid determined in the usual way with ammonium molybdate solution. After at least twelve hours, the precipitate is filtered off, dissolved in the smallest possible quantity of $\mathrm{NH}_{4} \mathrm{OH}$, and precipitated with magnesia mixture, and the pyrophosphate weighed in the usual way. The weight of the magnesium pyrophosphate multiplied by 1.5495 gives the quantity of glycero-phosphoric acid contained in the sample of milk.

It has not yet been definitely decided which fatty acid is combined with the glycero-phosphoric acid and the choline to make the lecithin of milk. According to Stoklasa ${ }^{1}$ it is stearic acid (as in yolk of egg), whilst Burow ${ }^{2}$ thinks oleic and butyric acids are present. If the first view is accepted, then the lecithin of milk would have the following formula:

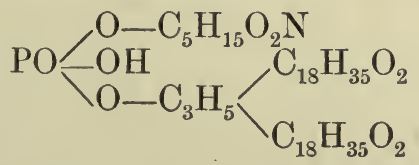

Multiplication of the amount of glycero-phosphoric acid by 4.69 gives the quantity of lecithine in the form of distearin lecithin, whereas multiplication by $3 \cdot 54$ gives it as oleo-butyrin lecithin.

The most important part of the method described above is the oxidation with potassium permanganate, and the more complete this is, the exacter will the results be. Estimated in this way the quantity of glycero-phosphoric acid in milk varies between 0.010 and $0.020 \mathrm{~g}$. per 100 c.c., and is dependent upon the period of lactation.

A more recent method, due to Nerking and Haensel, ${ }^{3}$ gives

1 Hoppe-Seyler's 'Zeitschrift für physiol. Chemie, 1897, Vol. XXIII, p. 343.

2 Ibid., 1900, p. 495.

3 Biochemische Zeitschrift, Vol. XIII, 1908, p. 348. 
equally good results and has the advantage of being easily carried out. One hundred c.c. of milk are precipitated with 200 c.c. of alcohol, the mixture well stirred, and the precipitate allowed to settle. When this has taken place the precipitate is filtered off and the filter and precipitate placed in the cartridge of a Soxhlet extraction apparatus, and extracted for at least thirty hours with chloroform. The alcoholic filtrate is evaporated at $50-60^{\circ} \mathrm{C}$, the residue extracted thoroughly with chloroform, and the extract added to that obtained from the extraction of the filter and precipitate. The united chloroform extract is then allowed to evaporate in a platinum dish, and the residue carefully ignited with a mixture of sodium carbonate and potassium nitrate. After ignition the mass is exhausted with water, the phosphoric acid precipitated with ammonium molybdate, and from the phosphomolybdate the magnesium pyrophosphate is prepared in the usual way. On multiplying the amount of phosphoric acid by $7 \cdot 27$ there is obtained the quantity of lecithin present in the milk. Using this method, Nerking and Haensel found 0.0364-0.1163 per cent. lecithin in cow's milk, the average being 0.0629 per cent.

\section{VIII.-To Distinguish Raw and Heated Milk.}

When it became necessary to require milk coming from herds of cattle suffering from diseases such as foot-and-mouth disease, anthrax, \&c., to be pasteurised, the need of a test which would quickly and simply show whether a given milk had been heated to a certain temperature or not was soon felt. Very numerous indeed have been the methods proposed for this purpose in the last few years, and a very large class of literature ${ }^{1}$ dealing with this subject has accumulated. It is not necessary to discuss all these different methods, particularly as one of the first to be proposed-that by Storch-satisfies all reasonable demands, and for exactness and simplicity is not surpassed by any other.

The methods which have been suggested for the purpose of distinguishing raw and heated milk fall into two main groups :

1 An almost complete record of the early literature is to be found in Weber's "Die zur Unterscheidung roher und gekochter Milch dienenden Untersuchungsmethoden, u.s.w." Inaug. Diss., Leipsic, 1902. 
(1) Those based upon the changes which the protein undergoes when milk is heated.

(2) Those which start from the presence of oxidising enzymes in the milk.

It has been proved that only those reactions which belong to the second category are applicable in practice.

\section{(a) Arnold's Guaiacum Test.}

Arnold ${ }^{1}$ was the first to utilise the oxidising power which milk possesses to prove whether the milk had been heated or not. For this purpose Arnold used tincture of guaiacum. If the resin of the guaiac tree (Guaiacum officinale) is dissolved in an organic solvent, or if the wood of the tree is extracted, then either of these solutions becomes of a dark blue colour under the action of oxidising materials. As was seen in the Introduction (p. 12), cow's milk contains several ferments or enzymes, and it is one of these-peroxidase-which causes ordinary milk to turn blue on the addition of tincture of guaiacum. If the milk, however, has been heated from $78^{\circ}$ to $80^{\circ} \mathrm{C}$., the enzyme is destroyed, and there is no coloration with tincture of guaiacum.

The test is applied as follows: A small quantity of milk is put into a test-tube, and about ten drops of a 5 to 10 per cent. tincture of guaiacum poured into it. Arnold and Menzel ${ }^{2}$ say the tincture of guaiacum is best prepared by dissolving the resin in acetone. In a few minutes after the guaiacum has been added to the milk a blue ring makes its appearance at the junction of the two liquids, but if the milk has been heated to $80^{\circ} \mathrm{C}$. there is no reaction. Before making a test the solution of guaiacum must always be tested to see if it reacts with unheated milk, for the purchased tinctures often behave irregularly.

The peroxidase only oxidises in the presence of peroxides, and the oxygen which is liberated from the peroxide by means of the enzyme is able to oxidise any easily oxidisable substance. In some tinctures of guaiacum organic peroxides are found as a result of auto-oxidation, and such solutions cause the blue colour to appear in milk. In other solutions again, particularly

1 Jahresbericht der Königlichen Tierärztlichen Hochschule, Hannover, $1880-1882$, p. 161.

2 Milch-Zeitung, 1902, p. 247. 
with freshly prepared ones, there are no peroxides, and, in consequence, no blue coloration is given. This fault can of course be remedied by adding a peroxide when making the test, and the most suitable substance is a few drops of hydrogen peroxide.

Instead of tincture of guaiacum, Dupoy, ${ }^{1}$ has proposed an aqueous solution of guaiacol. If the milk has not been heated, then a distinct orange coloration is given if 1 c.c. of aqueous guaiacol solution (1 per cent.) and one drop of hydrogen peroxide (3 per cent.) are added to 1 c.c. of the milk. When the milk has been heated to at least $80^{\circ} \mathrm{C}$. there is no coloration.

\section{(b) Storch's Paraphenylene Diamine Test.}

$\mathrm{V}$. Storch ${ }^{2}$ makes use of the property which milk possesses of decomposing hydrogen peroxide as was discovered by Babcock $^{3}$ in 1889. The course of the decomposition is, according to Babcock and Russell, a tryptic ferment which they call Galactase (see p. 12). Latterly other enzymes have been shown to exist in milk, namely Catalase and Peroxidase, and to these has been ascribed the power of decomposing hydrogen peroxide. The author ${ }^{4}$ is of the opinion that it is the innumerable white blood corpuscles, the so-called leucocytes, in the milk which are the carriers of the enzymes that decompose hydrogen peroxide, and give colour reactions with guaiacum, paraphenylene diamine, \&c. When milk is separated some of the leucocytes pass into the cream, but the greater part remain in the separator slime, of which they form an important constituent $^{5}$ (see Fig. 48). Later investigations have proved the truth of this view, although it must be allowed that the micro-organisms in the milk greatly assist in producing catalase.

Storch found that of all the reagents which come into question for proving if a milk has been heated to $80^{\circ} \mathrm{C}$., the best was paraphenylene diamine. This compound gives ordinarily a brown colour when acted upon by "active" oxygen, but

1 Journal de pharmacie et de chimie, 1897, Vol. I, p. 397.

240 Beretning fra den Kgl. Vet. og Landbohöjskoles Laboratorium for landökonomiske, Forsög, 1898.

${ }^{3}$ Bulletin No. 18, Agric. Expt. Stat., Univ. of Wisconsin, 1889.

4 Nord. Mej.-Tidning, 1899, No. 16. Ref., Milch-Zeitung, 1899, No. 31.

5 Chr. Barthel. "Sur la composition de la boue de centrifuge," Revue Gén. du Lait, Vol. I, p. 193. 
in the presence of casein, that is in milk, the colour is a beautiful indigo blue.

To carry out the test about 5 c.c. of milk are put into a testtube and one drop of a 0.2 per cent. solution of hydrogen peroxide is added from a dropping bottle, also two drops of a 2 per cent. solution of paraphenylene diamine, also from a dropping bottle. ${ }^{1}$ The test-tube is then well shaken, and if the milk has not been heated above $78^{\circ} \mathrm{C}$., or if not heated at all, then an intense blue coloration is produced. If at once or after half a minute the milk becomes bluish-grey, it indicates that it has been heated to a temperature of $78^{\circ}-80^{\circ} \mathrm{C}$. When the colour of the milk is unchanged after addition of the reagents, it may be concluded that the heating has exceeded $80^{\circ} \mathrm{C}$.

Storch's test has shown itself to be the most reliable of all the methods proposed for distinguish-

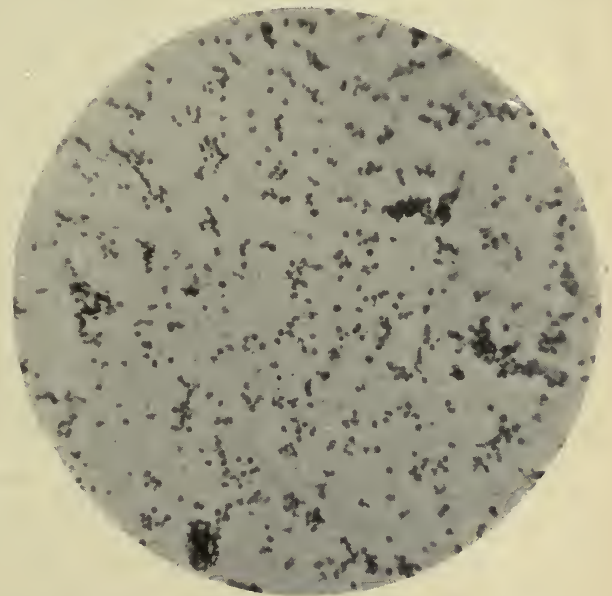

Fic. 48.-1 : 80. Barthel photo. Microphotograph of Leucocytes in Separator Slime. ing heated from un-

heated milk. All the so-called improvements which have been advocated by other chemists have proved to be of no benefit, often indeed the opposite.

If during the pasteurisation of the milk the temperature falls below $80^{\circ} \mathrm{C}$. for a time, then the whole of the milk after being mixed reacts to Storch's test. The sensibility of the test is so great that the admixture of 10 per cent. of milk which has only been heated to $78^{\circ} \mathrm{C}$., suffices to make the wholc volume of milk react to the test.

Reference will be made to the use of this test for proving whether cream which is to be used in making butter has been

1 Paraphenylene diamine solution does not usually remain active for longer than two months, so it must be renewed within that time. 
heated to $80^{\circ} \mathrm{C}$. or not, in the section dealing with the chemical examination of butter (p. 141).

\section{D.-Proofs of Milk Adulteration.}

The most important and at the same time the most difficult use of milk analysis is in proving the adulteration of milk. It is naturally of the greatest importance that such a valuable and at the same time easily-damaged food as milk should be delivered to the consumer in a pure and unadulterated form. Not only because the abstraction of cream and the adulteration with water diminish the food value of the nilk, but because there is great danger in the latter case of the germs of infectious and contagious diseases being introduced into the milk, and so disseminated.

In order to be able to judge if a milk has been adulterated or not, it must first of all be made quite clear of what normal, unadulterated milk is composed. Here, however, grave difficulties present themselves at once, for it is very hard to say within what limits the composition of the milk shall be held to lie. As was noticed in the Introduction, the constituents of milk, and particularly the fat, may vary considerably in quantity in the case of an individual cow, or in the single milkings of various animals. It is therefore essential not to make the limits of normal milk too narrow, otherwise there is the danger of a milk of abnormal composition, although proved to be unadulterated, being regarded as having undergone falsification. In work of this kind, it is better to occasionally allow a guilty person to escape than to punish the innocent.

It has, however, been necessary for the control of the milk trade to fix certain limits for the composition of milk, and these limits refer to the specific gravity of milk, the amount of dry matter, and the percentage of fat, and they vary in different districts. $^{1}$ In general, ordinary mixed milk may be taken as varying as follows:

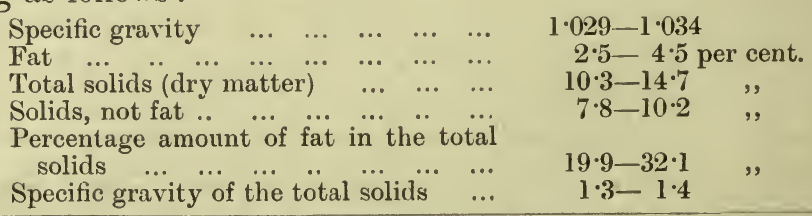

${ }^{1}$ See A. Reinsch, Die gesetzliche Regelung des Milchverkehrs in Deutschland, Hamburg, 1903. 
The mean composition of cow's milk, and the variations in different districts are, however, so uncertain, depending as they do upon the breed, climatic and other conditions, that the most satisfactory way, without doubt, is to determine the average composition of the milk of a district and the fluctuations to which it is subject. To do this, a very large number of milk analyses, made by competent persons, would have to be collected in that particular district.

\section{I.-The Addition of Wa'ter.}

\section{The Nitrate Test.}

Fuchs ${ }^{1}$ was probably the first to recommend the detection of added water in milk by testing it qualitatively for nitric acid. He assumed, which is hardly correct, that the water from most wells and supplies contains small quantities of nitric acid. As a test for nitric acid or nitrates, Uffelmann ${ }^{2}$ used the wellknown reaction with diphenylamine and sulphuric acid, which give a blue coloration in the presence of $\mathrm{N}_{2} \mathrm{O}_{5}$. Schrodt ${ }^{3}$ showed that pure milk does not contain the slightest trace of nitric acid or nitrates even after the food has been mixed with considerable quantities of saltpetre. Two methods of employing the "nitrate test" are as follows :

\section{(a) Soxhlet's ${ }^{4}$ Nitrate Test.}

One hundred c.c. of milk are boiled after the addition of 1.5 c.c. of a 20 per cent. solution of calcium chloride, then to a small portion of the filtrate as many drops of a 2 per cent. solution of diphenylamine in concentrated sulphuric acid are added as will impart a milkiness. Two c.c. of the liquid which has been prepared in the above way are then poured into about an equal quantity of concentrated sulphuric acid contained in a test-tube in such a way as to keep the liquids separate. In the presence of nitrites or nitrates, a blue ring forms at the junction of the two liquids. Where the quantity of nitrates is about

1 Vierteljahrsschrift für öffentliche Gesundheitspflege, 1880, p. 253.

2 Zeitschrift für analytische Chemie, Vol. XXIII, p. 429.

3 Jahresbericht der Milchwirtschaftlichen Versuchsstation Kiel, 1884--1885; see also Henseval and Mullie, Revue Gén. du Lait, Vol. IV, p. 512; and Marcas and Huyge, Ibid., Vol. V, p. 355.

${ }^{4}$ Herz, Die Kuhmilch, p. 49. 
$0.5 \mathrm{mg}$. per 100 c.c., the blue colour appears after a few minutes; where the amount is less, say about $0.1 \mathrm{mg}$. in 100 c.c., several hours may be needed.

This test gives good results, but the method is too complicated to make its use general in practice.

\section{(b) Fritzmann's ${ }^{1}$ Nitrate Test.}

Fritzmann does not use diphenylamine, but shows the presence of nitrates in milk by formalin and sulphuric acid.

One drop of a 10 per cent. solution of formaldehyde is added to 10 c.c. of milk and about 10 c.c. of chemically pure sulphuric

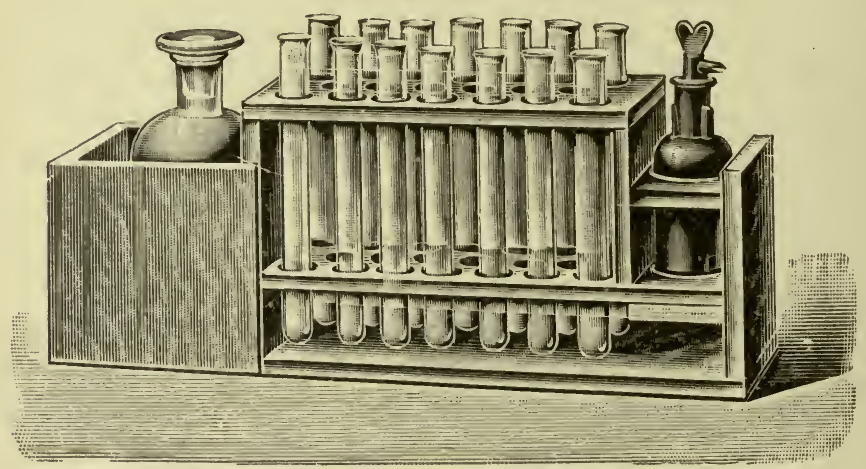

FIG. 49. - Apparatus for making the Nitrate Test.

acid (sp. gr. 1.815), and the presence of nitrates in the milk is indicated by a bluish-violet colour, only shown in the presence of protein.

Siegfeld ${ }^{2}$ carries out the test by not mixing the two liquids, but allowing the milk, to which a drop of formalin has been . added, to form a layer above the acid. Any violet coloration then becomes apparent at the junction of the two liquids. Made in this way, it is claimed by Siegfeld that the test is more sensitive than with diphenylamine.

Fritzmann and Reiss have arranged a testing apparatus, which they call "Hydro," for the use of those dealing in milk.

1 Zeitschrift für öffentliche Chemie, 1897, No. 23. Ref. in Milch-Zeitung, 1898 , p. 73.

2 Molkerei-Zeitung, Hildesheim, 1902, No. 10. 
It consists of a small box containing dilute formalin solution, concentrated sulphuric acid, test-tubes, and a very dilute solution of nitric acid.

Fritzmann's test has the advantage over that of Soxhlet that it can be applied directly to the milk and by anybody.

Care must, however, be taken not to draw definite conclusions from the results of the nitrate test. Many spring and tap waters contain no nitrates, so that milk adulterated with such water would not give the nitrate reaction. On the other hand, the washing of the vessels with water containing a lot of nitrates would cause the milk to react to the nitrate test, although no adulteration had taken place.

A positive result got by the nitrate test can only be regarded as an indication that the milk is suspicious, and must be carefully examined. Under no circumstances, however, should a positive result be regarded as a certain proof that water has been added to the milk.

\section{Determination of the Specific Gravity, the Fat, and the 'T'otcel Solids.}

Fleischmann ${ }^{1}$ has, with the help of very comprehensive and accurate analytical data, constructed the formula which bcars his name, and which serves to calculate the amount of total solids in milk if the specific gravity and the percentage of fat are known. Provided the total solids and the specific gravity are known, the amount of fat in a sample of milk can be determined. This formula, in which the specific gravity of the dry matter free from fat (solids not fat) is taken to be 1.6007 , is as follows:

$$
t=1.2 . f+2.665\left(\frac{100 s-100}{s}\right)
$$

$t=$ total solids expressed in percentages.

$f=$ percentage of fat in milk.

$s=$ specific gravity of the milk at $15^{\circ} \mathrm{C}$.

If $t$ and $s$ are known, and $f$ is to be calculated, this may be done with the help of the formula:

$$
f=0.833 . t-2 \cdot 22\left(\frac{100 s-100}{s}\right)
$$

1 Journal für Landwirtschaft, 1885, Vol, XXXIII, p. 251. 


\section{IO4 MILK AND DAIRY PRODUCTS PART}

Halenke and Möslinger have worked out the following simplified formula:

$$
t-\frac{f+s / 5}{0 \cdot 8}
$$

where $s$ denotes lactometer degrees, not the specific gravity.

The safest and most generally used method for proving the addition of water to milk is to determine the specific gravity of the milk, the fat, and the total solids. This last is obtained most conveniently by using Fleischmann's formula, which, it may be noted, applies only to fresh milk. When the milk is old very considerable differences are found between the calcu-

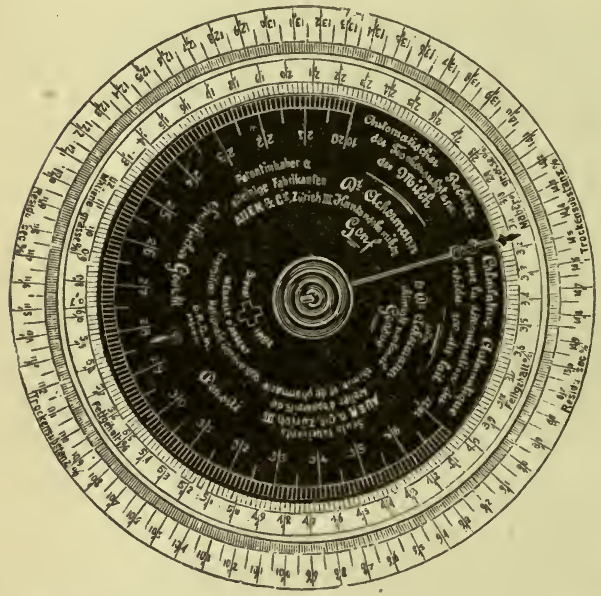

Fig. 50.-Ackermann's Apparatus for Calculating the Total Solids.

lated and the real amounts of total solids.

Ordinary normal mixed milk has only very exceptionally a specific gravity below 1.029 (equal to $29^{\circ}$ of the lactometer). If water is added to the milk the specific gravity naturally sinks, the fall being considerable, as much as three lactometer degrees, for each 10 per cent. of added water. If, for example, normal milk has a density of 30.6 lactometer degrees, it would only have 27 degrees after the addition of 10 per cent. of water, and 24.5 degrees after the addition of 20 per cent. A specific gravity of 28 degrees, consequently makes a sample of milk suspicious, and if the specific gravity is less than the above figure, water has certainly been added. If, therefore, the lactometer indicates the addition of water, the percentage of fat in the milk should be determined by some reliable method, and then the amount of total solids can be ascertained with the help of Table VII. in the 
Appendix. Dr. Ackermann has constructed an apparatus, ${ }^{1}$ shown in Fig. 50, which is very useful where a number of calculations have to be made. If it is found that the total solids are below the usual limit of 10.3 per cent., then it may be concluded with almost absolute certainty that there has been an addition of water. In order to have a further check upon the result the amount of total solids minus fat, which $=t-f$, as well as the percentage of fat in the total solids, which is $100 \mathrm{f} / \mathrm{t}$, should be considered.

If the specific gravity, the fat contents, the total solids, and the solids minus fat are abnormally low (see the data on p. 100) then the milk has been adulterated with water. The percentage amount of fat in the total solids undergoes, of course, no change by the addition of water.

\section{Determination of the Specific Gravity of the Milk Serum.}

Often when milk is suspected of being adulterated with water a determination is made of the specific gravity of the milk serum, because this is very constant for at least three days after the milk has been drawn. ${ }^{2}$ As different numbers are obtained for the specific gravity of milk serum according to the different methods of obtaining the serum, Bialon ${ }^{3}$ has proposed to use for such calculations a number which is independent of the total solids and of the milk serum. This number is the specific gravity of a hypothetical fat-free milk, $\sigma$, which is got from the formula:

$$
\sigma=\frac{100 s-f}{00-f / 0.933}
$$

in which $s$ is the specific gravity of the milk, $f$ the percentage amount of milk which it contains, and 0.933 the average specific gravity of the milk fat.

If the number $\sigma$ sinks below 1.0323 the milk is to be suspected, for this number is the lowest that has been obtained in a long series of estimations of the specific gravity of fat-free milk.

1 Made solely by Auer \& Co., Zurich.

2 Reinseh und Lührig, Zeitschrift für Untersuchung der Nahrung.s- und Genussmittel, 1900, p. 521 .

3 Milchwirthschaftliches Zentralblatt, Vol. I, 1905, p. 363 ; see also J. Nisius, "Über das spezifische Gewicht und den prozentischen Wassergehalt des Milchplasmas," Milch-Zeitung, 1902, No. 27. 


\section{Determination of the Freezing Point of Milk.}

J. Winter ${ }^{1}$ in his investigations upon the freezing point of the different fluids of the animal organism found that the freezing point of milk was constant at $0.555^{\circ} \mathrm{C}$. with very slight variations. On the addition of water the freezing point approaches nearer and nearer the freezing point of water. Winter proposed that the freezing point of the milk should serve as a test for the presence of added water. It must be noted, however, that the freezing point of milk depends upon the degree of acidity, and the sourer the milk becomes the lower does the freezing point become. Winter considers, in spite of this, that a determination of the freezing point and of the degree of acidity would serve as a simple method for controlling the adulteration of milk with water.

Although Winter's proposal has not yet found a very extended application the operation, as worked out by him, ${ }^{2}$ may fitly find a place here. Where a large number of samples have to be tested they are placed in cylindrical corked vessels in icewater in order to bring the temperature near to $0^{\circ} \mathrm{C}$.

The samples are taken from the ice-water and placed in a freezing mixture composed of one part of coarse salt and three to four parts of finely-crushed ice. After the thermometer, which is fastened to a stand and is divided in $0.01^{\circ} \mathrm{C}$., has been placed in one of the samples, the milk is stirred with a ringshaped stirrer until the column of mercury remains still. This temperature is noted and is a little lower than the ordinary freezing point, in the vicinity of which, however, it lies. With distilled water the temperature which is at first given is correct, and denotes the freezing point of the thermometer.

When a determination of the freezing-point of milk (and solutions in general) is made, the first reading must, however, be corrected. This is done by taking the cylinder out of the freezing mixture and allowing it to gradually become warmer, at the same time keeping it well stirred. If the stirring is done with the right hand and the cylinder held in the left, then the warming is much more rapid, thanks to the heat

1 Compt. rend. de l'Acad. des Sciences, Nov., 1895.

2 Winter et Parmentier. "La cryoscopie du Lait," Revue Gén. du Lait, Vol, III, p. 193 , 
of the hand. In this way the large pieces of ice soon begin to melt. When the temperature has risen five- or sixhundredths of a degree the cylinder is returned to the freezing mixture before all the frozen milk has melted. The fall of the mercury must now be carefully watched, and the stirrer raised and sunk again twice each second. After a few seconds the column of mercury stands still, so if there is no movement of it after the stirrer has been raised twice or three times the temperature may be read off. After one or two minutes another reading should be taken, and the thermometer should then show the same temperature as before. If this is the case, then the reading may be taken as correct.. Sometimes, but not often, the temperature rises half- to one-hundredth part of a degree in this time, and if so this latter reading is the correct one. Should the temperature, however, fall during this pause of a few minutes, then the determination must be made afresh.

In each series of cryoscopic tests a correction of the zero has, moreover, to be made. This is because each thermometer alters slightly for two or three years after being made. The glass shrinks as it were, and so the bulk of the thermometer becomes smaller, and the zero point is consequently raised. Where the measurements have to be so carefully made as in the present case, the change cannot be neglected.

The correction is made simply by determining the freezingpoint of distilled water, and the position of this on the scale is taken as the zero.

Winter ${ }^{1}$ has made use of the fact that a lowering of the freezing-point, $\Delta$, is almost proportional to the addition of water, and has constructed the following formula:

$$
E=V \frac{a-\Delta}{a},
$$

where $E$ is the volume of water which has been added to the milk, $V$ the total volume of water and milk, $a$ the normal lowering of the freezing-point of milk $\left(0.55^{\circ} \mathrm{C}\right.$.), and $\Delta$ the observed lowering of the freezing-point.

The following table, which has been drawn up by Winter, permits of the percentage of added water being directly ascertained from the lowering of the freezing-point:

${ }^{1}$ Bull. de la Societé Chim., 1895, Vols. XIII-XIV, No. 24, p. 1,102. 


\begin{tabular}{|c|c|c|c|}
\hline Observed $\Delta$. & Water, per cent. & Observed $\Delta$. & Water, per cent. \\
\hline 0.53 & $3 \cdot 63$ & 0.44 & $20 \cdot 00$ \\
\hline 0.52 & $5 \cdot 45$ & 0.43 & $21 \cdot 81$ \\
\hline 0.51 & $7 \cdot 27$ & 0.42 & $23 \cdot 63$ \\
\hline $0 \cdot 50$ & $9 \cdot 09$ & 0.41 & $25 \cdot 45$ \\
\hline 0.49 & $10 \cdot 90$ & $0 \cdot 40$ & $27 \cdot 27$ \\
\hline $0 \cdot 48$ & $12 \cdot 72$ & $0 \cdot 39$ & $29 \cdot 09$ \\
\hline 0.47 & $14 \cdot 54$ & $0 \cdot 38$ & $30 \cdot 90$ \\
\hline 0.46 & $16 \cdot 36$ & 0.37 & $32 \cdot 72$ \\
\hline 0.45 & $18 \cdot 18$ & $0 \cdot 36$ & $34 \cdot 54$ \\
\hline
\end{tabular}

This method of determining the amount of added water is very simple, and perfectly reliable if carried out carefully. It is remarkable that it has not found more general application, for it not only shows that water has been added, but gives also the amount with àccuracy.

The author ${ }^{1}$ has experimented with this method, and is quite satisfied that the results are reliable, and that, in agreement with Winter and Parmentier, ${ }^{2}$ Schnorf, ${ }^{3}$ and others, pure. unadulterated milk never has a higher freezing-point than $-0.54^{\circ} \mathrm{C}$. This is independent of breed, individuality, food, stage of lactation, sexual excitement, amount of fat, \&c., but a very small addition of water at once raises the freezing-point.

\section{Determination of the Refractive Index of Milk Serum.}

With a refractometer it is also possible to detect added water, but if accurate results are to be obtained exactly the same method must be used in obtaining the serum. It is of importance to remember that the serum will vary according to whether it is got from milk that has soured naturally, or that which has been treated with acetic or other acid or acted upon by rennet. In all these cases the results vary somewhat, and the serum obtained in these ways must be separated by filtration, which is always difficult and takes time. Ackermann's ${ }^{4}$ method, which is carried out in the following manner, gives the best results :

Thirty c.c. of the milk to be examined are placed in a glass tube provided with a reflux condenser, and then 0.25 c.c. of calcium chloride solution added. This solution of calcium

1 Revue Générale du Lait, Vol. IV, p. 505.

2 Loc. cit.

${ }^{3}$ Neue physikalisch-chemische Untersuchungen der Milch, Zurich, 1905.

+ Zeitschr. für Unt. der Nahr-und Genussmittel, Vol. XIII, 1907, p. 186. 
chloride must have a sp. gr. of exactly $1 \cdot 1375$, and when diluted to $1: 10$ should show a refraction of $26^{\circ}$ at $17 \cdot 5^{\circ} \mathrm{C}$.

The contents of the tube is then well shaken, the reflux condenser, which should be $22 \mathrm{~cm}$. long, is attached, and the tube placed in boiling water in a water-bath. For use where a series of analyses have to be made, Ackermann has devised an apparatus in which twelve to twenty-four glass tubes can be heated (Fig. 51). ${ }^{1}$

In order to obtain a proper serum preparation, the boiling of the water when the tubes are placed in it should be interrupted for as short a time as possible. This is best achieved by the use of a large Fletcher burner. Boiling for fifteen minutes is sufficient, and the tubes are then withdrawn and placed in a vessel containing cold water; after some little time the temperature of the water is raised to $17 \cdot 5^{\circ} \mathrm{C}$. The small amount of water which condenses in the upper end of the tube in which the coagulation is taking place, and in the condenser, is mixed with

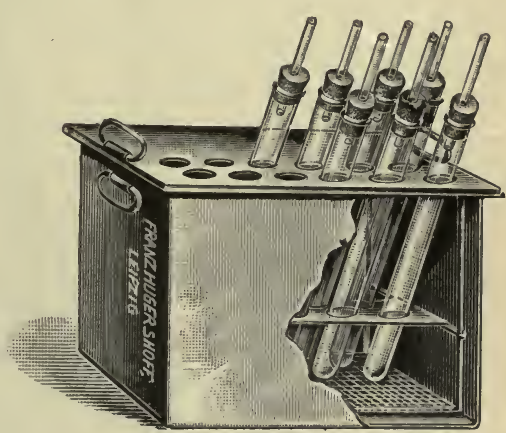

FI.. 51. -Water-bath for Ackermann's Method.

the serum without shaking. The serum can now be poured off into a small beaker and used for the refractometer determination; filtration is not necessary.

Ackermann found as a mean of 2,800 investigations with this method that the variations in the refractive index of normal milk lay between 38.5 and 40.5 with the Zeiss immersion refractometer.

The influence of water in milk is shown in the following table by Ackermann :

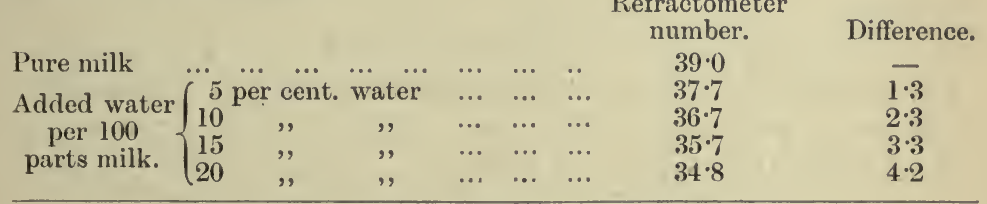

1 To be obtained from Franz Hugershoff, Leipsie. 
Added water $\left\{\begin{array}{cccccc}25 \text { per cent. water } & \ldots & \ldots & \ldots \\ 30 & , & , & \ldots & \ldots & \ldots \\ \text { per } 100 & & & \\ \text { parts milk. } & , & , & \ldots & \ldots & \ldots \\ 40 & , & , & \ldots & \ldots & \ldots \\ 45 & , & , & \ldots & \ldots & \ldots \\ 50 & , & , & \ldots & \ldots & \ldots\end{array}\right.$

Refractometer number.

$34 \cdot 0$

$33 \cdot 3$

$32 \cdot 6$

$32 \cdot 0$

$31 \cdot 4$

$30 \cdot 9$
Difference.

$5 \cdot 0$

$5 \cdot 7$

$6 \cdot 4$

$7 \cdot 0$

$7 \cdot 6$

$8 \cdot 1$

Mai and Rothenfusser, ${ }^{1}$ who, contrary to Henkel, ${ }^{2}$ obtained very good results with Ackermann's method, give the following detailed directions, when using the refractometer. Before taking

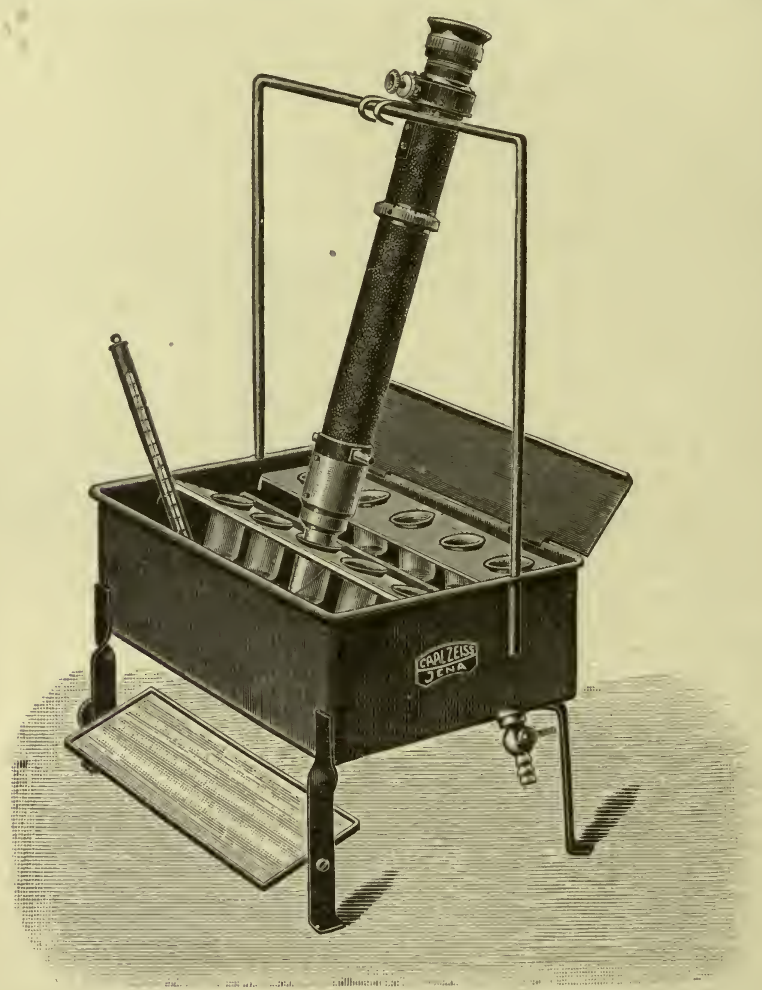

FiG. 52.-The Immersion Refractometer.

the reading the beaker should stand for at least eight minutes in a water-bath, having a temperature of exactly $17.5^{\circ} \mathrm{C}$. During this time, and also during the reading, great care should be exercised to keep every trace of water from the serum, for

1 Molkerei-Zeitung, Berlin, No. 4, 1909.

2 Ibid., No. 52, 1908. 
the fraction of a drop of water in the contents of a beaker at onee lowers the refractive index. The prism of the immersion refractometer should also be suspended in the bath, so that it can acquire a temperature of $17.5^{\circ} \mathrm{C}$. The refractometer is then taken from the stand, the prism thoroughly dried with a soft cloth, then dipped for a moment in the first serum glass and afterwards again dried. Then the prism is completely immersed in the serum, and the degree of refraction read, the instrument being held in one hand and the vernier manipulated with the other. When the reading has been noted, the refractometer is returned to the stand. The temperature of the bath must be controlled from time to time, for the thermometer must be kept at exactly $17 \cdot 5^{\circ} \mathrm{C}$. The larger the bath, the easier it is, of course, to keep the temperature constant, and the firm of Zeiss, in Jena, have constructed a bath to hold forty samples for the above-mentioned investigations. From time to time the refractometer should be tested to see if at $17.5^{\circ} \mathrm{C}$. the refractive index of distilled water is exactly at fifteen divisions of the scale.

Naturally, the Zeiss-Wollny milk refractometer already described can be used for these determinations.

It is clear that the refractometer tests are particularly to be recommended where the addition of water to milk has been proved and the exact amount has to be determined.

\section{II.-The Removal of Cream.}

A determination of the specific gravity of a sample of milk often serves to indicate the addition of water, but no such indication is afforded by the specific gravity when part of the cream has been removed.

The only safe method which is available is to estimate the pereentage amount of fat in the total solids after the quantity of the latter has been calculated, with the help of Fleischmann's formula, from the specific gravity and the fat-contents. The fact that the specific gravity is shown to be normal, whilst the amount of fat is low, must arouse attention, for under natural conditions a low specific gravity implies a small percentage of fat, and vice versâ.

When part of the cream is removed, or the milk diluted with separated milk, there is in each case a change in the percentage 
amount of fat in the total solids, the reduction being more or less according to circumstances. If the specific gravity has been found to be normal, but the fat, on the other hand, too low, then there is cause for suspecting the removal of cream. After the amount of total solids, which under the conditions in question is very low, has been ascertained from Table VII, $a$ and $b$, the percentage quantity of fat in the total solids is calculated from the formula: $p=100 \mathrm{f} / \mathrm{t}$.

If the percentage amount of fat in the total solids is less than 19.9 (see p. 100), it is practically certain that the milk has been adulterated either by removal of cream or by addition of separated milk. The weight of solids not fat under such circumstances is often greater than with unadulterated milk.

\section{Simultaneous Removal of Cream and Addition of Water or Separated Milk.}

This kind of adulteration is generally easier to detect than the simple removal of cream, although the specific gravity alone does not afford any certain indication provided the falsification has been cleverly done. The specific gravity may then remain normal, and a determination of the fat, the total solids, and the percentage amount of fat in the latter are necessary to prove the adulteration. In such cases the quantity of fat and of total solids is abnormally low, and the percentage amount of fat in the total solids is also lower than under normal circumstances.

\section{The Stall Test.}

Recourse is had to this method of tessting a milk when the analytical results point to adulteration having been practised in one or other of the above-mentioned ways. In order that such a test may be of value, it is not only necessary to know from which herd of cattle the milk has come, but also whether the milk was morning's, evening's, or mixed from all the cows in the herd or only from a few of them, \&c. When these particulars have been obtained, then a sample of milk should be taken in such a manner as to obtain exactly the conditions under which the suspected one was procured. If, for example, the suspected example was taken from milk drawn at 5 o'clock on the evening before, then the test sample should be procured 
twenty-four hours later. Should this be impossible, then another day must elapse-in all, forty-eight hours-and under certain circumstances it may be necessary to wait until the evening of the third day. If the suspected sample is from mixed morning's and evening's milk, then the stall sample must likewise be taken from similarly mixed milk.

In cases of this sort the assumption is made that the mixed milk of several cows and of the same milking (morning or evening) does not alter in solids not fat from day to day. This assumption is, however, not always to be relied upon, but the exceptions are very few indeed where there has been no change in the food or in the treatment of the cows from the time of taking the milk which was analysed to the time of taking the sample in the stall.

The stall sample must be taken in the presence of two impartial witnesses, one of whom takes his stand near to the churn into which the whole of the milk is poured, whilst the other stands beside the milker and sees that the can is empty when the milking begins and that the cows are milked clean. After an average sample has been taken, it is sealed up by the witnesses and sent, with any remarks they have to make, to the analyst. If now the analysis shows the milk to be of normal composition, whilst the previous sample from the same animals and similar time of milking was abnormal and indicated adulteration, either by addition of water, removal of cream, or both, there is no doubt that such has been practised.

It is only in the case of considerable adulteration that the fraud can be constated with certainty without the help of the stall test. In all other cases it is difficult to arrive at a safe conclusion without taking a sample from the stall. There is the further advantage in taking this stall sample that the degree of addition of water or removal of cream can be calculated with its help.

\section{Calculation of the Added Water.}

When calculating the amount of water added to milk, it is best to start from the amount of solids not fat, for these are subject to relatively smaller variations than are the specific gravity and particularly the quantity of fat. 
F. J. Herz ${ }^{1}$ has constructed the following formula from which the amount of added water can be calculated:

$$
x=\frac{100}{r^{\prime}}\left(r-r^{\prime}\right):
$$

$x=$ water added to 100 parts of the milk.

$r=$ percentage of solids not fat in the stall sample.

$r^{\prime}=\quad " \quad " \quad$ " $\quad$ suspected sample.

The addition of water can also be calculated with a fair degree of accuracy from Ambiihl's formula:

$$
x=\frac{s-s^{\prime}}{s} \cdot 100:
$$

$$
\begin{aligned}
& s=\text { the specific gravity of the stall sample. } \\
& s^{\prime}=" \quad " \quad \text { suspected sample }
\end{aligned}
$$

Less than 10 per cent. of added water is often difficult to prove, especially if the amount of the addition does not exceed 6 per cent. Where more than 10 per cent. of water has been added there is no difficulty in securing absolute proof with the aid of the above formulæ.

2. Calculation of the Amount of Cream Abstracted.

The following formula by Herz serves to show this:

$$
y=f-f^{\prime}+\frac{f^{\prime}\left(f-f^{\prime}\right)}{100}:
$$

$y=$ the fat removed as cream from 100 parts of milk.

$f=$ the amount of fat in the suspected sample.

$f^{\prime}=\quad, \quad " \quad$ stall sample.

3. Calculation of Added Wuter and Abstracted Cream in Same Sample.

Into this calculation the amount of water in the milk, and also its contents in fat and solids, enters. Böhmländer has given the following formulæ for the purpose:

$$
\begin{gathered}
x=\frac{r^{\prime}}{r^{\prime}}\left(w^{\prime}-w\right): \\
y=100\left(1-\frac{f^{\prime} r^{\prime}}{f r^{\prime}}\right):
\end{gathered}
$$

1 Chemiker-Zeitung, 1893, Vol. XVII, p. 836. 
$: ;=$ quantity of added water.

$y=$ abstracted cream, expressed in the amount of fat removed fiom 100 parts of milk.

$r=$ solids not fat in the stall sample.

$r^{\prime}=\quad " \quad, \quad$ " suspected sample.

$v=$ percentage amount of water in the stall sample.

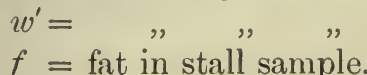

$f=$ fat in stall sample.

$f^{\prime}=\quad " \quad$ suspected sample.

It need hardly be mentioned that analyses of this description only have a legal value when they are made by fully competent persons, and the greatest care exercised both in the actual analyses and in the taking of the samples of milk.

\section{Admix'ure with Goat's Milk.}

In some districts cow's milk is occasionally mixed with small quantities of goat's milk, a process which must be regarded as falsification if the purchaser is not aware of it.

R. Steinegger ${ }^{1}$ has worked out a simple method for distinguishing between cow's and goat's milk, and for proving the addition of large quantities of the latter to the former.

Steinegger found that when 25 per cent. ammonia is added to goat's milk there is a precipitation of protein material which does not occur with cow's milk, because the casein from cow's milk goes into solution.

Twenty c.c. of milk are heated to $50^{\circ}$ C. and allowed to stand at this temperature. Then 2 c.c. of ammonia (25 per cent.) are added, and the whole well shaken, the shaking being repeated each half-hour or hour for two or three hours. At the end of this time a layer of protein matter has formed under the layer of cream, although the protein is specifically heavier than the milk. The reason of the protein substances being there is because they have been carried up by the rapid rising of the cream. If such a precipitation has formed, it is evidence that the milk is from the goat, or is cow's milk largely mixed with goat's.

Where the milk contains about 20 per cent. of goat's milk, the

1 Landwirtschaftliches Jahrbuch der Schweiz, 1904. 
precipitate is about 2 c.c.; where 50 per cent. is present, the test-tube is almost completely filled with the precipitate. An addition of less than 20 per cent. of goat's milk cannot be proved with certainty by this method. Steinegger has modified his method latterly, and now separates the milk in a small separator, so that it contains less than 0.3 per cent. of fat. The addition of ammonia now gives a precipitate which sinks to the bottom of the tube, and it is much easier to distinguish it and to estimate the amount.

The method cannot be used for milk more than 24 hours old, nor for colostrum, or milk mixed with colostrum. It cannot be used either for the milk of sick and diseased animals, nor, in fact, for abnormal milk of whatever origin.

\section{The Detection of Artificial Colouring.}

The Institute for Hygiene in Hamburg has worked out the following method for the detection of artificial colouring matter in milk or cream : One hundred to two hundred c.c. of milk or cream are made faintly acid with acetic acid, or are allowed to sour spontaneously, and then warmed to $80^{\circ} \mathrm{C}$. The curd, which contains fat and the colouring substance in addition to the protein matter, is separated from the serum by filtration through a cloth, digested twice with water to get rid of milk sugar, and then well pressed. The moist curd is then boiled repeatedly with alcohol until no colour remains, and the different lots of alcohol used for the extraction are evaporated down to 10 or 20 c.c. To the residue an equal quantity of absolute alcohol is added, and the whole left in the ice-chest for twelve hours. At the end of this time the solution will only contain a little fat, and any colouring matter which is present will be noticeable. A strip of filter paper is now dipped into the liquid, which rises slowly by capillary attraction to the height of the edge of the beaker and then evaporates.

When the milk is pure a faint yellow or brown band of colour forms on the upper part of the paper, whereas any added colouring matter causes a more pronounced shade, varying from pink to deep orange, to form below the band given by pure milk. The strip of paper is freed from fat by washing with 
petroleum ether, which does not attack the colouring matter or the paper fibre.

In this way many of the usual colouring substances used in the dairy can be detected, but there are several that are not.

\section{Addition of Saccharate of Lime.}

Latterly it has become a not uncommon practice to add saccharate of lime, which is sold under various names, to milk and cream. This material preserves the milk or cream and also increases their viscosity, so giving them the appearance of being richer in fat. Cream treated in this way is more easily whipped, and it has also a deeper yellow colour. Naturally such an addition must be regarded as an adulteration, and Baier and Neumann ${ }^{1}$ have worked out a good method, which has been well tested by Liihrig. ${ }^{2}$

\section{The Detection of Saccharose in Milk to which Saccharate of Lime has been added.}

Twenty-five c.c. of milk or cream are shaken in a small Erlenmeyer flask with 10 c.c. of a 5 per cent. solution of uranium acetate, allowed to stand for five minutes, and filtered through a folded filter.

The filtrate, as a rule, is perfectly clear, and does not need to be poured through the filter again. To 10 c.c. of the filtrate (with cream the total filtrate is usually not more than 10 c.c., so all of it must be used) 2 c.c. of a cold saturated solution of ammonium molybdate are added, and 8 c.c. of $\mathrm{HCl}(1$ part 25 per cent. acid to 7 parts water). This mixture is then shaken, and placed in a water-bath at $80^{\circ} \mathrm{C}$. for five minutes. At the end of this time the presence of saccharose in the milk or cream is indicated by the solution becoming more or less blue, according to the amount of saccharose present. If the test-tube is allowed to remain for a longer time in the water-bath the blue colour becomes still deeper. At the end of ten minutes it is deep blue, whereas with unadulterated milk the colour at the end of

1 Zeitschrift für Untersuchung der Nahr. und Genussmittel, 1908, Vol. XVI, p. 51 .

2 Molkerei-Zeitung, Hildesheim, 1909. 
five minutes is faintly green, which deepens, but never acquires the characteristic blue shade obtained in the first case.

By means of this method quantities of saccharose smaller even than 0.05 per cent. can be detected. ${ }^{1}$

\section{The Detection of Lime in Milk.}

Two hundred and fifty c.c. of milk at $15^{\circ} \mathrm{C}$. are shaken with 10 c.c. of hydrochloric acid (10 per cent.) and allowed to stand for half an hour at ordinary temperature. After this the mixture is filtered, and the first portion of the filtrate returned to the filter. The filtration proceeds very slowly, and the filter should be covered to prevent evaporation, just as is done in the preparation of milk serum.

One hundred and four c.c. of the filtrate, equivalent to 100 c.c. of milk, are poured into a 200 c.c. flask, 10 c.c. of 10 per cent. ammonia added, and the flask filled to the mark with water at $15^{\circ} \mathrm{C}$. After standing for half an hour the solution is filtered through a folded filter, the first portion of the filtrate being collected separately and poured back on to the filter. One hundred c.c. of this filtrate, equivalent to 50 c.c. of milk, are tested with 10 c.c. of 5 per cent. ammonium oxalate solution, and the determination of lime made in the usual way, but without warming the liquid.

Where cream is to be examined the procedure is as follows:

Two hundred c.c. of cream at $15^{\circ} \mathrm{C}$. are shaken with 8 c.c. of a 10 per cent. solution of hydrochloric acid and left to stand for half an hour, after which the serum can be filtered through a folded filter, the usual precautions being taken to collect the first portions separately and return them to the filter. Then 52 c.c. $\left(\frac{20}{4} \frac{8}{)}\right)$, equivalent to 50 c.c. of cream, are transferred to a 100 c.c. flask, 5 c.c. of 10 per cent. ammonia added, the flask made up to the mark and allowed to stand half an hour, then the contents filtered. Five c.c. of the filtrate, equivalent to 25 c.c. of cream, are allowed to stand overnight with 10 c.c. of a 5 per cent. solution of ammonium acetate. The estimation of lime is similar to that described above for milk, and the result must be multiplied by four.

1 Latterly, Rothenfusser (Zeitschr. für Untersuchung der Nahr- und Genussmittel, 1909, Vol. XVIII, p. 145) has worked out a method for the detection of saccharose in milk, which is simpler and gives very good results. Its sensibility is greater than the above-mentioned. 
Baier and Neumann state, and this is corroborated by Liihrig, that in normal milk the lime in the serum is from 13-18 mg. per 100 c.c., whilst in the case of milk or cream to which saccharate of lime has been added the results are always considerably higher.

\section{The Addition of Preselrvatives.}

In most countries the addition of antiseptic or other foreign materials to the milk is forbidden. This is a well-justified course, for, apart from the fact that most preservatives cannot be regarded as non-injurious from a hygienic standpoint, the use of them must militate against the care and cleanliness which ought to be exercised in dealing with milk. The substances which are usually added to milk to increase its keeping properties are carbonate or bicarbonate of soda, boracic acid and its salts, salicylic acid, benzoic acid, formaldehyde, and hydrogen peroxide.

\section{Carbonate or Bicarbonate of Soda.}

Carbonate of soda is not used for its antiseptic properties, for it possesses none, but because of its power of neutralising the lactic acid which forms, and which would ultimately curdle the milk. The development of the bacteria is in no way hindered by the use of this "preservative," which must be regarded as harmful, seeing that it permits of a milk being in an advanced stage of decomposition without the appearance indicating the fact.

The use of carbonate or bicarbonate of soda was much greater formerly, and is still common to a small extent in some parts. Generally, about $1 \mathrm{~g}$. of anhydrous soda is used to each litre of milk.

To prove the addition of carbonate or bicarbonate of soda to a milk, Hilger's method can be used, which consists in diluting 50 c.c. of milk with 250 c.c. of water, heating it, and then precipitating with a small quantity of alcohol. After the solid matter has been filtered off, the filtrate is evaporated to half its former volume and tested for an alkaline carbonate with litmus paper, \&c. 
Soxhlet-Scheibe use another method, which is based upon the quantitative determination of the carbon dioxide in the ash of the milk. The normal amount of carbon dioxide in milk does not exceed 2 per cent.

\section{Boracic Acid and Borax.}

E. Meissl ${ }^{1}$ has given the following method for detecting boracic acid or its salts in milk: 100 c.c. of milk are made alkaline with milk of lime evaporated to dryness and then ignited. Lime is preferable to the alkalies because the ignition is much easier. The ash is dissolved in the smallest possible quantity of concentrated hydrochloric acid, the carbon filtered off, and the liquid evaporated to dryness, care being taken to drive off all the acid. The residue is then moistened with a little very dilute hydrochloric acid, the mass completely soaked with tincture of curcuma, and evaporated down on the water-bath. In the presence of the smallest trace of boracic acid, the residue is coloured red, varying between vermilion and cherry-red. This test is extremely sensitive: $1 \mathrm{mg}$. to $0.5 \mathrm{mg}$. of boracic acid in the ash or 0.001 to 0.002 per cent. in the milk can be detected with certainty.

Concentrated hydrochloric acid also gives a cherry-red colour with tincture of curcuma, but it disappears at once on the addition of water. The ash which has been tested with curcuma can, if required, be used for the flame test. To carry out this test, the material is brought along with methyl alcohol into a flask provided with inlet and outlet tubes. Hydrogen gas is then passed through the flask and lighted, and the flame will have a green colour in the presence of boracic acid.

\section{Salicylic Acid.}

Ch. Girard ${ }^{2}$ uses the following method in testing for salicylic acid in milk: 8 drops of acetic acid and 8 drops of mercuric nitrate are added to 100 c.c. of milk and 100 c.c. of water at $60^{\circ} \mathrm{C}$., and well shaken. The precipitate is filtered off and the filtrate shaken out with ether, which extracts the salicylic acid, the ethereal solution being filtered through a dry filter. After

1 Zeitschrift für analytische Chemie, 1882, p. 531.

2 Ibid., 1883, p. 277. 
the ether has evaporated spontaneously, the salicylic acid is left in the form of colourless crystals which can be dissolved in alcohol and tested with a drop of 1 per cent. ferric chloride solution. The characteristic violet denotes salicylic acid.

\section{Benzoic Acid.}

E. Meiss ${ }^{1}$ recommends the following method for the detection of this preservative : 250-500 c.c. of milk are made alkaline with a few drops of lime- or baryta water, and then evaporated to about a quarter of the bulk. Powdered gypsum is stirred into the remaining liquid until a paste is formed, which is then dried on the water-bath. The gypsum only serves to hasten the drying, and powdered pumice stone or sand can be used equally well. When the mass is dry, it is finely powdered and moistened with dilute sulphuric acid and shaken out three or four times with about twice the volume of 50 per cent. alcohol, in which benzoic acid is easily soluble in the cold, the fat only being dissolved to a very slight extent or not at all. The acid alcoholic liquid from the various extractions, which contains milk sugar and inorganic salts in addition to the benzoic acid, is neutralised with baryta water and evaporated to a small bulk. Dilute sulphuric acid is again added, and the liquid shaken out with small quantities of ether. On evaporation of the ether, the benzoic acid is left behind in almost pure state, the only impurities being small quantities of fat or ash.

The benzoic acid which is obtained is dissolved in a small quantity of warm water, a drop of sodium acetate and neutral ferric chloride added, and the red precipitate of benzoate of iron indicates the presence of the acid.

\section{Formaldehyde.}

Fritzmann's reaction (p. 102) can be employed for the detection of formalin if applied in the reverse order to that used in testing for a nitrate. Ten c.c. of milk. are taken, and a few drops of very dilute nitric acid and also 10 c.c. of chemically pure sulphuric acid (sp. gr. 1.815) are added. The presence of formaldehyde in the milk is indicated by a violet-blue coloration. A simpler and more reliable method is to use Schiff's

${ }_{1}$ Zeitschrift für analytische Chemie, 1882, Vol. XXI, p. 531. 
reagent, which is a solution of fuchsin from which the colour has been discharged by sulphur dioxide. ${ }^{1}$

One c.c. of dilute sulphuric acid $(1: 3)$ is added to 100 c.c. of milk, which is then distilled from a 500 c.c. distillation flask, a ring burner being used, until 20 c.c. of distillate has been collected. A part of the distillate (10 c.c.) is then treated with the above-mentioned Schiff's reagent. If, after 5 or 6 minutes, an intense carmine colour appears, which passes into violet-blue on shaking with 2 c.c. of hydrochloric acid, there was formaldehyde present in the milk.

Simpler, and yet quite delicate, is the method recommended by Utz, in which Winckel's reagent-vanillin and hydrochloric acid-is employed : 10 c.c. of hydrochloric acid (sp. gr. 1.19) and a few grains of vanillin are warmed in a test-tube with 10 c.c. of milk. With ordinary milk a beautiful deep violet coloration is given, but if only traces of formaldehyde are present in the milk the colour is yellow instead of violet.

\section{Hydrogen Peroxide.}

Where so little hydrogen peroxide is added to the milk that it is split up entirely into water and free oxygen, no test will detect the preservative, for it is no longer there. If, on the other hand, a small excess of hydrogen peroxide is present, it can easily be determined both qualitatively and quantitatively.

\section{(a) Qualitative Estimation.}

The method generally recommended in text-books for the detection of hydrogen peroxide by means of potassium bichromate and ether (blue coloration of the ether due to perchromic acid) is not sufficiently sensitive, and is easily surpassed by Arnold and Mentzel's test with vanadic acid. This acid is used in the form of a solution made by dissolving $1 \mathrm{~g}$. of the acid in $100 \mathrm{~g}$. dilute sulphuric acid. Ten drops or this yellow solution are added to 10 c.c. of milk, which turns red if hydrogen peroxide is present. As little as $0.01 \mathrm{~g}$. hydrogen peroxide in 100 c.c. of milk will be shown by this test.

The most sensitive reaction, however, is that of Wilkinson and

1 For the preparation of Schiff's reagent, see p. 232. 
Peters ${ }^{1}$ with benzidine (paradiamidodiphenyl). An alcoholic solution of benzidine acetate gives a deep blue coloration with the oxygen freed from the hydrogen peroxide.

When 4 drops of an alcoholic solution of benzidine ( 4 per cent.) and two drops of acetic acid are added to 10 c.c. milk, the presence of hydrogen peroxide is denoted by a blue coloration. Investigations made by the author show that by this method $0.005 \mathrm{~g}$. hydrogen peroxide in 100 c.c. milk easily be detected.

\section{(b) Quantitative Estimation.}

For the quantitative estimation of hydrogen peroxide in milk the following method can be used. It has been tested by the author, ${ }^{2}$ and very good results have been obtained.

0.5 c.c. of dilute sulphuric acid $(1: 3)$ are added to 25 c.c. of milk, and after the coagulated casein has been allowed to settle completely it is filtered off, and 5 c.c. of the filtrate measured out into a bottle with a glass stopper. Then 10 c.c. of a 10 per cent. solution of potassium iodide and 0.5 c.c. of sulphuric acid $(1: 3)$ are added, the flask shaken, kept in the dark for four hours, the shaking being repeated frequently and the free iodine afterwards titrated with sodium thiosulphate ${ }^{3}$ solution, starch paste being used as an indicator. One c.c. of the thiosulphate solution $=0.0017 \mathrm{~g}$. hydrogen peroxide. The reactions take place as follows :

$$
\begin{aligned}
& \mathrm{H}_{2} \mathrm{O}_{2}+2 \mathrm{KI}=2 \mathrm{KOH}+\mathrm{I}_{2} . \\
& \mathrm{I}_{2}+2 \mathrm{Na}_{2} \mathrm{~S}_{2} \mathrm{O}_{3}=2 \mathrm{NaI}+\mathrm{Na}_{2} \mathrm{~S}_{4} \mathrm{O}_{6} .
\end{aligned}
$$

The method, which is thus based upon the power of hydrogen peroxide to free iodine, cannot be used with the milk itself, for otherwise a compound of iodine and milk would be formed. After coagulation of the casein the serum which remains has no action upon the iodine.

1 Zeitschrift für Untersuch. der Nahr-und Genussmittel, 1908, Vol. XVI, p. 172.

${ }_{2}$ Revue Gén. du Lait, Vol. II, p. 289.

${ }^{3}$ For the preparation of this solution, see p. 154. 


\section{I24 MILK AND DAIRY PRODUCTS PART}

\section{The Fermentation Test with Milk.}

A simple test which can be used to ascertain whether a milk is normal or faulty in a bacterial sense is that based upon the changes which it undergoes when kept at a temperature of $38^{\circ}-40^{\circ} \mathrm{C}$. Under these favourable conditions the struggle for existence amongst the bacteria is clearly indicated by the appearance of the milk at the end of a certain number of hours.

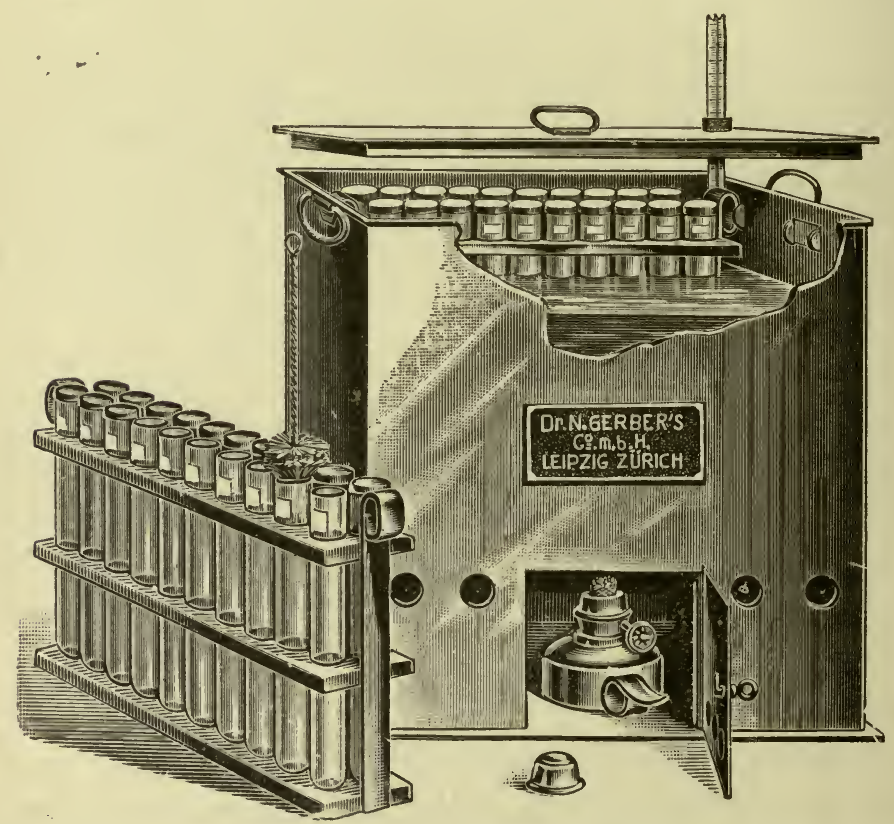

Fic. 53.-Apparatus for the Fermentation Test.

This can be made to serve as a test of the bacterial properties of the milk. The method in its present form is due to Walter, and only requires a simple apparatus such as that shown in Fig. 53.

The cylindrical test tubes, which fit into a metal stand, are furnished with caps of metal or rubber. The tubes are either large, holding 120-140 c.c., or small, holding 40-50 c.c.; the larger are to be preferred, for in them the test is more 
reliable and also easier to judge. The smaller ones have the single advantage of taking up less room, so that a larger number of tests can be made at one time.

The stand containing the test-tubes can be sunk in a square vessel made of tinplate, which holds water and can be heated with a spirit lamp. In the lid of this water-bath there is placed a thermometer, the bulb of which dips into the water.

The most important point to be observed in using the fermentation test is to keep the test-tubes as free from bacteria - that is, sterile-as possible. It is easy to see that if the tubes contain bacteria before the milk is put into them, the changes which take place during the test are due, not to the bacteria of the milk alone, but also to those originally present in the tube. The results in such cases are absolutely worthless. If the care necessary to make the tubes clean and bright is not bestowed upon them, then the test had better not be attempted. It is preferable, of course, to sterilise the glass tubes, which is easily done in a steam steriliser. Where this is not obtainable the tubes should be cleansed with a warm solution of soda, then washed in hot water, and finally rinsed in cold water which has previously been boiled. The tubes may then be placed in an inverted position and left to drain dry.

The test-tubes are numbered, and after milk has been poured into them to within $1 \mathrm{~cm}$. of the top, they are closed by the tin or porcelain covers and placed in the bath, the water of which is at a temperature of $40^{\circ} \mathrm{C}$., and kept there for twelve hours. During this time the temperature of the water is kept constant between $38^{\circ}$ and $40^{\circ} \mathrm{C}$. with the help of the spirit lamp. At the end of the time the samples are taken out, and the appearance, smell, or other property of the milk serves as an indication of its bacterial contents. If the milk is quite normal and fresh, then it ought to undergo no change other than acquiring a pure sour smell. Should the sample be completely and homogeneously coagulated, it is not an indication that the milk is not so good, for the lactic acid bacteria apparently still have the upper hand. This condition points, however, to a considerable length of time having elapsed since the milk was drawn. If, on the other hand, the coagulation of the milk is irregularly flocenlent, and there are large quantities of whey, then there has not been a due regard to cleanliness. 


\section{I26 MILK AND DAIRY PRODUCTS PART}

\begin{tabular}{|c|c|c|c|}
\hline 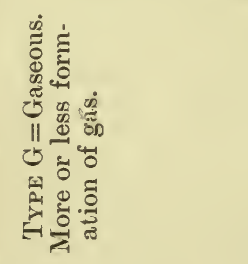 & 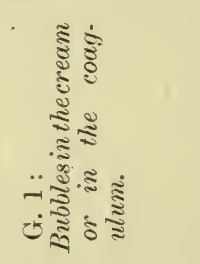 & 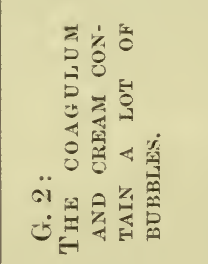 & 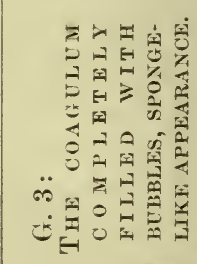 \\
\hline 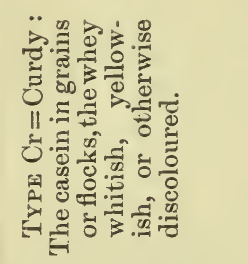 & 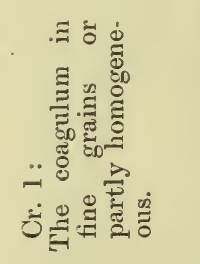 & 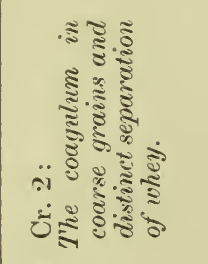 & 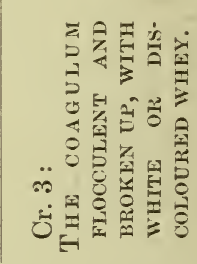 \\
\hline 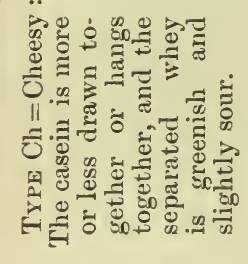 & 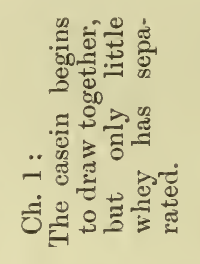 & 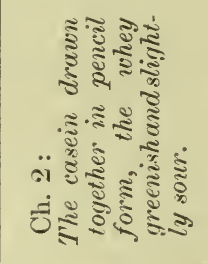 & 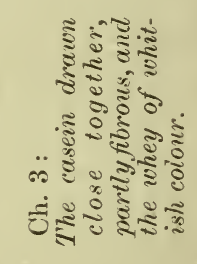 \\
\hline 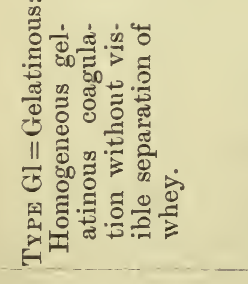 & 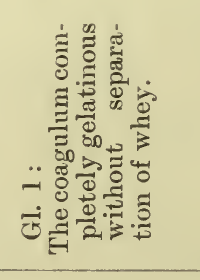 & 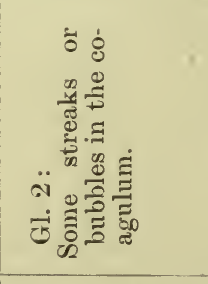 & 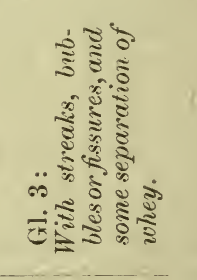 \\
\hline 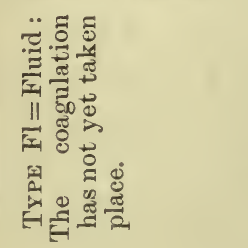 & 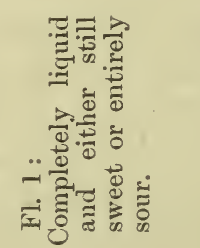 & 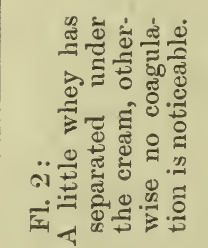 & 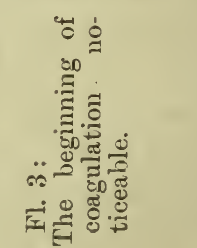 \\
\hline
\end{tabular}




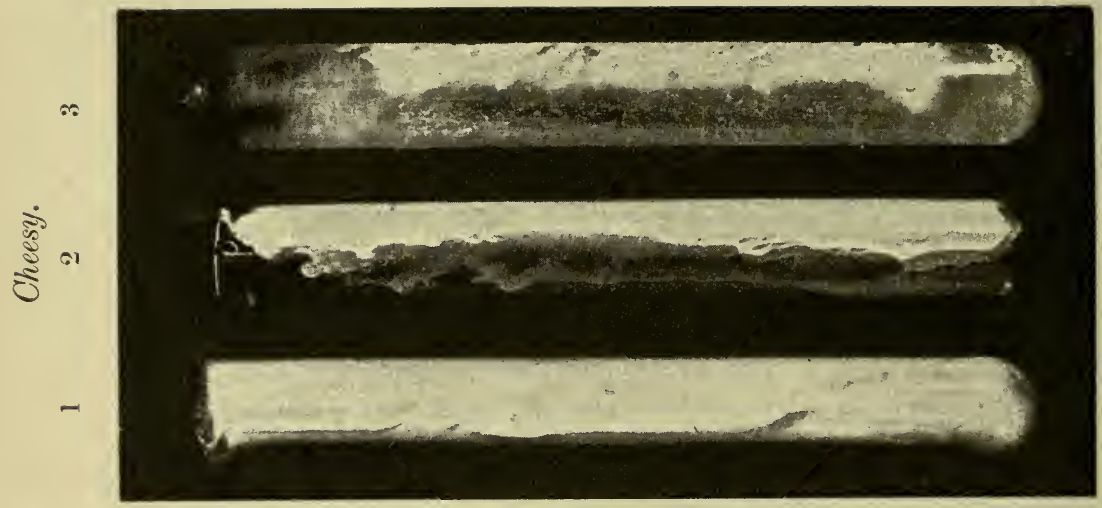

A

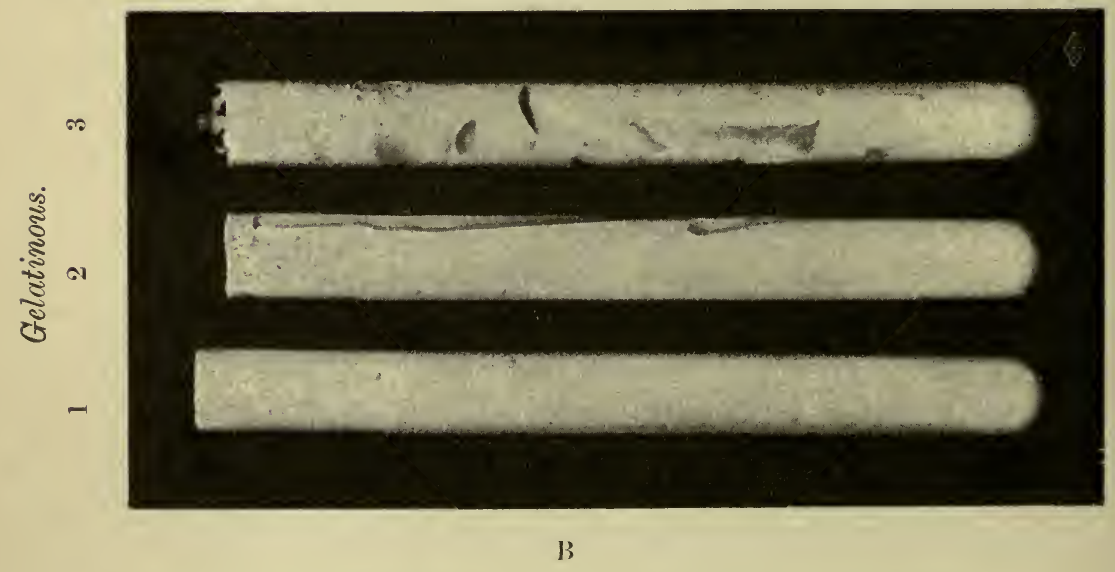

[After pane 1:6. 


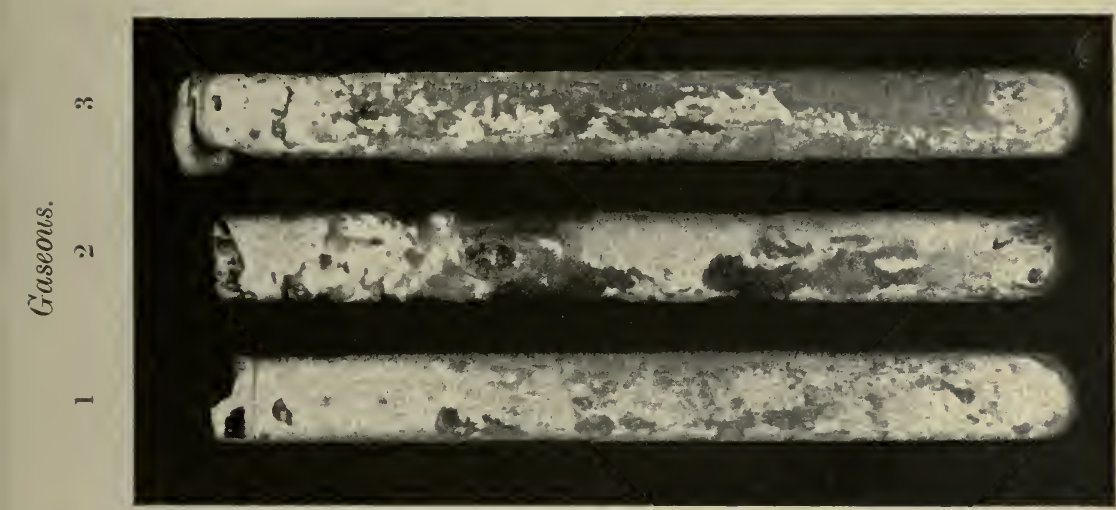

C

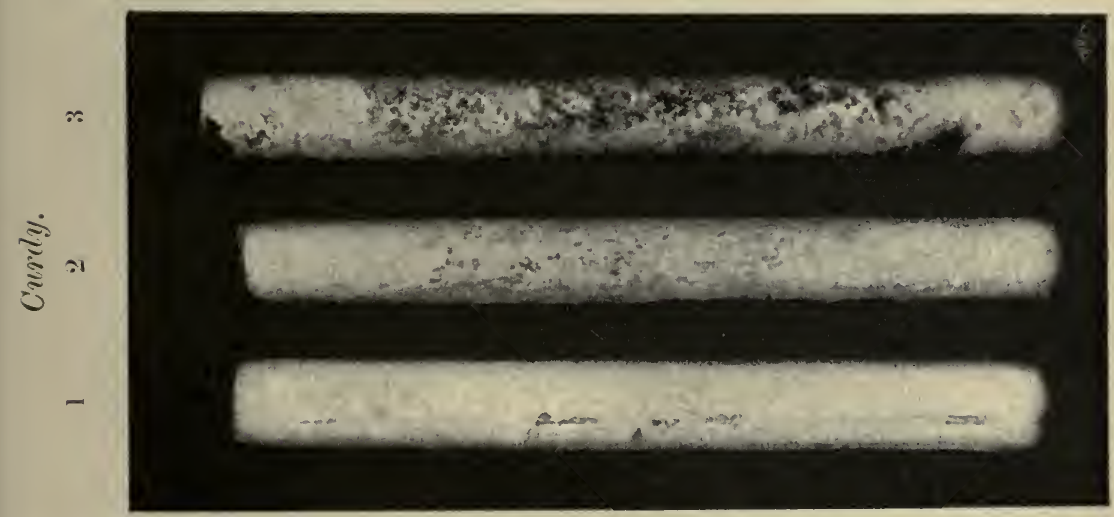



Finally, if the smell is unpleasant and there are numerous gas bubbles present, then the milk is absolutely unfit for human consumption or for dairy purposes. Often a clear layer is to be seen below the cream, whilst the rest of the mass is evenly coagulated. This is caused by the so-called peptonising bacteria (principally hay bacteria), and is an indication that these unwelcome guests are present in large numbers in the milk.

A. Peter, who has thoroughly investigated the fermentation test, gives five types of fermentation, each divided into three stages. He allows the fermentation to take place for longer than twelve hours, namely, fifteen to twenty-four hours at $38^{\circ}-40^{\circ}$. A synopsis of the different appearances which milk may have after fermentation is given on the opposite page. The table is based upon the original photographs taken by Peter, and those portions which appear in ordinary type are to be regarded as normal manifestations of fermentation, and not to be due to impure milk. The sections printed in italics are doubtful manifestations, whilst those in capitals indicate a milk which is dangerous to consume or to use for making cheese.

When use is made of Peter's table it is possible to get good comparative results, and use can be made of the contractions used in the table when noting down the result.

The fermentation test is used with much success in various countries, particularly in Switzerland, for testing the milk delivered to cheese factories to ascertain its suitability for making into cheese. Its use could be extended to analytical laboratories with advantage, for from this test a good notion is obtained of the bacterial condition of the milk. Attention must, however, be once more drawn to the necessity of thoroughly cleaning, preferably also sterilising the test-tubes, for without such precautions the results are worthless.

\section{'The Reductase Test.}

This test is based upon the fact first noticed by Duclaux ${ }^{1}$ that ordinary normal cow's milk has the power of converting

1 Le Lait, études chimiques et microbiologiques, Paris, 1887. 
certain colouring matters, e.g. indigo-carmine, into the corresponding leuco-compound by reduction. He also showed that this property of milk depends upon the micro-organisms which it contains.

Winter Blyth ${ }^{1}$ proved that the same power of reduction is exercised upon litmus, but the application of it as a means of judging the quality of the milk is due to Neisser and Wechsberg, ${ }^{2}$ who proposed the use of methylene blue solution. Afterwards H. Smidt, ${ }^{3}$ P. Th. Miuller, ${ }^{4}$ and the author, ${ }^{5}$ worked further "at this method, and showed that there is really a distinct parallel between the number of organisms in the milk and the time required, under certain conditions, for a solution of methylene blue to be decolorised.

Fresh milk obtained in the usual way requires ten to twelve hours or more for the reduction to be accomplished, whilst a milk that has been badly handled and is very rich in bacteria brings about the decolorisation in a few minutes.

The test is extraordinarily easy to carry out, and the solution of methylene blue which is used is prepared in the following manner :

A few grams of methylene blue in powder (the zinc chloride double salt should be used) are digested with about 20 c.c. of alcohol for half an hour at ordinary temperature, and then 5 c.c. of this saturated solution diluted with 195 c.c. distilled water. One c.c. of this solution is then mixed with 10 c.c. of the milk which is to be examined, and a couple of c.c. of paraffin oil floated over it to keep out air, and the whole warmed at $45^{\circ}-50^{\circ}$ in a water-bath. If the milk becomes colourless within an hour it must be regarded as very bad from a hygienic standpoint, and it is absolutely unsafe for the feeding of young children. When the decolorisation takes place within three hours, the milk may be looked upon as of second quality, whereas if the colour persists for more than three hours the milk is good.

It must not be concluded that the fermentation and reductase

1 Analyst, 1901, Vol. XXVI, p. 148.

2 Münchener Med. Wochenschrift, 1900, No. 37.

"Hygienische Rundschau, 1904, Vol. XIV, p. 1,137.

+ Archiv für Hygiene, 1906, Vol. LVI, p. 108.

5 Zeitschrift für Untersuchung der Nahrung.s- und Genussmittel, 1908, Vol. XV, p. 385. 
tests go hand in hand, and that the former can be replaced by the latter, which takes so much less time. The reductase test gives approximately the relative number of bacteria in the milk, whilst the fermentation test shows to what group the predominant bacteria belong, and whether they are useful or injurious. Where a simple and reliable test which will quickly show whether a milk is fresh and has been drawn with due regard to cleanliness, or whether it is old and dirty, is required, the reductase test can be thoroughly recommended. ${ }^{1}$

1 The Catalase test has not been included, because at the present time its general applicability has not yet been suffieiently investigated. 


\section{PART II-BUTTER}

\section{INTRODUCTORY.}

WHEN the majority of the fat is removed from milk by skimming or separating, the latter method now being the most usual, and the resulting cream subjected to powerful mechanical treatment, as in churning, the milk fat passes from its fluid, under-cooled state into a solid substance. The fatglobules are thereby formed into grains which are visible to the naked eye, and in size are about as big as the head of a pin. By washing and working the mass of impure butter, the greater part of the butter-milk is got from it, and the substance known as butter is left.

Butter can be made from either swect or sour cream. In the first case, the cream, directly after separating, is taken and made into butter, whilst in the second case the cream is allowed to sour, either by adding sour butter-milk or by means of a pure culture of lactic acid bacteria. Then the cream is left at the temperature of the room for about eighteen hours to allow of lactic acid fermentation taking place and the proper degree of ripening being reached, after which the cream is churned. Both kinds of butter can be made from pasteurised cream, and in this way a butter with better keeping qualities is obtained.

Generally a larger or a smaller quantity of salt is worked into the butter, the amount varying between 1 per cent. and 5 per cent., according to the length of time it is desired to keep it. In certain districts, however, butter is not salted at all.

A good sample of normal butter has the appearance of a more or less yellow, soft, and plastic mass with a dull surface. The plasticity of the butter, which differs from that of any other fat, is due partly to the variety of glycerides present in the butter and their relations to one another. Butter ought to have a fresh, aromatic smell and taste, and the brine should be perfectly clear, like water. 
When butter is kept, it undergoes a decomposition which makes it useless as food. This rancidity of the butter, as it is called, is due to the splitting up of the fat by certain microorganisms into glycerine and free fatty acids. Butter also sometimes acquires a tallowy flavour, which is the result of an oxidation process influenced by air and light.

The specific gravity of slightly salt butter made from sour cream is on an average 0.9625 at $15^{\circ} \mathrm{C}$., according to V. Storch's investigations. ${ }^{1} \quad$ Fleischmann ${ }^{2}$ gives the average specific gravity of unsalted butter as 0.9437 at $15^{\circ} \mathrm{C}$. The melting point and point of solidification of butter lie very near to the corresponding numbers obtained for pure butter fat. Prof. V. Storch, of Copenhagen, has studied the inner construction of butter very completely, and through his investigations an insight has been gained into its remarkably peculiar structure.

Butter does not consist of fat alone, but contains other substances which come from the milk. In round figures, it may be said that butter is composed of 84 per cent. fat and 16 per cent. of other substances, of which about 13 per cent. is water. The liquid present in butter which contains small quantities of other milk constituents, such as albumin, milk-sugar, and salts (also some added common salt), is called brine. It is this clear liquid which is to be seen in the form of small, clear drops on the freshly-cut surface of butter. Storch has shown, with the help of the microscope, that brine is present in butter in the form of an immense number of minute drops, the number and size of which vary considerably, but are in very close connection with the appearance and consistency of the butter. The number of these small drops of liquid in each cubic millimetre in butter varies from 2.5 to 13 millions, and, as regards size, the majority of the drops do not reach $0.01 \mathrm{~mm}$. in diameter, and only a small number exceed $0.05 \mathrm{~mm}$. Occasionally single drops of much larger diameter are to be found. Butter may therefore be regarded as an emulsion of brine in butter-fat.

To investigate the construction of butter, a small piece about the size of a pin-head is taken, and carefully spread out by pressing it between the slide and cover-glass. The microscopic

${ }^{1} 36^{\text {te }}$ Beretning fra den $\mathrm{Kgl}$. Vet- og Landbohöjskoles Lab. for landökonom. Forsög, p. 121.

${ }_{2}^{2}$ Lehrbuch der Milchwirtschaft. Fourth Edition. Leipsic, 1907, p. 266. 


\section{I32 MILK AND DAIRY PRODUCTS PART}

view is particularly well seen if, following Storch's example, the dark-field illumination is used. This is obtained by placing a small disc below the condenser of the microscope, and so cutting off the central rays, so that the preparation is only illuminated by the peripheral rays. A diaphragm is screwed into the objective. The drops of liquid now appear as small, highly refractive globules against a completely dark background of butter fat. ${ }^{1}$

Storch has shown that butter which has a dull, opaque appearance contains a very large number of extremely finely

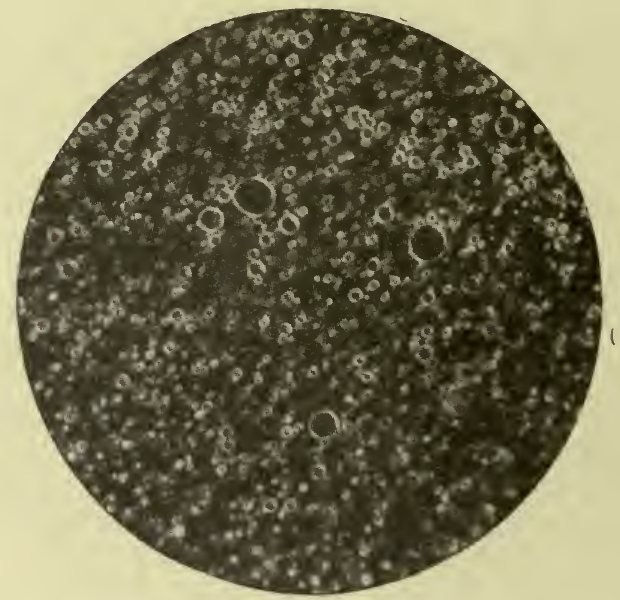

FIG. 54.-1:400. Barthel photo. Microphotograph of "Thick" Butter.

divided drops of liquid (see Fig. 54) whereas clear, bright butter (Fig. 55) has within it a much smaller number of these minute drops, the size of which, however, is larger than in the first case.

Butter on the surface of which drops of brine form on keeping contains not only these small, isolated drops, but also very large ones, often of irregular shape, and they are formed by the running together of the small drops. Although it appears from microphotographs as though the butter fat forms a homogeneous mass, this is not really the case. Storch has proved that the fat globules of the milk retain their original shape even after being churned into butter. In order to see the outline of

1 Figs. 54 and 55 show such dark-field photographs. 
the single globules of fat in the butter, a very thin preparation should be made, and the outermost edge examined under the microscope, preferably with a dark-field illumination. Even then it is very difficult to distinguish the separate fat globules.

Whilst the appearance and consistency of the butter are due principally to the number and size of the minute drops of liquid which it contains, there are still other factor's that contribute to these. For example, certain foods, insufficient cooling, \&c., can causo the butter to be soft and greasy, whilst the mechanical

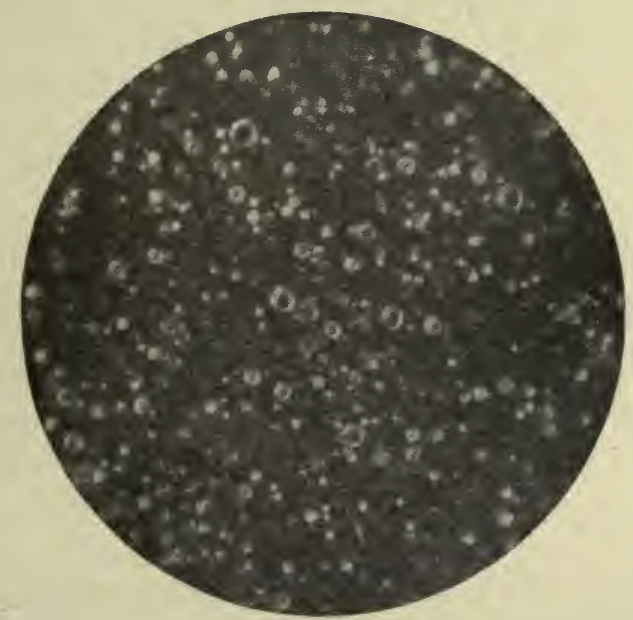

FIG. 55.-1 : 400. Barthel photo. Microphotograph_of "Clear" Butter.

processes of churning and working also exercise a considerable influence.

The chemical composition of butter is variable according to whether it is made from sweet or sour cream, and whether it is salted or not. According to Fleischmann, the chief sorts of butter have the following average composition:

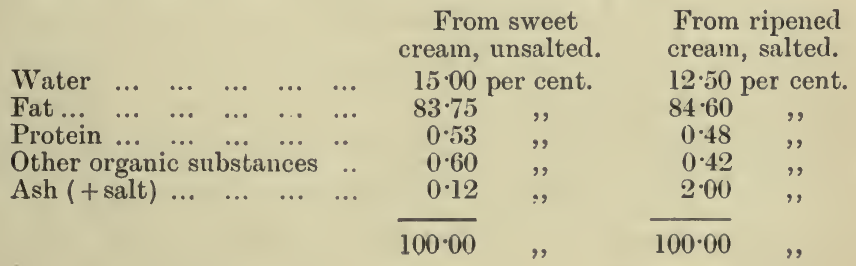


N. Engstrom ${ }^{1}$ gives the following analyses as typical of the average composition of salted butter made from ripened and fresh cream respectively:

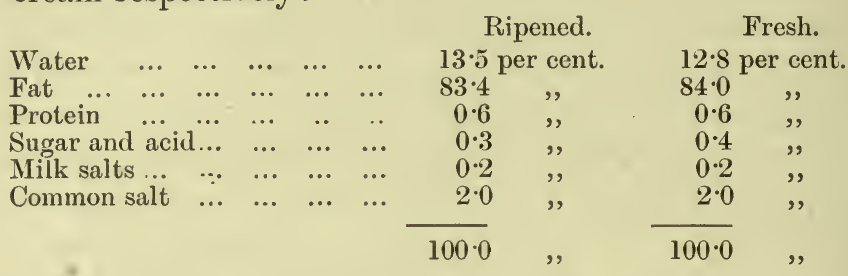

The variations in the different sorts of butter can, of course, be very considerable. Good butter ought not to contain less than 82 per cent. fat, and not more than 15 per cent. water, nor should the other components exceed 2 per cent., apart from the salt which has been added.

\section{Examination of Butter.}

\section{A. Sampling.}

There is no difficulty in obtaining a fair sample of butter for analysis, provided that small portions are taken from different parts of the whole mass. The composition varies considerably in the different parts, and so the sample cannot be taken from one place alone. Special borers are used for taking the samples. They vary in size, and have the form of a segment of a circle when seen in cross section. As will be seen in the figure below, a wooden handle is fixed in the upper part of the borer.

When taking a sample, the borer is pushed deep into the mass of butter, then turned round once and withdrawn. A cylindrical piece of butter then remains tightly attached to the borer. The sample is put into a wide-mouthed bottle with ground stopper, and other samples are taken from different places and added to the first; the main sample must weigh not less than 150-200 g. It is necessary to keep samples of butter in tightly closed vessels, otherwise some of the contained water evaporates.

Samples of butter should never be kept for a long time, otherwise they undergo change and become tallowy, rancid, \&c. A cool, dark place is the best in which to keep them.

1 Handbok i Mejerihushålning (Landtbrukets Bok), Stockholm, 1902, p. 432. 
If the sample of butter is to be examined chemically, it should be melted by placing the bottle in water at $40^{\circ} \mathrm{C}$. A white layer, composed of the water from the butter along with dissolved salts and casein, collects at the bottom, and above it the butter fat floats. On shaking vigorously, the under layer can be again incorporated with the fat, and this must be done immediately before a sample is taken. The condition of emulsion of the brine is not altered by the above-mentioned separation of the non-fatty substances.

If a small portion of the white deposit is examined under the microscope, it is seen that the brine is still in the form of enormous quantities of minute drops of liquid, completely separated from one another. The melting of the butter has therefore not caused the drops of brine to run together.

When butter is melted at a higher temperature, e.g., $50^{\circ}-60^{\circ}$ C., the aqueous layer separates so quickly that it is difficult to take a sample after shaking, for the mixture does not remain homogeneous for suffici-
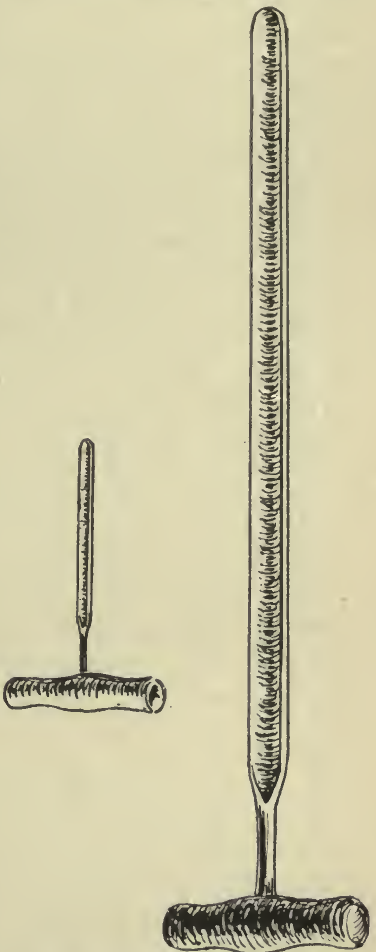

Fig. 56. - A Large and a Small Butter Sampler. ently long.

When butter is melted and the fat separated from the brine, it gives the so-called "butter lard," which is characterised by keeping good for a long time. The examination of it is carried out in the same way as for ordinary butter.

\section{B. The Chemical Analysis.}

\section{Determination of the Water.}

In practice a determination of the percentage of water in butter is, next to the proof of adulteration, the most important factor, for from it a simple, convenient, and, for most purposes, 


\section{I36 MILK AND DAIRY PRODUCTS PART}

sufficiently accurate estimate of the amount of fat present can be obtained. Apart from the fat and water, the other components of butter remain remarkably constant for the same sort of butter. For salted butter made from ripened cream the amount averages about 3 per cent. If the percentage of water in a butter were found to be 13.50 , then the fat would be about 83.5 per cent. if the butter were salted and made from ripened cream.

In many countries (Germany, England, Switzerland, Holland, and Belgium), laws prescribing the maximum of water and the minimum of fat that is allowable in butter offered for sale are in operation. In Germany not more than 18 per cent. of water is allowed in salted butter, and 16 per cent. in unsalted. The amount of fat must not be less than 80 per cent. In England the standard for water is fixed at 16 per cent.

The percentage of water in butter is easily and conveniently determined in the following manner.

About 15 g. pumice stone, which have been ignited and broken into pieces the size of a pea, are put into a flat porcelain dish, with vertical sides, measuring about $6 \mathrm{~cm}$. in diameter. The dish and its contents are dried in the drying oven for half an hour at $100^{\circ} \mathrm{C}$., allowed to cool, and then weighed.

In the meantime, the sample of butter has been melted in water at $40^{\circ} \mathrm{C}$., well shaken, and about $5 \mathrm{~g}$. poured over the pumice stone in the dish. The whole must be quickly weighed, then placed in a drying oven for one hour at $100^{\circ} \mathrm{C}$., after which the weighing is repeated and the dish returned to the oven. The weighings are made every half-hour until the weight is constant. Generally about two hours are required when working in the above manner.

The butter must not be dried for longer than three hours, for long-continued heating causes the fat gradually to oxidise, and in consequence the weight of the butter increases.

A quite constant weight cannot be got, but where the samples are dried for the same length of time at the same temperature good usable results are to be got. 'When it is a question of rapidity rather than of great accuracy, the water may be estimated by the following method, as described by Droop Richmond. ${ }^{1}$ 
About $10 \mathrm{~g}$. butter are weighed out, together with a short glass rod, into a porcelain dish, which is then heated over a small flame, the butter being kept constantly stirred and the flame regulated so that no fat spirts out. In this way all the water is evaporated, and a point is reached where the boiling stops suddenly and a fine foam forms on the surface, whilst the casein at the bottom becomes a light brown colour. $\Lambda$ s the water has then all been driven off, the flame is withdrawn. Even though the heating should be continued a little too long, it does not make much difference. When the dish is cold it is weighed again, and so the loss of water is obtained.

This method, which appears to be quite crude, really gives surprisingly accurate results, and the author has found that the mean difference between this method and the ordinary method with pumice stone is on an average only +0.17 per cent.

\section{Determination of the Fat.}

(a) Scientific Methods.

\section{The Extraction Method.}

If the fat of the butter is to be determined by the extraction method, the residue left after the determination of the water is used. With the help of a platinum spatula, the pumice stone and the dried butter are transferred to one of the fat-free cartridges made by Schleicher and Schiull, and this put into a Soxhlet extraction apparatus. Afterwards the dish is washed out with small quantities of ether, which are poured on to the cartridge. When all the fat has been washed from the basin, a fairly thick layer of previously extracted cotton wool is placed over the cartridge, which also rests upon a layer of similar cotton wool 2-3 cm. thick. These precautions are necessary, for otherwise some of the fine particles of pumice stone would be carried over with the ether. The extraction with pure ether must proceed for at least 12 hours, after which the ether is evaporated off and the fat dried and weighed. This is done in exactly the same manner as described in the extraction of fat in milk (p. 49). 


\section{Röse-Gottlieb Method.}

This method, which was originally designed for the estimation of fat in milk, and is described on p. 52, can be used with advantage also for the determination of fat in butter.

According to A. Hesse, ${ }^{1}$ about 2 g. of butter are weighed out into a $3 \mathrm{~cm}$. long, half-cylindrical glass tube, or simply wrapped in a piece of stiff fat-free paper of the same form. The tube or paper and the contained fat are then introduced into a Gottlieb cylinder, and hot water added until the $10 \mathrm{~cm}$. mark is reached. If the butter does not melt, the cylinder is placed in warm water until it does. Then 1 c.c. of ammonia and 10 c.c. of 95 per cent. alcohol are added exactly as in the estimation of milk fat. If the mixture is still warm, the cylinder is cooled down in cold water so that the ether which is to be added will not evaporate so quickly. The cooling must not, however, be carried too far, otherwise the butter will become solid again. Twenty-five c.c. of ether are then added, the contents of the cylinder mixed by repeatedly inverting it, and afterwards 25 c.c. of petroleum ether are added and the mixing repeated.

After the different layers have separated quite sharply from one another, the clear ether-fat solution is siphoned off in the usual way, the lower opaque layer not being disturbed. Then 50 c.c. of ether are poured into the cylinder and at once siphoned off without being mixed with the other liquid. Finally, the residual liquid is shaken with a mixture of 25 c.c. ether and 25 c.c. petroleum ether, and, after settling, the ethereal layer is drawn off. The three lots of ether are naturally all placed in the same tared flask, which is weighed again after the ether has been evaporated and the fat dried.

These repeated extractions with ether and petroleum ether are necessary if exact results are to be got. If the above directions are carefully followed, the results will be found to be in very close agreement with those obtained from the extraction method. Thus the Röse-Gottlieb method is easier and more convenient, and also considerably quicker than the extraction method. 


\section{Indirect Determination of Fat.}

By determining the percentage of water and of non-fat in butter, and then subtracting these numbers from 100 , the percentage of fat can be obtained. The same degree of accuracy as with the direct estimation is not, however, to be reached, for it can easily happen that the analytical errors in the water and non-fat determinations are added together, and so a serious difference results.

\section{(b) Practical Methods.}

\section{Bernstein's Method.}

Of the numerous practical methods for determining the amount of fat in butter, mention will only be made of that due to Alex. Bernstein, ${ }^{1}$ for it is the only one that gives applicable results.

The necessary apparatus ${ }^{2}$ is shown in Fig. 57 , and consists of a glass cylinder, $a$, and a tube, $b$, in which the estimation is made. The glass cylinder, $\alpha$, is closed by a wooden stopper, $c$, into which the tube, $b$, also fits. Rubber stoppers close each end of tube, $b$. A small syringe for measuring out glycerine also belongs to the apparatus.

To carry out the test, the tube, $b$, which must be quite clean and dry, is closed by the two rubber stoppers; the smaller of these must be so adjusted as to reach to the black mark on the tube. The apparatus then has the appearance shown in the sketch.

After the larger stopper has been removed, $10 \mathrm{~g}$. of butter are weighed out upon a strip of parchment paper $(7 \times 4 \mathrm{~cm}$.), an accurate balance being used. Two c.c. of glycerine $\left(28^{\circ}\right.$ Beaumé) are measured into the tube, and

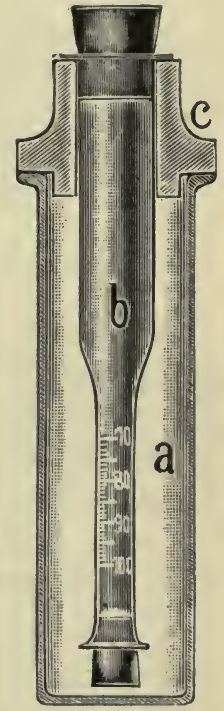

Frg. 57.-Apparatus for Bernstein's Method. afterwards the paper containing the butter is slipped in. Enough pure benzine (petroleum ether) is added

1 Milch-Zeitung, 1905, No. 18.

2 To be had from Alex. Bernstein, 9 Magdeburgerstrasse, Berlin. 
to fill the tube to the upper mark. It is better to take too much rather than too little benzine. The tube, $b$, is now taken from the cylinder, $a$, the latter filled with water at $55^{\circ}$ to within $4 \mathrm{~cm}$. of the upper edge, and the tube replaced. After the butter has melted, the protein is seen to have separated in a very voluminous form, the paper is withdrawn, and the stopper put into place. The tube, $b$, along with the wooden stopper, $c$, is lifted from the cylinder and the rubber stopper pressed in with the flat of the hand. The apparatus is then inverted, so that the large stopper is below, and the contents thoroughly mixed by vigorous shaking. Afterwards the tube, $b$, is returned to $a$, and the apparatus left to stand for four hours. During this time the non-fatty substances separate completely, leaving a sharp dividing line below the protein layer and the solution of fat. When the temperature of the water in $a$ has reached that of the room, and not before, the percentage of fat is read off on the graduated scale.

It has been shown by A. Hesse ${ }^{1}$ and by the author that this method gives results which only in exceptional cases differ by 1 per cent. from those obtained by gravimetric analysis. For all practical purposes such results are sufficiently accurate.

\section{Determination of the Anhydrous Solids not Fat.}

In addition to fat and water, there are found in butter small quantities of protein, milk-sugar and other nitrogen-free substances (lactic acid), and also mineral matter. When the butter has been salted there is also common salt.

An estimation of the total amount of these substances is made in the following manner: Five to $10 \mathrm{~g}$. of butter (with . unsalted butter 10-15 g.) are weighed out into a small glass beaker, or better into a glass dish with spout, of about $10 \mathrm{~cm}$. diameter. The butter is then melted at a low temperature, some petroleum ether added, and the mixture filtered through a Swedish filter that has previously been dried and weighed. As far as possible the sediment should be left in the dish. Further treatment with petroleum ether and subsequent decantation through the filter free the sediment from the greater part of the fat. The mass should not, however, 
be entirely freed from fat, otherwise when it dries it is difficult to remove from the glass. The dish containing the solids not fat is now dried for a couple of hours at $100^{\circ} \mathrm{C}$., after which the residue is brought on to the filter which was previously used, and by washing repeatedly with petroleum ether it is freed completely from fat. When all fat has been removed a drop of the filtrate leaves no residue when evaporated on a watch-glass. The filter containing the solids not fat can then be dried and weighed in the usual way.

\section{Determination of Protein.}

This cannot be done successfully by the Kjeldahl method unless most of the fat bas been removed, for otherwise an objectionable amount of frothing takes place. The best way is to treat the butter as in the determination of the solids not fat, only a larger quantity of butter should be taken, and the filter need not be washed entirely free from fat:

The filter and the solids not fat are then treated in the usual way, and the amount of nitrogen which is obtained is multiplied by 6.37 . The percentage of nitrogen in the filter paper can be determined by analysing a few of the same kind and then deducting that which is due to the filter from the above result.

\section{Estimation of Common Salt.}

To estimate the total amount of ash, the butter is treated as for the solids not fat, the filter and the residue then carefully ignited in a platinum dish, and the weight of the filter ash deducted. The weight which is thus obtained is the total ash. The ash is then treated with water, filtered, and the sodium chloride determined by titration with $\mathrm{N} / 10 \mathrm{AgNO}_{3}$, potassium chromate being used as an indicator.

By subtracting the amount of sodium chloride so obtained from the total ash, there is left the quantity of milk salts (crude ash) present in the butter.

\section{Vi. Proof of Pasteurisation.}

'To find out whether the cream used in making a butter has been pasteurised at a temperature of $80^{\circ} \mathrm{C}$. or more Storch's 
reaction (p. 98) can be used with advantage and in the following manner.

About $25 \mathrm{~g}$. of butter are placed in a suitable beaker and there melted by putting the beaker into warm water of a temperature not higher than $60^{\circ} \mathrm{C}$. The clear butter fat is then drawn off and the white sediment mixed with an equal quantity of water. The resulting liquid is then placed in a test-tube and one drop of hydrogen peroxide and two drops of paraphenylenediamine solution added. If a blue coloration is given, then the cream from which the butter was made had not been heated to $80^{\circ} \mathrm{C}$.; if, on the contrary, there is no coloration, then the heating must have exceeded this temperature. This method usually fails if the butter is more than fourteen days old.

\section{Examination of the Butter Fat.}

A. Physical Tests.

\section{Determination of the Specific Gravity of Butter Fat (at $100^{\circ}$ C.).}

The sample of butter is melted at about $60^{\circ} \mathrm{C}$. and the clear fat poured off from the sediment through a dry filter, care being taken that none of the milky sediment enters. This clear filtered fat is pure butter fat.

E. König recommends that a thin-walled test-tube should be filled with the fat and suspended almost up to the top in a water-bath containing boiling water. By means of a specially constructed hydrometer, ${ }^{1}$ having a scale of $0.845-0.870$, the specific gravity is then determined. In general this lies between 0.865 and 0.868 at $100^{\circ} \mathrm{C}$. if the fat is pure.

\section{Determination of the Melting Point and the Point of Solidification.}

(a) The Melting Point. ${ }^{2}$

A piece of glass tubing with an internal diameter of about $3 \mathrm{~mm}$. is drawn out in such a way that the change from the wide tube to the capillary is quite sharp. The drawn-out

1 To be obtained from C. Gerhardt, Bonn.

2 Bensemann, Rep. der anal. Chem., Vol. IV, p. 165, and Vol. VI, p. 202. 
portion (the capillary tube) is cut off about $5 \mathrm{~cm}$. below the point where the tube was drawn out. A couple of drops of clear filtered butter fat are now put into the wider point of the tube, and by manipulation are made to assume the position shown in Fig. 58 a. After the butter has become quite solid, which is done by placing the tube on ice for a couple of hours, the latter is attached by means of a thin rubber band to the thermometer; the drop of fat being placed on a level with the bulb. The thermometer and the tube are now fastened to a retort stand and sunk in a small beaker of water, which is heated slowly over a small flame, the water being stirred constantly.

When the fat begins to appear translucent the temperature is noted. This is the beginning of the melting, and the drop of fat then commences to run and take up the position shown in Fig. 58 b. When the fat is quite transparent the temperature is noted, and this point is known as the end of the melting.

The melting point of pure butter fat, which has no definite relation to the Reichert-Meissl Value, Hehner's Value, or Hübl's Iodine Number (see below), varies between $28^{\circ}$ and $34: 7^{\circ} \mathrm{C}$.

\section{(b) The Point of Solidification.}

To determine this a test-tube is filled to the height of 3 or $4 \mathrm{~cm}$. with clear melted

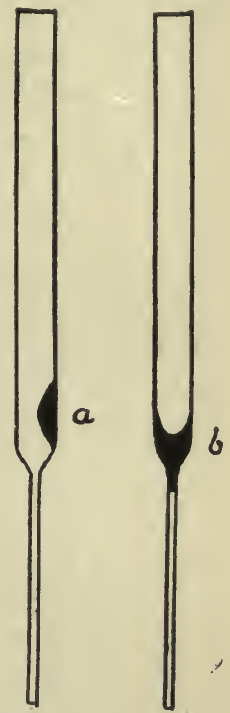

FIG. 58.-Melting Point Tube.

filtered butter fat, and a thermometer sunk into the fat so that the bulb is entirely below the surface. The testtube is then attached to a retort stand and sunk in a beaker of water having a temperature of $40-50^{\circ}$ C., and the water allowed to cool gradually. At first the temperature sinks regularly, and after a time becomes constant. After it has remained constant for a while the mercury begins to fall rapidly. The temperature shown by the thermometer whilst the column of mercury is at rest is the point of solidification. As long as the solidification of the fat is taking place the 
temperature is constant, and for butter fat lies between $19^{\circ}$ and $23^{\circ} \mathrm{C}$.

\section{Determination of the Critical Temperature of Solution in Alcohol (Crismer's Method).}

Crismer ${ }^{1}$ has proposed, as a means of testing butter fat, to determine the critical temperature of solution in alcohol. By the critical temperature is understood the temperature at which a mixture of one part butter fat forms or ceases to form a clear solution with three parts by volume of alcohol.

To carry out this determination test-tubes about $10 \mathrm{~mm}$. in diameter and $100-120 \mathrm{~mm}$. long are used, and they are provided with two marks, the upper one denoting double the volume of the lower. A sensitive thermometer with a very small bulb and opal glass scale is used. The alcohol employed must have a specific gravity of exactly 0.7967 at $15.5^{\circ} \mathrm{C}$. If the specific gravity is different, it must be corrected by adding or deducting $0 \cdot 186^{\circ}$ for each 0.0001 above or below the specific gravity given above (see also Table IX in Appendix).

The test is carried out by filling one of the test-tubes to the lower mark with filtered fat, and then with alcohol to the upper mark. With the aid of a cork the thermometer is fitted into the mouth of the tube so that the bulb comes into the middle of the liquid. Afterwards the tube is gently warmed and shaken until a clear transparent solution is obtained. The flame is then withdrawn and the shaking repeated until the liquid begins to get cloudy again, and the temperature is then noted.

A greater or less degree of acidity affects the result, and causes it to be lower. To prevent this the butter must either be neutralised by treating it with a 10 per cent. solution of potassium carbonate, and then washed with warm water until quite clear, or, as is more usual, the following correction is applied :

The degree of acidity of the butter is estimated by the number of cubic centimetres of $N / 20$ potash which are required to neutralise 2 c.c. of butter fat dissolved in 20 c.c. of absolute

${ }^{1}$ Bull. Assoc. Belge des Chimistes, March, 1897 ; Vademecum du Chimiste, Paris, 1903, p. 114. 
alcohol, phenolphthalein being used as indicator. The number of cubic centimetres of $N / 20$ potash is added to the critical temperature which has been found, and the sum expresses the real critical temperature. As it is very tedious and difficult to determine the specific gravity of the alcohol before each test, Crismer ${ }^{1}$ has proposed to use a standard petroleum, by which means first the specific gravity of commercial absolute alcohol is determined, and afterwards the critical temperature of the mixture of petroleum and alcohol.

The method of constructing a curve which can be used for obtaining the required correction for alcohol of any strength can be seen in Crismer and Vandam's original paper. ${ }^{2}$

The critical temperature of solution of butter-fat in alcohol lies generally between $53^{\circ}$ and $57^{\circ} \mathrm{C}$; ; in exceptional cases the upper limit may reach $59^{\circ} \mathrm{C}$.

\section{Determination of the Refractive Index.}

The refractive index is generally determined with a Wollny butter refractometer made by Zeiss, of Jena. With slight modifications, this instrument is the same as the milk refractometer described on p. 40.

The graduation of the scale of the butter refractometer is from -5 to +105 , corresponding to refractive indices of 1.4179-1.4922. It is therefore a continuation of the scale of the milk refractometer.

The method of using the butter refractometer is exactly the same as described for the milk refractometer, a few drops of clear filtered butter fat being placed between the prisms. A refractometer and an apparatus for supplying water at a constant temperature are all that is required, and the determination is generally made at $40^{\circ} \mathrm{C}$. The coloured critical line between the illuminated and the dark fields is rendered colourless in the butter refractometer by a special arrangement of the upper prism, so that with normal butter the critical line appears sharp and colourless.

To make a determination, the faces of the prisms must be cleaned with the greatest care; a soft, clean piece of fine linen,

1 Bulletin de la Société chimique de Belgique, Vol. XVIII, Jan., 1904.

2 Ibid, Vol. XX, 1906 ; Third International Dairy Congress, Section 1, No. 16. 


\section{I46 MILK AND DAIRY PRODUCTS PART}

moistened with a little alcohol and ether, is recommended. A few drops of clear filtered butter fat are brought on to the upper face of the lower movable prism. It is best to do this whilst holding the refractometer in the left hand, and inclining it until the face of the prism in question is horizontal. The butter need not necessarily be filtered, and the requisite quantity can be got by dipping a well-rounded glass rod into the melted fat, taking care that none of the impurities floating on the surface are withdrawn.

When the prism box has been closed again the refractometer is replaced in its original position, and the refractive index can be read off as with the milk refractometer. Daylight or lamplight is used for illumination.

Before taking a reading it must be seen whether the space between the faces of the prisms is evenly filled with butter fat. To do this the image of the faces of the prism which lies about $1 \mathrm{~cm}$. before the ocular is examined with a magnifying glass or with the naked eye, the latter being held at a certain distance from the ocular. In this way air bubbles in the layer of fat are easily detected; they exercise a disturbing effect upon the sharpness of the critical line.

Before taking the reading of the scale a minute or two should be given for the butter fat and the prism to take the same temperature, for it is only when this has been reached that the critical line becomes clear and definite.

For every whole degree of temperature above or below that for which the refractometer is graduated a correction of 0.55 must be made, and this number added to or deducted from the refractometer reading.

According to Wollny, the limits for natural butter are $49.5-54.0$ divisions of the scale at $25^{\circ} \mathrm{C}$. The corresponding indices of refraction are $1 \cdot 4590-1 \cdot 4620$. At $40^{\circ} \mathrm{C}$. the limits are from 40.5 to 44.4 .

Along with each refractometer there is supplied a standard liquid for testing the accuracy of the instrument, and this must be done from time to time and the results compared with the figures given on the opposite page.

A fraction of a degree is easy to calculate $\left(0 \cdot 1^{\circ}=0.06\right.$ division of scale). Differences of one- or two-tenths of a division are of no importance, and they are generally the result of faulty 
determinations of temperature. When, however, the differences are greater than this the refractometer scale must be readjusted. For this purpose the newer butter refractometers are provided with a micrometer screw, which can also easily be attached to the older instruments. The arrangement of this micrometer screw is seen in Fig. 59 below.

Refractometer Numbers for the Standard Liquid.

\begin{tabular}{c|c||c|c|c|c}
\hline \multirow{2}{*}{ Temp. } & $\begin{array}{c}\text { Scale } \\
\text { divisions. }\end{array}$ & Temp. & $\begin{array}{c}\text { Scale } \\
\text { divisions. }\end{array}$ & Temp. & $\begin{array}{c}\text { Scale } \\
\text { divisions. }\end{array}$ \\
\cline { 2 - 3 } $25^{\circ}$ & $71 \cdot 2$ & $19^{\circ}$ & $74 \cdot 9$ & $13^{\circ}$ & $78 \cdot 6$ \\
24 & $71 \cdot 8$ & 18 & $75 \cdot 5$ & 12 & $79 \cdot 2$ \\
23 & $72 \cdot 4$ & 17 & $76 \cdot 1$ & 11 & $79 \cdot 8$ \\
22 & $73 \cdot 0$ & 16 & $76 \cdot 7$ & 10 & $80 \cdot 4$ \\
21 & $73 \cdot 6$ & 15 & $77 \cdot 3$ & 9 & $81 \cdot 0$ \\
20 & $74 \cdot 3$ & 14 & $77 \cdot 9$ & 8 & $81 \cdot 6$ \\
\hline
\end{tabular}

From the table given above it is seen what number of divisions ought to be given with the standard liquid at the temperature shown on the thermometer. Suppose this number to be $76 \cdot 7$. The nut, $G$, on the micrometer screw is loosened, and the nickel disc, $S$, turned until the critical line seen in the eye-piece falls exactly upon the whole division of the scale -in this case 76 . Then the small black cylinder, $F$, which is now loose, is turned until the

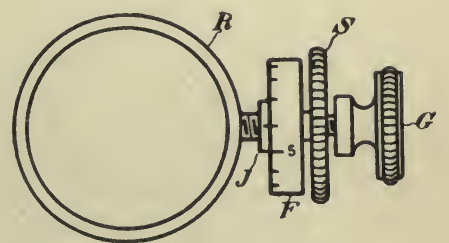

FIG. 59-The Micrometer Screw. pointer, $J$, shows the desired tenths of a degree-in this case 7. The loose nut, $G$, is then screwed tight, and the accuracy of the refractometer again tested.

Naturally, the refractometer can be used for all liquids the refractive index of which falls between 1.418 and 1.492 , and it also serves for substances which can be brought into a fluid state by warming or by solution.

The table given below serves to convert the scale divisions into refractive indices at $25^{\circ} \mathrm{C}$. In column $\Delta_{n}$ are shown the 


\section{I48 MILK AND DAIRY PRODUCTS PART}

differences for each scale division, expressed as units of the fourth decimal:

T'able of Refractive Indices.

\begin{tabular}{c|c|c|c|c|c||c|c|c}
\hline $\begin{array}{c}\text { Scale } \\
\text { divisions }\end{array}$ & $n_{\mathrm{D} \cdot}$ & $\Delta_{n} \cdot$ & $\begin{array}{c}\text { Scale } \\
\text { divisions }\end{array}$ & $n_{\mathrm{D} \cdot}$ & $\Delta_{n} \cdot$ & $\begin{array}{c}\text { Scale } \\
\text { divisions }\end{array}$ & $n_{\mathrm{D} \cdot}$ & $\Delta_{n} \cdot$ \\
\hline-5 & $1 \cdot 4179$ & $8 \cdot 2$ & 35 & $1 \cdot 4488$ & $7 \cdot 2$ & 75 & $1 \cdot 4723$ & $\mathbf{6 \cdot 2}$ \\
\hline 0 & $1 \cdot 4220$ & $8 \cdot 0$ & 40 & $1 \cdot 4524$ & $7 \cdot 0$ & 80 & $1 \cdot 4754$ & $5 \cdot 8$ \\
5 & $1 \cdot 4260$ & $8 \cdot 0$ & 45 & $1 \cdot 4559$ & $6 \cdot 8$ & 85 & $1 \cdot 4783$ & $5 \cdot 8$ \\
10 & $1 \cdot 4300$ & $7 \cdot 8$ & 50 & $1 \cdot 4593$ & $6 \cdot 6$ & 90 & $1 \cdot 4812$ & $5 \cdot 6$ \\
15 & $1 \cdot 4339$ & $7 \cdot 6$ & 55 & $1 \cdot 4626$ & $6 \cdot 6$ & 95 & $1 \cdot 4840$ & $5 \cdot 6$ \\
20 & $1 \cdot 4377$ & $7 \cdot 6$ & 60 & $1 \cdot 4659$ & $6 \cdot 4$ & 100 & $1 \cdot 4868$ & $5 \cdot 4$ \\
25 & $1 \cdot 4415$ & $7 \cdot 4$ & 65 & $1 \cdot 4691$ & $6 \cdot 4$ & 105 & $1 \cdot 4895$ & $5 \cdot 4$ \\
30 & $1 \cdot 4452$ & $7 \cdot 2$ & 70 & $1 \cdot 4723$ & $6 \cdot 2$ & & $1 \cdot 4922$ & \\
\hline
\end{tabular}

In many laboratories the Abbé refractometer is used (see Fig. 60 ), and this differs chiefly from the milk and butter refractometers in having a more comprehensive scale, one from $n_{\mathrm{D}}=1.3$ to $n_{\mathrm{D}}=1 \cdot 7$. This admits of more extended use, and also the refractive index is not read on the scale in the eye-piece, but on a sector, $S$ (Fig. 59), which is firmly united to the tube of the refractometer, and from which the refractive indices can be read off directly.

A more detailed description of the apparatus will not be given here, for the manufacturer (Carl Zeiss, Jena), supplies full particulars of each instrument when bought, and also the method of using it.

Abbe's refractometer can therefore be used for the determination of milk fat and milk serum as well as for the investigation of butter, \&c. The refractive indices can be read off on the sector, $S$, with an exactitude of about 0.0002 . The results, however, are hardly as exact as those obtained by the refractometer specially designed for milk and butter, and this is due to the greater distance covered by the scale in Abbé's refractometer.

\section{B. Chemical T'ests.}

\section{The Reichert-Meissl Number.}

The Reichert-Meissl Number is the amount of $N / 10$-alkali, expressed in cubic centimetres, which is required to neutralise the volatile and water-soluble fatty acids obtained from $5 \mathrm{~g}$. of butter fat under certain conditions. 
Reichert ${ }^{1}$ was the inventor of this method, and C. Meissl ${ }^{2}$ and R. Sendtner ${ }^{3}$ modified it. The manner of carrying out the determination is as follows:

Exactly $5 \mathrm{~g}$. of clear filtered butter fat, which has been well mixed, is weighed out into a round-bottomed flask of about

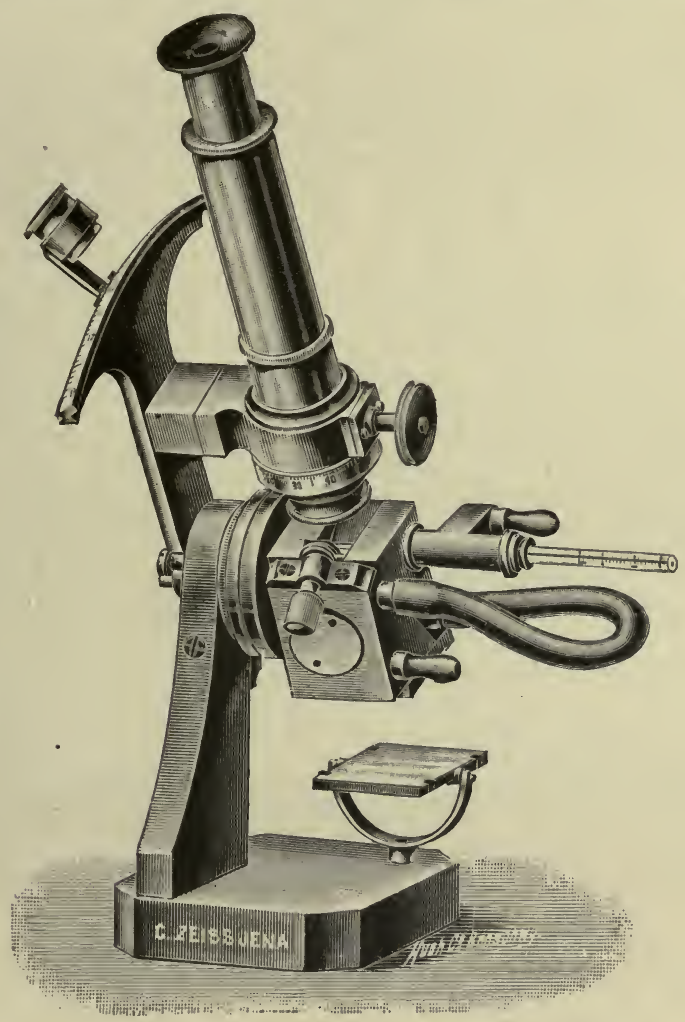

Fig. 60.-Abbé's Refractometer.

300 c.c. capacity, and the flask placed upon a boiling waterbath. When the fat has completely melted, 10 c.c. of alcoholic potash (20 g. caustic potash in 100 c.c. 70 per cent. alcohol) are added from a pipette (the liquid should not be blown out), and the whole well shaken. In consequence of the shaking and the warming on the water-bath the greater part of the alcohol

1 Zeitschrift für analyt. Chemie, 1879, Vol. XVIII, p. 68.

2 Dingler's Polyt. Journal, Vol. CCXXXIII p. 231.

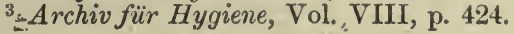


evaporates, and it is not long before frothing of the contents of the flask is noticed. In a few minutes (generally about seven) the saponification is finished. To get rid of the last traces of alcohol a current of air is blown in from the bellows and the flask moved from side to side. The blowing in of air is repeated each half-minute, after which the flask is again returned to the water-bath.

The alcohol may also be got rid of by fitting a rubber stopper with two glass tubes into the flask and then attaching one of the tubes to the water-pump so that a stream of air is drawn through, the Hask being kept in the water-bath. Air is drawn through the flask until all the soap has become solid and does not swell up when one of the glass tubes is closed and the pump makes a vacuum in the flask. The soap which has been freed from alcohol in the above manner is dissolved in 100 c.c. of distilled water, and ought to give a perfectly clear solution. If this is not so it is a sign of imperfect saponification, and the operation must be repeated. After the addition of the water several pieces of pumice stone, about the size of peas, are put into the flask and 40 c c. of dilute sulphuric acid ( 1 in 10$)$, and it is attached to a condenser of not less than $50 \mathrm{~cm}$. in length. Exactly 110 c.c. of liquid are distilled over into a flask provided with a mark at this level; the distillation should be completed within thirty minutes.

In this way the volatile soluble acids from the butter are distilled over, and along with them are also some volatile acids which are insoluble in water. To separate these the distillate is shaken up and poured through a dry filter, and 100 c.c. of the filtrate titrated with $N / 10$ caustic soda, a few drops of alcoholic phenolphthalein being used as indicator. By adding one-tenth to the number of cubic centimetres of caustic soda which have been used, the amount required for the whole distillate is obtained. The number of cubic centimetres of $N / 10$ caustic soda is known as the Reichert-Meissl number.

Leffmann and Beam ${ }^{1}$ have worked out a modification of the method, and at the present time this is used more extensively than the original. In carrying out this modification, $5 \mathrm{~g}$. of fat are weighed out as before in a 300 c.c. round-bottomed flask of Jena glass, and then 20 c.c. of pure glycerine and 2 c.c. of caustic

1 Chemiker-Zeitung, No. 62, p. 607. 
soda (100 g. in 100 c.c. water) added. The flask is then heated over a small flame, the contents being kept in movement for a few minutes. When the water has all evaporated the liquid ceases to boil and becomes quite clear.

The soap is dissolved in 90 c.c. of boiling water, a few pieces of pumice stone dropped into the flask, and then 50 c.c. of sulphuric acid ( 25 c.c. concentrated acid per litre) added. The caustic soda solution mentioned above ought to be neutralised by $30-35$ c.c. of this dilute sulphuric acid. After the addition of the acid the flask is connected at once with the condenser and 110 c.c. distilled over as usual, the time of distillation

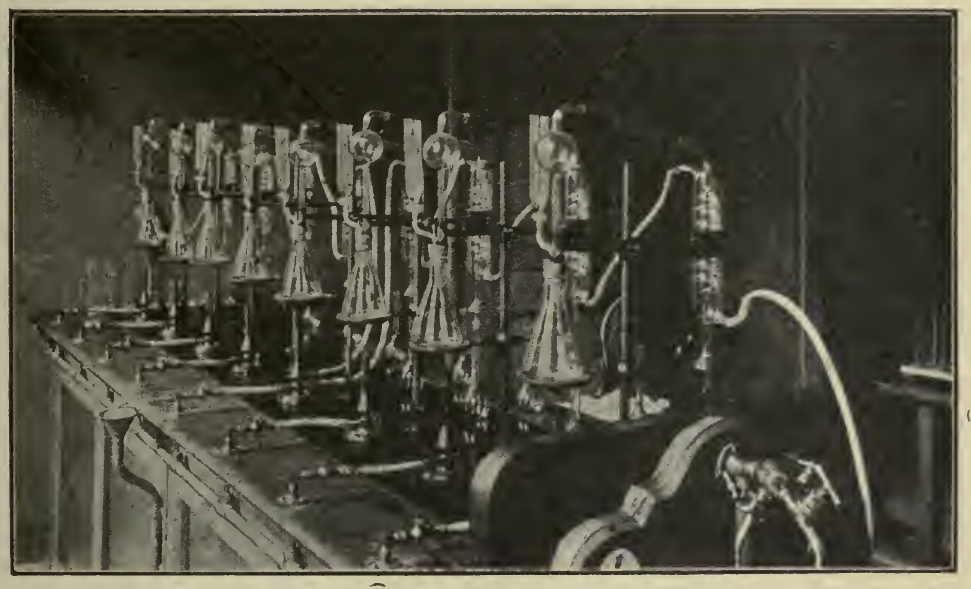

Fia. 61.-Distillation Apparatus for Reichert-Meissl Method.

being from 30 to 40 minutes. The distillate is then shaken, filtered through a dry filter, and titrated with $N / 10$ caustic soda solution in the same way as before.

Leffmann and Beam's modification is simpler and more convenient than the original method, and as it is quite as accurate it is to be preferred.

Whether the original or modified method be used, a blank experiment without the fat should always be made with the reagents which are to be used in the analysis. Generally a few tenths of a cubic centimetre of caustic soda are needed to neutralise the distillate, and this quantity must be deducted from the analysis. In Fig. 61 is shown the arrangement of 
condensers adopted in the laboratories where the Dutch butter supplies are tested. The Reichert-Meissl number may vary in the case of pure butter fat between 19 and 34, but only in rare instances does it fall below 24 (see below, under "Proof of Adulteration of Butter").

It is to be noticed that in the determination of the ReichertMeissl number the whole of the volatile, water-soluble, fatty acids in $5 \mathrm{~g}$. butter fat is not obtained, but only a portion (about 90 per cent.). The results, however, are constant for the same sample of butter, provided that the details of the method are carefully followed each time, and so the figures obtained from different samples of butter fat yield numbers which can be correctly compared one with another.

\section{The Koettstorfer Number.}

The Koettstorfer Number ${ }^{1}$ gives the number of milligrams of caustic potash which are required for the saponification of 1 g. fat.

The estimation is made by weighing out 1 or $2 \mathrm{~g}$. of clear filtered butter fat which has been well mixed into a 150 c.c. round-bottomed flask of Jena glass; 25 c.c. of an alcoholic solution of caustic potash, made by dissolving enough caustic potash in rectified spirit to make it about seminormal, are then added. The flask is closed with a cork through which a long glass tube, about $75 \mathrm{~cm}$. in length and $13 \mathrm{~mm}$. in diameter, is passed to act as an air condenser. It is preferable to choose a glass tube made from Bohemian potash glass for the purpose. The flask is then heated for 15 minutes on the water-bath, the contents being allowed to boil gently and shaken from time to time to accelerate the saponification. Whilst the contents of the flask are still warm they are titrated back with $N / 2$ hydrochloric acid, a few drops of alcoholic phenolphthalein being used as indicator. When the liquid becomes acid it changes suddenly to a yellow colour. By deducting the amount of acid required for this last titration from that which was needed to neutralise 25 c.c. of the original caustic potash, the amount of caustic needed for the saponification is obtained. This amount is then calculated

1 Zeitschrift für analyt. Chemie, 1879, Vol. XVIII, p. 199. 
in milligrams, per 1 gram butter fat. A blank experiment must be made alongside each determination.

For butter fat the Koettstorfer number generally lies between 220.5 and 232.0 .

\section{The Hehner Number.}

By the Hehner Number ${ }^{1}$ is understood the amount of insoluble fatty acids which is obtained from 100 parts of fat.

Three to four g. of pure butter fat are weighed out into a porcelain dish of about $10 \mathrm{~cm}$. diameter, and saponified with 1-2 g. of caustic soda in 50 c.c. alcohol, the dish being warmed on the water-bath and the contents well stirred. The solution of soap is evaporated to a thick syrup, then dissolved in water, and the soap decomposed by adding dilute sulphuric or hydrochloric acid until the reaction is strongly acid. The liquid is then heated until the fatty acids have collected as a clear oil on the surface, and then poured on to a dried and weighed filter made from very thick paper. Before using the filter it should be half filled with warm water, for this prevents the filtrate from being turbid. The fatty acids remaining on the filter are washed with small quantities of boiling water, the liquid not being allowed to fill the filter more than two-thirds full. When the washwater measures two litres the washing is discontinued, and the fatty acids are allowed to solidify. Finally the filter and the fatty acids are placed in a weighed glass beaker and dried in the oven at $100^{\circ} \mathrm{C}$. The amount of fatty acids is then calculated for 100 parts of fat.

The Hehner number for pure butter fat varies between 85.4 per cent. and 91.30 ; the average may be taken as 87.5 per cent.

\section{The Iodine Number.}

As is well known, certain unsaturated fatty acids (oleic, linoleic, \&c.) form addition compounds with iodine, $1 \mathrm{~mol}$. oleic acid (which is a component of butter fat), for example, taking up two atoms of iodine.

The iodine number of a fat is therefore understood to be the amount of iodine, expressed as percentage of fat, which the

1 Zeitschrift für analyt. Chemie, 1877, Vol. XVI, p. 145. 


\section{I54 MILK AND DAIRY PRODUCTS PART}

pure fat will absorb. Thus a measure of the amount of oleic acid (in the case of butter) present in the fat can be obtained.

\section{(a) Hübl's ${ }^{1}$ Method for Determining the Iodine Number.}

The required solutions are:

(1) Iodine-Mercuric Chloride.-Twenty-five g. of iodine in 500 c.c. of 95 per cent. alcohol (free from fusel oil); also $30 \mathrm{~g}$. of mercuric chloride in 500 c.c. of the same alcohol. The two solutions are kept separate, and at least forty-eight hours before being used equal quantities are mixed together. The mixture may be kept for some time, however, if 5 per cent. of concentrated hydrochloric acid (sp. gr. 1.19) is added to it. $^{2}$

(2) Sodium Thiosulphate.-This solution contains about $25 \mathrm{~g}$. per litre ; the exact strength can be determined by Volhard's method. $3 \cdot 874 \mathrm{~g}$. of pure potassium bichromate, which has been recrystallised several times, is dissolved in one litre of distilled water. 15 c.c. of a 10 per cent. potassium iodide solution are then brought into a bottle with ground-glass stopper along with 5 c.c. of concentrated hydrochloric acid and 100 c.c. of water. Then 20 c.c. of the bichromate solution are added under vigorous shaking. Each cubic centimetre of bichromate frees $0.01 \mathrm{~g}$. of iodine. The contents of the flask are then titrated with thiosulphate from a burette until the dark brown colour becomes faint yellow, at which point a few drops of starch paste are added, and the titration continued until the blue colour disappears.

As the thiosulphate rapidly changes, it must be standardised against the above-mentioned bichromate solution, which is very durable.

The actual determination of the iodine number is carried out as follows :

0.8-1 g. of clear filtered butter fat is weighed out into a specially designed flask with ground-glass stopper. $^{3}$ This flask, although it holds 270 c.c., only weighs from $40-50 \mathrm{~g}$. The fat is dissolved in 15 c.c. of chloroform, and 50 c.c. of the mixed iodine-mercury solution added from a burette. If the liquid, after stirring, is not quite clear, a little more chloroform must be added; and if, on the other hand, the colour becomes fainter after a short time, more iodine-mercury solution must be added. Enough iodine must be added to impart a dark brown colour to the liquid even after one and a half to two hours. Generally the reaction is complete at the end of several hours, but it is best to allow twenty-four hours to elapse, during which time the liquid is kept in a dark place at a temperature of $15-18^{\circ} \mathrm{C}$. At the end of this time 30 c.c. of 10 per cent. potassium iodide solution are added, the mixture

1 Dingler's Polyt. Journal, Vol. CCLIII, p. 281.

2 Waller, Chem. Zeitung., 1895, p. 1,831.

3 To be obtained from Joh. Greiner, Munich. 
shaken, and 100 c.c. water added. If a red precipitate of mercuric iodide should separate, then the amount of potassium iodide is insufficient and must be increased. Sodium thiosulphate is then run in from a burette until the colour of the solution, and also of the layer of chloroform, is only faintly yellow, at which point the starch paste is added. The iodine solution must be standardised afresh each time by titration against sodium thiosulphate, and the latter against potassium bichromate.

With each determination a blank must be made, the reagents being used exactly in the manner described above, but without the fat, of course. The result of the blank must be deducted when calculating the iodine number.

Most careful and uniform manipulation is required in carrying out the method described above, for the smallest error in the reading means a big difference in the final result. For this reason the same burettes, pipettes, \&c., must always be used.

Generally the iodine number for pure butter fat falls between 25.7 and 49 ; in the case of individual cows, in some special instances it may vary between 25 and 53 .

(b) Wijs' ${ }^{1}$ Method for Determining the Iodine Number.

Wijs found that in Hübl's solution it was the iodic acid which was the active component, and he sought to use this acid as the source of iodine. When, however, the solution came to be kept, and also during the reaction, difficulties arose owing to the decomposition of the acid. Wijs therefore chose a solution of iodine chloride in 99 per cent. acetic acid, and this can satisfactorily replace Huibl's solution. It is prepared by dissolving $13 \mathrm{~g}$. of iodine in 1 litre of acetic acid (Acidum aceticum glaciale 99 per cent. Merck) and the strength of the solution determined. Thereupon chlorine gas, which has been freed from hydrochloric acid by washing, is led slowly into the solution until the contents have doubled. This solution is a very constant one, and when it is used the time required for the experiment is only short, three to four or at most ten minutes. The acetic acid used in preparing the solution must be absolutely pure.

1 Ber. der deutsch. chem. Gesellschaft, 1898, p. 750. 
Another way of preparing the solution is to dissolve $9 \mathrm{~g}$. of commercial iodine trichloride in 1 litre of glacial acetic acid, and then titrate 5 c.c. of it with $N / 10$ sodium thiosulphate, first adding a few cubic centimetres of 10 per cent. potassium iodide solution and a little water. Afterwards sufficient finelypowdered iodine is dissolved in the solution of iodine trichloride to make the contents one and a half times as much as before. Instead of chloroform, Wijs uses carbon tetrachloride, which ought to be first shaken with a solution of potassium bichromate and concentrated sulphuric acid, the colour of which should not disappear even on standing for some time in contact with the tetrachloride. Wijs prefers carbon tetrachloride to chloroform because he has found that chloroform always contains some alcohol. The procedure is the same as in Huibl's method, save that only 10 c.c. of 10 per cent. potassium iodide solution are required, and half an hour is sufficiently long for the reaction to take place.

Wijs' method for determining the iodine number has come more and more into use during the last few years, for it has the advantage over Huibl's method in being simpler, quicker, and more constant in its results.

\section{Determination of the Amount of Free Acid.}

As it is interesting in many respects to know what quantity of free acid is present in butter, the following analytical method, prescribed by the German Imperial Health Authorities, is most generally used:

About $6 \mathrm{~g}$. of melted and filtered butter fat are weighed out and dissolved in an acid-free mixture composed of $15-20$ c.c. of alcohol and the same quantity of ether. The solution is then titrated with $N / 10$ alkali, and a 1 per cent. solution of alcoholic phenolphthalein used as an indicator. Every cubic centimetre of normal alkali which is required to saturate this free acid in $100 \mathrm{~g}$. butter fat is known as the degree of acidity. Some analysts calculate the total amount of free acid into oleic acid, and state what quantity of oleic acid, expressed in percentages of the weight of the butter fat, is equivalent to the free acid. One c.c. $N / 10$ alkali, as used in the above method, is equivalent to 0.0282 per cent, oleic acid $\left(\mathrm{C}_{18} \mathrm{H}_{34} \mathrm{O}_{2}=282\right)$. 


\section{Detection of the Adulteration in Butter.}

\section{Admixture of Foreign Fats.}

It is only to be expected that such a valuable and costly food as butter should be particularly liable to adulteration. The most usual way of adulterating butter, and at the same time often the most difficult to detect, consists in mixing with the butter a portion of cheaper and less valuable fat, either animal or vegetable. The main difference between butter fat and other fats lies in the fact that the former, whilst composed of glycerides of non-volatile fatty acids, viz., palmitin, stearin, and olein, as are other fats, contains also a number of glycerides of volatile, partly soluble, fatty acids of low molecular weight, viz., butyrin, capronin, caprylin, caprin. A full list of the fatty acids normally found in butter fat is the following:

$\begin{array}{lcccccccc}\text { Butyric acid } & \ldots & \ldots & \ldots & \ldots & & \mathrm{C}_{4} \mathrm{H}_{8} \mathrm{O}_{2} & \text { (volatile) } \\ \text { Caproic } & , & \ldots & \ldots & \ldots & \ldots & & \mathrm{C}_{6} \mathrm{H}_{12} \mathrm{O}_{2} & , \\ \text { Caprylic } & , & \ldots & \ldots & \ldots & \ldots & & \mathrm{C}_{8} \mathrm{H}_{16} \mathrm{O}_{3} & , \\ \text { Capric } & , & \ldots & \ldots & \ldots & \ldots & & \mathrm{C}_{10} \mathrm{H}_{20} \mathrm{O}_{2} & , \\ \text { Lauric } & , & \ldots & \ldots & \ldots & \ldots & & \mathrm{C}_{12} \mathrm{H}_{24} \mathrm{O}_{2} & \text { (non-volatile) } \\ \text { Myristic } & , & \ldots & \ldots & \ldots & \ldots & \mathrm{C}_{14} \mathrm{H}_{28} \mathrm{O}_{2} & , \\ \text { Palmitic } & , & \ldots & . & \ldots & \ldots & \mathrm{C}_{16} \mathrm{H}_{32} \mathrm{O}_{2} & , \\ \text { Stearic } & , & \ldots & \ldots & \ldots & \ldots & \mathrm{C}_{18} \mathrm{H}_{36} \mathrm{O}_{2} & , \\ \text { Arachidic } & , & \ldots & \ldots & \ldots & \ldots & \mathrm{C}_{20} \mathrm{H}_{40} \mathrm{O}_{2} & , ", \\ \text { Oleic } & , & \ldots & \ldots & \ldots & \ldots & & \mathrm{C}_{18} \mathrm{H}_{34} \mathrm{O}_{2} & ,\end{array}$

Lauric, myristic, and arachidic acids are only present in very small quantities. ${ }^{1}$ The glycerides of the first four of the above acids, which are volatile and characteristic of butter fat, usually make 7-9 per cent. of the total weight. The presence of these four glycerides in butter fat, particularly the butyrin, is, moreover, the only peculiarity which distinguishes it, as far as composition goes, from other fats. Therefore the admixture of foreign fat with the butter causes the non-volatile fatty acids to increase, whilst the quantity of volatile fatty acid diminishes. $\mathrm{As}$, however, the proportion of volatile fatty acids in butter is by no means constant, it is often extremely difficult, judging by the amount of volatile fatty acid, to decide whether or not falsification has taken place, particularly when only small quantities of foreign fat have been added. By far the majority of the methods which can be used to detect admixture of

1 According to Siegfeld (Zeitschr. für Untersuch. d. Nahr-und Gentssmittel, 1907, Vol. I, p. 513), butter fat contains less stearic and more myristic acid than was previously supposed. 


\section{$15^{8}$ MILK AND DAIRY PRODUCTS PART}

foreign fats with butter are nevertheless based upon the presence of these volatile fatty acids. Naturally, either the quantity of non-volatile fatty acids can be determined or the quantity of the volatile water-soluble acids. The former of these determinations is due to Hehner and Angell, ${ }^{1}$ and it was the first method used to distinguish butter fat from other fats. The second, the determination of the volatile acids, is the well-known Reichert-Meissl method. Koettstorfer's method for determining the number of acid molecules per unit weight of fat is based upon the same relation. All these methods have been fully described previously.

Only very bad cases of adulteration can be detected simply by one or the other of these methods, and generally several chemical and physical determinations must be used if an admixture with foreign fats is to be proved beyond doubt.

\section{(a) Admixture with Margarine.}

Margarine is an artificially prepared substitute for butter, the raw material from which it is made being beef suet. Shortly before the outbreak of the Franco-German war Napoleon III instructed the French chemist, Mège-Mouriès, to try to prepare a cheap substitute for butter, principally for army use. The method finally adopted by Mège-Mouriès remains unchanged in its chief features even to-day. Fresh suet is melted and separated from skin and connective tissue. The purified melted suet is known commercially as "premier jus," and if allowed to cool slowly after melting it separates into a granular mass with a high melting point, and an oily, low-melting portion. By hydraulic pressure at a temperature at which the oil does not solidify, the oily mass-the oleo-margarine-can be separated from the highmelting portion. The oleo-margarine is then churned with milk or cream to an emulsion, and afterwards rapidly cooled. By the addition of smaller or larger quantities of vegetable oil (generally earth-nut or cotton-seed), the consistency of the oleo-margarine can be regulated so that it becomes quite the same as that of butter.

Margarine, in its appearance, microscopic structure, and taste, resembles natural butter to such a high degree that it is almost

${ }^{1}$ Hehner and Angell, Butter, its Analysis and Adulterations, London, 1877. 
impossible to distinguish one from the other without a chemical or physical examination. The chemical difference consists naturally in the absence of volatile fatty acids, except the unimportant quantity which comes from the churning with milk or cream.

\section{Determination of the Refractive Index of the Fat.}

The figures which Wollny gives as limits between which the refractive index of natural butter varies are, as has previously been mentioned, $49 \cdot 5-54: 0$ divisions of the butter refractometer at $25^{\circ} \mathrm{C}$, and $40.5-44.4$ at $40^{\circ} \mathrm{C}$. Later investigations have, however, shown that these limits are too narrow, for the refractive index can sometimes rise to 47 divisions at $40^{\circ} \mathrm{C}$. Such exceptionally high refractive indices are usually found in connection with very low Reichert-Meissl numbers. The limits in the case of margarine fat are $58.6-66.4$ at $25^{\circ} \mathrm{C}$, and $48-52$ at $40^{\circ} \mathrm{C}$. An addition of margarine to butter may therefore be detected by a raising of the normal refractometer figure, provided that the adulteration is not so small that the refractive index of the mixture falls within the boundary of that for pure butter fat. If the number shown on the refractometer exceeds the maximum for butter, then the sample must be regarded as very suspicious. An examination with the refractometer cannot, however, be taken as a sufficient proof of falsification, but rather as an indication that a chemical examination ought to be made.

In the following are shown the maximum number of scale divisions-between $45^{\circ}$ and $25^{\circ} \mathrm{C}$. - that are permissible for pure butter.

Highest Allowable Refractometer Readings for Pure Butter at Different Temperatures.

\begin{tabular}{c|c||c|c|c|c}
\hline \multirow{2}{*}{ Temp. } & $\begin{array}{c}\text { Scale } \\
\text { divisions. }\end{array}$ & Temp. & $\begin{array}{c}\text { Scale } \\
\text { divisions. }\end{array}$ & Temp. & $\begin{array}{c}\text { Scale } \\
\text { divisions. }\end{array}$ \\
\cline { 2 - 3 } \cline { 5 - 6 } & & & & & \\
$45^{\circ}$ & $41 \cdot 5$ & $38^{\circ}$ & $45 \cdot 3$ & $31^{\circ}$ & $49 \cdot 2$ \\
44 & $42 \cdot 0$ & 37 & $45 \cdot 9$ & 30 & $49 \cdot 8$ \\
43 & $42 \cdot 6$ & 36 & $46 \cdot 4$ & 29 & $50 \cdot 3$ \\
42 & $43 \cdot 1$ & 35 & $47 \cdot 0$ & 28 & $50 \cdot 8$ \\
41 & $43 \cdot 7$ & 34 & $47 \cdot 5$ & 27 & $51 \cdot 4$ \\
39 & $44 \cdot 2$ & 33 & $48 \cdot 1$ & 26 & $51 \cdot 9$ \\
& $44 \cdot 8$ & 32 & $48 \cdot 6$ & 25 & $52 \cdot 5$ \\
\hline
\end{tabular}


To do away with the necessity of calculating by interpolation, Wollny has constructed a special thermometer on which the

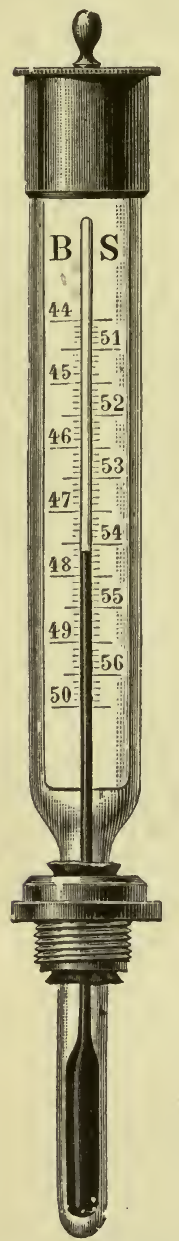

Fig. 62.-Wollny's Special Thermometer.

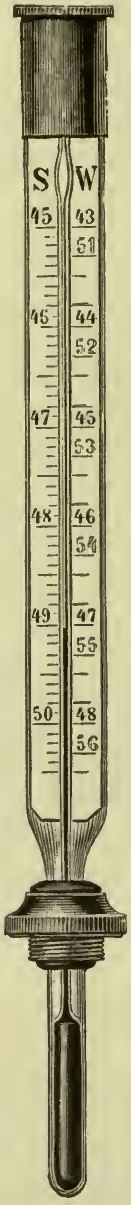

FIG. 63. - Baiers Special Thermometer. highestallowable number for a given temperature can be read.

In using this thermometer, it is sufficient to remember that if the refractometer scale gives a higher reading than the thermometer, the sample is suspicious, otherwise it may be regarded as pure. It must also not be forgotten that the limits set by Wollny have proved to be too low.

On this thermometer another scale $(S)$ for use with pigs' fat is also etched.

Another special thermometer has been designed to take into account the result of Baiers' ${ }^{1}$ investigations, for pure butter fat during summer feeding (June to October) has different limits from those of the winter months (November to May). The difference between the limits for summer and winter butter is as much as two divisions, summer butter giving the higher number. Wollny's scale, which is older, lies about intermediate between the summer and winter scale.

1 Zeitschrift. für Untersuch. der Nahr. - und Genussmittel, 1902, p. 1,145. 
In Baiers' special thermometer (Fig. 63), the left-hand side of the scale gives the figures in black for summer butter $(S)$, and the right-hand side for winter butter $(W)$. The scale for pigs' fat is on the right and in red figures.

It must not be forgotten that the critical line for natural butter is colourless on the refractometer scale, whilst that for margarine is blue, owing to the greater dispersive power of natural butter. Fat, which has a less dispersive power than butter, shows a red critical line on the refractometer.

\section{The Crismer Number.}

Whilst the critical temperature of solution of butter-fat in alcohol varies on an average between $53^{\circ}$ and $57^{\circ}$, as has already been seen (p. 145), the temperature in the case of margarine fat is $77^{\circ}-78^{\circ}$. An addition of margarine to natural butter may therefore be recognised by the raising of the Crismer number. There are, however, cases in which natural butter possesses an abnormally high Crismer number, so that great difficulty often arises in deciding whether adulteration with margarine has taken place, especially if the quantity added is only small.

\section{The Reichert-Meissl Number.}

Pure butter-fat has on an average a Reichert-Meissl number of 24-34, whilst that of the fat used in the manufacture of margarine is only $0 \cdot 1-0 \cdot 9$. In the process of manufacture these last figures may be raised to between 1 and 2 . The difference then between the Reichert-Meissl number of butter and that of margarine is considerable, and so it might be thought that this method would serve to detect quite small adulterations with margarine. It is, however, not to be forgotten that the volatile fatty acids present in butter may vary considerably owing to a number of causes, one of the chief of which is the individuality of the cow. Butter made from the milk of a single cow sometimes gives a very low Reichert-Meissl number, even as low as 13-14.

Further, the percentage of volatile fatty acids varies with the period of lactation, so that the Reichert-Meissl number is at its highest a few days after calving, and shortly afterwards 
begins to decline with regularity until the animal is almost dry. During this latter period, abnormally low figures are often obtained for the Reichert-Meissl number.

A ration rich in oil has often been proved to cause a fall in the Reichert-Meissl number, whereas one containing sugar raises it. ${ }^{1}$ When the cows begin to go out to grass the percentage of volatile fatty acids almost always falls, and remains lower during that time than during winter feeding. There are whole districts, for example, in Holland where the butter, particularly during the autumn months, shows a very low Reichert-Meissl number.

From what has been stated above, it is easy to see that to prove adulteration of butter with margarine may be very difficult, even though the Reichert-Meissl number may be unusually low. Where, however, the butter shows a ReichertMeissl number of less than 24, it must be regarded with suspicion, and further tests must be made to prove its genuineness.

\section{The Koettstorfer Number.}

Koettstorfer started from the fact that butter-fat, which contains a larger proportion of fatty acids of low molecular weight than other fats, must therefore contain more acid groups than do the latter. He therefore adopted as measure of this acid radicle the number of milligrams of caustic potash which are required for the saponification of $1 \mathrm{~g}$. of the fat under analysis. The Koettstorfer number fluctuates between 220.5 and 232, whilst margarine fat shows only 192-200. When a fat becomes rancid, the Koettstorfer number generally rises. When employed in conjunction with one another the Reichert-Meissl and the Koettstorfer numbers give very important indications as to whether or not a butter has been adulterated with margarine.

\section{The Phytosterine Acetate Test.}

On saponifying animal or vegetable fats there always remains some non-saponifiable material which in the case of the animal

1 Biedermann's Zentralblatt für Agrikulturchemie, 1905, p. 406. 
fat consists of cholesterine, whilst phytosterine is obtained from a vegetable fat. Salkowksi ${ }^{1}$ first proposed to make use of this fact as a means of detecting the admixture of vegetable fats with animal fats. It has been proved, chiefly through the researches of $\mathrm{A}$. Bömer, that phytosterine is always a constituent of plant fats, whilst cholesterine, on the other hand, is always found in animal fats. If, therefore, the presence of phytosterine can be proved in an animal fat-butter, for example-it is certain that adulteration with a vegetable fat has taken place.

As in the manufacture of margarine vegetable oils are always used; they being indispensable for obtaining the right consistency, it is easy to see that an addition of margarine to butter can be detected by the presence of phytosterine.

To distinguish phytosterine from cholesterine A. Bömer ${ }^{2}$ has worked out a method the main features of which are as follows The non-saponifiable portion from a certain quantity of fat is separated as completely as possible, and then allowed to slowly recrystallise from alcohol. In the original method the crystals obtained in this way were examined under the microscope, and the characteristic crystalline formation of the phytosterine or the cholesterine could be detected if either were present. When a mixture was being dealt with either the phytosterine crystallised out, or the crystals had a new and characteristic shape (Fig. 64).

This method, however, requires a certain proficiency in crystallographic measurements, and in addition it is difficult, where there is much cholesterine and only a little phytosterine, to detect the latter. Bömer, therefore, uses the acetate as a means of distinguishing the two substances, for the melting point of cholesterine acetate does not rise above $1154^{\circ}$, whilst that of the phytosterine acetate lies between 125 and $127^{\circ} .^{3}$ Even a small admixture of phytosterine acetate is recognised by the raising of the melting point. When making the test, $50 \mathrm{~g}$. of butter are saponified with 100 c.c. of alcoholic caustic potash ( $200 \mathrm{~g}$. of caustic potash, and 1 litre of $70 \mathrm{vol}$. per cent. alcohol), and then poured into a separating funnel with 200 c.c. of water.

1 Zeitschrift für analytische Chemie, Vol. XXVI, p. 557.

2 Zeitschrift für Untersuchung der Nahr. - und Genussmittel, 1898, 1901, 1902.

3 Siegfeld gives the melting point of phytosterine acetate as still higher. Zeitschrift für Untersuch. der Nahr.- und Genussmittel, 1904, No. 10, p. $57 \%$. 


\section{I64 MILK AND DAIRY PRODUCTS PART}

This liquid is shaken out once with 500 c.c. and two or three times with 200-250 c.c. of ether. After distilling off the ether
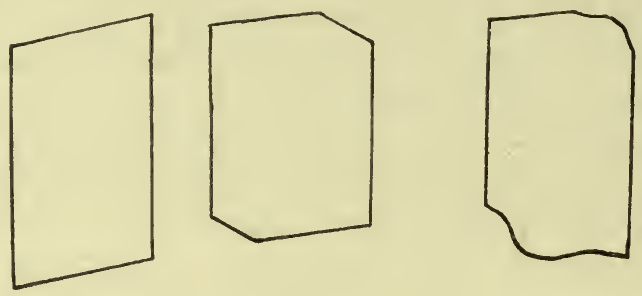

a
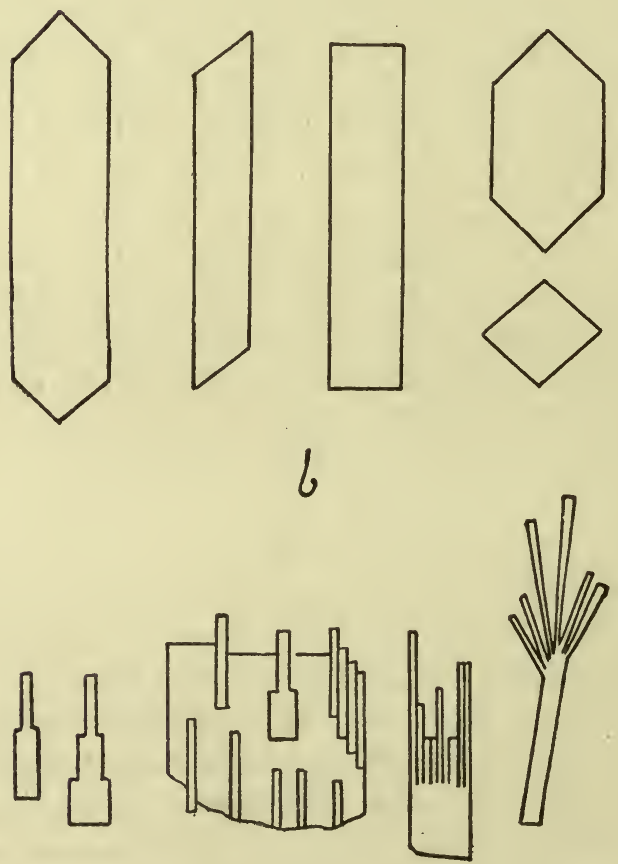

C

FIG. 64. - (a) Cholesterine Crystals; (b) Phytosterine Crystals; (c) Mixed Crystals of Cholesterine and Phytosterine.

the small quantity of alcohol that remains is got rid of by blowing a current of air into the flask. 
To make certain that no unsaponified fat remains, the residue in the flask is again saponified with 10 c.c. of caustic potash of the same strength as above, 20 c.c. of water being added, and the liquid shaken out with 80-100 c.c. of ether just as before. The ethereal solution is then washed with 5-10 c.c. of water, filtered, and the ether distilled off.

After the greater part of the ether has been got rid of in this way, the remaining ethereal solution is poured into a small glass crystallising dish, the ether completely evaporated off, and the residue dissolved in the least possible quantity of absolute alcohol, and allowed to crystallise out. The crystals which first separate are then subjected to a crystallographic examination. ${ }^{1}$ The remaining alcohol is then completely evaporated off on the water-bath, the dish placed on wire gauze, and after covering with a watch-glass the contents heated with 2-3 c.c. of acetic anhydride for about a quarter of a minute. After removing the watch-glass the excess of acetic anhydride can be got rid of on the water-bath.

The residue in the dish is then warmed with sufficient alcohol to dissolve the ester, and to avoid an immediate crystallisation on cooling a little more alcohol is added and the clear solution left to crystallise out. Until the temperature of the solution has sunk to that of the room the dish must be kept covered with a watch-glass. After half or two-thirds of the liquid has evaporated, and the greater part of the ester has crystallised out, the remainder is passed through a small filter, and the solid that remains in the dish is transferred, with the help of a spatula and a little 95 per cent. alcohol, to the filter. The contents of the filter are returned to the crystallising dish, dissolved in from 5 to 10 c.c. of absolute alcohol, according to the quantity, and again allowed to crystallise. After the greater part of the ester has crystallised out, it is filtered off and subjected to further recrystallisation in the same manner as long as sufficient material remains. After the third crystallisation the melting point of the ester is determined, and again after each subsequent recrystallisation, of which generally five to seven are necessary. If, after recrystallising the ester in the above way, the crystals last obtained do not melt completely at

1 Zeitschrift für Untersuchung der Nahrungs- und Genussmittel, 1898, Vol. I, pp. 42 and 44 ; Ibid, Vol. I, pp. 48 and 546. 
a lower temperature than $116^{\circ}$ (corrected m.p.) there is a possibility that vegetable fat has been added. When the ester melts only at $117^{\circ}$ (corrected m.p.) or above that temperature, then the addition of vegetable fat may be said to be proved.

When using this method the melting point must always be corrected, which may be done with sufficient accuracy from the following formula :

$$
M=T+n(T-t), 0 \cdot 000154 .
$$

$M=$ the corrected melting point.

$T=$ the observed melting point.

$n=$ the length of the column of mercury, expressed in degrees of temperature, above the level of the liquid.

$t=$ the temperature of the air surrounding the column of mercury above the level of the liquid.

Siegfeld ${ }^{1}$. has shown that by means of this method an adulteration with 10 per cent. margarine or cocoa butter can be proved with certainty if $50 \mathrm{~g}$. of the butter are used.

The phytosterine acetate test is certainly somewhat tedious to carry out, but when properly done it gives absolutely incontrovertible results, for it is based on the fact that pure butterfat only contains cholesterine and never phytosterine. Also the melting point of phytosterine acetate is considerably higher than cholesterine acetate, so that a small admixture of phytosterine must make itself apparent by a rise in the melting point.

In making the test $100 \mathrm{~g}$. of fat can of course be used, and this is more convenient, but with practice $50 \mathrm{~g}$. will be found to be quite sufficient.

\section{The Latent Colouring of Margarine.}

On account of the difficulty in detecting the addition of margarine to butter, either by chemical or physical tests, it has been decreed in certain countries, e.g., Germany, Austria, Belgium, Denmark, Sweden, and France, that in the manufacture of margarine some easily recognisable material must be added. By means of a simple chemical test it is then easy to prove the presence of margarine in butter.

1 Zeitschrift für Untersuchung der Nahr.- und Genussmittel, 1904, No. 10, p. 577 . 
The simplest way of course would be to mix some substance with the margarine, which, without diminishing its value, could easily be detected by a characteristic colour reaction (latent coloration). Several of such additives have been proposed, viz., sesamé oil, phenolphthalein, starch, but the only one that has been at all generally adopted is sesamé oil, which was first proposed by $\mathrm{H}$. Bremer. ${ }^{1}$ This substance can easily be detected by the red colour which it gives with furfurol and hydrochloric acid, a test due to Baudouin ${ }^{2}$ and modified by Villavecchia and Fabris. ${ }^{3}$ All the countries which insist upon the addition of easily distinguishable substances to margarine prescribe sesamé oil (Belgium starch as well).

Sesamé oil, which was first used for this purpose in 1897 in Germany, is particularly suitable, because it can take the place of other vegetable oils used in the manufacture of margarine. The prescribed amount of sesamé oil in margarine is 10 per cent., and the oil must show the following reactions: If to a mixture of 0.5 part by volume of sesamé oil and 99.5 parts by volume of cotton-seed oil or earth-nut oil there be added 100 parts of fuming hydrochloric acid (sp. gr. 1.19) and a few drops of a 2 per cent. alcoholic solution of furfurol, the hydrochloric acid below the layer of oil must show a distinct red colour. The furfurol used in the test must be colourless.

The details of this so-called Baudouin's test are as follows: 5 c.c. of melted butter fat are violently shaken with $0 \cdot 1$ c.c. of an alcoholic furfurol solution (1 part of colourless furfurol and 100 parts of absolute alcohol) and 10 c.c. of hydrochloric acid (sp. gr. 1.19). When the hydrochloric acid which separates out below has a distinct red colour which does not quickly disappear, the presence of sesamé oil has been proved. Should there be any colouring matter in the butter which would turn red in the presence of hydrochloric acid, it must first be shaken with hydrochloric acid (sp. gr. 1.125) to free it from the colouring matter. In certain cases it happens that when shaken with hydrochloric acid Baudouin's test does not give any red coloration, even though sesamé oil be present. In doubtful cases Fendler ${ }^{4}$ proposes that a method employed by

1 Milch-Zeitung, 1897, p. 210.

2 Zeitschrift für das chemische Grossgewerbe, 1878, p. 711.

3 Zeitschrift fïr angeurendte Chemie, 1893, p. 505.

+ Chem. Revue, Vol. XII. 
Soltsien ${ }^{1}$ should be used to test for the sesamé oil. In this method the fat is shaken with twice its volume of benzine and half its volume of stannous chloride $\left(\mathrm{SnCl}_{2}\right)$ solution until the liquids are thoroughly mixed, then placed in a water-bath at a temperature of $40^{\circ}$, which is raised to $80^{\circ} \mathrm{C}$. when the sediment has settled. During this time the level of the water-bath must not be higher than the level of the benzine layer, and every care taken to prevent the benzine from boiling. In the presence of sesamé oil the stannous chloride turns red.

\section{(b) Admixture with Cocoa-nut Oil.}

A special fat, known as cocoa-nut oil or cocoa-nut butter, can be extracted from the kernel of the fruit of the cocoa-nut palm (Cocos nucifera). In the last few years the fat has come largely into use as a food. When fresh, it is colourless and resembles lard, but the taste and smell are peculiar and characteristic. By means of a simple process, however, the taste and smell can be completely removed, so that this cocoa-nut fat may be used instead of butter in the preparation of food. Owing to its cheaper price, cocoa-nut butter is now largely used in cooking, \&c., in France, England, and Germany. On account of the properties just mentioned, cocoa-nut fat has been used more and more for the adulteration of butter. Food analysts have, therefore, been obliged to devise methods for the detection of this fat in butter. These tests are based upon the characteristic composition and peculiar properties of cocoa-nut butter.

Two of the most commonly employed and most reliable tests used in the analysis of butter-the determination of the refractive index and of the volatile water-soluble fatty acids (the Reichert-Meissl number) — cannot satisfactorily be employed in this case. This is because the refractive index of cocoanut oil, $33.5-35.5$ divisions at $40^{\circ}$, lies too near that of butter itself to show any but a large adulteration. As regards the Reichert-Meissl number, the cocoa-nut oil contains an unusually high percentage of volatile acids ${ }^{2}$ compared with other fats, except butter. The Reichert-Meissl number of a cocoa-nut oil lies between 6 and 8 , and it is therefore clear that the

1 Zeitschrift für öffentliche Chemie, 1897, Vol. III, p. 495 ; also Pharm. Zeit., 1901, Vol. XLVI, p. 850

${ }_{2}^{2}$ Between $\mathrm{C}_{6}$ and $\mathrm{C}$ 
addition of a small quantity of this fat is very difficult to detect, particularly as the Reichert-Meissl number of natural butter has had to be lowered considerably in recent times. A good indication of butter adulterated with cocoa-nut oil is the relation between the iodine number and the Koettstorfer number, for then the former is very low and the latter very high. The iodine number of cocoa-nut fat lies between 8 and 9.5 , and the Koettstorfer number 253-271. Crismer's number for cocoa-nut fat is $31^{\circ}$. The special tests used for the detection of cocoanut oil in butter are the following:

\section{Polenske's New Butter Number.}

Polenske's method ${ }^{1}$ is based upon the well-known fact that the majority of the volatile acids in butter fat are soluble in water, whilst those in cocoa-nut oil are insoluble. Further, as has already been mentioned, the Reichert-Meissl number for cocoa-nut fat is from $6-8$, whilst the amount of $N / 10$ caustic soda which is required to neutralise the insoluble fatty acids which pass over in the same distillation is from $16-18$ c.c.

Polenske titrates the insoluble volatile fatty acids obtained in the Reichert-Meissl method, and calls the number of cubic centimetres of N/10 caustic soda which are used the "New Butter Number." It would be more suitable to call this figure the Polenske number, and this will be done whenever further mention is made of the method.

The Polenske number for pure butter fat stands in a certain relation to the Reichert-Meissl number as long as the latter does not exceed 27 , but above that figure the same ratio is not kept. For figures below 27, the Polenske number can be got from the Reichert-Meissl number by means of the formula:

$$
\frac{\text { R.-M.N. }-7}{10}=\text { Polenske number. }
$$

For a Reichert-Meissl number above 27, the figure 7 must be replaced by 5 .

Polenske has drawn up a table showing the relation between the Reichert-Meissl and Polenske numbers in the case of pure butter fat, which is as follows:

1 Arbeiten uus dem Kaiserl. Gesundheitsamte, Vol. XX, p. 545; Zeitschrift für Untersuch. der Nahr. - und Genussmittel, 1904, Vol. I, p. 273. 


\begin{tabular}{c|c|c}
\hline $\begin{array}{c}\text { Reichert-Meissl } \\
\text { number. }\end{array}$ & Polenske number. & $\begin{array}{c}\text { Highest allowable } \\
\text { figure. }\end{array}$ \\
\cline { 2 - 2 } $20-21$ & $1 \cdot 3-1 \cdot 4$ & $1 \cdot 9$ \\
$21-22$ & $1 \cdot 4-1 \cdot 5$ & $2 \cdot 0$ \\
$22-23$ & $1 \cdot 5-1 \cdot 6$ & $2 \cdot 1$ \\
$23-24$ & $1 \cdot 6-1 \cdot 7$ & $2 \cdot 2$ \\
$24-25$ & $1 \cdot 7-1 \cdot 8$ & $2 \cdot 3$ \\
$25-26$ & $1 \cdot 8-1 \cdot 9$ & $2 \cdot 4$ \\
$26-27$ & $1 \cdot 9-2 \cdot 0$ & $2 \cdot 5$ \\
$27-28$ & $2 \cdot 0-2 \cdot 2$ & $2 \cdot 7$ \\
$28-29$ & $2 \cdot 2-2 \cdot 5$ & $3 \cdot 0$ \\
$29-30$ & $2 \cdot 5-3 \cdot 0$ & $3 \cdot 5$ \\
\hline
\end{tabular}

The addition of cocoa-nut oil to the extent of:

10 per cent. raises the Polenske number $0 \cdot 8-1 \cdot 2$ average 1.0 .

15

20

$$
\begin{aligned}
& \text { " } \quad 1.4-1.8 \quad " \quad 1.6 \text {. } \\
& \text { " } \quad 1.9-2 \cdot 2 \quad \text { " } 1.9 \text {. }
\end{aligned}
$$

If, then, the Polenske number is higher in relation to the Reichert-Meissl number than is given in the above table, there is an indication that cocoa-nut fat has been added, for this is the effect that it has.

The details of the method are exactly the same as those of Leffman and Beam's modification of the Reichert-Meissl method, but in order to obtain comparable figures very great care must be exercised in following Polenske's directions as to size of distillation flask and condenser, temperature of condenser water, time of distillation, \&c. In Fig. 65 is shown the arrangement and size of the distillation apparatus, and it will be noticed that the tube passing through the cork in the neck of the flask has an opening in the side.

Five g. of clear filtered butter fat are saponified as usual with 20 g. of glycerine and 2 c.c. of caustic soda $(1: 1)$ in a 300 c.c. Jena glass flask over a naked flame, and the soap dissolved in 90 c.c. of water that has previously been boiled. The solution must be quite clear and colourless, or at most have a yellow tinge ; so on this account all the fats that give a brown solution cannot be investigated. The soap solution, having been warmed to about $50^{\circ}$, is then decomposed with 50 c.c. of dilute sulphuric acid (25 c.c. per litre), a little powdered pumice stone added (not pieces of pumice stone), and the flask immediately connected to 
the condenser and the distillation proceeded with. The flame must be so regulated that the 110 c.c. of distillate pass over in from 19-21 minutes, and the temperature of the condenser must be so adjusted that the distillate is neither too warm nor too cold, but enters the flask at from 20 $23^{\circ}$. Wiedemann ${ }^{1}$ has proposed the following arrangement for easily obtaining a constant temperature: Rubber tubing is put through the inlet and outlet tubes of the Liebig condenser and pushed along to within a few centimetres of where the side tubes are fixed. Cold water entering from below is driven up the rubber tubing into the upper part of the condenser, but sinks owing to its greater density to the lower part of the condenser, where it then reaches the mouth of the rubber tubing going to the outlet, and so flows away. By

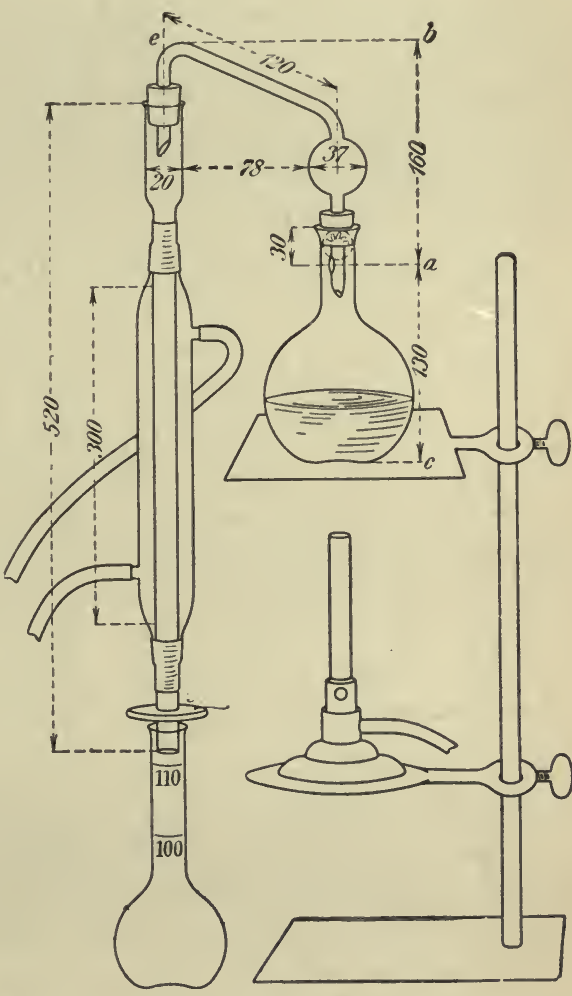

Fig. 65. - Polenske's Distillation Apparatus. this means the temperature of the condenser water can be kept almost constant at the desired temperature.

As soon as the distillate reaches 110 c.c. the flame is removed, and the receiving flask replaced by a 25 c.c. measuring cylinder. The flask containing the distillate is placed, without being shaken, for ten minutes in water of $15^{\circ} \mathrm{C}$, and sunk until the 110 c.c. mark is some $3 \mathrm{~cm}$. below the water level. At the end of the first five minutes the flask is moved to and fro but not so vigorously

1 Molkerei-Zeitung, Hildesheim, 1904, p. 681. 
as to cause the particles of solid acid floating on the surface to adhere to the neck. After ten minutes' cooling the physical condition of the fatty acids is observed, and it is noted whether a solid or semi-solid opaque shapeless mass is present, or whether there are clear drops of oil (cocoa-nut fat.) ${ }^{1}$

After closing the neck of the flask with a glass stopper, the distillate is mixed by inverting the flask four or five times, after which the contents is filtered. The Reichert-Meissl number of the filtrate is then determined in the usual manner. The filter must have a diameter of $8 \mathrm{~cm}$., and must fit close to the sides of the funnel.

After the distillate has completely filtered through, the filter is washed three times with 15 c.c. water, the filter being filled each time to the top. This wash water must first be used to wash out thrice the condenser tube, the measuring cylinder and the receiver. When all of the wash water, the last 10 c.c. of which should be neutralised by one drop of $N / 10$ baryta water, has filtered through, exactly the same operations are repeated with neutral 90 per cent. alcohol, 15 c.c. being used each time.

The fatty acids in the united alcoholic filtrates are then titrated with $N / 10$ baryta water, three drops of phenol phthalein being used as indicator, and the number of cubic centimetres which are required gives the Polenske number.

The standard numbers given by Polenske must not be regarded as absolute. Hesse ${ }^{2}$ has shown that considerable variations can occur in the relation between the ReichertMeissl and Polenske numbers, although the latter is seldom below and often above that given by Polenske himself.

Liihrig ${ }^{3}$ and Siegfeld ${ }^{4}$ have found that feeding with cocoa-nut cake and still more so with beet leaves causes the Polenske number to rise. It cannot, however, be denied that the Polenske number gives very valuable information, even though it does not always suffice to prove whether or not a butter is adulterated with cocoa-nut fat. Müntz and Coudon ${ }^{5}$ published, at about the same time as Polenske did, a method of detecting

1 These drops of oil contain caprylic acid, which is characteristic of cocoanut fat.

2 Milchwirtschaftliches Zentrulblatt, Vol. I, No. 1, 1905.

3 Zeitschrift für Unter. d. Nahr.- und Genussmittel, 1906, Vol. 11, p. 11.

4 Ibid., 1909, Vol. XVII, p. 177 ; Chemiker-Zeitung, 1907, No. 40.

${ }^{5}$ Bull. mensuel de l'Office de renseignements agricoles, Fevr. 1904, p. 147. 
the presence of cocoa-nut fat in butter, but as it does not differ in principle from Polenske's, it will not be described in detail here. It will suffice to mention that the details in apparatus and procedure must always have the closest attention, for the constants with which the results of the analysis are to be compared differ according to the method of performing the experiment. Naturally a great number of such methods can be worked out, but if the time of distillation of the volatile acids or of the whole analysis is varied, then a long series of tests with pure butter and with cocoa-nut fat have to be made, in order to determine the normal fluctuations in the amount of soluble and insoluble fatty acids. Further, the figures which are obtained from known mixtures of both fats must also be ascertained. Miintz and Coudon express the relation between the insoluble and the soluble fatty acids in the following way:

$$
\frac{\text { Insoluble acids }}{\text { Soluble acids }} \times 100 \text {. }
$$

In the case of pure butter the ratio is from 10-15, whilst with cocoa-nut fat it lies between 250 and 280 , a very considerable difference.

Latterly a number of other methods for the detection of cocoa-nut fat in butter have been proposed. None of them, however, have withstood the rigorous tests to which they have been subjected. Perhaps the cadmium method recommended by Paal and Amberger ${ }^{1}$ will prove to give reliable results, but it has not yet been sufficiently tested to allow of a definite verdict being pronounced.

\section{Qualitative Tests for Cocoa-nut fat in Butter.}

The phytosterine test (p. 162) gives absolutely reliable results in so far as it shows the addition of some vegetable fat, but it does not prove what particular fat has been added. The two following methods can be used to identify cocoa-nut fat, and where the adulteration has been considerable the results are very reliable. Both methods are based upon the characteristic shape which the cocoa-nut fat assumes when it crystallises out. Soltsien's ${ }^{2}$

\footnotetext{
1 Zeitschr. für Untersuch. d. Nahr.- und Genussmittel, 1909, Vol. XVII, pp. 1 and 23 .

${ }_{2}$ Augsburger Seifen-Zeitschrift, 1904, p. 898.
} 
test is made by allowing the fat which has been melted at $20^{\circ} \mathrm{C}$. to slowly crystallise. Characteristic spherical aggregates of crystals are formed, and these give a peculiar marbled appearance to the fat. When the sample only contains a little cocoa-nut fat, it is best to make the test with an alcoholic extract of the fat.

A. Mercier ${ }^{1}$ also recommends a test based upon the same lines. To 1 c.c. of melted fat are added 30 c.c. of $90 \%$ alcohol and digested for five minutes at $50^{\circ}-55^{\circ} \mathrm{C}$. Then the fat and alcohol are well mixed, allowed to stand for 15-20 minutes and 20 c.c. decanted into a test-tube. After cooling to $30^{\circ}-40^{\circ}$ and filtering, the fat is allowed to slowly crystallise out. The crystals are then filtered off and examined microscopically. Cocoa-nut fat is shown by little bunches of crystals resembling a powder puff, the individual crystals being long needles.

In some cases the adulteration of butter with cocoa-nut oil can be proved by an examination of the fat under the polarising microscope. Butter fat that has been allowed to cool very slowly then crystallises in spherical crystals which appear to have a dark cross on them. Cocoa-nut fat, on the other hand, crystallises, as has been mentioned, in long fine needles which are uniformly illuminated under the polarising microscope. In most cases the adulteration has been so skilfully done that the characteristic shape of the fat has been purposely destroyed by rapid cooling and subsequent agitation.

Adulteration of butter with other animal or vegetable fats than margarine or cocoa-nut oil also occurs, but at the present time there are hardly any satisfactory analytical methods for their detection, and where the falsification has been cleverly done it is almost impossible to devise a means of detecting it.

The whole of this branch of the subject contains problems which are very difficult of solution by the analyst. At first it was thought that by adopting a certain standard for butter it would be possible to regard as adulterated those samples that did not fall within the limits of this standard. As the analytical material increased, however, the necessity of modifying the standards arose, for often a natural butter quite free from foreign fat was shown to have a composition

1 Bull. du Service de Surveillance de la Fabr. et du Commerce des Denrées alimentaires, 1904, p. 873. 
which varied considerably from the figures given as standards for normal butter. Thus the lower limit for the ReichertMeissl number of natural butter had gradually to be reduced from 28 to 24, and finally to 19 . In the same way, the iodine number was put at 38 for the maximum, but it is now known that it sometimes rises to 50 . With regard to the refractive index, it has previously been mentioned (p. 159) that the figures given by Wollny are altogether too low, for he states that $44 \cdot 2$ divisions at $40^{\circ} \mathrm{C}$. is the highest number to be allowed for pure butter, whereas it has been found that 47 divisions may be got with pure butter.

It will thus be seen that sometimes the chemist has almost insuperable difficulties to overcome if he wishes to prove in some particular case whether or not butter has been adulterated. The best safeguards against adulteration are, and will continue to be, legal standards and a strict control in the preparation and sale of the so-called artificial butters.

Synopsis of the Most Important Analytical Data for Butter, Margarine and Cocoo-nut Fat.

\begin{tabular}{|c|c|c|c|c|c|}
\hline & & & Butter. & Margarine. & Cocoa-nut fat. \\
\hline $\begin{array}{l}\text { Specific gravit } \\
\text { Melting point, } \\
\text { Solidifying poi } \\
\text { Crismer numb } \\
\text { Refractive ind } \\
\text { Reichert-Meis } \\
\text { Polenske } \\
\text { Köttstorfer } \\
\text { Hehner } \\
\text { Hübl's Iodine }\end{array}$ & $\begin{array}{l}100^{\circ} \mathrm{C} . \\
\ldots . \\
{ }^{\circ} \mathrm{C} . \\
\mathrm{C} . \\
40^{\circ} \mathrm{C} . \\
\text { umber } \\
, \\
, \\
, \\
,\end{array}$ & $\begin{array}{l}\ldots \\
\ldots \\
\cdots \\
\ldots \\
\cdots \\
\cdots \\
\cdots \\
\cdots \\
\cdots \\
\cdots\end{array}$ & \begin{tabular}{|c|}
$0 \cdot 865-0 \cdot 968$ \\
$28-36$ \\
$19-23$ \\
$53-57(59)$ \\
$40 \cdot 5-45(47)$ \\
$(19) 24-34$ \\
$1 \cdot 3-5 \cdot 3$ \\
$220 \cdot 5-232$ \\
$85 \cdot 4-91 \cdot 3$ \\
$25 \cdot 7-49$
\end{tabular} & $\begin{array}{c}0 \cdot 859-0 \cdot 860 \\
32-35 \\
20-22 \\
77-78 \\
48-52 \\
0 \cdot 1-0 \cdot 9 \\
0 \cdot 35-0 \cdot 6 \\
190-200 \\
94-96 \\
48-64\end{array}$ & $\begin{array}{c}0 \cdot 869 \\
20-28 \\
15 \cdot 7-23 \\
31 \\
33 \cdot 5-35 \cdot 5 \\
6-8 \\
16 \cdot 8-17 \cdot 8 \\
253-271 \\
81 \\
7 \cdot 9-9 \cdot 5\end{array}$ \\
\hline
\end{tabular}

\section{Mixing of Water with Butter.}

Butter churned and prepared in the usual way contains 11-15 per cent. water, but the percentage can easily be raised by special methods, and may even reach 30 per cent. without the appearance arousing suspicion. The object, of course, of incorporating such large quantities of water is to increase the weight of the butter, so the practice must be regarded as a 
form of adulteration. The maximum quantity of water which is allowed to be present in butter offered for sale is fixed by law in many countries. Fortunately this form of falsification is easily detected by simply determining the percentage of water in the butter (p. 135).

\section{Addition of Preservatives.}

In many countries the addition of preservatives to butter is legally forbidden, the only exception being made in the case of common salt, which is everywhere allowed. Boracic acid and its salts are the most usual preservatives, but occasionally some of those mentioned as preservative materials for milk (p. 119) are met with. As regards analytical methods for the detection of preservatives in butter those described for milk are generally applicable. The fat must first be got rid of as completely as possible by melting the butter and carefully separating the water from it in a separating funnel. The water can then be tested in the usual way. Relatively smaller quantities are to be taken than is the case in the analysis of milk.

\section{Detection of Colouring Matter in Butter.}

The colouring of butter by the addition of some colouring matter is now so general and so largely practised that hardly anybody stays to consider whether or not such a course is morally right or not. Artificial colouring of butter has as its object the attainment of the bright yellow colour which is naturally present during the summer months. Only very small quantities of harmless colouring matters are used as a rule, so that there has not been any necessity for steps to be taken against the practice. The public has become accustomed to buying butter of the same colour all the year round, so that it is difficult to abolish the practice.

It therefore only becomes necessary to see that the colouring of the butter is not done with any poisonous or injurious substances.

Until the last few years butter was always coloured with vegetables dyes, and the best of that class is annatto, which is got from a South American tree, Bixa orellana. The dye is got by vigorously stirring up the ripe and open fruit capsules 
with water, straining off the liquid and the dye-stuff floating in it and boiling them together.

Annatto is a solution of the dye-stuff in sesamé oil or in fine olive oil. 'The dye is brownish-yellow, but when diluted has a beautiful bright yellow colour. Other substances which are used for colouring butter are safflowers, marigolds, saffron, and curcuma.

As the vegetable colouring substances are sensitive to light - that is, they are bleached when exposed to light-and as the Bixa orellana is becoming more and more rare, the practice of using artificial dye-stuffs such as aniline yellow-dimethylamidoazobenzol (butter yellow), Martius yellow-dinitro-, naphthol, Victoria yellow-potassium dinitrocresolate, \&c., has commenced in the last few years. The question whether such aniline colours should be allowed is still under discussion, although the colours which come into question are not poisonous. A certain test for aniline dyes in butter colouring is simply to shake the melted butter with hydrochloric acid, sp. gr. 1.125, and the presence of such dyes is shown by the acid becoming red.

A complete scheme for investigating butter colouring has been worked out by A. R. Leeds, ${ }^{1}$ who advises the following procedure: $100 \mathrm{~g}$. of the butter-natural or artificial-are dissolved in $300 \mathrm{~g}$. of pure petroleum ether (sp. gr. 0638) and then separated from the water and salts in solution by means of a separating funnel. The petroleum ether containing the fat is washed several times with water, 100 c.c. in all, and then allowed to stand for fifteen to twenty hours in ice water in summer, or in a cold place in winter, whereby a considerable quantity of stearin crystallises out. The clear solution is decanted from the stearin and shaken with 50 c.c. of $N / 10$ standard alkali, which withdraws the colouring matter from the petroleum ether. The aqueous solution containing the colouring matter is separated from the fat solution and carefully acidified with dilute hydrochloric acid until the liquid has a distinct acid reaction to litmus paper. Thereupon the colouring matter (along with some fatty acid) separates out, and it is then filtered through a tared filter and washed with cold water. It must not be forgotten that the petroleum ether

1 The Analyst, 1887 ; Chem. Zeitung Repert., 1887, p. 188. 
solution always has a bright yellow colour which comes from the fat.

In the following table the reactions characteristic of the different colouring substances are shown. An alcoholic solution of the colouring matter is always used, and only two to three drops of the solution and of the reagent are required.

Ammonia is coloured reddish-brown by curcuma, but after evaporation of the ammonia the original colour returns.

Synopsis of the Reactions which are characteristic of the various Butter Colourers (Leeds).

\begin{tabular}{|c|c|c|c|c|}
\hline Dye. & $\begin{array}{c}\text { Concentrated } \\
\mathrm{H}_{2} \mathrm{SO}_{4} \text {. }\end{array}$ & $\begin{array}{c}\text { Concentrated } \\
\mathrm{HNO}_{3} \text {. }\end{array}$ & $\begin{array}{l}\mathrm{H}_{2} \mathrm{SO}_{4}+ \\
\mathrm{HNO}_{3} .\end{array}$ & $\begin{array}{c}\text { Concentrated } \\
\text { HCl. }\end{array}$ \\
\hline Annatto & $\begin{array}{l}\text { Indigo blue } \\
\text { to violet. }\end{array}$ & $\begin{array}{l}\text { Blue, colour- } \\
\text { less on stand- } \\
\text { ing. }\end{array}$ & $\begin{array}{l}\text { Blue, colour- } \\
\text { less on stand- } \\
\text { ing. }\end{array}$ & $\begin{array}{l}\text { No change, } \\
\text { only slightly } \\
\text { dirty yellow } \\
\text { or brown. }\end{array}$ \\
\hline $\begin{array}{l}\text { Annatto and } \\
\text { decolorised } \\
\text { butter. }\end{array}$ & $\begin{array}{l}\text { Blue, passing } \\
\text { through green } \\
\text { to violet. }\end{array}$ & $\begin{array}{l}\text { Blue, passing } \\
\text { through green } \\
\text { to colourless. }\end{array}$ & Decolorised. & $\begin{array}{l}\text { No change, } \\
\text { only slightly } \\
\text { dirty yellow. }\end{array}$ \\
\hline Curcuma $\quad .$. & Pure violet. & Violet. & Violet. & $\begin{array}{l}\text { Violet, on } \\
\text { evaporation of } \\
\text { the HCl the } \\
\text { original colour } \\
\text { returns. }\end{array}$ \\
\hline $\begin{array}{l}\text { Curcuma and } \\
\text { decolorised } \\
\text { butter. }\end{array}$ & $\begin{array}{l}\text { Violet to } \\
\text { purple. }\end{array}$ & $\begin{array}{l}\quad \text { Violet to } \\
\text { reddish } \\
\text { purple. }\end{array}$ & $\begin{array}{l}\text { Violet to } \\
\text { reddish pur- } \\
\text { ple. }\end{array}$ & $\begin{array}{l}\text { Very beanti- } \\
\text { ful violet. }\end{array}$ \\
\hline Saffron .. ... & $\begin{array}{l}\text { Violet to co- } \\
\text { balt blue, be- } \\
\text { comes reddish } \\
\text { brown. }\end{array}$ & $\begin{array}{l}\text { Light blue, } \\
\text { becomes light } \\
\text { reddish brown. }\end{array}$ & $\begin{array}{l}\text { Light blue, } \\
\text { becomes light } \\
\text { reddish brown. }\end{array}$ & $\begin{array}{l}\text { Yellow, be- } \\
\text { comes dirty } \\
\text { yellow. }\end{array}$ \\
\hline $\begin{array}{l}\text { Saffron and } \\
\text { decolorised } \\
\text { butter. }\end{array}$ & $\begin{array}{l}\text { Dark blue, } \\
\text { rapidly be- } \\
\text { comes reddish } \\
\text { brown. }\end{array}$ & $\begin{array}{l}\text { Blue, passing } \\
\text { through green } \\
\text { to brown. }\end{array}$ & $\begin{array}{r}\text { Blue, rapidly } \\
\text { becomespurple. }\end{array}$ & $\begin{array}{l}\text { Yellow, be- } \\
\text { comes dirty } \\
\text { yellow. }\end{array}$ \\
\hline Carrots & Dark brown. & Decolorised. & $\begin{array}{l}\text { Gives } \mathrm{NO}_{2} \\
\mathrm{f} \text { umes and } \\
\text { smell of burnt } \\
\text { sugar. }\end{array}$ & $\begin{array}{l}\text { Not decol- } \\
\text { orised. }\end{array}$ \\
\hline $\begin{array}{l}\text { Carrots and } \\
\text { decolorised } \\
\text { butter. }\end{array}$ & \begin{tabular}{l}
\multicolumn{1}{c}{ Reddish } \\
brown to pur- \\
ple, similar to \\
curcuma.
\end{tabular} & $\begin{array}{l}\text { Yellow and } \\
\text { decolorised. }\end{array}$ & $\begin{array}{l}\text { Yellow and } \\
\text { decolorised. }\end{array}$ & Light brown. \\
\hline
\end{tabular}


Synopsis of the Reactions which are characteristic of the various Butter Colourers (Leeds).-Continued.

\begin{tabular}{|c|c|c|c|c|}
\hline Dye. & $\begin{array}{c}\text { Concentrated } \\
\mathrm{H}_{2} \mathrm{SO}_{4^{*}}\end{array}$ & $\begin{array}{c}\text { Concentrated } \\
\mathrm{HNO}_{3} .\end{array}$ & $\begin{array}{c}\mathrm{H}_{2} \mathrm{SO}_{4}+ \\
\mathrm{HNO}_{3} .\end{array}$ & $\begin{array}{c}\text { Concentrated } \\
\text { HCl. }\end{array}$ \\
\hline Marigolds ... & $\begin{array}{l}\text { Dark violet } \\
\text { green, perma- } \\
\text { nent. }\end{array}$ & $\begin{array}{l}\text { Blue, passes } \\
\text { at once into } \\
\text { dirty yellow- } \\
\text { ish green. }\end{array}$ & Green. & $\begin{array}{l}\text { Green to yel- } \\
\text { lowish green. }\end{array}$ \\
\hline $\begin{array}{l}\text { Safflower } \\
\text { yellow. }\end{array}$ & Light brown. & $\begin{array}{l}\text { Partially de- } \\
\text { colorised. }\end{array}$ & Decolorised. & No change. \\
\hline $\begin{array}{l}\text { Aniline } \\
\text { yellow. }\end{array}$ & Yellow. & Yellow. & Yellow. & Yellow. \\
\hline $\begin{array}{l}\text { Martius } \\
\text { yellow. }\end{array}$ & Pale yellow. & $\begin{array}{l}\text { Yellow, red- } \\
\text { dish precipi- } \\
\text { tate. }\end{array}$ & Yellow. & $\begin{array}{l}\text { Yellow pre- } \\
\text { cipitate, ex- } \\
\text { plodes on ig- } \\
\text { nition after } \\
\text { treat m en t } \\
\text { with } \mathrm{NH}_{3} \text {. }\end{array}$ \\
\hline $\begin{array}{l}\text { Victoria } \\
\text { yellow. }\end{array}$ & $\begin{array}{l}\text { Partially de- } \\
\text { colorised. }\end{array}$ & $\begin{array}{l}\text { Partially de- } \\
\text { colorised. }\end{array}$ & $\begin{array}{l}\text { Partially de- } \\
\text { colorised. }\end{array}$ & $\begin{array}{l}\text { The colour } \\
\text { returns on } \\
\text { neutralisation } \\
\text { with } \mathrm{NH}_{3} \text {. }\end{array}$ \\
\hline
\end{tabular}




\section{PART III-CHEESE}

\section{INTRODUCTORY.}

In practice cheese is made from milk, either by the action of rennet or of acids. The curd which is thus obtained differs in the two cases both in its chemical and in its physical character.

The theory which Hammarsten has suggested for the action of rennet on milk, mention of which has already been made on page 8 , is at the present time generally accepted. According to it the rennet splits up the casein of the milk into two new simpler substances-paracasein or cheese and whey albumin. Paracasein is regarded as being formerly combined with the lime of the casein, and as this new compound is insoluble in the milk serum, it separates out in the form of a coagulum, which does not fall to the bottom. This coagulum is the crude cheese, and has the property of elasticity, and the power under certain conditions of shrinking together very considerably, so that the whey is squeezed out. The whey albumin, which is only present in small quantities compared to the paracasein, is soluble, and remains in the whey.

When acid is used for the preparation of cheese the action is quite different. Generally the acid, which causes the coagulation, is lactic acid, which is obtained when the milk, either with or without the addition of sour buttermilk, becomes sour. Lactic acid acts like any other acid, namely, by forming a soluble calcium salt with the lime which is combined with the casein, and so the latter, which is thus freed, is precipitated. The curd obtained in this way is not elastic, but open and sticky, and contracts less than does rennet curd.

The crude cheese which has been obtained in one or other of the above-mentioned ways, and the rennet cheeses are far and away the most numerous, is then cut into small pieces by 
means of special tools. This causes the pieces of eurd to gradually contract and become smaller. During this part of the manufacture the temperature is often raised, more particularly with hard cheeses. After the curd has been taken out of the whey, it is put into a shape, and then either pressed to make hard cheese, or not pressed in the case of soft cheese. When the whey has flowed away more or less completely, the cheese is salted, either by laying it in brine or by strewing salt upon the surface. After these operations the cheese is left to ripen, and this takes place in very different ways and in varying times, the longest time being required for the hard cheeses. The process of ripening in cheese consists in the continued splitting up of the casein into substances of a simpler structure and greater solubility. In the case of hard cheeses the changes due to ripening proceed regularly through the mass, whilst in the soft varieties they proceed from the outside inwards towards the centre. These chemical changes are due both to the micro-organisms found in the mass of casein, and to enzymes present in the milk or rennet.

The majority of cheeses are made from cows' milk, only a few special sorts being made from sheeps' (Roquefort), goats', or other milk. Cheese is sometimes made from whole milk, to which cream has been added, whilst other varieties are made from whole milk or various proportions of whole or skim milk, in some few cases cheese being made from skin milk alone.

A distinction is also made between the cheeses with large holes, those with small holes, and those which are compact and practically without holes; the distinction, however, applies only to hard cheese.

Naturally there are a great number of intermediate stages between the chief types depending upon the working of the curd, the temperature at which the cheese is made, \&c. Further variations are introduced in pressing, salting, and in the temperature at which ripening takes place, as well as the point at which ripening is regarded as complete. With such a large number of varying factors it is possible to make from the original curd a large number of cheeses, differing more or less in appearance, taste, and smell from one another, but all of which form a valued and nourishing article of diet. 
The chemical composition of the many varieties of cheese is naturally very different. Where the cheese has been made from a rennet curd, the majority of the undissolved substances in the milk-fat, di-and tri-calcium phosphates, as well as the lime which is in combination with the casein-are carried down with the coagulated casein. This rennet curd has the same reaction as the milk, or it may be slightly alkaline. The sour milk or acid curd on the other hand contains no insoluble lime salts, because during the formation of the curd the lime salts pass into the soluble form, owing to the distinctly acid character of the medium. The difference in reaction between rennet and acid cheeses is of fundamental importance with regard to the ripening of the two varieties.

Owing to the less acid character of the rennet curd the various classes of micro-organism do not meet with such an adverse medium as is the case in the sour milk cheeses. A free development of one or more of the groups is therefore possible; and the way in which the cheese has been made, the pressing, the salting, and the storing, determine which of the microorganisms shall predominate. From rennet curd it is therefore possible to prepare cheeses with very varying properties and chemical composition. In the case of sour milk or acid curd the state of affairs is different for then the lactic acid bacteria predominate from the beginning. It is only possible also for those bacteria which can flourish in an acid medium to take part in the ripening of cheese of this class. This being so, ripening proceeds in a one-sided manner, and for that reason all sour milk cheeses are more or less alike.

Apart from the above-mentioned chief differences in the composition of rennet and sour milk cheese, a difference which begins with formation of the curd, there are also variations in the amount of fat which the mass of coagulated casein contains according to the richness of the milk.

During ripening soluble substances are gradually formed from the insoluble paracasein, and the original porcelain-like elastic curd, thereby becomes transformed by degrees into a yellowish, homogeneous, plastic mass the cut surface of which somewhat resembles butter.

At first substances which hold a position between paracasein and peptones, e.g. caseoglutin are formed, and afterwards, 
or at the same time, albumoses and peptones. Further changes result in the splitting up of these and the formation of amido-acids, such as phenylamidopropionic acid, leucine, tyrosine, glycocoll, as well as a large number of other compounds, e.g. alanine, amidovalerianic acid, pyrrolidine dicarboxylic acid, aspartic acid, glutamic acid, tryptophane, histidine, lysine, putrescine, cadaverine, guanidine, and succinic acid, finally ammonia also.

The process of cheese ripening cannot, as regards the changes in the paracasein, be looked upon as decomposition of the proteins, for those substances, e.g. indol, skatol and phenol, which are characteristically formed during such cleavages, are not found in ripening or ripe cheese.

Volitile fatty acids, such as caproic or butyric are formed during ripening owing to the splitting up of the fat. ${ }^{1}$

The milk sugar which gets into the cheese along with the whey undergoes lactic acid fermentation whilst the cheese is being made and pressed so that after a few days every trace of it has vanished from the fresh curd. The holes found in cheese are due, at least in Emmental cheese, according to von Frendenreich and Orla Jensen, ${ }^{2}$ to propionic acid formentation caused by certain bacteria. In this process the calcium lactate formed by the lactic acid bacteria, is transformed into propionic and acetic acids, and carbon dioxide, the last of which causes the formation of the holes.

$$
3 \mathrm{C}_{3} \mathrm{H}_{6} \mathrm{O}_{3}=2 \mathrm{C}_{3} \mathrm{H}_{6} \mathrm{O}_{2}+\mathrm{C}_{2} \mathrm{H}_{4} \mathrm{O}_{2}+\mathrm{CO}_{2}+\mathrm{H}_{2} \mathrm{O}
$$

The above mentioned substances are naturally not the only ones formed during the ripening of the cheese, but they may be appropriately regarded as the mile-stones on the road along which the curd passes whilst being broken down. It is not yet possible by chemical means to isolate and determine all the substances formed during the process of ripening so that for the present little more can be done than to follow the main chemical lines upon which the process is based. Bondzynski ${ }^{3}$ has introduced the idea of extent and depth in cheese ripening. By extent Bondzynski understands the quantity of curd which is converted

1 Orla Jensen, "Studier over de flygtige Syrer i Ost og Bidrag til Ostefermenternes Biologi." Dissert, Kopenhagen, 1904; Landw, Jalurbuch der Schweiz, 1904.

2 Landw. Jahrbuch der Schweiz, 1906,

3 Ibid., 1894, p. 189. 


\section{I84 MILK AND DAIRY PRODUCTS PART}

into a water-soluble form, and by depth, the degree to which the curd has been split up. If, therefore, the ripening process in a certain sort of cheese has considerable extent but only little depth, then that means that the greater part of the casein has passed into the soluble form, but that further changes have advanced only slightly. To make this clear attention is called to certain ripe varieties of cheese the analyses of which are due to Eckles and Rahn. ${ }^{1}$

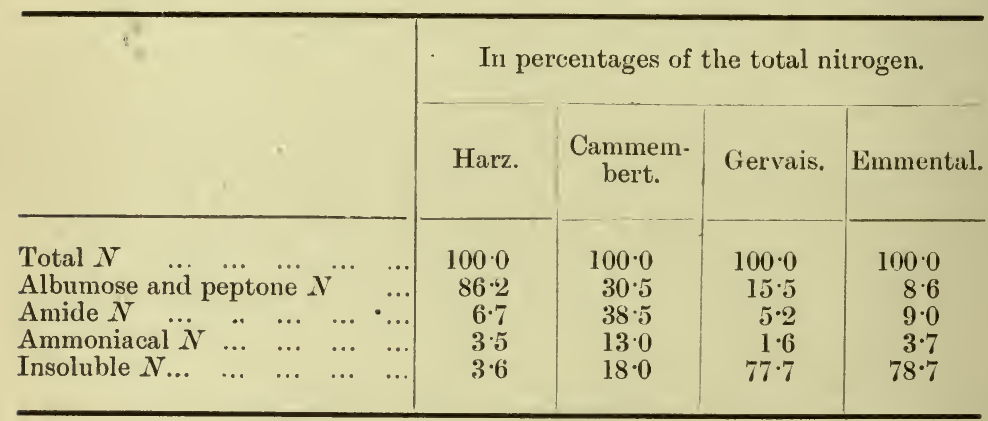

Therefore the ripe Harz Mountain cheese, for example, has the greatest extent of the four varieties, but the greatest depth is possessed by the Camembert. I. König gives the following average composition to the different kinds of cheese.

\begin{tabular}{|c|c|c|c|c|c|c|}
\hline & & & Water. & $\begin{array}{l}\text { Nitrogenous } \\
\text { substances. }\end{array}$ & Fat. & $\begin{array}{c}\text { Mineral } \\
\text { substances. }\end{array}$ \\
\hline $\begin{array}{l}\text { Cream cheese } \\
\text { Fat , } \\
\text { Half fat cheese } \\
\text { Skim milk ,, }\end{array}$ & $\begin{array}{l}\cdots \\
\cdots \\
\cdots \\
\cdots\end{array}$ & $\begin{array}{l}\cdots \\
\cdots \\
. . \\
\cdots\end{array}$ & $\begin{array}{c}\text { Per cent. } \\
36 \cdot 31 \\
38 \cdot 00 \\
39 \cdot 79 \\
46 \cdot 00\end{array}$ & $\begin{array}{c}\text { Per cent. } \\
18 \cdot 84 \\
25 \cdot 35 \\
2967 \\
34 \cdot 06\end{array}$ & $\begin{array}{c}\text { Per cent. } \\
40 \cdot 71 \\
30.25 \\
23.92 \\
11.65\end{array}$ & $\begin{array}{l}\text { Per cent. } \\
310 \\
4.97 \\
4 \cdot 73 \\
4.87\end{array}$ \\
\hline
\end{tabular}

The Analysis of Cheese.

Sampling.

Care should be observed when taking a sample of cheese for analysis to see that it represents the bulk. It is not

${ }^{1}$ Centralblatt fïr Bakt., Section 2, Vol. XIV, p. 676. 
sufficient to obtain a sample from the middle of the cheese, but parts from just below the surface should be taken also. In the case of hard cheeses and large soft cheeses a wedge-shaped piece should be cut in a radial direction through the cheese so as to include parts both of the interior and of the outside portion. Small cheeses are best used whole for analysis. If the sample has to be forwarded to the laboratory, it should be either put into a tightly closing glass bottle, or if this is not possible it should be wrapped in tin foil or in parchment paper.

When the sample comes to be analysed it is best in the case of hard cheese to first cut away the crust and then grind the remainder in an iron mortar. The ground cheese ought then to be well mixed with a horn spoon and a sample taken for analysis. Where a soft cheese is being examined the sample (or the whole cheese in the small varieties) is ground in a porcelain mortar until a completely homogeneous mass is obtained.

\section{Determination of the Percentage of Water.}

Orla Jensen and Ernst Plattner ${ }^{1}$ advise the following method. In the case of hard cheeses, 3-5 g. of ground cheese are dried in a vacuum over sulphuric acid for a few days and then completely desiccated in the water-oven. If the cheese were heated at the beginning it would melt to a horny mass which would obstinately retain the last traces of water. The smaller the amount of water in the cheese when it is heated, the less will the paracasein turn brown, and the smaller will be the loss of volatile fatty acids. It is impossible, however, to avoid loss of ammonia, and if the drying in the water-oven extends over a long time, all the ammonia may be driven off. Clearly, then, the drying of the cheese until a constant weight is obtained is impracticable, so that after the preliminary drying in vacuum it is sufficient to heat for 8 hours in the water-oven and presume that all the water has been got rid of.

Soft cheese must be mixed with a considerable quantity of sand before the drying begins. A small porcelain dish on which

1 "Bertrag zur Kïseanalyse," Laudw. Jahrbuch der Schweiz, 1906. 
has been placed a layer of sand, $1 \mathrm{~cm}$. high, and a short glass rod is carefully dried and weighed, and then a little of the ground cheese is placed on the sand, weighed, heated to melting point on the water-bath and well stirred up with the sand. The subsequent treatment is the same as with hard cheese.

\section{Determination of Fat. \\ a. Scientific Methods.}

\section{The Extraction Mcthod.}

About $5 \mathrm{~g}$. of cheese, if the sample is rich in fat, otherwise 10 g., are weighed out and brought into a porcelain mortar containing about an equal quantity of fine ignited sand, and the whole then dried in the manner described above. After drying, the contents of the mortar are brought into a fat-free extraction cartridge, which is placed in a Soxhlet extraction apparatus. The mortar is then washed out several times with ether, which is poured over the cartridge in the extractor, and the extraction carried out in the usual way for several hours. When the extraction has been going for about 2 or 3 hours it is interrupted, and the cartridge taken out, and the contents ground in a mortar. The extraction is then continued and followed after a few hours by a further grinding up until the extraction flask after evaporation of the ether shows no increase in weight.

This method is tedious and takes a long time; it is also only suitable for whole milk cheeses, or such cheeses as contain a fair quantity of fat. With the poorer cheeses, and particularly with skim milk cheese, the results are far too low. This is partly due to the inability of the ether to reach the small fat globules which are embedded in the dry cheese, and probably also to a cause pointed out by Orla Jensen, namely, that some of the free fatty acids of the cheese are present as ammonia salts and so escape extraction by the ether. It is therefore preferable, and for skim milk cheese essential, to make use of one of the methods now to be described. 


\section{The Schmid-Bondzynsli Mcthod. ${ }^{1}$}

This method, which was originally designed for milk, has only found application to any extent in the estimation of fat in cheese. Ratzlaff"s ${ }^{2}$ modification, which is a combination of the Schmid-Bondzynski and the Röse-Gottlieb methods, is preferable to the original.

Three to five grams of cheese are weighed into a 30 c.c. flask and 10 c.c. of $\mathrm{HCl}$ (sp. gr. 1.125) added. The flask is then warmed on an asbestos plate over the free flame until the cheese dissolves, and afterwards for 8-10 minutes at boiling point. After cooling, the solution, which now ought to have a reddish-brown colour with a layer of fat on the surface, is transferred to a Gottlieb cylinder which is graduated the whole length. The flask is washed with ether from a small wash-bottle, and the washings transferred to the cylinder, 25 c.c. of ether being used in all. The cylinder is corked up, well mixed, and then 25 c.c. of petroleum ether (b. p. below $70^{\circ}$ C.) added. After the cylinder has been again well shaken, it must be allowed to stand for at least two hours, and in that time the ethereal solution separates sharply from the cloudy layer below, so that it can easily be syphoned off into a tared flask, leaving only 1.5-2 c.c. behind. The ether may then be evaporated, as Ratzlaff advises, the fat weighed, and the total amount calculated from the proportion of the whole of the ethereal solution to that part which was syphoned off. To do away with this calculation, Siegfeld ${ }^{3}$ adds another 25 c.c. ether and petroleum, shakes up again, and then syphons off the ethereal solution, when it has separated, into the same flask, and this gives the total fat in the cheese.

\section{Röse-Gottlieb Method.}

This excellent method for the determination of fat has recently been modified by Palmquist, ${ }^{4}$ and made quite suitable for the estimation of fat in cheese.

About 1 gram of cheese, which has been finely divided and

1 Chemisches Centralblatt, Vol. I, p. 447.

2 Milch-Zeitung, 1903.

3 Milch-Zeitung, 1904, No. 19.

4 Svensk. Kem. Tidskr., 1905, p. 58. 
well mixed in the usual way, is weighed into an ordinary Gottlieb tube without foot (Palmquist describes a special form of tube with a bulb-like extension at the lower end, but the ordinary tubes are just as suitable). The cheese is then treated with 10 c.c. of 2.5 per cent. ammonia, and, by warming on the waterbath and shaking, a milky homogeneous solution is obtained, to which, after cooling, 10 c.c. of alcohol are added. After shaking, 25 c.c. of ether are added and the tube again shaken, after which 25 c.c. of petroleum ether are introduced. The tube must again be well shaken and inverted before being left to stand. Two sharply defined layers separate out, as in the case of milk, and after a few hours the clear ethereal solution of fat is syphoned off as completely as possible into a tared flask, care being taken that none of the cloudy lower layer passes over. A further 25 c.c. ether and petroleum ether are then added, the contents well mixed after each addition, and finally left to separate out. The ethereal solution is syphoned off into the the same flask as before, the ether evaporated off, and the fat dried and weighed as usual. ${ }^{1}$

This method gives most excellent results even with skim millk cheese, and is undoubtedly the best known method for the determination of the fat in cheese; it is also very simple and easy to carry out.

M. Weibull ${ }^{2}$ has recently improved Palmquist's modification of the Gottlieb method, so that it is now almost identical with that used for milk, which must be regarded as a great advantage. The cheese is finely ground up, and $1.03 \mathrm{~g}$. are placed in the bottom of a Gottlieb tube and 10 c.c. of ammonia (the strength is of no importance) added. By shaking the tube and gradually warming it in a water-bath to a temperature of $75^{\circ} \mathrm{C}$., the cheese is brought into solution. If this is not accomplished in half an hour, 10 c.c. of 95 per cent. alcohol are added, and the liquid again well shaken and allowed to stand at $75^{\circ} \mathrm{C}$. In ordinary cases when the temperature has reached $75^{\circ} \mathrm{C}$., the tube can be allowed to cool, and then 10 c.c. of alcohol added and the ordinary Gottlieb method followed. When syphoning off, 1.5 c.c. of the clear ethereal solution is allowed to remain

1 Palmquist only takes an aliquot part of the ether-fat solution, and calculates from it the weight of the total fat contained in the cheese. In the above method all the fat is obtained together in the flask.

2 Zeitschrift für Unt. d. Nahr. und Genussmittel, 1906. Ibid., 1909. 
behind, and the weight of fat left in the flask after the evaporation of the ether-benzene is multiplied by 100, which gives the percentage amount of fat in the sample of cheese.

\section{(b) Practical Methods.}

\section{Gerber's Test.}

Gerber's acid butyrometer test (p. 69) can also be used for the determination of fat in cheese when the results do not require to be scientifically correct. The method worked out by Gerber is not as good as the modification proposed by Siegfeld, ${ }^{1}$ so the latter only will be described here.

About 2.5 grams of cheese are weighed out into a small flask and dissolved in 10 c.c. of sulphuric acid (sp. gr. 1.5) by warming over a naked flame and shaking. Solution having been effected, the contents of the flask are transferred by means of a small funnel, the lower end of which is cut off obliquely, into a Gerber tube. The flask and funnel are washed out with small quantities of the same acid until the volume of the liquid is 21 c.c. It is advisable to have a special mark on the butyrometer tube for this purpose. One c.c. of amyl alcohol is then added, the tube shaken and warmed in a water-bath to $60^{\circ}-70^{\circ} \mathrm{C}$., again shaken and finally whirled in the centrifuge for 5-6 minutes. When the tube is taken from the centrifuge it is again placed in the waterbath, after which the column of fat can easily be read off. A simple calculation is necessary, for the scale of the milk butyrometer is graduated for 11 c.c. milk, which is equivalent to $11.33 \mathrm{~g}$., if the specific gravity of milk be taken as 1.03. All that is required is to multiply the percentage of fat by 11.33 and divide by the weight of cheese taken. This is expressed by the formula:

$$
f=\frac{p \cdot 11 \cdot 33}{k}
$$

where $f=$ the percentage of fat in the cheese,

$p=$ the percentage of fat read off,

$k_{i}=$ the weight of cheese taken.

With skim milk cheese Gerber's method gives unreliable 
results, but with the richer sorts it is sufficiently correct to be used when a very high degree of accuracy is not needed.

\section{Determination of the Nitrogenous Substances.}

(a) Estimation of the total Nitrogen.

Two grams of cheese are analysed by Kjeldahl's method (p. 80).

(b) Estimation of the Water-Soluble Nitrogenous Compounds.

Ten grams of cheese are rubbed up to a thin paste with water at $40^{\circ} \mathrm{C}$., and then transferred to a 250 c.c. graduated flask, which is filled to the mark. The flask is allowed to stand for twentyfour hours at room temperature, and during that time it is frequently shaken. A few drops of formalin should be added to prevent further decomposition. By evaporating 25 c.c. of the filtrate the total water-soluble nitrogenous compounds are obtained, and can be analysed also by Kjeldahl's method. In another portion of the filtrate, 50 c.c., the soluble protein can be determined by making acid with 30 c.c. of dilute sulphuric acid (1:4) and precipitating with 20 c.c. of 10 per cent. phosphotungstic acid. After standing for twenty-four hours the precipitate is collected on a filter, washed, and treated according to Kjeldahl's method for the determination of nitrogen.

'The phospho-tungstic acid not only precipitates the protein but also any basic decomposition products, and these have to be determined in another portion of the original filtrate by distillation with magnesia, and deduction of the nitrogen so found from that obtained with the phospho-tungstic acid. The watersoluble nitrogen minus the soluble protein and the ammonical nitrogen, is termed the amide nitrogen.

\section{Determination of the Ash.}

This is done in the ordinary way by carefully igniting the cheese in a platinum basin. If the common salt has to be specially estimated, then the ash is dissolved in water and an aliquot portion titrated with $N / 10$ silver nitrate solution potassium chromate serving as an indicator. 


\section{B. Detection of Anulteration.}

Cheese is not infrequently adulterated by replacing the fat in the milk from which the cheese is made by other less valuable fats in the form of an emulsion. If, therefore, the fat of a cheese has to be more closely investigated, the first thing is to obtain a considerable quantity of it. The best way to achieve this is by the method described by H. Brehmer. ${ }^{1}$

One hundred grams of finely divided cheese are ground in a mortar with acidulated water (five parts dilute sulphuric acid to 200 parts water) at a temperature of $20^{\circ}-30^{\circ} \mathrm{C}$., the water being added gradually. The emulsion is then centrifuged, and the fat which separates is kneaded with a little water, and then melted at a moderately low temperature. After filtration through a dry filter it is then ready for use. The methods used in investigating the fat are naturally the same as those which have been described for butter-fat.

It is possible to extract the fat from the cheese by simply warming it in a drying oven at about $80^{\circ} \mathrm{C}$., but there is always the danger that if the temperature rises above this point the fat begins to decompose. As regards the margarine test in the case of the fat of cheese, the method described on page 167 is followed exactly. As, however, the prescribed addition of sesamé oil in the case of margarine cheese is only 5 per cent., more of the fat, 10 c.c., should be taken for the test.

\section{Testing of Rennet.}

Rennet is the enzyme extracted from the mucous membrane of the stomach of mammals, and small amounts of it have the power of curdling or coagulating large quantities of milk. This process of coagulation only takes place within a certain range of temperature, the most suitable point being slightly above $40^{\circ} \mathrm{C}$. This enzyme (scientifically named chymosin) is found in large quantities in the stomachs of young animals living solely upon milk, and a typical case is that of the calf. From the earliest times man has obtained the rennet which he has used in the making of cheese from milk, by extracting

${ }^{1}$ Forschungsbericht über Lebensmittel, u.s.vv., 1897, p. 51. 
dried calves' stomachs with water, some preservative generally being also added.

Soxhlet ${ }^{1}$ gives the following directions for the preparation of rennet. The fresh stomachs inust first be carefully emptied blown out, dried in the air, and then kept for at least three months. After that part of the stomach which contains no folds has been removed, the rest is cut into pieces about one square centimetre in size. To each 100 of g. stomach, 1 litre water, $50 \mathrm{~g}$. of common salt, and $40 \mathrm{~g}$. of boracic acid are then added $_{2}$ "and the whole left to stand for five days at room temperature. Then $50 \mathrm{~g}$. more salt are added for each litre of water, and the fluid filtered off. From 1 litre of water there is obtained about 800 c.c. of filtrate, and this is made up to a litre by the addition of 200 c.c. of 10 per cent. salt solution to which boracic acid has been added to saturation. Such a solution of rennet acts in the proportion of $1: 10,000$, and keeps good for a long time. Nowadays it is seldom that the cheesemaker prepares his own rennet, for there are so many good preparations to be bought at a moderate price. These rennet preparations are sold both in liquid and powder form, or in tablets. The manufacture of the last two preparations is a trade secret, but they are composed largely of common salt, often as much as 95 per cent.

A commercial rennet solution ought to be quite clear and without any unpleasant smell, and if kept for a year should not lose more than 25 per cent of its original solution. After being keptin the dark for several months it ought to still act to the extent of 1:6000. Good rennet powder ought to be almost colourless and odourless, and nearly completely soluble in water. Rennet powder is naturally much more active than liquid rennet, some of the powders which are sold having a strength of $1: 300,000$ or even stronger.

To measure the strength of a given rennet preparation it is usual to follow Soxhlet's proposal and estimate, how many c.c. of milk are coagulated in 40 minutes at a temperature of $35^{\circ} \mathrm{C}$. by 1 c.c. of liquid rennet or $1 \mathrm{~g}$. of rennet powder. Fleischmann ${ }^{2}$ has worked out the following details

1 Milch-Zeitung, 1877, p. 497. 1907.

2 Fleischmann, Lehrbuch der Milchwirtschaft. Fourth edition. Leipsic, 
for the test. Five c.c. of the rennet solution, or $5 \mathrm{~g}$. of the rennet powder are made up to 100 c.c. with distilled water. After well mixing, 10 c.c., equivalent to 0.5 c.c. or $0.5 \mathrm{~g}$. of the rennet preparation, are drawn off with a pipette and run into 500 c.c. of perfectly fresh milk, warmed to $35^{\circ} \mathrm{C}$. and the exact time, to a second, noted when the two liquids are brought together. The solution of rennet is blown with force into the milk so that it is uniformly distributed and then the whole is well and quickly shaken round. As will easily be seen there is now one part of the rennet preparation-1 c.c. or 1 g. - to each 1000 c.c. of milk. A thermometer which has previously been placed in the milk is moved gently to and fro, and the length of time which elapses before coagulation is apparent is noted. This point is indicated by the appearance of fine particles of milk behind the thermometer. The temperature of the milk must be kept as nearly as possible at $35^{\circ} \mathrm{C}$., during the determination. If, for example, the time required for coagulation is found to 5.55 minutes, the quantity of milk $(x)$ which would be coagulated in 40 minutes at the same temperature by a similar quantity of rennet would be:

$$
\begin{aligned}
5 \cdot 55: 40 & =1,000: x \\
x & =7,207
\end{aligned}
$$

The rennet preparation thus tested is found to have a strength of $1: 7,207$ or in round numbers $1: 7,200$.

Rennet powders which act more energetically are tested by taking $1 \mathrm{~g}$., dissolving it in 200 c.c. of water, allowing it to stand for at least a quarter of an hour, and then, after well stirring up, 5 or 10 c.c., according to circumstances, are allowed to act upon $500 \mathrm{~g}$. of new milk at $35^{\circ} \mathrm{C}$. The proportion of rennet to milk ought to be either nearly $1: 20,000$ or $1: 10,000$. If for instance the proportion of rennet to milk had been $1: 20,000$ and the coagulation period again 5.55 minutes (5 minutes, 55 seconds) then the quantity of milk $x$ which would be coagulated at the same temperature in 40 minutes by 1 part of rennet would be,

$$
\begin{aligned}
5 \cdot 55: 40 & =20,000: x \\
x & =144,144
\end{aligned}
$$

or in other words, the strength of the rennet powder is in round numbers $1: 144,000$. This method of testing rennet 
is the most usual and is quite sufficient for ordinary practical purposes. Devarda ${ }^{1}$ has devised a more exact method which can be used if more accurate determinations are to be made. Devarda's method is based upon the comparison of the sample of rennet with a "standard rennet" prepared according to a definite formula and is carried out as follows.

A commercial rennet powder of medium strength is used as the standard rennet. It must be homogeneous and dry, and should only contain a small quantity of mineral salts, and ought also to give a clear solution with water. After being kept in the dark for one or two years it ought not to have diminished in strength even to a slight degree. To get a standard rennet of constant strength Devarda prepared a mixture of the commercial rennet powder with fine sugar. This preparation coagulated milk in the proportion of $1: 100,000$ in 6 minutes. The rennet solution must be prepared immediately before each test.

To determine the strength of the standard rennet, it is absolutely essential to have a perfectly normal milk got from a herd of cows fed on a standard ration. An average sample of 1 litre of the milk is drawn and cooled at once to $15^{\circ} \mathrm{C}$. Before using this milk for testing the rennet, it must first of all be thoroughly shaken to free it from carbon dioxide. Milk that is a few hours old must not be used for this purpose. The average number 12 is taken as the first value for the strength of the standard rennet when the above test is made with normal milk under the conditions given above. When the strength of the standard has been determined once for all, it can be used as a measure of the strength of an unknown rennet preparation. The procedure when determining the strength is as follows:

Two hundred c.c. of fresh normal cows' milk are poured into a 300 c.c. glass flask and warmed to $35^{\circ}$ C. To this 2 c.c. of rennet solution are added, the time being carefully noted, preferably on a seconds watch with stop action, and the contents of the flask gently shaken. A thermometer is then sunk into the milk, and the flask placed in a water-bath having a temperature of $36^{\circ} \mathrm{C}$. Whilst the flask is slowly moved to and fro, the appearance of the milk as it flows from the sides of the flask is

\footnotetext{
${ }^{1}$ Landwirtschaftliche Versuchsstationen, 1896, Vol. XLVII, p. 401.
} 
noticed, and when a point is reached at which the milk becomes thick and cheesey (which always happens suddenly), the exact time in seconds is noted. During the whole of the time of the experiment the milk must have a constant temperature of $35^{\circ} \mathrm{C}$.

The solutions of rennet which are to be used for such tests must have the following strengths: $(a)$ In the case of rennet liquids, 10 c.c. are diluted to 200 c.c. with water. (b) Rennet powders, $1.25 \mathrm{~g}$. are dissolved in 200 c.c. water.

The strength is obtained by aid of the following formulie:

With liquid rennet,

With rennet powder,

$$
W=\frac{80 \cdot 000}{t}
$$

$$
W=\frac{640 \cdot 000}{t} .
$$

If the milk used for such determination is quite pure, normal and fresh, the strengths of the standard rennet and of the rennet being tested are directly comparable and proportional. Where the milk is not absolutely in accordance with the above requirements, and this must be regarded as the usual condition, it must be sterilised for from half to three-quarters of an hour at $75^{\circ}-80^{\circ} \mathrm{C}$. in order to permit of the two solutions of rennet being compared with one another. If then the strength of the standard rennet and of the rennet which is being tested have been ascertained in the manner described above, the real strength of the latter may be calculated in the following manner:

With rennet solutions,

With rennet powders,

$$
T V_{1}=W \frac{t}{8 t_{1}}
$$

where

$$
W_{1}=W_{\frac{t}{t}}^{t}
$$

$W=$ strength of standard rennet with normal milk.

$W_{1}=\quad " \quad$ "rennet being treated with normal milk.

$t=$ time of coagulation of abnormal milk with standard rennet.

$t_{1}=$ ditto with the rennet being tested. 


\section{PART IV_PRESERVED MILK AND OTHER MILK PREPARATIONS}

\section{INTRODUCTORY.}

UNDER the heading of preserved milk may be elassed eertain preparations of milk which are prepared so as to enable the milk to be kept for a longer time than would be otherwise possible.

These different preparations eontain the whole of the constituents of milk, but sometimes substances are added to increase the keeping properties. In some cases the preserved milk eontains all the water normally present in milk, whilst in other eases it is partially or almost entirely driven off.

The usual preparations of preserved milk are sterilised milk (pasteurised milk ean to a certain extent be ineluded), Buddised milk, condensed milk, dried milk or milk powder (also milk tablets).

By sterilised milk is understood milk which, by heating, has been completely freed from micro-organisms. It is, however, difficult to obtain absolutely sterile milk, for various sporeforming bacteria have very resistant spores which are able to withstand long-continued heating at fairly high temperatures. If the milk is to be sterilised in one operation, it must be heated (usually in hermetically closed glass bottles) by steam under pressure in a strong-walled apparatus. Nevertheless, a temperature of $120^{\circ} \mathrm{C}$. for 2 hours is necessary if the milk is to be rendered absolutely sterile, and if the temperature is only about $100^{\circ} \mathrm{C}$., a eonsiderably longer time is required. The milk, however, undergoes such great changes in taste, appearance, and composition as to be unsuitable for food. In practice, therefore, the heating is only for a shorter time and the temperature is kept fairly low, say 20 minutes at $112^{\circ} \mathrm{C}$. Where the temperature only slightly exceeds $100^{\circ} \mathrm{C}$., a longer time is necessary. In this way the milk, apart from a boiled 
taste, shows no other signs of having been heated, but it is far from being absolutely sterile. All the vegetative forms of bacterial life, together with the less resistant spores, are killed, but some of the most resistant spores, particularly the so-called peptonising bacteria, remain alive, although they develop only very slowly when the milk, after "sterilisation," is kept at a low temperature. Such milk will keep for a considerable length of time, although it may not be actually sterile. Absolutely sterile milk cannot be obtained commercially. 'The only way to obtain a sterile milk (unless it is heated for a long time at a high temperature, which, as already seen, renders it unfit for use) is to subject it to fractional sterilisation as recommended by Tyndall. For this purpose, the milk is heated on consecutive days for 2 hours at $65^{\circ}-70^{\circ} \mathrm{C}$.; in the interval the temperature is kept between $25^{\circ}$ and $55^{\circ} \mathrm{C}$. By this means the spores which remain alive grow daily into fresh bacteria, which are then killed on the following day by the higher temperature, and so the milk finally becomes sterile. The taste and outward appearance of the milk hardly undergo any change by the treatment, but sterilisation in this way is very costly and tedious, and has not found any considerable application.

Even at $100^{\circ} \mathrm{C}$, and still more above that temperature, the properties of milk are altered, although, judging by the appearance, the milk is unchanged. The most important of these changes are the following:

The greater part of the albumen coagulates at about $70^{\circ} \mathrm{C}$. and the casein is changed at a temperature above $100^{\circ} \mathrm{C}$., so that the milk acquires a brownish tinge, and this alteration in colour is therefore not always due to the caramelising of the milk sugar.

The acidity of milk decreases on heating to $100^{\circ} \mathrm{C}$., because of the separation of carbon dioxide. When the temperature exceeds $100^{\circ} \mathrm{C}$. the acidity increases, owing to the splitting off of phosphorus-containing acids (nucleic acid) from the casein. The loss of carbon dioxide on heating leads also to the precipitation of lime salts (phosphates and citrates). Milk becomes less susceptible to the coagulating action of rennet the higher the temperature rises, until finally coagulation ceases if the milk has been heated for half an hour at $110^{\circ} \mathrm{C}$.

The enzymes of milk are in general destroyed by moment- 
arily heating it at $80^{\circ} \mathrm{C}$. Lecithin is partly destroyed when the milk is heated. ${ }^{1}$ Milk sugar suffers practically no change from such temperatures as are met with in practice.

When milk is heated the fat tends to flow together into visible drops, which, on standing, impart an unpleasant appearance and a thick consistency to the layer of cream. Such a layer does not easily mix again with the milk. Latterly this drawback has been removed by "homogenising" the milk, that is, subjecting it to energetic mechanical treatment. The process devised by A. Gaulin is most convenient for this purpose. The milk is heated to $85^{\circ} \mathrm{C}$., and then forced through fine metal capillary tubes under a pressure of 250 atmospheres against a conical surface of agate. The fat globules of the milk are then split up into extremely fine particles, so fine, in fact, that when the milk stands they are no longer able to rise to the surface and form cream. Homogenised milk cannot either be separated by means of a separator. Thus the inconvenience due to the fat globules running together is prevented, and the unpleasant deposit of particles of cream in the neck of the bottle no longer takes place.

In the pasteurising of milk the heating is for a longer or shorter time at a temperature below $100^{\circ} \mathrm{C}$. Generally the temperature varies between $65^{\circ}$ and $90^{\circ} \mathrm{C}$., and the time from several hours to a few minutes. The object of the pasteurisation is to obtain better keeping properties, and to destroy any pathogenic germs, for these are, in general, easily destroyed by heat, except the anthrax bacillus, which, owing to its ability to form spores, is very resistant. Pathogenic bacteria which accidentally find their way into milk are killed at $80^{\circ} \mathrm{C}$. where the sample is normal.

Milk is pasteurised either by heating it in glass bottles in a water-bath, or allowing it to flow through a continuously working pasteurising plant, such as is usually found in large dairies. In the first case the time can be as long as desired, but in the latter case each particle of milk is only heated for a very few minutes.

Pasteurised milk usually keeps much better than unpasteurised, if, directly after pasteurising, the milk is conled to as low

1 Bordas et de Rackowski, Compt. rend de l'Acad. des Sciences, Vol. CXXXVI, p. 56. 
a temperature as possible, and then kept cool until required for use. Pasteurised milk does not, however, keep as well as does milk that has been almost sterilised, for in the former there remain many more resistant micro-organisms than when the heating has been carried above $100^{\circ} \mathrm{C}$.

A very grave danger lies in the use of pasteurised and partially sterilised milk, particularly for infants, if, as often happens, the heated milk after cooling is not kept at a low temperature so as to prevent the spores forming new cells. If such milk is kept for more than twenty-four hours at room temperature, particularly in summer, before being consumed, it often happens that the bacteria which escape the heating develop to a considerable extent, and Flugge ${ }^{1}$ has shown that the decomposition products that arise from their action on the protein of the milk are often of a poisonous nature, and can be a source of serious danger to infants. The worst of it is that decomposition may have gone very far before the appearance of the milk arouses suspicion.

The purely physical and chemical changes which, it has been seen above, take place when milk is heated to a high temperature, are also found, although to a less degree, as soon as pasteurisation exceeds $70^{\circ} \mathrm{C}$.

Buddised milk is the preparation invented by the Danish engineer, Budde. The principle of the method is to allow small amounts of hydrogen peroxide to act upon milk at a temperature of $50^{\circ}-55^{\circ}$. By the action of the peroxydases in the milk, the oxygen of the hydrogen peroxide is rendered active, and destroys bacterial life, so that a milk is obtained which is either sterile, if sufficient hydrogen peroxide has been used, or partially sterile if less has been added. The result in either case is to obtain milk which keeps better. It has been proved that the action of hydrogen peroxide on milk is greater at about $30^{\circ}$ than at ordinary temperatures, so that a smaller quantity of the peroxide is needed when the temperature is higher. Budde advises an addition of $0.35 \mathrm{~g}$. of hydrogen peroxide per litre, and the heating to be for at least three hours at $50^{\circ}-55^{\circ} \mathrm{C}$. This temperature is not high enough to bring about any of the chemical or physical changes which take place in partially sterilised or highly pasteurised milk.

1 Zeitschrift für Hygiene, 1894, Vol. XVII, p. 272. 
Buddised milk has not come into general use, and the reason may be that it is difficult to gauge the right amount of hydrogen peroxide to be added. Different milks possess very varying powers of decomposing hydrogen peroxide, no doubt due to the amount of catalase which they contain. If more hydrogen pcroxide is added than can be decomposed, the milk acquires a characteristically unpleasant taste, whilst a deficiency only results in a shorter or longer "stupefaction" of the bacteria. After some hours they become active again and spoil the milk. Further, also, when too little hydrogen peroxide has been added, the lactic acid bacteria may have been killed, but not the peptonising and putrefaction organisms, so that in the consumption of the milk there is the same danger as was seen to exist with pasteurised milk (p. 199).

It must also be clearly noted that the addition of any preservative to milk, even when of such a harmless nature as hydrogen peroxide, which splits up into water and oxygen, acts in opposition to the efforts which are now so strenuously and properly being made to ensure a supply of milk that shall be as free as possible from bacteria. The only preservatives that ought to be allowed are a high degree of cleanliness and a rapid and thorough cooling of the milk. If such preservatives as formalin and hydrogen peroxide are put into the hands of milk producers and milk dealers, the proper hygienic treatment of the milk during and after milking will be very liable to be neglected.

Condensed milk was first prepared by the American, Gail Borden, in 1856. The principle of the preparation has hardly undergone any change since then, and briefly is as follows :

Good fresh milk (considerable importance is attached to the quality of the milk) is evaporated down in a vacuum pan, similar to those used in a sugar factory, at a temperature of $40^{\circ}-50^{\circ} \mathrm{C}$. until the volume is reduced to a third or a quarter of the original. The evaporation must be conducted with great care, for otherwise the milk sugar crystallises out and this causes the condensed milk to feel "sandy" on the tongue. Condensed milk is made either with or without the addition of cane sugar; when it is added about 10-12 per cent. of the weight of the milk is taken. When the evaporation of the milk is at an end the yellowish-white syrup is sealed up in tins which 
usually hold about $450 \mathrm{~g}$. (1 lb.), and this quantity is equivalent to $1 \frac{1}{2}$ litres ( $2 \frac{1}{2}$ pints) of ordinary milk. 'The addition of cane sugar renders the milk very durable, and it may be kept for an indefinite period if the tin is not opened. Even when the tin has been opened the contents will remain good for about a week, and in some cases a little longer.

Milk prepared from condensed milk to which cane sugar has been added has a very sweet taste. Condensed milk is usually mixed with 3-4 times its volume of water, and this mixture has all the properties of ordinary milk.

Fleischmann, ${ }^{1}$ who has compared a very large number of analyses of condensed milk, gives the following composition to the article prepared with sugar:

\begin{tabular}{|c|c|c|c|c|c|c|c|}
\hline \multirow{6}{*}{$\begin{array}{l}\text { Water } \\
\text { Fat ... } \\
\text { Protein } \\
\text { Milk and } \\
\text { Crude as }\end{array}$} & & & $\therefore$ & \multicolumn{2}{|c|}{$\begin{array}{l}\text { Mean. } \\
25.686 \text { per cel }\end{array}$} & \multicolumn{2}{|c|}{ Fluctuations. } \\
\hline & $\ldots$ & ... & $\ldots$ & $10 \cdot 985$ & , & $7 \cdot 54-18 \cdot 78$ & , \\
\hline & $\ldots$. & $\ldots$ & $\ldots$ & $12 \cdot 325$ & ," & $7 \cdot 79-20 \cdot 14$ & ", \\
\hline & ne sug & gars & $\ldots$ & $48 \cdot 662$ & , & $41 \cdot 25-53 \cdot 89$ & , \\
\hline & $\ldots$. & ... & $\ldots$ & $2 \cdot 342$ & , & $1 \cdot 56-3 \cdot 87$ & ," \\
\hline & & & & 0.00 & & & \\
\hline
\end{tabular}

The proportion between the quantities of milk sugar and of cane sugar is generally:

\begin{tabular}{lllllll} 
& & \multicolumn{3}{c}{ Mean. } & Fluctuations. \\
Milk sugar & $\ldots$ & $\ldots$ & $\ldots$ & $16 \cdot 288$ per cent. & $10 \cdot 82-18 \cdot 35$ per cent. \\
Cane sugar & $\ldots$ & $\ldots$ & $\ldots$ & $32 \cdot 374,$, & $24 \cdot 11-40 \cdot 48$,
\end{tabular}

Condensed milk to which sugar has been added has an average specific gravity of 1.2820 at $15^{\circ} \mathrm{C}$., but it can fluctuate between $1 \cdot 254$ and $1 \cdot 4038$. D. Sidersky ${ }^{2}$ gives the following average analyses of different brands of sweetened condensed milk.

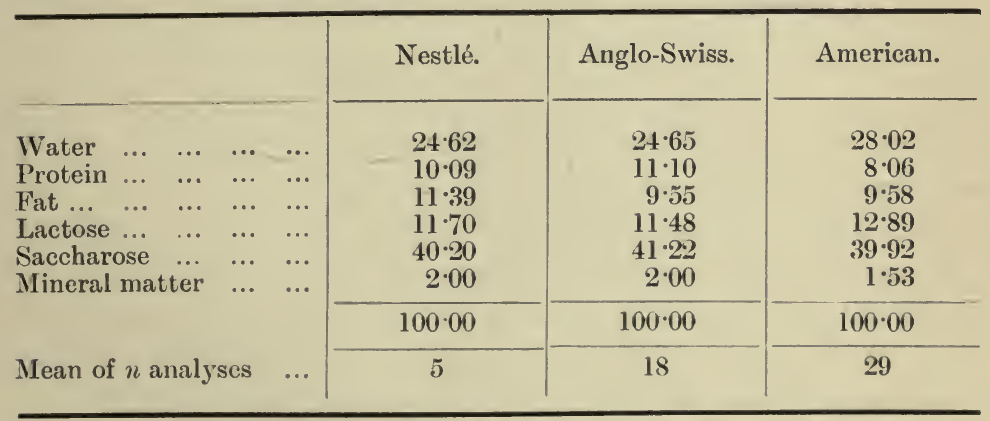

1 Lehrbuch der Milchwirtschaft. Fourth edition. Leipsic, 1907, p. 398.

2 Rapport au II' Congrès international de Laiterie à Paris, 1905. 
To avoid what, for some people, is the unpleasantly sweet taste of condensed milk, it is sometimes prepared without sugar, but then the condensed milk must be heated in the tins and these afterwards sealed hermetically to render the contents durable. The tins are therefore sterilised at $120^{\circ} \mathrm{C}$., and afterwards the sterilisation controlled by keeping them for some time at $30^{\circ}-40^{\circ}$ in the thermostat, so that if fermentation sets in, it is shown by the bulging of the tins.

When a tin of unsweetened condensed milk is opened, the contents do not keep as well as where sugar has been added, owing to the larger quantity of water in the former. Fleischmann gives the following composition to the unsweetened milk:

\begin{tabular}{|c|c|c|c|c|c|}
\hline \multirow{5}{*}{$\begin{array}{l}\text { Water ... } \\
\text { Fat... ... } \\
\text { Protein ... } \\
\text { Milk sugar } \\
\text { Crude ash }\end{array}$} & \multicolumn{3}{|c|}{ Mean. } & \multicolumn{2}{|c|}{$\begin{array}{l}\text { Fluctuations. } \\
46.40-53.54 \text { per cent. }\end{array}$} \\
\hline & $\cdots$ & $15 \cdot 668$ & , & $13 \cdot 12-19 \cdot 80$ & \\
\hline & $\ldots$ & $17 \cdot 806$ &, & $13 \cdot 61-26 \cdot 50$ & , \\
\hline & $\ldots$ & $15 \cdot 403$ & $"$ & $12 \cdot 50-17 \cdot 75$ & ", \\
\hline & $\cdots$ & $2 \cdot 528$ & , & $2 \cdot 00-2 \cdot 96$ & , \\
\hline & & & & & \\
\hline
\end{tabular}

The average specific gravity at $15^{\circ} \mathrm{C}$. is $1 \cdot 136$. Unsweetened condensed milk was introduced some considerable time after the sweetened variety.

Latterly, the manufacture of condensed separated milk has been commenced, and a good market for it is found, particularly in England. The chemical composition is somewhat as follows : ${ }^{1}$

\begin{tabular}{|c|c|c|c|c|c|c|}
\hline \multirow{5}{*}{$\begin{array}{l}\text { Water } \\
\text { Fat... } \\
\text { Protein } \\
\text { Sugar } \\
\text { Salts }\end{array}$} & \multicolumn{4}{|c|}{ Unsweetened. } & \multicolumn{2}{|c|}{ Sweetened. } \\
\hline & $\begin{array}{l}\cdots \\
\cdots\end{array}$ & ... & 0.3 & , & 0.2 & , \\
\hline & $\ldots$ & ... & $12 \cdot 4$ & , & $11 \cdot 6$ & , \\
\hline & $\cdots$ & $\cdots$ & $15 \cdot 7$ & , & 59. & ", \\
\hline & $\ldots$ & $\cdots$ & $2 \cdot 6$ & ", & $2 \cdot 2$ & ," \\
\hline & & & 1000 & , & $100^{\circ} 0$ & \\
\hline
\end{tabular}

In the sweetened milk the sugar is one-quarter milk sugar and three-quarters cane sugar.

The largest manufacturers of condensed milk are found in Switzerland, England, Holland, America and Norway.

Dried milk or milk powder is prepared by evaporating the milk to dryness, and it contains all the constituents of milk

1 N. Engström, Handboki Mejerihushaillning. Landtbrukets Bok III, Stock. holm, 1902, p. 621 . 
except the water. Before the manufacture of condensed milk was commenced, an attempt was made in America to make a dry preparation of milk which was pressed into tablets, but the attempt was not successful. Since then many more or less successful attempts have been made, but it is only during the last few years that the manufacture of a satisfactory material has been in operation on a large scale. The difficulties and failures which had previously attended the manufacture of dried milk or milk meal were due either to the fat becoming rancid, or the casein, which is ordinarily present in milk in a collodial state, became changed by the heat, and was then insoluble in water. A good dried milk when stirred with water ought to give a fluid which, in taste and appearance as well as in its chemical and physical properties, exactly resembles milk.

Even now there is no dried milk preparation which exactly fulfils these conditions, but there are several which approach closely to the ideal, and they are used in large quantities both for baking and in the manufacture of such foods as chocolate. Every advance in this branch of the subject is to be welcomed, for success means a solution of that very important problem in modern dairy work, viz., the disposal of the ever-increasing quantity of separated milk. Up to the present, separated milk has been used in far larger quantities for this preparation than whole milk, for the material made from the latter has not found such a ready sale.

The best known preparations of dried milk at the present time are those of Dr. M. Ekenberg (Sweden) and JustHatmaker (America), the manufacture being essentially different in each case.

In the Ekenberg method the milk is evaporated in a drum at $40^{\circ}-45^{\circ} \mathrm{C}$. in vacuum. A nickel cylinder heated internally by steam revolves inside the drum. The milk dries on the surface of the cylinder to a thin layer, which is automatically scraped off and falls into a special receiver, and this dried milk is then ground to powder after being cooled, and further dried in warm air.

In the Just-Hatmaker method, on the other hand, the milk is allowed to flow between two iron cylinders which rotate in opposite directions and are heated by steam to about $130^{\circ}-140^{\circ}$. The milk dries almost instantaneously on the outer 
surfaces of the cylinders to a thin paper-like skin, and is scraped off by knives which are fixed alongside the cylinders. Thus, in this method, a vacuum is not employed. The dried milk, after being scraped from the cylinder, is immediately ground to powder. Although the temperature of the cylinder is $130^{\circ}-140^{\circ}$, the dried milk is not raised to a higher temperature than $100^{\circ}$, for it does not remain sufficiently long in contact with the hot metallic surfaces to allow of all the water being driven off.

In the two processes described above, the product is made from whole milk, and therefore some addition must be made to the milk before drying, in order to give a greater stability to the emulsion which is formed on dissolving the powder in water. For this purpose Hatmaker uses 1-3 per cent. sodium bicarbonate, and Ekenberg 2 per cent. sugar. Such milk powder made from whole milk soon becomes rancid if air has access to it, but when packed in hermetically sealed tins or pressed into tabloids, it is very durable. In the following table are some data regarding the chemical composition of both these varieties of desiccated milk.

\begin{tabular}{|c|c|c|c|c|c|c|c|c|}
\hline & & & & & \multicolumn{2}{|c|}{ Ekenberg. } & \multicolumn{2}{|c|}{ Just-Hatmaker. } \\
\hline & & & & & $\begin{array}{l}\text { Separated } \\
\text { Milk. }\end{array}$ & $\begin{array}{c}\text { Half Whole } \\
\text { Milk. }\end{array}$ & $\begin{array}{l}\text { Separated } \\
\text { Milk. }\end{array}$ & $\begin{array}{l}\text { Half Whole } \\
\text { Milk. }\end{array}$ \\
\hline $\begin{array}{l}\text { Water } \\
\text { Fat ... } \\
\text { Protein } \\
\text { Sugar } \\
\text { Ash... }\end{array}$ & $\begin{array}{l}\ldots \\
\cdots \\
\cdots \\
\cdots \\
\cdots\end{array}$ & $\begin{array}{l}\ldots \\
\cdots \\
\cdots \\
\cdots \\
\cdots\end{array}$ & $\begin{array}{l}\ldots \\
\cdots \\
\cdots \\
\cdots \\
\cdots\end{array}$ & $\begin{array}{c}\ldots \\
\ldots \\
\ldots \\
\ldots \\
\ldots\end{array}$ & $\begin{array}{c}\text { per cent. } \\
5 \cdot 3 \\
1 \cdot 6 \\
28 \cdot 2 \\
57 \cdot 9 \\
7 \cdot 0\end{array}$ & $\begin{array}{c}\text { per cent. } \\
5 \cdot 3 \\
15 \cdot 6 \\
21 \cdot 9 \\
51 \cdot 0 \\
6 \cdot 2\end{array}$ & $\begin{array}{c}\text { per cent. } \\
7 \cdot 0 \\
2 \cdot 0 \\
37 \cdot 0 \\
46 \cdot 0 \\
8 \cdot 0\end{array}$ & $\begin{array}{c}\text { per cent. } \\
4 \cdot 8 \\
28 \cdot 5 \\
24 \cdot 3 \\
36 \cdot 8 \\
5 \cdot 6\end{array}$ \\
\hline & & & & & $100 \cdot 0$ & $100 \cdot 0$ & $100 \cdot 0$ & $100 \cdot 0$ \\
\hline
\end{tabular}

Milk tabloids are made by simply pressing together the dried milk, and naturally, from the practical point of view, this form is the most advantageous.

In addition to the preparations of milk which have been described above, there are plenty of others which are used for special dietetic purposes. Amongst these are the two

1 With 2.5 per cent. fat. 
beverages, Koumiss and Kephir, both of which contain alcohol, and have been known from earliest times. Koumiss is prepared from mare's milk by the nomadic tribes living on the Asiatic Steppes, and kephir from cow's milk by the dwellers in the Caucasus. Kephir is prepared from milk that has previously been boiled, and the so-called kephir grains, the origin of which is not known, are the active agents in causing fermentation.

These grains, which contain the micro-organisms rcquired for the fermentation, have a yellow colour, and measure on an average $0 \cdot 2-5 \mathrm{~cm}$. When dried, as found in commerce, they can be kept for a long time without losing their efficiency, and only require to be first soaked and then washed in luke-warm water before being placed in the milk, which must previously have been boiled and cooled to $20^{\circ} \mathrm{C}$. Small quantities of this milk, after the grains have been strained off, are mixed with four times the quantity of boiled milk cooled down to $20^{\circ}$. The mixture is placed in well-corked champagne bottles, and allowed to ferment at a temperature of $\cdot 12^{\circ}-20^{\circ} \mathrm{C}$. After two or three days the kephir is ready. It is a foaming liquid resembling milk or cream, and possessing a pleasant refreshing acid taste. The milk sugar and the casein of the milk have undergone certain changes under the action of the organisms present in the kephir grains. A portion of the milk sugar has been converted into lactic acid, whilst another portion has fermented to give alcohol and carbon dioxide, the latter imparting a refreshing taste to the liquid. In consequence of the formation of acid, the casein has separated into finelydivided forms, so that it is easily digested by weak or sick persons. This beverage, it is easy to understand, has gained considerable popularity on account of these properties.

The most complete study of the bacteria in kephir grains has been made by Dr. v. Freudenreich ${ }^{1}$ of Bern, and he has isolated the four following micro-organisms. Two streptococci, which form lactic acid from the milk sugar, one of which possesses at the same time the power of inverting part of the milk sugar, which is then acted upon by a yeast-Saccharomyces kefir-and converted into alcohol and carbon dioxide.

1 Landwirt. Jahrbuch der Schweiz, 1896. Centralblatt für Bakt. Section 2, Vol. III, p. 47. 
This yeast differs markedly from the ordinary beer and wine yeasts. The fourth organism is a bacillus, Dispora caucasica (Kern ${ }^{1}$ ) or Bacillus caucasicus, as v. Freudenreich names it. What part is played by this bacillus in the kephir fermentation has not yet been satisfactorily determined. None of the above-mentioned micro-organisms is alone able to bring about the kephir fermentation, but it requires milk to be inoculated with all four at the same time. Thus there is clearly a true symbiosis amongst the four different organisms.

E. J. Nikolajewa, ${ }^{2}$ who has more recently investigated this subject, finds that only two micro-organisms are essential for the specific kephir fermentation, and these are Bacillus caucasicus and the lactose fermenting yeast to which the name Torula kephir is given. This investigator was able to prepare kephir with the help of those two organisms alone. The Bacillus caucasicus forms the stroma of the kephir grains.

Hammarsten ${ }^{3}$ gives the following details as to the composition of kephir:

Kephir from the same milk.

4 days old.

$\begin{array}{lccccc}\text { Water } & \ldots & \ldots & \ldots & 88.64 & \text { per cent. } \\ \text { Fat ... } & \ldots & \ldots & \ldots & 3.63 & , \\ \text { Casein } & \ldots & \ldots & \ldots & 2 \cdot 59 & , \\ \text { Albumin } \ldots & \ldots & \ldots & 0.41 & , \\ \text { Peptone } & . . & \ldots & \ldots & 0.09 & , \\ \text { Sugar } & \ldots & \ldots & \ldots & 2.38 & , \\ \text { Salts } & \ldots & \ldots & \ldots & 0.62 & , \\ \text { Lactic acid } & \ldots & \ldots & 0.83 & , \\ \text { Alcohol } & . . & \ldots & \ldots & 0.81 & ,\end{array}$

6 days old.

$89 \cdot 00$ per cent.

$\begin{array}{ll}3.63 & , \\ 2.56 & , \\ 0.39 & , \\ 0.12 & , \\ 1.67 & , \\ 0.63 & , \\ 0.90 & , \\ 1.10 & , \\ 100.00 & \end{array}$

Koumiss, which, as previously mentioned, is usually prepared from mare's milk, resembles kephir in many respects, but it has not come into such general use. Old or dried koumiss is taken as the inciter of fermentation, which is also due to a symbiosis of several different micro-organisms (a lactic acid bacterium, a yeast, and a bacillus). The microbiology of koumiss fermentation is, however, not yet laid clear. ${ }^{4}$

Fleischmann ${ }^{5}$ gives the following composition to a six to eight days' old koumiss prepared from mare's milk:

${ }^{2}$ Bull. de la Soc. Imp. des Naturalistes de Moskou, 1881, No. 3. Biol. Centralblatt, Vol. II, p. 137.

${ }_{2}$ Bull. du Jardin Imp. Botanique de St. Petersburg, 1907, No. 4.

3 Upsala läkarfören. förhandl, Vol. XXI, 1886, p. 255.

4 Schipin, Centralblatt für Bakt. Section 2, 1900, Vol. VI, p. 775.

5 Lehrbuch der Milchwirtschaft. Fourth Edition. Leipsic, 1907, p. 407. 


\begin{tabular}{|c|c|c|c|c|c|c|c|c|c|}
\hline Water & & & & & & & & 91.535 pe & r cen \\
\hline Fat ... & $\ldots$ & $\ldots$ & $\ldots$ & $\ldots$ & $\ldots$ & $\ldots$ & $\ldots$ & $1 \cdot 274$ & , \\
\hline Protein & $\ldots$ & $\ldots$ & $\ldots$ & $\ldots$ & $\ldots$ & $\ldots$ & $\ldots$ & 1.913 & ,", \\
\hline Sugar & $\ldots$ & $\ldots$ & $\ldots$ & $\ldots$ & $\ldots$ & $\ldots$ & $\ldots$ & $1 \cdot 253$ & , \\
\hline Salts... & & $\ldots$ & $\ldots$ & $\ldots$ & $\cdots$ & $\cdots$ & $\ldots$ & 0.293 & ", \\
\hline Carbon & iox & de, & ree & and & consh & ined & .• & 0.876 & 3 \\
\hline $\begin{array}{l}\text { Alcohol } \\
\text { Lactic ac }\end{array}$ & & ... & $\ldots$ & $\ddot{. .}$ & $\begin{array}{l}\cdots \\
\ldots\end{array}$ & $\ldots$ & $\begin{array}{l}\cdots \\
\ldots\end{array}$ & $\begin{array}{l}1.850 \\
1.006\end{array}$ & ,", \\
\hline & & & & & & & & 00.000 & \\
\hline
\end{tabular}

Specific gravity at $15^{\circ} \mathrm{C} 1 \cdot 008-1 \cdot 020$.

"Leben," "Mazoun," and "Yoghurt," are other sour beverages prepared from milk, the first in Egypt, the second in Armenia, and the last in Bulgaria. Yoghurt has come into. prominence in the last few years, because of the Bacillus bulgaricus which it contains. This organism is a very powerful exciter of lactic acid fermentation, and, according to E. Metschnikoff, it acts as a sort of disinfectant in the large intestine, and thereby checks the chronic toxic poisoning caused by the putrefaction organisms. Metschnikoff, who is of the opinion that the putrefactive fermentation which usually takes place -in the large intestine tends to shorten life and that if these putrefactive changes could be checked human life would be prolonged, recommends the use of milk containing the Bacillus bulgaricus. The unusually long life enjoyed by many of the Bulgarian country population would thus be explained, for they are known to consume large quantities of yoghurt daily. Cultures of the micro-organisms present in yoghurt can now be bought all over the world under the name of Lactobacillin, Bulgarian Maya, \&c. By allowing milk to become partially sour, and then adding some of the culture, yoghurt can be obtained in a few hours if the temperature is kept at about $40^{\circ}$.

Fuhrmann ${ }^{1}$ has prepared yoghurt in this way, and after seven hours at $40^{\circ}$ it had the following composition:

\begin{tabular}{|c|c|c|c|c|c|c|c|}
\hline \multirow{3}{*}{$\begin{array}{l}\text { Protein } \\
\text { Fat ... } \\
\text { Milk sugar }\end{array}$} & \multirow[b]{2}{*}{$\ldots$} & \multicolumn{4}{|c|}{ The milk used. } & \multicolumn{2}{|c|}{ The Yoghurt. } \\
\hline & & $\cdots$ & $\ldots$ & $5 \cdot 780$ & ," & $5 \cdot 740$ & , \\
\hline & $\ldots \quad .$. & $\ldots$ & & $7 \cdot 210$ & & 6.873 & ," \\
\hline Acids, calcu & ulated to & lactic & acid & 0.145 & " & 0.344 & ,", \\
\hline Fat, free dr & ry matter & ... & . $\ldots$. & $14 \cdot 800$ & ," & $14 \cdot 892$ & , \\
\hline & $\ldots \quad \ldots$ & $\ldots$ & .. & - & & 0.090 & ", \\
\hline ldehyde & $\ldots \quad \ldots$ & $\ldots$ & .. $\quad \ldots$ & - & & traces & \\
\hline
\end{tabular}

1 Zeitschr. für Untersuch. der Nahr.-u. Genussmittel, 1907, Vol. XIII, p. 598. 
Since about 1895 the manufacture of certain preparations from the casein of milk has been commenced, and at the present time large quantities are prepared both as additions to foods and also for special use in cases of disease. For both these purposes casein offers great advantages, for it is easily absorbed by the organism without forming evil-smelling decomposition products, as is the case with most other protein substances. Further, protein can be bought more cheaply in the form of casein than, for example, in eggs.

In all the preparations which are to be found at the present time in commerce, and have been prepared for the abovementioned purposes, the insoluble casein has been converted into a soluble alkali compound. ${ }^{1}$

For the sake of comparison the chief properties of the most important food preparations of casein will be found in tabular form on the opposite page.

Casein is now prepared in considerable quantities for purely technical purposes by precipitating separated milk with commercial hydrochloric or sulphuric acid, and then drying and grinding the precipitated mass. In certain districts, e.g. the Argentine, strongly acid whey is used for the precipitation. When prepared in this way the casein is naturally insoluble in water, and it finds considerable application in certain branches of industry. In some cases advantage is taken of the property which casein possesses of giving glue-like substances with alkalies. Casein, after treatment with formaldehyde, yields a substance which resembles in its properties gelatine or celluloid. The first of these substances is used in considerable quantity in dyeing and calico-printing, and the second for the preparation of photographic films and for imitation tortoiseshell, ivory, amber, horn, etc.

The whey which remains after the precipitation of the casein of milk by rennet serves for the preparation of milk sugar. This sugar is almost exclusively used as an addition to mixtures of cow's milk and water (cream being sometimes added) in the feeding of infants. The addition of milk sugar in such cases makes up the percentage of that substance in the food, for it

1 A detailed account of a large number of such preparations are to be found in Max Heim's Die künstlichen Nührpräparate und Anregungsmittel, Berlin, 1901, pp. 68-94; also in Die Magermilch-Verwertung in den Molkereren, by C. Knoch, Leipzig, 1903, pp. 120-134. 


\begin{tabular}{|c|c|c|c|c|c|c|c|c|}
\hline \multirow{5}{*}{ 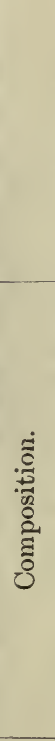 } & 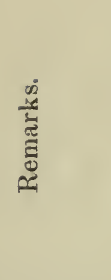 & & 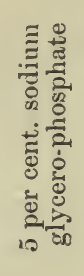 & & & & & 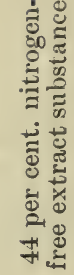 \\
\hline & 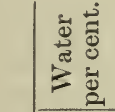 & $\cong$ & $\dot{\infty}$ & $\dot{\dot{\theta}}$ & $\stackrel{\leftrightarrow}{\bullet}$ & $\begin{array}{l}\dot{\varphi} \\
\stackrel{\text { il }}{\sim}\end{array}$ & $\hat{\dot{\infty}}$ & $\stackrel{9}{\dot{\theta}}$ \\
\hline & 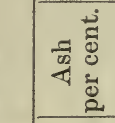 & $\stackrel{\leftrightarrow 0}{\circ}$ & $\stackrel{10}{0}$ & $\stackrel{5}{i s}$ & $\ddot{r}$ & + & i & $\stackrel{\leftrightarrow}{\ddot{q}}$ \\
\hline & 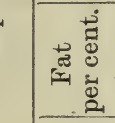 & $\dddot{\square}$ & 1 & 1 & 1 & 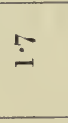 & $\ddot{\theta}$ & $\stackrel{\leftrightarrow}{\square}$ \\
\hline & 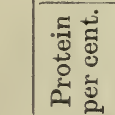 & $\dot{\infty}$ & $\dot{8}$ & $\stackrel{P}{\dot{D}}$ & $\begin{array}{l}\infty \\
\dot{9} \\
\dot{\infty}\end{array}$ & $\stackrel{10}{+1}$ & $\overrightarrow{\dot{\infty}}$ & $\stackrel{\leftrightarrow}{\stackrel{\rho}{\rho}}$ \\
\hline \multicolumn{2}{|r|}{ 晜 } & 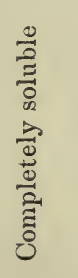 & 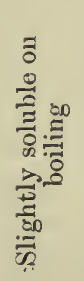 & 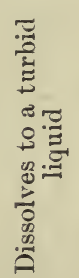 & 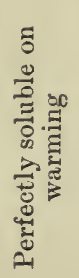 & $\begin{array}{c}\stackrel{0}{3} \\
\stackrel{8}{3} \\
\vdots 2\end{array}$ & $\begin{array}{l}\frac{0}{3} \\
\frac{1}{0} \\
\infty\end{array}$ & 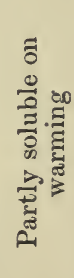 \\
\hline \multicolumn{2}{|r|}{ 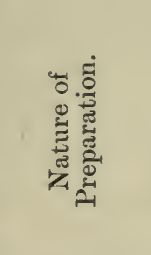 } & 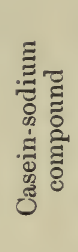 & 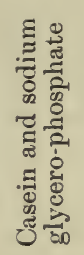 & 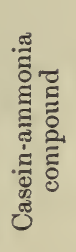 & 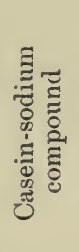 & 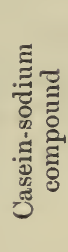 & 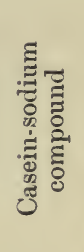 & 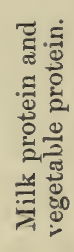 \\
\hline \multirow{2}{*}{\multicolumn{2}{|c|}{ 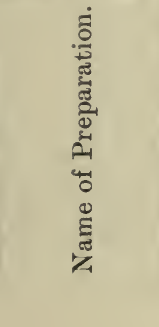 }} & $\begin{array}{c}\stackrel{5}{\circ} \\
\stackrel{0}{\circ} \\
\text { ค1. }\end{array}$ & 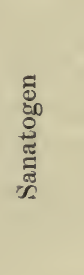 & 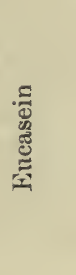 & 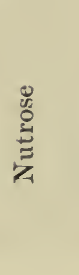 & 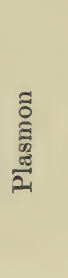 & 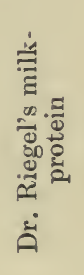 & 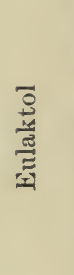 \\
\hline & & & & & & & & $\mathbf{P}$ \\
\hline
\end{tabular}


has naturally diminished owing to the dilution. The whey, which is used for the preparation of milk sugar, is generally evaporated, after the precipitation of any nitrogenous substances which may be present, in a vacuum pan, and the crude milk sugar allowed to crystallise out. It is then purified according to various methods, decolorised, refined, etc., so that the final product is quite colourless.

\section{Examination of Preserved Milik and other Milk Preparations.}

\section{I.-Sterilised and Pasteurised Milk.}

The chemical analysis of sterilised and pasteurised milk is naturally performed in exactly the same manner as in the case of milk alone. Preparations of this sort ought not to have a brownish-yellow colour, nor ought the fat globules have run together so that the drops are visible to the naked eye. Neither ought it to be dificult to get the layer of fat which forms in the bottles to mix again with the milk. When homogenised milk is being dealt with the fat cannot be estimated by any of the extraction methods, owing to the reason given on page 51, for these all give results which are too low, sometimes as much as 0.5 per cent. The Röse-Gottlieb method is, however, quite satisfactory, for the fine state of division of the fat particles in the emulsified milk has no effect upon the result. Gerber's method also gives good results if the samples are whirled for a considerably longer time than usual. Should the centrifuge not be one that can be heated, then the samples must be warmed and whirled several times until no more fat separites. The degree of acidity of pasteurised milk is often somewhat lower than that of the unheated milk, owing to a loss of free carbon dioxide during heating. Storch's test (p. 98) will show whether the milk has been heated to more than $80^{\circ} \mathrm{C}$., but there is no means of learning definitely how far the heating has exceeded this temperature. By means of the so-called Schardinger reaction ${ }^{1}$ it is possible to get a good idea of the temperature to which the milk has been heated, and also the length of time. Schardinger found that when a certain

1 See Buttenberg, Zeitschrift f. Unt. der Nahr.- u. Genussmittel, 1906, Vol. II, p. 377. 
quantity of the following solution (Schardinger's reagent)5 c.c. of saturated alcoholic solution of methylene blue +5 c.c. of formalin +190 c.c. of distilled water-was added to ordinary normal milk it was decolourised in about ten minutes. The test is carried out by mixing 20 c.c. of milk with 1 c.c. of Schardinger's reagent, placing the mixture in a water-bath at $45-50^{\circ}$, and noting the time which elapses before the colour entirely disappears.

This test depends upon the presence of a specific enzymethe aldehydereductase-in the milk. The enzyme is destroyed more or less quickly at temperatures above $70^{\circ}$, so that if the milk has been heated to $80^{\circ}$ the colour is not discharged at all. If, on the other hand, the colour disappears after about 30 minutes, the milk was either heated for a few minutes at $75^{\circ}$ or pasteurised for 15 minutes at $70^{\circ}$. Further information relative to the degree of pasteurisation can be obtained if the test proposed by Bonnema ${ }^{1}$ is used. This is carried out by placing the milk which is to be investigated in flasks provided with rubber stoppers. The flasks, which hold 100 g. milk, are sterilised and then filled to the stopper, after which they are placed in an incubator at $37^{\circ}$, or failing this a water-bath can be used. The nature of the fermentation which goes on in the flasks admits of an estimate of the degree of pasteurisation being formed. It is possible to distinguish between the following main types of fermentation:

1. Lactic acid fermentation.-Homogeneous and solid coagulum, with few bubbles of gas and little serum, indicates that the milk was unheated, or pasteurised at a low temperature.

2. Butyric acid fermentation.-The coagulum swims as a cake, permeated with gas bubbles, on the upper part of the liquid. The serum below is clear. There is a lively evolution of gas and the smell of butyric acid. The stoppers often blow out. These signs denote a milk pasteurised between $75^{\circ}$ and $90^{\circ}$.

3. An intermediate form between 1 and 2 indicates that the milk was pasteurised for 15 to 20 minutes at $70^{\circ}$.

4. Peptone fermentation.-At first the milk is evenly coagulated and in fine particles. Afterwards a clear zone forms below the layer of cream and spreads gradually downwards. In this case the milk was pasteurised for 10 minutes at $95^{\circ}$ or above.

${ }^{1}$ Chemiker-Zeitung, 1905, V́ol. XXIX, p. 182. 
As the anaerobic butyric acid bacteria are killed sooner than the whey bacteria (peptonising bacteria) it is possible, as has been shown above, to gain some idea as to the temperature to which the pasteurisation has been carried by the changes which take place when the milk is kept in the incubator.

Perfectly sterile milk must naturally remain unchanged when kept in the incubator at $35^{\circ}$ to $40^{\circ} \mathrm{C}$.

\section{II.-Buddised MilK.}

In the examination of milk which has been treated with hydrogen peroxide according to Budde's or any other method, the two main points to be investigated are, firstly, whether undecomposed hydrogen peroxide is present in the milk and in what quantity, and, secondly, whether the milk is sterile or not.

The milk should first be tasted, and if an excess of only $0.05 \mathrm{~g}$. hydrogen peroxide per litre is present a characteristic taste may be detected. ${ }^{1}$ When the quantity is less than this the peculiar flavour is no longer noticed. As regards the qualitative and quantitative detection of hydrogen peroxide in milk, full details are given on p. 122.

To prove the sterility of the milk it must be placed in a closed flask in the incubator at a temperature of $35^{\circ}$ to $40^{\circ}$, just as was done with milk sterilised by heat. If the milk is sterile it should undergo no change, but if not, and the hydrogen peroxide has only been used to make it more durable, then it and a sample of normal milk should be kept for a few days under the same conditions and tested for the degree of acidity every few hours.

Sometimes it happens that the acidity of the milk heated with hydrogen peroxide only increases slightly, but an objectionable smell and unpleasant taste are to be noticed. This is caused by the lactic acid bacteria having been killed, and the remaining putrefactive bacteria have gained the upper hand. Such milk must naturally be rejected absolutely. It is essential that the milk treated with hydrogen peroxide should either be sterile or have been treated so little that the lactic acid bacteria still predominates, and souring can take place in the normal manner.

${ }^{1}$ Chr. Barthel, lievue Gén. du Lait, Vol. II, p. 289. 


\section{III.-CONdENSED MiLK.}

The best way to get a good average sample of condensed milk is to pass a portion of the contents of a tin through a sieve, and then, after stirring, the sample for analysis may be drawn. Condensed milk is examined in exactly the same way as ordinary milk, after weighing out the quantity required and diluting it with water as advised in the directions for use. If no directions are given the milk should be so diluted that the specific gravity is slightly higher than that of ordinary milk, or about 1.032. The percentage of fat is best determined by the Röse-Gottlieb method (p. 52). The amount of sugar is found by deducting the sum of the fat, protein, and salt from the dry matter. If a quantitative determination of the amounts of milk and cane sugar is wanted, the former is first made by boiling with Fehling's solution after first precipitating the protein substances (p. 85). Another portion is inverted with hydrochloric acid, and the invert sugar determined by Fehling's solution. The cane sugar can then be calculated from the following formula:-

$$
\begin{array}{r}
R=\left(\mathrm{I}-M \frac{100}{134}\right) 0.95 \\
\mathrm{I}=\text { Invert sugar } \\
M=\text { Milk sugar. }
\end{array}
$$

100 parts of invert sugar and 134 parts milk sugar give the same quantity of $\mathrm{Cu}_{2} \mathrm{O}$.

In controlling the manufacture of condensed milk there are several approximate methods which are used for determining the amount of cane sugar. The two most important are :-

1. The total polarisation is first ascertained, and then the amount of milk sugar is determined by multiplying the percentage of ash by 6.5 . The difference between the total sugar and the milk sugar gives of course the cane sugar.

2. The fat-free dry matter is first determined $(a)$ from the specific gravity and the percentage of fat, and then $(b)$ from the percentage of ash multiplied by 12 . The quantity of cane sugar is then obtained by subtracting $b$ from $a$.

A qualitative test for cane sugar in milk or in milk sugar is 
that of Seliwanoff as modified by Carlsson, ${ }^{1}$ which is carried out as follows :- 10 c.c. of milk are taken, or $0.5 \mathrm{~g}$. of milk sugar dissolved in 10 c.c. of water, and warmed in a test-tube with $50 \mathrm{mg}$. of resorcin and 0.5 c.c. of hydrochloric acid $(25$ per cent.). In the presence of cane sugar a red coloration is obtained after boiling for a few minutes. With less than 1 per cent. of cane sugar the reaction is somewhat indistinct, but for more than 1 per cent. it is very reliable. Still more sensitive is the test employed by Rothenfusser for detecting the saccharose of calcium saccharate when the latter has been mixed with milk or cream (p. 117).

The condensed milk must also be examined for preservatives other than cane sugar.

\section{IV.-Desiccated Milk or Milk Powder.}

The majority of samples of desiccated milk prepared up to the present do not give by any means a perfectly homogeneous milk-like emulsion when mixed with the prescribed quantity of water. On account of the difficulty of getting a satisfactory average sample from such an artificial mixture it is best to analyse the dry substance.

The water is determined by drying a few grams of the powder in a drying bottle at $100^{\circ}$ to $105^{\circ} \mathrm{C}$., and the fat either by the Soxhlet or Röse-Gottlieb method. According to H. Haupt ${ }^{2}$ this is best done by taking $1 \mathrm{~g}$. of air-dry powder and shaking it vigorously in a Gottlieb tube for three to five minutes with 9 c.c. of warm water. Then 2 c.c. of 20 per cent. ammonia are added and the shaking repeated until complete solution has been effected. After one or two minutes 10 c.c. of 96 per cent. alcohol are added, the mixture again shaken, and the determination completed in the ordinary way.

The protein is generally determined by Kjeldahl's method, and the nitrogen multiplied by 6:37. To determine the milk sugar the dried milk must first be dissolved in water, the quantities which are directed being taken, and when by warming and stirring a satisfactory solution is obtained it is treated like ordinary milk. Some milk powders contain cane sugar, and to

I Pharm. Centralblatt, 1903, p. 133.

2. Zeitschr. für Unt. d. Nahr.- u. Genussmittel, 1906, Vol. XII. p. 217. 
determine the quantity of this sugar along with the milk sugar the method employed for condensed milk (p. 213) can be used.

If the dried milk contains carbonate or bicarbonate of soda, either of these may be easily detected by the alkaline reaction which the powder moistened with water shows to litmus paper.

Adulteration of desiccated milk with foreign substances is not likely, but it is advisable to subject it to a microscopic examination in addition to the chemical. When starch has been added it is easily detected by the blue coloration with iodine.

\section{V.-CASEIN.}

The dry powdered casein which is used for various technical purposes varies in purity according to the method of manufacture. Sometimes sand is found along with the casein, or if insufficiently washed some of the acid used for the precipitation of the casein may be present. Small quantities of milk sugar, etc., are also sometimes present. A marked yellow colour points to the presence of large quantities of lime salts. The amount of sand is easily determined by ignition, treatment with dilute hydrochloric acid, filtration, burning of the filter and weighing of the sand. The presence of hydrochloric or sulphuric acid is detected as usual by an addition of $\mathrm{AgNO}_{3}$ or $\mathrm{BaCl}_{2}$ in the ordinary way. Milk sugar is estimated by Fehling's solution, and the percentage of casein by determining the nitrogen by Kjeldahl's method and multi. plying the amount found by $6 \cdot 38$. 


\section{PART V-DECOMPOSITION PRODUCTS OF MILK, BUTTER, AND CHEESE}

\section{Decomposition Products of Milk.}

MILK in the cow's udder is not sterile under ordinary conditions, for the milk ducts, even the finest, always harbour a greater or less number of bacteria. The number of bacteria present in the udder is only small; generally white and yellow micrococci are the chief ones to be found, and these have no effect upon the milk. ${ }^{1}$ Whether these bacteria get into the udder through the teat or from the blood is not yet proved, but there are many reasons to support the first view. Under normal conditions the number of bacteria in the udder is relatively small, which is due to the bactericidal power of the tissues of the udder. From the moment that the milk leaves the udder it becomes infected with all sorts of micro-organisms from the air, food, litter, excrement of the animals, etc.

The majority of the micro-organisms in milk never develop further, for they are overpowered by others which find there conditions more favourable to their development. To the latter varieties belong the lactic acid bacteria, as well as the Coli- and Aerogenes bacteria. After some time the lactic acid bacteria (if the milk has not been well cooled) have so increased that the other species of bacilli cease to develop, and after 36 to 48 hours at ordinary temperatures 95 to 100 per cent. of the bacterial flora is composed of these lactic acid bacteria. This happens of course under normal conditions. At higher temperatures, e.g. $37^{\circ} \mathrm{C}$., the relation is somewhat different, for then the Coli- and Aerogenes bacteria develop most vigorously, and may even overshadow the lactic acid bacteria. When the degree of acidity of the milk, due to the conversion of the milk sugar into lactic acid, has reached about 0.8 per cent., the lactic

${ }^{1}$ Chr. Barthel, "Die Mikroorganismen in der Stallluft, in der frisch gemolkenen Milch und im Euter der Kuh," Milch-Zeitung, 1903, p. 626. Reprinted in pamphlet form by M. Heinsius Nachfolger, Leipzig. 
acid bacteria cease to develop, but they begin again if the lactic acid which they make is neutralised, e.g. by the addition of chalk. If milk is left undisturbed for some time and plenty of air can have access, then decomposition by moulds, such as the ordinary milk mould, Ö̈dium lactis, as well as Penicillium, Aspergillus, Mucor, etc., continues. The moulds oxidise, that is burn up, the acids that have been formed, and prepare the way for proteoclastic bacteria, which generally prefer a neutral or faintly alkaline nutritive medium. It may further be mentioned that the moulds themselves attack the casein of milk. The proteoclastic bacteria, chief amongst which are the hay bacteria (B. subtilis), decompose casein with the formation of albumoses and peptones. Afterwards the decomposition mainly follows the lines already sketched for the ripening of cheese (p. 182). In addition to the aerobic hay bacilli are putrefactive bacteria, both acrobic and anaerobic, and these actively destroy the protein. Amongst these bacteria are Bacillus faecalis alcaligcnes (Petruschky), Bacillus putrificus (Bienstock), etc.

The splitting-up of the milk fat into glycerine and free fatty acid is accomplished much more slowly than in the case of the milk sugar or the protein. Bacillus fuorescens liquefaciens is the most active of this group.

It takes a very long time, at least several months, for milk to completely decompose. The slowness of the operation is chiefly caused by the large amount of acid which is formed in the early stages, for this naturally has a strong inhibitory influence upon bacterial life.

In pasteurised milk the spontaneous decomposition takes another direction owing to the lactic acid bacilli having been destroyed. If the milk is not infected afresh by lactic acid bacteria none of this acid can be produced, and the first decomposition-changes of normal unheated milk do not occur. Instead of that the milk undergoes putrefaction through the spore-forming hay and putrefaction bacteria that have survived the pasteurisation.

When pasteurised milk is kept at a higher temperature, say about $40^{\circ} \mathrm{C}$., under anaerobic conditions a vigorous butyric acid fermentation sets in which causes the glass bottle (if the milk is in one) to burst. The inciters of the fermentation are chiefly the anaerobic bacteria: Granulobacillus saccharobutyricus im- 
mobilis liquefaciens and Granulobacillus saccharobutyricus mobilis non liquefaciens, which have been studied by Schattenfroh and Grassberger.

\section{Lactic Acid.}

Through the agency of the lactic acid bacteria (Bacterium lactis acidi, Leichmann $)^{1}$ which are found almost everywhere the milk sugar is converted into lactic acid, without formation of secondary products, according to the equation

$$
\mathrm{C}_{12} \mathrm{H}_{22} \mathrm{O}_{11}+\mathrm{H}_{2} \mathrm{O}=4 \mathrm{C}_{3} \mathrm{H}_{6} \mathrm{O}_{3} \text {. }
$$

This acid is the dextrorotary modification of lactic acid, $\boldsymbol{a}$-oxypropionic acid, and has the constitutional formula

\section{$\mathrm{CH}_{3} . \mathrm{CH}(\mathrm{OH}) \mathrm{COOH}$.}

When milk sours spontaneously a mixture of inactive and dextrorotary lactic acid is obtained, because lactic acid bacteria other than those mentioned above take part in the decomposition. Certain of these bacteria give rise to the lævorotary modification of lactic acid.

Lactic acid is estimated quantitatively by titration with standard alkali in the manner described for the determination of the degree of acidity of milk (p. 31). The method is not absolutely correct, but it is the one employed in the majority of cases. $\Lambda$ fter titration, the acidity of normal milk must naturally be deducted. If a qualitative test only is needed for lactic acid, then Uffelmann's reagent can be used with advantage. It consists of a 1 per cent. carbolic acid solution, to which 10-15 drops of ferric chloride have been added to each 100 c.c. In applying the test, a few drops of milk are added to 10 c.c. of the reagent, and in the presence of lactic acid the violet-blue colour changes to a greenish-yellow. This test was originally worked out for the detection of lactic acid in the contents of the stomach.

To determine what modification of lactic acid is formed, the zinc salt is generally prepared, and the following method gives satisfactory results :

One litre of well-mixed sour milk is filtered through a folded filter, which is then repeatedly washed with cold water. The filtrate is evaporated to dryness on the water-bath, the residue

$1 \mathrm{Chr}$. Barthel, "Contribution à la connaissance de la répartition des ferments lactiques en dehors du lait," Revue Gén. du Lait, Vol. V, p. 223. 
made strongly acid with phosphoric acid and shaken out with ether in a separating funnel. After the ether has been distilled off, a yellow, syrupy residue is left, which is dissolved in water and filtered. The filtrate is now boiled with zinc carbonate, again filtered, and the filtrate evaporated, at first on the waterbath and afterwards in vacuum over sulphuric acid. The zinc lactate which crystallises out is dried between filter-paper, dissolved in a small quantity of water, and allowed to crystallise over sulphuric acid. The recrystallised zinc salt, which is usually sufficiently pure for the following examination, is dried again between filter-paper and then over concentrated sulphuric acid to free it from the last traces of moisture. The thoroughly air-dried zinc lactate is analysed, and the pereentage of water of crystallisation and of zinc determined, as well as its behaviour towards polarised light.

Air-dry inactive zinc lactate loses three molecules of water of crystallisation $=18.18$ per cent. when heated to $110^{\circ}$, whilst air-dry active zine lactate loses only two molecules $=12.9$ per cent.

The amount of zinc oxide in anhydrous zinc lactate is 33.33 per cent. For the polarimetric examination, the solution of the zinc salt must be as free as possible from colour, because the rotation is not very powerful.

\section{Volatile Fatty Acids.}

When milk decomposes, there is always found a greater or less quantity of fatty acids, due to the action of different micro-organisms. In the lactic acid fermentation, in so far as this is carried on by bacteria other than Bacterium lactis acidi, small quantities of acetic and formic acids are also formed. Butyric acid can arise both directly from milk sugar by the action of the specific butyric acid bacteria mentioned in the introduction to this chapter, and, secondarily, from calcium lactate, as shown by Pasteur. Fitz has also proved that caproic, valeric, and, under certain conditions, propionic and acetic acids are formed. Finally, volatile fatty acids can also arise from the action of certain micro-organisms upon the easein. 


\section{(a) Qualitative Estimation.}

The best way to detect and separate the different volatile fatty acids is to form the silver salts as in the method worked out by Orla Jensen. ${ }^{1}$ These silver salts can easily be prepared from solutions of the free acids and silver carbonate, or by precipitation of the alkali salts with silver nitrate. They do sot contain water of crystallisation, are easily dried in vacuum over sulphuric acid, and by careful ignition the silver is quickly obtained in a pure state. In the following table are given the solubilities of the silver salts, and the percentages of silver as found by Orla Jensen :

\begin{tabular}{|c|c|c|c|c|c|}
\hline & & & & $\begin{array}{l}100 \text { parts of water } \\
\text { dissolve at } 20^{\circ} \mathrm{C} \text {. }\end{array}$ & Per cent. Ag. \\
\hline $\begin{array}{l}\text { Silver acetate } \\
\text { Silver propionate } \\
\text { Silver butyrate ... } \\
\text { Silver valeriate ... } \\
\text { Silver capronate... } \\
\text { Silver caprylate ... }\end{array}$ & $\begin{array}{l}\cdots \\
\cdots \\
\cdots \\
\cdots \\
\cdots \\
\cdots\end{array}$ & $\begin{array}{l}\cdots \\
\cdots \\
\cdots \\
\cdots \\
\cdots\end{array}$ & \begin{tabular}{l|}
$\cdots$ \\
$\cdots$ \\
$\cdots$ \\
$\cdots$ \\
$\cdots$ \\
$\cdots$
\end{tabular} & $\begin{array}{l}1.037 \text { parts } \\
0.836 \quad, \\
0.489 \quad, \\
0.185 \quad, \\
0.089 \quad, \\
0.018 \quad,\end{array}$ & $\begin{array}{l}64 \cdot 67 \\
59 \cdot 67 \\
55 \cdot 38 \\
51 \cdot 67 \\
48 \cdot 43 \\
43 \cdot 03\end{array}$ \\
\hline
\end{tabular}

It will be seen that the solubility of the silver salts decreases rapidly with the rise of molecular weight, and therefore fractional crystallisation of the salts is an excellent way of separating the volatile fatty acid.

If the procedure now to be described is followed, very satisfactory results are obtained. When the milk culture cannot easily be filtered through paper, the casein is precipitated with a little dilute sulphuric acid, and the filtration is then performed without difficulty. Sufficient sulphuric acid is added to the filtrate to liberate the volatile acids, and they are then distilled off with steam, the volume of liquid in the flask being kept constant, so that from 200 c.c. of the filtrate 1 litre of distillate may be got. Ordinary tap water, which has been boiled with sulphuric acid to drive off all the carbon dioxide, is used as the generator of steam. The distillate is titrated with baryta

1 "Biologische Studien über den Käsereifungsprogress unter spezieller Beriicksichtigung der flïchtigen Fettsaüren," Landwirtschaftliches Jahrbuch der Schweiz, 1904, p. 319. 
water, concentrated, filtered in order to get rid of any traces of barium carbonate, and neutralised with nitric acid. As only $9 \cdot 2 \mathrm{~g}$. of barium nitrate are soluble in 100 parts of water at $20^{\circ} \mathrm{C}$., the solution of the barium salt must not be concentrated more than to leave 15 c.c. of water for each 100 c.c. $N / 10$ baryta used.

The fractional precipitation of the silver salts now begins by adding a few cubic centimetres of normal silver nitrate to the solution of the barium salt. The precipitate is filtered off, a little more silver nitrate added, and, proceeding in this way, 6 to 8 fractions are obtained. Each precipitate is dried between filter paper, and placed in vacuum over sulphuric acid. Afterwards the amount of silver is determined in each of these precipitates by carefully igniting it; the percentage of silver is compared with Jensen's table for the identification of the different acids.

\section{(b) Quantitative Estimation.}

Jensen's method for the qualitative detection of the various volatile fatty acids, as described above, cannot be used for the quantitative analysis of these acids. The filter and the salts which are on it soak up so much of those salts which are still in solution that considerable loss occurs, particularly of the lower acids, which are the last to be precipitated. Owing to the solubility of the silver salts of these acids, it is impossible to wash them. Duclaux ${ }^{1}$ has, however, worked out a method which can be used with satisfactory results. It is based upon the well-known fact that the volatile fatty acids in weak aqueous solution distil more quickly the higher their molecular weight.

Suppose any volatile acid to be in a solution of 1 or at most 2 per cent., then a certain volume, say 110 c.c., is taken and distilled in the ordinary way with a Liebig's condenser. The distillate is collected in 10 fractions in small measuring flasks holding 10 c.c. Each of the fractions is then neutralised with a standard solution of alkali from a burette, and the amount required for each portion is noted.

The three following facts then come into consideration:

1 Traité de Microbioloyie, Vol. III., p. 384, and Vol. IV., p. 685. 
(1) The curve which gives expression to the figures obtained in the distillation is characteristic of the acid in question.

(2) A certain definite relation exists between the quantity of acid which was present in the flask from the commencement onwards, and the quantity which distils over at any given time. It is thus possible, knowing the amount of acid in the first $10,20,30,40$, etc.; c.c. to calculate the original quantity of acid in the distillation flask.

(3) When a mixture of two acids is distilled, each acid behaves as though it alone were present.

Duclaux used lime-water for the titration, for when saturated it has exactly the right alkalinity for titrating the small quantities of acid which occur. The strength of the lime-water is first determined by titrating it against an acid of known strength; phenolphthalein being used as the indicator. If the relation between the amounts of lime-water necessary to neutralise the first $10,20,30,40$, etc., c.c., and the quantity necessary to neutralise the total original amount of acid is found, then there is obtained for each acid a series of numbers, which may, if wished, be expressed by a curve. This curve is characteristic of each separate acid. The figures obtained for the ordinary volatile fatty acids are as follows:

TABLE A.

\begin{tabular}{r|c|c|c|c|c}
\hline & Formic acid. & Acetic acid. & $\begin{array}{c}\text { Propionic } \\
\text { acid. }\end{array}$ & $\begin{array}{c}\text { Butyric } \\
\text { acid. }\end{array}$ & Valeric acid \\
\cline { 2 - 5 } 10 c.c.... & $3 \cdot 5$ & $5 \cdot 9$ & $11 \cdot 5$ & $17 \cdot 3$ & $30 \cdot 5$ \\
20 c.c... & $7 \cdot 2$ & $12 \cdot 2$ & $22 \cdot 8$ & $32 \cdot 7$ & $53 \cdot 0$ \\
30 c.c... & $11 \cdot 3$ & $18 \cdot 7$ & $33 \cdot 5$ & $47 \cdot 0$ & $69 \cdot 5$ \\
40 c.c... & $15 \cdot 5$ & $25 \cdot 6$ & $44 \cdot 0$ & $58 \cdot 5$ & $81 \cdot 0$ \\
50 c.c... & $20 \cdot 2$ & $32 \cdot 7$ & $54 \cdot 0$ & 68.8 & $88 \cdot 5$ \\
60 c.c... & $25 \cdot 5$ & $40 \cdot 4$ & $63 \cdot 3$ & $77 \cdot 5$ & $93 \cdot 5$ \\
70 c.c... & $31 \cdot 1$ & $48 \cdot 7$ & $7 \cdot 5 \cdot 5$ & $84 \cdot 3$ & $96 \cdot 5$ \\
80 c.c... & $38 \cdot 5$ & $57 \cdot 5$ & $81 \cdot 0$ & $90 \cdot 5$ & $98 \cdot 3$ \\
90 c.c... & $48 \cdot 0$ & $67 \cdot 5$ & $88 \cdot 5$ & $94 \cdot 6$ & $99 \cdot 5$ \\
100 c.c... & $59 \cdot 0$ & $80 \cdot 0$ & $95 \cdot 0$ & $97 \cdot 5$ & $100 \cdot 0$ \\
\hline
\end{tabular}

Instead of determining the relation between the quantity of lime-water required for the whole of the acid and that used for the successive fractions, it is simpler to calculate the relation between the lime-water neutralised by the 100 c.c. of distil- 
late and that required for the different fractions. If, for example, the total amount of lime-ivater $=\alpha$, and if for the $3 \mathrm{rd}$ fraction the number $b$ had been read off on the burette, then :

$$
a: b=100: x ; x=\frac{100}{a} . b .
$$

The series of numbers obtained in this way are as follows:-

Table B.

\begin{tabular}{|c|c|c|c|c|c|}
\hline & Formic acid. & Acetic acid. & $\begin{array}{l}\text { Propionic } \\
\text { acid. }\end{array}$ & $\begin{array}{l}\text { Butyric } \\
\text { acid. }\end{array}$ & Valeric acid \\
\hline 10 c.c. .. & $5 \cdot 9$ & $7 \cdot 4$ & $12 \cdot 1$ & $17 \cdot 6$ & $30 \cdot 5$ \\
\hline 20 c.c.... & $12 \cdot 2$ & $15 \cdot 2$ & $24 \cdot 0$ & $33 \cdot 6$ & $53 \cdot 0$ \\
\hline 30 c.c.... & $19 \cdot 0$ & $23 \cdot 4$ & $35 \cdot 3$ & $47 \cdot 5$ & $69 \cdot 5$ \\
\hline 40 c. c.... & $26 \cdot 4$ & $32 \cdot 0$ & $46 \cdot 2$ & $60 \cdot 0$ & $81 \cdot 0$ \\
\hline 50 с.с.... & $34 \cdot 4$ & $40 \cdot 9$ & $56 \cdot 8$ & $70 \cdot 6$ & $88 \cdot 5$ \\
\hline 60 с.с. .. & $43 \cdot 2$ & $50 \cdot 5$ & $66 \cdot 7$ & $79 \cdot 5$ & $93 \cdot 5$ \\
\hline 70 с.с.... & $52 \cdot 8$ & $60 \cdot 9$ & $76 \cdot 2$ & $86 \cdot 5$ & $96 \cdot 5$ \\
\hline 80 c.c.... & $64 \cdot 6$ & $71 \cdot 9$ & $85 \cdot 0$ & $92 \cdot 5$ & $98 \cdot 3$ \\
\hline 90 c.c.... & $79 \cdot 6$ & $84 \cdot 4$ & $93 \cdot 0$ & $97 \cdot 0$ & $99 \cdot 5$ \\
\hline 100 c.c.... & $100 \cdot 0$ & $100 \cdot 0$ & $100 \cdot 0$ & $100 \cdot 0$ & $100 \cdot 0$ \\
\hline
\end{tabular}

It is easy to decide which of the acids is being dealt with by comparing the numbers which are obtained with those in Table B. Further, if a series of numbers corresponding to those given in Table $\mathrm{B}$ for acetic acid were obtained, then the amount of this acid in the original 110 c.c. can be got by looking up the factor corresponding to 100 c.c. (the volume distilled over). This factor is 80 (Table A), so it is only necessary to multiply the amount of acid found in 100 c.c. by $\frac{100}{80}=\frac{5}{4}$ to get the whole of the original quantity.

Where a mixture of two acids has to be dealt with, the conditions are somewhat more complicated, but both the acids can be estimated quantitatively if they have been determined qualitatively, as in Orla Jensen's method. It has already been mentioned that each acid on distillation behaves as though it alone were present. If, therefore, a mixture of equivalent quantities of two acids is present, the figures obtained by the method deseribed above are the arithmetical mean of the series of numbers given by the two acids in question. The curve 
which is thus obtained lies midway between the curves for the two acids. Where one acid is present in larger quantities than the other the curve for the mixed acids lies nearer to the curve for the predominating acid. To simplify these calculations Duclaux has drawn up a series of tables (see Table $\mathrm{X}$ in the Appendix) for different mixtures of fatty acids from $\mathrm{C}_{2}$ to $\mathrm{C}_{5}$.

It is evident that in mixtures of two acids the beginning and end of the curve must closely approach the curves for the single acids, so that it is enough to take the numbers for the 3rd, 4th, 5th, 6th, and 7 th fraction. When these data have been obtained, it is not difficult to determine the respective quantities of each of the acids present. With the help of table A (p. 222), it is first found in what proportions the two acids are present in the 10 fractions, and so the quantity of lime-water which would have been required to neutralise the mixture of acid originally present in the flask can be calculated. When this has been ascertained, and the strength of the limewater and the equivalent proportions of the two acids in the mixture being known, it is not difficult to calculate the absolute quantity of each of the two acids present in the mixture.

The practical carrying out of the method does not present any difficulties. First of all, the volume of milk is measured, and then without filtering, but after making acid with $\mathrm{H}_{2} \mathrm{SO}_{4}$, it is distilled with steam (p. 220). The steam is allowed to pass into the milk before the flask is heated, and so burning is prevented. When a sufficient quantity of distillate has been collected it is neutralised with lime water, the quantity required being noted, and the solution evaporated to less than 100 c.c. Afterwards, as much of a 10 per cent. solution of tartaric acid as is equivalent to the lime-water is added, and after cooling, the mixture is diluted to exactly 110 c.c. The amount of volatile fatty acid in this solution must not exceed 2 per cent. After 24 hours, the liquid is decanted from the crystals of calcium tartarate into a distillation flask, a few drops of concentrated sulphuric acid added to completely liberate the volatile fatty acid, and the distillation carried out in the usual way, fractions of 10 c.c. being collected. Some pieces of pumice-stone must be put into the liquid before distillation. The measuring flasks holding 10 c.c. are used as receivers. 
Two of them suffice, for whilst one is catching the distillate the contents of the other can be titrated.

The rate of distillation must be regulated-Duclaux advises 40-45 minutes-and when the distillation is over the relation between the total quantity of lime-water used and the quantity required for $10,20,30,40$ c.c. \&c., of the distillate is calculated from the successive readings on the burette. The series of numbers obtained in this way are compared with Table X in the Appendix; and this shows the mutual relation between the acids present in the mixture. The respective quantities are then calculated according to the method previously described.

Duclaux's method gives exact results when one, or at most two, acids are concerned. If only one acid is present, it can be estimated qualitatively or quantitatively by this method, but when the mixture of two acids has to be dealt with, Jensen's qualitative test must first be used, and the quantitative investigation performed according to Duclaux.

Often, however, several acids are present, and, if so, the fractions must be so divided that only two acids are present. Orla Jensen divides these fractions so that the precipitation of the barium salts can be made fractionally with normal sulphuric acid as already described. After each precipitation the barium sulphate is filtered off and washed, and the free acid distilled according to Duclaux's method. In this way the higher fatty acids are obtained in the first fraction, and the lower ones the last. The number of fractions depends upon the number and properties of the acids present. No generally applicable rule can be given, and in each case the procedure must be based upon the results of the qualitative examination. Acids which are only slightly soluble-caproic, and still more caprylic-cannot be determined with exactitude by Duclaux's method.

Further, as Orla Jensen ${ }^{1}$ has proved it is not, as regards the proportional number, a matter of indifference whether the acids are normal or isomerides, for when acids of the same molecular weight are present in aqueous solution, those which have the lowest boiling point, that is the "iso" acids, distil over first, and consequently higher numbers are oltained than for the

1 "Contribution à la connaissance et à l'analyse des acides gras volatils dans les huiles de palme et de beurre," Landwirtschaftliches Jahrbuch der Schweiz, 1905. 
corresponding normal acids. Orla Jensen found the following proportional numbers for butyric, valeric acids and their isomerides :-

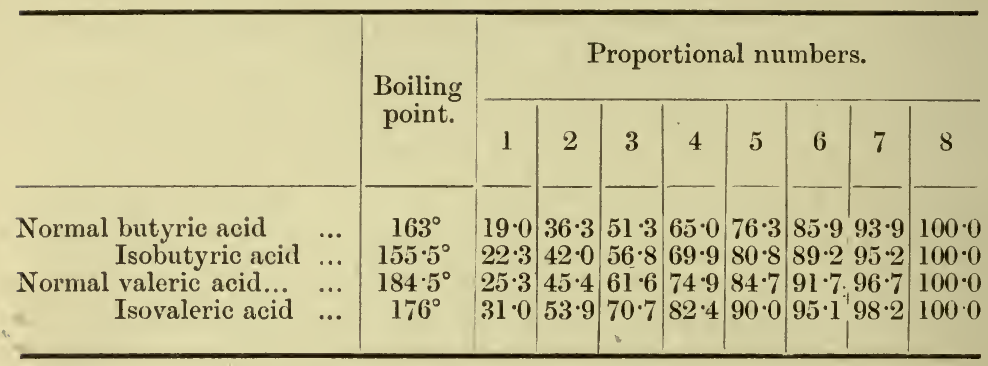

The proportional numbers for normal butyric acic and isovaleric acid agree with those given by Duclaux for the same acids. As is seen, the figures relating to the normal acids are considerably lower than those for the corresponding "iso" acids, so that Duclaux's method can serve to separate an acid from the "iso" acid, and for this purpose is the best method when a single acid in a pure state is in question.

\section{Alcohol.}

In the normal spontaneous decomposition of milk, little or no alcohol is formed, but where a special fermentation takes place, as with koumiss or kephir, considerable quantities may arise.

The test usually employed, or at least usually recommended, for the detection of small quantities of alcohol, is the iodoform test. The filtered milk is made alkaline with milk of lime, 100 c.c. distilled, and the first 10 c.c. of the distillate warmed in a test-tube in the water-bath to $60^{\circ}-70^{\circ} \mathrm{C}$. A little caustic soda and a few grains of iodine are then added, excess of alkali being avoided, as it makes the test less sensitive. After the tube has been well shaken to dissolve the iodine, it is cooled, and the presence of alcohol is denoted by a yellow precipitate of iodoform. This precipitate can be identified by the characteristic iodoform smell, and by the microscopic examination in which the iodoform crystals are seen to be regular hexagonal flakes. 
The reaction takes place according to the following equation :-

$$
\mathrm{C}_{2} \mathrm{H}_{5} \mathrm{OH}+6 \mathrm{NaOH}+8 \mathrm{I}=5 \mathrm{H}_{2} \mathrm{O}+5 \mathrm{NaI}+\mathrm{HCOONa}+\mathrm{CHI}_{3} \text {. }
$$

Although this reaction is very sensitive, it has the disadvantage that it takes place, not only with alcohol, but also with a whole series of other substances, such as acetone, aldehyde, grape sugar, lactic acid, substances which contain the group $\mathrm{CH}_{2}-\mathrm{CO}$. Lactic acid is not, as was formerly thought, an entirely non-volatile acid, but is partly volatile. A feeble reaction is therefore not a proof that alcohol is really present. Perfectly fresh drawn normal milk also gives a distinct but faint iodoform reaction, as the author has repeatedly proved.

Another qualitative method for detecting alcohol consists in adding dilute sulphuric acid to the distillate mentioned above, and then a small crystal of potassium bichromate. On warming gently, acetaldehyde, $\mathrm{CH}_{3} \cdot \mathrm{CHO}$, is given off, which blackens a strip of filter-paper moistened with an ammoniacal silver solution. ${ }^{1}$

A third method is to shake the liquid with benzoyl chloride and caustic soda, which causes the formation of ethyl benzoate, characterised by its odour, if alcohol is present.

Duclaux ${ }^{2}$ has devised a quantitative method for the determination of small quantities of alcohol, which is based upon the smaller surface tension of alcohol as compared with water. Mixtures of alcohol and water have naturally a lower surface tension than pure water. If, therefore, a given volume of alcohol and water is allowed to flow from a capillary opening, the number of drops at a given temperature is constant for the mixture, and with an increasing proportion of alcohol the number of drops increases. Duclaux makes use of a so-called "compte-gouttes" (a drop counter) which consists of a 5 c.c. pipette provided with a capillary tube sufficiently long to allow of the liquid issuing in drops. The capillary opening is so adjusted that when the pipette is filled with water at $15^{\circ} \mathrm{C}$. it empties in 100 drops. When an alcohol-containing liquid is to be tested, the pipette is filled to the mark, the outlet, which must be free from every trace of fat, carefully cleaned with filter-paper, and the pipette placed in a suitable holder. The

1 See under " Determination of Degree of Oxidation of Butter," p. 232.

2 Traité de Microbiologie. Vol. III, p. 7. 
liquid is then allowed to flow out, and the drops are counted. Duclaux has arranged the following table, which shows the percentage of alcohol in a liquid according to the number of drops at different temperatures :-

\begin{tabular}{|c|c|c|c|c|c|c|c|c|c|c|}
\hline \multicolumn{3}{|c|}{ Temp. ${ }^{\circ} \mathrm{C}$} & $5^{\circ}$ & $7 \cdot 5^{\circ}$ & $10^{\circ}$ & $12 \cdot 5^{\circ}$ & $15^{\circ}$ & $17 \cdot 5^{\circ}$ & $20^{\circ}$ & $22 \cdot 5^{\circ}$ \\
\hline \multicolumn{3}{|c|}{ Dist. Water } & 98 & $98 \cdot 5$ & 99 & $99 \cdot 5$ & 100 & $100 \cdot 5$ & 101 & 102 \\
\hline $\begin{array}{c}\text { Alcohe } \\
\quad, \\
\text { ", } \\
\text {," } \\
\text {," } \\
\text { ", } \\
\text { ", } \\
\text {," } \\
\text { ", }\end{array}$ & $\begin{array}{c}\text { ol } 0.5 \\
1 \\
2 \\
3 \\
4 \\
5 \\
6 \\
7 \\
8 \\
9 \\
10 \\
11 \\
12 \\
13 \\
14 \\
15\end{array}$ & $\begin{array}{l}\text { er cent. } \\
\text {, } \\
, \\
, \\
, \\
, \\
, \\
, \\
, \\
, \\
, \\
, \\
, \\
, \\
,\end{array}$ & $\begin{array}{l}102 \\
105 \\
111 \\
116 \\
120 \cdot 5 \\
124 \\
127 \\
130 \\
133 \\
136 \\
139 \\
142 \\
145 \\
148 \cdot 5 \\
152 \\
155\end{array}$ & $\begin{array}{l}102 \cdot 5 \\
105 \cdot 5 \\
111 \cdot 5 \\
116 \cdot 5 \\
121 \\
124 \cdot 5 \\
127 \cdot 5 \\
131 \\
134 \\
137 \\
140 \cdot 5 \\
143 \cdot 5 \\
146 \cdot 5 \\
150 \\
153 \cdot 5 \\
156 \cdot 5\end{array}$ & $\begin{array}{l}103 \\
106 \\
112 \\
117 \\
121 \cdot 5 \\
125 \\
128 \cdot 5 \\
132 \\
135 \cdot 5 \\
138 \cdot 5 \\
141 \cdot 5 \\
144 \cdot 5 \\
148 \\
151 \\
154 \cdot 5 \\
157 \cdot 5\end{array}$ & $\begin{array}{l}103 \cdot 5 \\
106 \cdot 5 \\
112 \cdot 5 \\
117 \cdot 5 \\
122 \\
125 \cdot 5 \\
129 \cdot 5 \\
133 \\
136 \cdot 5 \\
139 \cdot 5 \\
142 \cdot 5 \\
145 \cdot 5 \\
149 \\
152 \cdot 5 \\
155 \cdot 5 \\
158 \cdot 5\end{array}$ & $\begin{array}{l}104 \\
107 \\
113 \\
118 \\
122 \cdot 5 \\
126 \cdot 5 \\
130 \cdot 5 \\
134 \\
137 \cdot 5 \\
140 \cdot 5 \\
144 \\
147 \\
150 \cdot 5 \\
154 \\
157 \\
160\end{array}$ & $\begin{array}{l}104 \cdot 5 \\
107 \cdot 5 \\
113 \cdot 5 \\
118 \cdot 5 \\
123 \cdot 5 \\
127 \cdot 5 \\
131 \cdot 5 \\
135 \cdot 5 \\
139 \\
142 \\
145 \\
148 \\
151 \cdot 5 \\
155 \\
158 \\
161 \cdot 5\end{array}$ & $\begin{array}{l}105 \\
108 \\
114 \cdot 5 \\
119 \cdot 5 \\
124 \cdot 5 \\
128 \cdot 5 \\
132 \cdot 5 \\
136 \cdot 5 \\
140 \\
143 \\
146 \cdot 5 \\
149 \cdot 5 \\
153 \\
156 \\
159 \\
163\end{array}$ & $\begin{array}{l}106 \\
109 \\
115 \cdot 5 \\
120 \cdot 5 \\
125 \cdot 5 \\
130 \\
134 \\
135 \\
141 \cdot 5 \\
144 \cdot 5 \\
147 \cdot 5 \\
150 \cdot 5 \\
154 \cdot 5 \\
157 \cdot 5 \\
160 \cdot 5 \\
164 \cdot 5\end{array}$ \\
\hline
\end{tabular}

When the amount of alcohol reaches about 15 per cent., the method loses its sensitiveness, and it is then better to determine the specific gravity with the pyknometer. With small quantities of alcohol, however, Duclaux's method is more accurate than any other. Between pure water and a 1 per cent. alcohol solution there is a difference of 7 drops, as may be seen in the table; the apparatus is accurate to half a drop. The drawback to the method is that other volatile substances which distil over with the alcohol may increase the number of drops. Where great exactness is wished, both Duclaux's method and that of the specific gravity can be used to check one another. After the determination of the specific gravity has been made, the percentage of alcohol is easily ascertained from tables prepared for this purpose, and which are to be found in every treatise dealing with the analysis of foods.

As regards the testing for and estimation of other decomposition products of milk, such as aldehyde, ${ }^{1}$ acetone, peptone

1 For the detection of aldehyde, see p. 232. 
leucine, tyrosine, phenol, indol, skatcl, \&c., the reader is referred to text-books on organic chemistry, for the methods are only exceptionally carried out in practical analytical laboratories.

\section{II.-Decomposition Products of Butter.}

The normal spontaneous decomposition of butter is seen in every day life when butter turns rancid. This process consists in the continued splitting up of the glycerides of the butter into glycerine and free fatty acids. These free fatty acids, the volatile ones, and particularly butyric acid, impart the objectionable rancid taste and smell. This hydrolytic cleavage is caused by certain micro-organisms, and the part which they play in turning butter rancid has been proved by Orla Jensen. ${ }^{1}$ The most important of these micro-organisms are the following:-Oidium lactis, Cladosporium butyri (both of which are moulds), also Bacillus fluorescens liquefaciens, and less often Bacterium prodigiosum.

Ö̈dium lactis, Bacillus fluorescens liquefaciens, and Bacterium prodigiosum can only split up the fat, and are not able to attack the combined glycerine. They split up the butter fat without the formation of esters. Cladosporium butyri and Penicillium glaucum not only split up the fat, but also attack the glycerine. They split up the butter fat with the formation of transitory esters. Finally, certain varieties of mycoderma have not the power to split up the fat, but they can attack the combined glycerine. They form only esters. Amongst the esters the first place is taken by ethyl butyrate, and its importance in the taste and smell of rancid butter has been investigated by Amthor. ${ }^{2}$

In this way butter undergoes total decomposition due to the large number of micro-organisms (many millions per gram immediately after the butter has been made) and to the quantity of food in the form of casein, milk sugar, and salts which is at their disposal. The larger the amount of such substances in the butter the more rapid is the decomposition.

1 "Studien über das Ranzigwerden der Butter," Landwirtschaftliches Jahrbuch der Schueiz, 1901.

${ }_{2}$ Zeitschrift für analytische Chemie, 1899, p. 10. 
The process of becoming rancid spreads from the outside to the interior in the same way as the ripening in soft cheese.

The extent of hydrolysis is simply determined by estimating the amount of free acid by titration. There exists, however, no definite relation between the degree of rancidity and the amount of free fatty acids.

Direct sunlight causes butter fat (like other fats) to undergo another process of transformation, namely, oxidation, whereby the unsaturated fatty acids in the butter, that is the oleic acid principally, are attacked, as Duclaux and others have shown. The glycerine is also partially attacked. The advance in the process of oxidation is best measured by the iodine number, which of course diminishes more and more. It is further of interest to test for aldehyde, which is also formed, and which bleaches the butter, and gives it at the same time a tallowy, not rarid, taste. This process is distinguished from the biological decomposition which results in the butter becoming rancid. The difference between the two processes is shown in the following table by Orla Jensen :-

\begin{tabular}{|c|c|c|c|c|c|c|c|}
\hline & Colour. & $\begin{array}{l}\text { Sinell } \\
\text { and } \\
\text { taste. }\end{array}$ & $\begin{array}{l}\text { Decrease } \\
\text { of iodine } \\
\text { number. }\end{array}$ & $\begin{array}{l}\text { Alde- } \\
\text { hyde } \\
\text { reaction. }\end{array}$ & $\begin{array}{l}\text { Increase } \\
\text { of acid } \\
\text { number. }\end{array}$ & $\begin{array}{l}\text { The free } \\
\text { fatty } \\
\text { acids are }\end{array}$ & $\begin{array}{l}\text { Butyric } \\
\text { acid } \\
\text { ester. }\end{array}$ \\
\hline $\begin{array}{c}\text { Butter } \\
\text { decomposed } \\
\text { in sunlight }\end{array}$ & $\begin{array}{c}\text { Un- } \\
\text { changed } \\
\text { White }\end{array}$ & Tallowy & $\begin{array}{c}\text { Little or } \\
\text { none. } \\
\text { Large }\end{array}$ & $\begin{array}{l}\text { Weak } \\
\text { Strong }\end{array}$ & $\begin{array}{l}\text { Large } \\
\text { Little }\end{array}$ & $\begin{array}{l}\text { Princi- } \\
\text { pally } \\
\text { non- } \\
\text { volatile } \\
\text { Princi- } \\
\text { pally } \\
\text { volatile }\end{array}$ & + \\
\hline
\end{tabular}

\section{Determination of the Degree of Rancidity.}

The extent of the hydrolytic processes going on in butter can be measured, as mentioned above, by titrating the amount of free acid. In this way the acid number is obtained expressed as the number of cubic centimetres of normal alkali required to neutralise $100 \mathrm{~g}$. of fat. The method of making this determination has alrcady been given on p. 156. The acid number of butter does not in any way serve as a measure of those products of rancidity which affect the taste and smell. 
A more accurate idea of the degree of rancidity of butter is obtained by distillation of the volatile fatty acids of the butter fat, and the amount of these, as well as of the esters, determined. Amthor ${ }^{1}$ employs the following method for this purpose:

Ten g. of butter are distilled with steam until 500 c.c. of distillate are collected. The volatile fatty acids contained in the distillate are titrated with $N / 10$ alkali and then 50 c.c. of $N / 10$ alkali are added, the mixture heated for half an hour with a reflux condenser, and the free alkali titrated back. The difference gives the amount of alkali required to saponify the ester-like substances. Amthor calls the number of c.c. of $N / 10$ alkali required to saponify the ester-like substances from $100 \mathrm{~g}$. of butter the "ester number."

The fact that normal butter also contains volatile substances which are saponifiable with caustic potash makes it impossible, as Amthor himself agrees, to regard the ester number as strictly quantitative, and this necessitates a determination of the butyric acid ester.

Orla Jensen ${ }^{2}$ has also proved that the more or less pronounced smell of the ester which is developed in butter is not always in proportion to the quantity of volatile, saponifiable substances. He therefore disregards quantitative determinations, and contents himself with the "total acid number" on the one hand, and on the other with the "volatile acid number," by which last he understands the number of c.c. of normal alkali which are required to neutralise the distillate from $100 \mathrm{~g}$. of butter. Jensen employs Amthor's method of distillation, and in addition washes out the condenser at the end of the operation with 100 c.c. neutral absolute alcohol, in order to get the higher soluble fatty acids. Where the decomposition of the butter is far advanced, more alcohol is needed to bring all the fatty acids into solution before titrating.

It is important to notice that Orla Jensen has proved that, although the free volatile fatty acids in rancid butter only make up a small part of the total free fatty acids, the conclusion cannot be drawn that the glycerides of the non-volatile acids are attacked more readily by the micro-organisms than are the glycerides of

$$
{ }^{1} \text { Ref., p. } 229 .
$$




\section{MILK AND DAIRY PRODUCTS}

the volatile fatty acids. The fact really is, as Hanus and Stocky ${ }^{1}$ have proved, that the volatile acirls freed by hydrolytic cleavage are attacked more greedily by the micro-organisms. Likewise, the greater part of the free volatile fatty acids fail to be estimated because they, more than the solid acids, unite with the ammonia present in the drops of brine of the butter.

Immediately after the butter has been made, the "total acid number" generally varies between 1 and 3 . In very rancid butter the number may rise to 20 or 30 in the exterior portions, whilst in the interior it may be about 10 . The "volatile acid number" immediately after the butter has been made $=0$, or, at most, $\cdot 1-2$, whilst in the outer portion of a very rancid butter the number never exceeds 1 .

\section{Determination of the Degree of Oxidation.}

As already mentioned, the oleic acid in butter is oxidised under the action of sunlight, and imparts a tallowy flavour. A determination of the iodine number (p. 153) serves as a measure of the advance of the process of oxidation. Not much use, however, can be made of this determination unless the figure obtained is very low, for the iodine number is known to vary within wide limits, even with normal butter.

On this account, the distillate from butter must be tested also for aldehyde, either by the ammoniacal silver test or some other reagent. By operating in the following manner, the sensitiveness of the ammoniacal silver reaction can be increased. A few drops of caustic potash or caustic soda solution are added to the silver nitrate solution, and the precipitated $\mathrm{Ag}_{2} \mathrm{O}$ afterwards dissolved in ammonia. The solution obtained in this way is used for the test.

Schiff s reagent, which is a solution of fuchsin that has been decolorised by sulphur dioxide, is also used as a test for aldehyde. The reagent becomes violet in presence of aldehyde, and the test is made particularly sensitive by following Gayon's ${ }^{2}$ directions, which are to mix

1000 c.c. aqueous fuchsin solution, $1: 1000$.

20 "sodium bisulphite, $30^{\circ} \mathrm{B}$.

10 "pure concentrated hydrochloric acid.

1 Zeitschrift für Untersuchung der Nahr.-und Genussmittel, 1900, No. 19.

${ }^{2}$ Compt. rend. de l'A cad. des Sciences, Vol. IV, 1887. 
The bisulphite solution is added to the fuschin solution, and an hour later, when the decolorisation is almost complete, the hydrochloric acid is added. The test is carried out by shaking $2 \mathrm{c} \mathrm{c}$ of the distillate from the butter with 1 c.c. of the reagent and then allowing the mixture to stand. In the presence of aldehyde, a violet colour, which gradually deepens, soon becomes apparent.

\section{III.-Decomposition Products of Cheese.}

\section{Introductory.}

The decomposition of cheese, which is known as "ripening," is a process which can be modified artificially in certain directions, and is more or less thorough according to the kind of cheese which is being made. The main features of the chemical changes involved in ripening have already been sketched in the introduction to the chapter on the analysis of cheese (p. 182). ${ }^{1}$

\section{Determination of the Degree of Acidity.}

In this determination the cheese itself must be titrated, and not an aqueous extract of it, for the acids arising from the fermentation of the milk sugar are mainly in combination with the lime of the phosphate and the paracasein. ${ }^{2}$

Ten g. of cheese are well ground in a mortar with water at $40^{\circ}-45^{\circ} \mathrm{C}$. The emulsion is diluted to 100 c.c. and titrated with $N / 4$ alkali, phenolphthalein being used as indicator. The number of cubic centimetres of $N / 4$ alkali is multiplied by 10 , and gives the degree of acidity of the cheese. This determination is analogous to the one for the degree of acidity of milk.

\section{Volatile Fatty Acids.}

The volatile fatty acids in cheese are determined in the same way as those of milk (p. 219). One hundred g. of an average

I A detailed description of the bacteriological processes which go on in milk and dairy products will be found in the author's Bakteriologie des Meirereiwesens, published by M. Heinsius Nachfolger, Leipsic.

2 Orla Jensen, "Ueber die im Finmentaler Käse stattfindende Milchsäuregärung, Landwirt. Jahrbuch der Schweiz, 1906. 
sample of cheese are treated with 200 c.c. of boiled water and a few c.c. of sulphuric acid, and then distilled with steam. Just as in the case of milk, the steam must be passed into the flask before the direct heating begins, so as to prevent the contents from burning. The distillation is carried on until a litre of distillate has been collected, and this is titrated with baryta water, evaporated, and treated, as on p. 220.

\section{Acid Number of Cheese Fat.}

The acid number of cheese fat is the number of cubic centimetres of standard alkali which are needed to neutralise $100 \mathrm{~g}$ of fat.

In order to separate the fat, ${ }^{1}$ the cheese is heated in the same way as in the estimation of fat by the Schmid-Bondzynski method (p. 187), that is, with hydrochloric acid (sp. gr. 1·1), in a boiling water-bath until the protein dissolves completely. After the burner has been taken from under the water-bath, the beaker is allowed to remain for half an hour to permit the fat to collect on the surface of the liquid. After this, the beaker is cooled in ice-water and the solid cake of fat subsequently washed with cold water. This cake of acids is then melted with water, and again allowed to cool. The melting and freezing of the solid acids is continued until the wash-water shows no trace of hydrochloric acid. Finally, the cake of fat is dried between filter-paper, dried, melted, and filtered. ${ }^{2}$

The degree of acid of the fat obtained by the hydrochloric acid method serves as a measure of the decomposition which the fat has undergone in the cheese. ${ }^{3}$

\section{Nitrogenous Substances.}

In order to get a general idea of the degree of decomposition of paracasein in cheese, it is generally sufficient to determine the total nitrogen, the soluble nitrogen, and the ammoniacal nitrogen. The best way of making these analyses has already

${ }^{1}$ K. Windisch, "Über Margarine Käse," Arbeiten aus dem Kaiserlichєn Gesundheitsamte, Berlin, 1898, p. 554.

2 If it is not possible to get a solid cake of fat, then the fat can be shaken out with ether.

der Schweiz, 1904, p. 326. 
been given in the section dealing with the examination of cheese.

It would be beyond the scope of this work to give methods for estimating the amount of the numerous and varying substances which arise during ripening of the protein of the cheese. Such estimations necessitate a thorough scientific treatment, for reliable methods are not to be found in this branch of the subject. Special attention is, however, directed to the article, "Zur Kenntniss der Bestandteile des Emmentaler Käses," by E. Winterstein and W. Bissegger, published in Hoppe-Seyler's Zeitschrift fiur physiologische Chemie, Vol. XLVII, 1906, p. 28. 


\section{APPENDIX}

Table I.-lior Cokrecting Lactometer Readings at Different Temperatures, according to Cirr. Müller.

(a) In the case of Whole Milk.

\begin{tabular}{|c|c|c|c|c|c|c|c|c|c|c|c|}
\hline \multirow{2}{*}{$\begin{array}{c}\text { Lactometer } \\
\text { degrees. }\end{array}$} & \multicolumn{11}{|c|}{ Temperature of Milk in degrees centigrade. } \\
\hline & 10 & 11 & 12 & 13 & 14 & 15 & 16 & 17 & 18 & 19 & 20 \\
\hline 14 & $13 \cdot 4$ & $3 \cdot 5$ & $13 \cdot 6$ & $13 \cdot 7$ & $13 \cdot 8$ & $14 \cdot 0$ & $14 \cdot 1$ & $14 \cdot 2$ & $14 \cdot 4$ & $14 \cdot 6$ & 14 \\
\hline & $14 \cdot 4$ & $14: 5$ & $14 \cdot 6$ & $14 \cdot 7$ & & & & & & $5 \cdot 6$ & $15 \cdot 8$ \\
\hline 16 & $15 \cdot 4$ & $15 \cdot 5$ & $15 \cdot 6$ & $15 \cdot 7$ & 15.8 & $16 \cdot 0$ & $16 \cdot 1$ & $16 \cdot 3$ & 16.5 & $36 \cdot 7$ & 16 \\
\hline 17 & $16 \cdot 4$ & 16.5 & $16 \cdot 6$ & 16.7 & $16 \cdot 8$ & $17 \cdot 0$ & $17 \cdot 1$ & $17 \cdot 3$ & $17 \cdot 5$ & $17 \cdot 7$ & 17 \\
\hline & $17 \cdot 4$ & $17 \cdot 5$ & $17 \cdot 6$ & $17 \cdot 7$ & $17 \cdot 8$ & $18 \cdot 0$ & $18 \cdot 1$ & $18 \cdot 3$ & $18 \cdot 5$ & 18.7 & 18 \\
\hline 19 & $18 \cdot 4$ & $18 \cdot 5$ & $18 \cdot 6$ & $18 \cdot 7$ & $18 \cdot 8$ & $19^{\circ} 0$ & $19 \cdot 1$ & $19 \cdot 3$ & 19.5 & $19 \cdot 7$ & 19 \\
\hline & & $19 \cdot 4$ & 19.5 & $19 \cdot 6$ & $19 \cdot 8$ & $20 \cdot 0$ & $20 \cdot 1$ & $20 \cdot 3$ & $20 \cdot 5$ & $20 \cdot 7$ & $20 \cdot 9$ \\
\hline & $20 \cdot 3$ & $20 \cdot 4$ & 20.5 & $20 \cdot 6$ & 20.8 & $21 \cdot 0$ & $21 \cdot 2$ & $21 \cdot 4$ & $21 \cdot 6$ & 21.8 & $22 \cdot 0$ \\
\hline 2 & $21 \cdot 3$ & $21 \cdot 4$ & 21.5 & $21 \cdot 6$ & $21 \cdot 8$ & $22 \cdot 0$ & $22 \cdot 2$ & $22 \cdot 4$ & $22 \cdot 6$ & $22 \cdot 8$ & $23 \cdot 0$ \\
\hline & $22 \cdot 3$ & $22 \cdot 4$ & 2.5 & $22 \cdot 6$ & $22 \cdot 8$ & $23 \cdot 0$ & $23 \cdot 2$ & $23 \cdot 4$ & $23 \cdot 6$ & $23 \cdot 8$ & $24 \cdot 0$ \\
\hline & $23 \cdot 3$ & $23 \cdot 4$ & $23 \cdot 5$ & $23 \cdot 6$ & 23.8 & $24 \cdot 0$ & $24 \cdot 2$ & $24 \cdot 4$ & $24^{\circ} 6$ & $24 \cdot 8$ & $25 \cdot 0$ \\
\hline & $24 \cdot 2$ & $24 \cdot 3$ & $4 \cdot 5$ & $24 \cdot 6$ & $24 \cdot 8$ & $25^{\circ} 0$ & $25 \cdot 2$ & $25 \cdot 4$ & $25 \cdot 6$ & $25 \cdot 8$ & $26 \cdot 0$ \\
\hline & $25 \cdot 2$ & 25 & $5 \cdot 5$ & $25 \cdot 6$ & $25 \cdot 8$ & $26 \cdot 0$ & $26 \cdot 2$ & $26 \cdot 4$ & $26 \cdot 6$ & $26 \cdot 9$ & $27 \cdot 1$ \\
\hline & $26 \cdot 2$ & $26 \cdot 3$ & $6 \cdot 5$ & $26 \cdot 6$ & 26.8 & $27 \cdot 0$ & $27 \cdot 2$ & $27 \cdot 4$ & $27 \cdot 6$ & $27 \cdot 9$ & $28 \cdot 2$ \\
\hline & $27 \cdot 1$ & $27 \cdot 2$ & 4 & $27 \cdot 6$ & $27 \cdot 8$ & $28 \cdot 0$ & $28 \cdot 2$ & $28 \cdot 4$ & $28 \cdot 6$ & $28 \cdot 9$ & $29 \cdot 2$ \\
\hline & $28 \cdot 1$ & $28 \cdot 2$ & $28 \cdot 4$ & $28 \cdot 6$ & $28 \cdot 8$ & $29 \cdot 0$ & $29 \cdot 2$ & $29 \cdot 4$ & $29 \cdot 6$ & $29 \cdot 9$ & $30 \div 2$ \\
\hline & $29 \cdot 0$ & $29 \cdot 2$ & $9 \cdot 4$ & $29 \cdot 6$ & $29 \cdot 8$ & 30.0 & $30 \cdot 2$ & 30.4 & 30.6 & $30 \cdot 9$ & $31{ }^{\prime} 2$ \\
\hline & $\cdot 0$ & $30 \cdot 2$ & $30 \cdot 4$ & $30 \cdot 6$ & $30 \cdot 8$ & $31 \cdot 0$ & $31 \cdot 2$ & $31 \cdot 4$ & $31 \cdot 7$ & $32 \cdot 0$ & $32 \cdot 3$ \\
\hline 3 & 31.0 & $31 \cdot 2$ & $31 \cdot 4$ & $31 \cdot 6$ & $31 \cdot 8$ & $32 \cdot 0$ & $32 \cdot 2$ & $32 \cdot 4$ & $32 \cdot 7$ & 33.0 & $33 \cdot 3$ \\
\hline & & $32 \cdot 2$ & $32 \cdot 4$ & $32 \cdot 6$ & $32 \cdot 8$ & $33 \cdot 0$ & $33 \cdot 2$ & & $33 \cdot 7$ & $34^{\circ} 0$ & $34 \cdot 3$ \\
\hline & 32.9 & $33 \cdot 1$ & 33 & $33 \cdot 5$ & $33 \cdot 8$ & $34 \cdot 0$ & $34 \cdot 2$ & 34 & $34 \cdot 7$ & $35 \cdot 0$ & $35 \cdot 3$ \\
\hline שJ & $33 \cdot 8$ & $34 \cdot 0$ & $34 \cdot 2$ & $34 \cdot 4$ & $34 \cdot 7$ & $35 \cdot 0$ & $35 \cdot 2$ & $35 \cdot 4$ & $35 \cdot 7$ & 36.0 & $36 \cdot 3$ \\
\hline
\end{tabular}


TAkte I-Continued.

(b) In the case of Seprarated Milk.

\begin{tabular}{|c|c|c|c|c|c|c|c|c|c|c|c|c|}
\hline \multirow{2}{*}{$\begin{array}{l}\text { Lacto- } \\
\text { meter } \\
\text { de- } \\
\text { grees. }\end{array}$} & \multicolumn{12}{|c|}{ Temperature of Milk in degrees centigrade. } \\
\hline & 10 & 11 & 12 & 13 & 14 & 15 & 16 & 17 & 18 & 19 & 20 & 21 \\
\hline 18 & $17 \cdot 5$ & $7 \cdot 6$ & $17 \cdot 7$ & $17 \cdot 8$ & $17 \cdot 9$ & $18 \cdot 0$ & $18 \cdot 1$ & $18 \cdot 2$ & 18.4 & $18 \cdot 6$ & $18 \cdot 8$ & 18.8 \\
\hline 19 & 18.5 & 18.6 & $18 \cdot 7$ & 18.8 & 18.9 & 19.0 & $19 \cdot 1$ & $19 \cdot 2$ & $19 \cdot 4$ & 19.6 & 19.8 & 10. \\
\hline 20 & 19.5 & $19 \cdot 6$ & 19.7 & 19.8 & 19.9 & $20 \cdot 0$ & $20 \cdot 1$ & $20 \cdot 2$ & & $20 \cdot 6$ & $20 \cdot 8$ & 20.9 \\
\hline 21 & 20.5 & $20 \cdot 6$ & 20.7 & $20 \cdot 8$ & 20.9 & $21 \cdot 0$ & $21 \cdot 1$ & $21 \cdot 2$ & $21 \cdot 4$ & $21 \cdot 6$ & $21 \cdot 8$ & $21 \cdot 9$ \\
\hline 22 & 21.5 & $21 \cdot 6$ & $21 \cdot 7$ & $21 \cdot 8$ & $21 \cdot 9$ & 22.0 & $22 \cdot 1$ & $22 \cdot 2$ & $22 \cdot 4$ & $22 \cdot 6$ & 22.8 & 22.9 \\
\hline 23 & $22 \cdot 5$ & $22 \cdot 6$ & $22 \cdot 7$ & $22 \cdot 8$ & 22.9 & 23.0 & $23 \cdot 1$ & $23 \cdot 2$ & $23 \cdot 4$ & $23 \cdot 6$ & 23.8 & 23.9 \\
\hline 24 & $23 \cdot 4$ & $23 \cdot 5$ & $23 \cdot 6$ & $23 \cdot 7$ & 23.9 & $24 \cdot 0$ & $24 \cdot 1$ & $24 \cdot 2$ & $24 \cdot 4$ & $24 \cdot 6$ & $24 \cdot 8$ & 24.9 \\
\hline 25 & $24 \cdot 3$ & $24 \cdot 4$ & $24 \cdot 5$ & $24 \cdot 6$ & $24 \cdot 8$ & $25 \cdot 0$ & $25 \cdot 1$ & $25 \cdot 2$ & $25 \cdot 4$ & $25 \cdot 6$ & 25.8 & $25 \cdot 9$ \\
\hline 26 & $25 \cdot 3$ & 25.4 & 25.5 & $25 \cdot 6$ & $25 \cdot 8$ & 26.0 & $26 \cdot 1$ & 26.3 & 26.5 & $26 \cdot 7$ & 26.9 & 27.0 \\
\hline 27 & $26 \cdot 3$ & $26 \cdot 4$ & 26.5 & $26 \cdot 6$ & $26 \cdot 8$ & $27 \cdot 0$ & $27 \cdot 1$ & $27 \cdot 3$ & $27 \cdot 5$ & $27 \cdot 7$ & $27 \cdot 9$ & $28 \cdot 1$ \\
\hline 28 & $27 \cdot 3$ & $27 \cdot 4$ & $27 \cdot 5$ & $27 \cdot 6$ & $27 \cdot 8$ & $28 \cdot 0$ & $28 \cdot 1$ & $28 \cdot 3$ & $28 \cdot 5$ & 28.7 & $28 \cdot 9$ & $29 \cdot 1$ \\
\hline 29 & $28 \cdot 3$ & $28 \cdot 4$ & $8 \cdot 5$ & $28 \cdot 6$ & $28 \cdot 8$ & $29 \cdot 0$ & $29 \cdot 1$ & $29 \cdot 3$ & 29.5 & $29 \cdot 7$ & $29 \cdot 9$ & $30 \cdot 1$ \\
\hline 30 & $29 \cdot 3$ & $29 \cdot 4$ & $29 \cdot 5$ & $29 \cdot 6$ & 29.8 & 30.0 & $30 \cdot 1$ & $30 \cdot 3$ & 30.5 & 30.7 & 30.9 & $31 \cdot 1$ \\
\hline 31 & $30: 3$ & 30.4 & 30.5 & $30 \cdot 6$ & $30 \cdot 8$ & $31 \cdot 0$ & $31 \cdot 2$ & 31 & $31 \cdot 6$ & 3 & $32 \cdot 0$ & $32 \cdot 2$ \\
\hline 32 & $31 \cdot 3$ & $31 \cdot 4$ & 31.5 & $31 \cdot 6$ & $31 \cdot 8$ & $32 \cdot 0$ & $32 \cdot 2$ & $32 \cdot 4$ & $32 \cdot 6$ & 32.8 & $33 \cdot 0$ & $33 \cdot 2$ \\
\hline 33 & $32 \cdot 3$ & $32 \cdot 4$ & $2 \cdot 5$ & $32 \cdot 6$ & $32 \cdot 8$ & $33 \cdot 0$ & $33 \cdot 2$ & $33 \cdot 4$ & $33 \cdot 6$ & $33 \cdot 8$ & $34 \cdot 0$ & $34 \cdot 2$ \\
\hline 34 & $33 \cdot 3$ & 33.4 & 33.5 & 38 & 33.8 & $34 \cdot 0$ & $34 \cdot 2$ & $34 \cdot 4$ & 3 & 3 & $35 \cdot 0$ & $35^{\circ}$ \\
\hline 35 & $34 \cdot 2$ & $34 \cdot 3$ & $34 \cdot 4$ & $34 \cdot 6$ & 34.8 & 35.0 & $35 \cdot 2$ & 35.4 & $35 \cdot 6$ & $35 \cdot 8$ & 36.0 & $36 \cdot 2$ \\
\hline 36 & $35 \cdot 2$ & $35 \cdot 3$ & 35.4 & $35 \cdot 6$ & 35 & 36.0 & $36 \cdot 2$ & $36 \cdot 4$ & $36 \cdot 6$ & 36.9 & $37 \cdot 1$ & 37.3 \\
\hline 3 & $36 \cdot 2$ & $36 \cdot 3$ & 36.4 & & $36 \cdot 8$ & $37 \cdot 0$ & 37 & 37 & $37 \cdot 6$ & 37.9 & $38 \cdot 2$ & $38 \cdot 4$ \\
\hline 38 & $37 \cdot 2$ & $37 \cdot 3$ & $37 \cdot 4$ & $37 \cdot 6$ & $37 \cdot 8$ & $38 \cdot 0$ & $38 \cdot 2$ & 38.4 & $38 \cdot 6$ & $38 \cdot 9$ & $39 \cdot \overline{2}$ & $39 \cdot 4$ \\
\hline 39 & $38 \cdot 2$ & $38 \cdot 3$ & $38 \cdot 4$ & 38.6 & 38 & $39 \cdot 0$ & $39 \cdot 2$ & $39 \cdot 4$ & $39 \cdot 6$ & 39.9 & $40 \cdot 2$ & $40 \cdot 4$ \\
\hline 40 & $39 \cdot 1$ & $39 \cdot 2$ & 39.4 & 39.6 & $39 \cdot 8$ & $40 \cdot 0$ & $40 \cdot 2$ & $40 \cdot 4$ & 40.6 & 40.9 & $41 \cdot 2$ & $41 \cdot 4$ \\
\hline
\end{tabular}




\section{MILK AND DAIRY PRODUCTS}

Table II.-For Estimating the Percentage of Fat in Milk from the Specific Gravity of the Ether-fat Soldtion at $17.5^{\circ} \mathrm{C}$, ACCORDING TO SOXHLET.

(a) Whole Milk.

\begin{tabular}{|c|c|c|c|c|c|c|c|c|c|}
\hline $\begin{array}{l}\text { Specific } \\
\text { gravity. }\end{array}$ & $\begin{array}{c}\text { Fat } \\
\%\end{array}$ & $\begin{array}{l}\text { Specific } \\
\text { gravity. }\end{array}$ & $\underset{\%}{\mathrm{Fat}}$ & $\begin{array}{l}\text { Specific } \\
\text { gravity. }\end{array}$ & $\begin{array}{c}\text { Fat } \\
\%\end{array}$ & $\begin{array}{l}\text { Specific } \\
\text { gravity. }\end{array}$ & $\begin{array}{c}\text { Fat } \\
\%\end{array}$ & $\begin{array}{l}\text { Specific } \\
\text { gravity. }\end{array}$ & $\underset{\%}{\text { Fat }}$ \\
\hline $43 \cdot 0$ & 2.07 & $47 \cdot 7$ & $2 \cdot 61$ & $52 \cdot 3$ & $3 \cdot 16$ & $56 \cdot 9$ & $3 \cdot 74$ & 61.5 & $4: 39$ \\
\hline$\cdot 1$ & 08 & .8 & $2 \cdot 62$ & 4 & $3 \cdot 17$ & $57 \cdot 0$ & 3.75 & 6 & $4 \cdot 40$ \\
\hline 2 & $2 \cdot 09$ & .9 & $2 \cdot 63$ & $\cdot 5$ & $3 \cdot 18$ & $\cdot 1$ & $3 \cdot 76$ & $\cdot 7$ & $4 \cdot 42$ \\
\hline$\cdot 3$ & $2 \cdot 10$ & $48^{\circ} 0$ & $2 \cdot 64$ & 6 & $3 \cdot 20$ & $\cdot 2$ & 3.78 & $\cdot 8$ & $4 \cdot 44$ \\
\hline$\cdot 4$ & $2 \cdot 11$ & $\cdot 1$ & $2 \cdot 66$ & $\cdot 7$ & $3 \cdot 21$ & $\cdot 3$ & $3 \cdot 80$ & $\cdot 9$ & $4 \cdot 46$ \\
\hline$\cdot \tilde{5}$ & $2 \cdot 12$ & $\cdot 2$ & $2 \cdot 67$ & 8 & $3 \cdot 22$ & $\cdot 4$ & $3 \cdot 81$ & $62 \cdot 0$ & $4 \cdot 47$ \\
\hline .6 & $2 \cdot 13$ & $\cdot 3$ & $2 \cdot 68$ & $\cdot 9$ & $3 \cdot 23$ & 5 & $3 \cdot 82$ & $\cdot 1$ & $4 \cdot 48$ \\
\hline$\cdot 7$ & $2 \cdot 14$ & $\cdot 4$ & $2 \cdot 70$ & $53 \cdot 0$ & $3 \cdot 25$ & 6 & $3 \cdot 84$ & $\cdot 2$ & $4 \cdot 50$ \\
\hline 8 & $2 \cdot 16$ & $\cdot 5$ & $2 \cdot 71$ & $\cdot 1$ & $3 \cdot 26$ & $\cdot 7$ & $3 \cdot 85$ & $\cdot 3$ & $4 \cdot 52$ \\
\hline .9 & $2 \cdot 17$ & 6 & $2 \cdot 72$ & $\cdot 2$ & $3 \cdot 27$ & .8 & 3.87 & $\cdot 4$ & 4.53 \\
\hline $44^{\circ} 0$ & $2 \cdot 18$ & .7 & $2 \cdot 73$ & $\cdot 3$ & $3 \cdot 28$ & $\cdot 9$ & $3 \cdot 88$ & $\cdot 5$ & $4 \cdot 55$ \\
\hline$\cdot 1$ & $2 \cdot 19$ & 8 & $2 \cdot 74$ & $\cdot 4$ & $3 \cdot 29$ & $58 \cdot 0$ & $3 \cdot 90$ & $\cdot 6$ & $4 \cdot 56$ \\
\hline$\cdot 2$ & $2 \cdot 20$ & 9 & $2 \cdot 75$ & .5 & $3 \cdot 30$ & $\cdot 1$ & 3.91 & $\cdot 7$ & $4 \cdot 58$ \\
\hline .3 & $2 \cdot 22$ & $49 \cdot 0$ & $2 \cdot 76$ & .6 & $3 \cdot 31$ & $\cdot 2$ & 3.92 & 8 & 4.59 \\
\hline$\cdot 4$ & $2 \cdot 23$ & $\cdot 1$ & $2 \cdot 77$ & $\cdot 7$ & $3 \cdot 33$ & 3 & 3.93 & $\cdot 9$ & $4 \cdot 61$ \\
\hline$\cdot 5$ & $2 \cdot 24$ & $\cdot 2$ & $2 \cdot 78$ & .8 & $3 \cdot 34$ & $\cdot 4$ & $3 \cdot 95$ & $63 \cdot 0$ & $4 \cdot 63$ \\
\hline .6 & $2 \cdot 25$ & $\cdot 3$ & $2 \cdot 79$ & .9 & $3 \cdot 35$ & $\cdot 5$ & 3.96 & $\cdot 1$ & $4 \cdot 64$ \\
\hline$\cdot 7$ & $2 \cdot 26$ & $\cdot 4$ & $2 \cdot 80$ & $54^{\circ} 0$ & $3 \cdot 37$ & 6 & $3 \cdot 98$ & $\cdot 2$ & $4 \cdot 66$ \\
\hline$\cdot 8$ & $2 \cdot 27$ & $\cdot 5$ & $2 \cdot 81$ & $\cdot 1$ & $3 \cdot 38$ & 7 & $3 \cdot 99$ & $\cdot 3$ & $4 \cdot 67$ \\
\hline .9 & $2 \cdot 28$ & $\cdot 6$ & $2 \cdot 83$ & .2 & $3 \cdot 39$ & .8 & $4 \cdot 01$ & $\cdot 4$ & $4 \cdot 69$ \\
\hline $45 \cdot 0$ & $2 \cdot 30$ & $\cdot 7$ & $2 \cdot 84$ & $\cdot \overline{3}$ & $3 \cdot 40$ & $\cdot 9$ & $4 \cdot 02$ & $\cdot \overline{5}$ & $4 \cdot 70$ \\
\hline$\cdot 1$ & $2 \cdot 31$ & $\cdot 8$ & $2 \cdot 86$ & $\cdot 4$ & $3 \cdot 41$ & $59 \cdot 0$ & $4 \cdot 03$ & $\cdot 6$ & 4.71 \\
\hline 2 & $2 \cdot 32$ & 9 & $2 \cdot 87$ & $\cdot 5$ & $3 \cdot 43$ & $\cdot 1$ & $4 \cdot 04$ & $\cdot 7$ & $4 \cdot 73$ \\
\hline .3 & $2 \cdot 33$ & $50 \cdot 0$ & $2 \cdot 88$ & .6 & $3 \cdot 45$ & .2 & $4 \cdot 06$ & 8 & $4 \cdot 75$ \\
\hline$\cdot 4$ & $2 \cdot 34$ & $\cdot 1$ & $2 \cdot 90$ & .7 & $3 \cdot 46$ & $\cdot 3$ & $4 \cdot 07$ & $\cdot 9$ & $4 \cdot 77$ \\
\hline$\cdot 5$ & $2 \cdot 35$ & $\cdot 2$ & $2 \cdot 91$ & .8 & $3 \cdot 47$ & 4 & $4 \cdot 09$ & $64^{\circ} \cdot 0$ & $4 \cdot 79$ \\
\hline$\cdot 6$ & $2 \cdot 36$ & $\cdot \overline{3}$ & $2 \cdot 92$ & $\cdot 9$ & 3.48 & $\cdot 5$ & $4 \cdot 11$ & $\cdot 1$ & $4 \cdot 80$ \\
\hline$\cdot 7$ & $2 \cdot 37$ & $\cdot 4$ & $2 \cdot 93$ & $55^{\circ} 0$ & $3 \cdot 49$ & 6 & $4 \cdot 12$ & 2 & 4.82 \\
\hline 8 & $2 \cdot 38$ & $\cdot 5$ & $2 \cdot 94$ & $\cdot 1$ & $3 \cdot 51$ & $\cdot 7$ & $4 \cdot 14$ & 3 & $4 \cdot 84$ \\
\hline$\cdot 9$ & $2 \cdot 39$ & $\cdot 6$ & $2 \cdot 96$ & $\cdot 2$ & $3 \cdot 52$ & $\cdot 8$ & $4 \cdot 15$ & $\cdot 4$ & 4.85 \\
\hline $46^{\circ} 0$ & $2 \cdot 40$ & $\cdot 7$ & $2 \cdot 97$ & $\cdot 3$ & 3.53 & .9 & $4 \cdot 16$ & .5 & $4 \cdot 87$ \\
\hline$\cdot 1$ & $2 \cdot 42$ & $\cdot 8$ & $2 \cdot 98$ & $\cdot 4$ & $3 \cdot 55$ & $60 \cdot 0$ & $4 \cdot 18$ & $\cdot 6$ & 4.88 \\
\hline$\cdot 2$ & $2 \cdot 43$ & $\cdot 9$ & $2 \cdot 99$ & $\cdot 5$ & $3 \cdot 56$ & $\cdot 1$ & $4 \cdot 19$ & $\cdot 7$ & 4.90 \\
\hline$\cdot \overline{3}$ & $2 \cdot 44$ & $51 \cdot 0$ & $3 \cdot 0$ & 6 & 3.57 & 2 & $4 \cdot 20$ & .8 & 4.92 \\
\hline$\cdot 4$ & $2 \cdot 45$ & $\cdot 1$ & $3 \cdot 0$ & .7 & 3.59 & $\cdot 3$ & $4 \cdot 21$ & .9 & 4.93 \\
\hline 5 & $2 \cdot 46$ & 2 & 3 . & $\cdot 8$ & 3. & $\cdot 4$ & $4 \cdot 23$ & $65 \cdot 0$ & $4 \cdot 95$ \\
\hline$\cdot 6$ & $2 \cdot 47$ & $\cdot 3$ & $3 \cdot 04$ & $\cdot 9$ & $3 \cdot 61$ & $\cdot 5$ & $4 \cdot 24$ & $\cdot 1$ & $4 \cdot 97$ \\
\hline$\cdot 7$ & $2 \cdot 49$ & $\cdot 4$ & $3 \cdot 0$ & 56.0 & 3.63 & $\cdot 6$ & $4 \cdot 26$ & 2 & 4.98 \\
\hline 8 & 2.50 & .5 & 3. & $\cdot 1$ & $3 \cdot 64$ & $\cdot 7$ & $4 \cdot 27$ & $\cdot 3$ & 5.00 \\
\hline 9 & $2 \cdot 51$ & $\cdot 6$ & $3 \cdot 0$ & $\cdot 2$ & $3 \cdot 65$ & 8 & $4 \cdot 29$ & $\cdot 4$ & $5 \cdot 02$ \\
\hline $47 \cdot 0$ & $2 \cdot 52$ & 7 & 3 & $\cdot \overline{3}$ & $3 \cdot 67$ & $\cdot 9$ & $4 \cdot 30$ & $\cdot 5$ & $5 \cdot 04$ \\
\hline$\cdot 1$ & $2 \cdot 54$ & 8 & 3. & $\cdot 4$ & $3 \cdot 68$ & $61 \cdot 0$ & $4 \cdot 32$ & .6 & 5.05 \\
\hline$\cdot 2$ & $2 \cdot \overline{5}$ & 9 & $3 \cdot 1$ & .5 & $3 \cdot 69$ & $\cdot 1$ & $4 \cdot 33$ & $\cdot 7$ & $5 \cdot 07$ \\
\hline 3 & 2.56 & $52 \cdot 0$ & $3 \cdot 1$ & $\cdot 6$ & $3 \cdot 71$ & $\cdot 2$ & $4 \cdot 35$ & $\cdot 8$ & $5 \cdot 09$ \\
\hline$\cdot 4$ & $2 \cdot 57$ & $\cdot 1$ & $3 \cdot 14$ & .7 & $3 \cdot 72$ & $\cdot 3$ & $4 \cdot 36$ & .9 & $5 \cdot 11$ \\
\hline .5 & $\begin{array}{l}2.58 \\
2.68\end{array}$ & '2 & $3 \cdot 15$ & 8 & $3 \cdot 73$ & $\cdot 4$ & $4 \cdot 37$ & $66^{\circ} 0$ & $5 \cdot 12$ \\
\hline
\end{tabular}


Tabie IIb.-For Estimating the Percentage of Fat in Milk from the Specific Gravity of the Ether-fat Solution at $175^{\circ} \mathrm{C}$, ACCORdiNg to Soxhlet.

(b) Separated Milk.

\begin{tabular}{|c|c|c|c|c|c|c|c|c|c|}
\hline $\begin{array}{l}\text { Specific } \\
\text { gravity. }\end{array}$ & $\underset{\%}{\text { Fat }}$ & $\begin{array}{l}\text { Specific } \\
\text { gravity. }\end{array}$ & $\begin{array}{c}\text { Fat } \\
\%\end{array}$ & $\begin{array}{l}\text { Specific } \\
\text { gravity. }\end{array}$ & $\begin{array}{c}\text { Fat } \\
\%\end{array}$ & $\begin{array}{l}\text { Specific } \\
\text { gravity. }\end{array}$ & $\begin{array}{c}\text { Fat } \\
\%\end{array}$ & $\begin{array}{l}\text { Specific } \\
\text { gravity. }\end{array}$ & $\underset{\%}{\text { Fat }}$ \\
\hline $21 \cdot 1$ & 0.00 & $25 \cdot 5$ & 0.41 & $29 \cdot 9$ & 0.82 & $34 \cdot 3$ & 1.22 & $38 \cdot 7$ & $1 \cdot 64$ \\
\hline$\cdot 2$ & 0.01 & $\cdot 6$ & 0.42 & $30^{\circ} 0$ & 0.83 & $\cdot 4$ & $1 \cdot 23$ & $\cdot 8$ & 1.65 \\
\hline 3 & 0.02 & $\cdot 7$ & 0.43 & $\cdot 1$ & 0.84 & 5 & 1.24 & $\cdot 9$ & $1 \cdot 66$ \\
\hline 4 & 03 & 8 & 0.44 & $\cdot 2$ & 0.85 & $\cdot 6$ & $1 \cdot 24$ & 39.0 & $1 \cdot 67$ \\
\hline$\cdot 5$ & 04 & 9 & 0.45 & $\cdot 3$ & 0.86 & 7 & $1 \cdot 25$ & $\cdot 1$ & $1 \cdot 68$ \\
\hline$\cdot 6$ & 0.05 & $26 \cdot 0$ & 0.46 & $\cdot 4$ & 0.87 & 8 & $1 \cdot 26$ & $\cdot 2$ & $1 \cdot 69$ \\
\hline$\cdot 7$ & 0.06 & $\cdot 1$ & 0.47 & 5 & 0.88 & $\cdot 9$ & $1 \cdot 27$ & $\cdot 3$ & $1 \cdot 70$ \\
\hline 8 & 0.07 & 2 & 0.48 & $\cdot 6$ & 0.88 & $35 \cdot 0$ & $1 \cdot 28$ & $\cdot 4$ & 1.71 \\
\hline$\cdot 9$ & 0.08 & $\cdot 3$ & 0.49 & $\cdot 7$ & 0.89 & $\cdot 1$ & $1 \cdot 29$ & $\cdot 5$ & 1.72 \\
\hline $22 \cdot 0$ & 0.09 & $\cdot 4$ & 0.50 & 8 & 0.90 & 2 & $1 \cdot 30$ & $\cdot 6$ & 1.73 \\
\hline$\cdot 1$ & $0 \cdot 10$ & 5 & 0.50 & $\cdot 9$ & 0.91 & 3 & $1 \cdot 31$ & $\cdot 7$ & $1 \cdot 74$ \\
\hline 2 & $0 \cdot 11$ & 6 & 0.51 & $31 \cdot 0$ & 0.92 & $\cdot 4$ & $1 \cdot 32$ & 8 & $1 \cdot 75$ \\
\hline$\cdot 3$ & 0.12 & .7 & 0.52 & 1 & 0.93 & $\cdot \overline{5}$ & $1 \cdot 33$ & $\cdot 9$ & 1.76 \\
\hline$\cdot 4$ & $0 \cdot 13$ & 8 & 0.53 & $\cdot 2$ & 0.94 & 6 & $1 \cdot 33$ & $40 \cdot 0$ & $1 \cdot 77$ \\
\hline 5 & $0 \cdot 14$ & $\cdot 9$ & 0.54 & $\cdot 3$ & 0.95 & $\cdot 7$ & $1 \cdot 34$ & $\cdot 1$ & $1 \cdot 78$ \\
\hline$\cdot 6$ & 0.15 & $27 \cdot 0$ & 0.55 & $\cdot 4$ & 0.95 & $\cdot 8$ & $1 \cdot 35$ & $\cdot 2$ & 1.79 \\
\hline$\cdot 7$ & $0 \cdot 16$ & $\cdot 1$ & 0.56 & 5 & 0.96 & $\cdot 9$ & $1 \cdot 36$ & 3 & $1 ' 80$ \\
\hline 8 & 0.17 & $\cdot 2$ & 0.57 & 6 & 0.97 & $36 \cdot 0$ & $1 \cdot 37$ & $\cdot 4$ & 1.81 \\
\hline$\cdot 9$ & $0 \cdot 18$ & $\cdot 3$ & 0.58 & $\cdot 7$ & 0.98 & $\cdot 1$ & $1 \cdot 38$ & 5 & 1.82 \\
\hline $23 \cdot 0$ & $0 \cdot 19$ & $\cdot 4$ & 0.59 & $\cdot 8$ & 0.99 & $\cdot 2$ & $1 \cdot 39$ & 6 & $1 \cdot 83$ \\
\hline$\cdot 1$ & $0 \cdot 20$ & $\cdot 5$ & 0.60 & $\cdot 9$ & $1 \cdot 00$ & 3 & 1.40 & $\cdot 7$ & 1.84 \\
\hline$\cdot 2$ & $0 \cdot 21$ & 6 & 0.60 & $32 \cdot 0$ & 1.01 & $\cdot 4$ & $1 \cdot 41$ & 8 & 1.85 \\
\hline$\cdot 3$ & $0 \cdot 22$ & $\cdot 7$ & $0 \cdot 61$ & $\cdot 1$ & 1 & $\cdot \overline{5}$ & 1.42 & $\cdot 9$ & 1.86 \\
\hline$\cdot 4$ & 0.23 & $\cdot 8$ & 0.62 & $=2$ & 1 & $\cdot 6$ & 1.43 & $41 \cdot 0$ & $1 \cdot 87$ \\
\hline$\cdot 5$ & $0 \cdot 24$ & $\cdot 9$ & 0.63 & $\cdot 3$ & $1 \cdot 04$ & $\cdot 7$ & $1 \cdot 44$ & $\cdot 1$ & 1.88 \\
\hline$\cdot 6$ & 0.25 & $28 \cdot 0$ & 0.6 & $\cdot 4$ & 1 & 8 & 1.45 & 2 & 1.89 \\
\hline$\cdot 7$ & 0.25 & $\cdot 1$ & $0 \cdot 65$ & $\cdot \bar{\jmath}$ & I & $\cdot 9$ & $1 \cdot 46$ & $\cdot 3$ & 1.90 \\
\hline 8 & 0.26 & 2 & $0 \cdot 66$ & $\cdot 6$ & 1.06 & $37 \cdot 0$ & 1.47 & 4 & 1.91 \\
\hline .9 & 0.27 & 3 & 0. & $\cdot 7$ & 1 & $\cdot 1$ & 1 & $\cdot 5$ & 1.92 \\
\hline $24^{\circ} \cdot 0$ & $0 \cdot 28$ & $\cdot 4$ & 0.68 & 8 & 08 & $\cdot 2$ & 1.49 & 6 & 1.93 \\
\hline$\cdot 1$ & $0 \cdot 29$ & $\check{\tilde{J}}$ & 0.69 & $\cdot 9$ & & $\cdot 3$ & 1.50 & 7 & 1.94 \\
\hline$\cdot 2$ & $0 \cdot 30$ & $\cdot 6$ & 0.70 & $33 \cdot 0$ & 1 & $\cdot 4$ & 1.51 & 8 & 1.95 \\
\hline 3 & $0 \cdot 30$ & $\cdot 7$ & 0.71 & $\cdot 1$ & $1 \cdot 11$ & 5 & $1 \cdot 52$ & $\cdot 9$ & 1.96 \\
\hline$\cdot 4$ & 0.31 & $\cdot 8$ & 0.72 & 2 & $1 \cdot 12$ & 6 & 1.53 & $42 \cdot 0$ & 1.97 \\
\hline 5 & $0 \cdot 32$ & $\cdot 9$ & 0.73 & $\cdot 3$ & $1 \cdot 13$ & $\cdot 7$ & 1.54 & $\cdot 1$ & 1.98 \\
\hline 6 & $0: 33$ & $29 \cdot 0$ & 0.74 & $\cdot 4$ & $1 \cdot 14$ & 8 & 1.55 & $\cdot 2$ & 1.99 \\
\hline$\cdot 7$ & 0.34 & $\cdot 1$ & 0.75 & 5 & & 9 & 1.56 & $\cdot 3$ & 2.00 \\
\hline .8 & 0.35 & $\cdot 2$ & 0.76 & $\cdot 6$ & $1 \cdot 15$ & $38 \cdot 0$ & 1.57 & $\cdot 4$ & $2 \cdot 01$ \\
\hline$\cdot 9$ & $0 \cdot 36$ & $\cdot 3$ & 0.77 & $\cdot 7$ & $1 \cdot 16$ & $\cdot 1$ & 1.58 & $\cdot 5$ & $2 \cdot 02$ \\
\hline $25 \cdot 0$ & 0. & $\cdot 4$ & 0.78 & 8 & & $\cdot 2$ & $1 \cdot 59$ & $\cdot 6$ & $2 \cdot 03$ \\
\hline$\cdot 1$ & 0.38 & $\cdot 5$ & 0.79 & $\cdot 9$ & $1 \cdot 18$ & 3 & $1 \cdot 60$ & $\cdot 7$ & $2 \cdot 04$ \\
\hline$\cdot 2$ & $0 \cdot 39$ & $\cdot 6$ & 0.80 & $34^{\circ} 0$ & $1 \cdot 19$ & $\cdot 4$ & $1 \cdot 61$ & $\cdot 8$ & $2 \cdot 05$ \\
\hline 3 & 0 & $\cdot 7$ & 0.80 & $\cdot 1$ & $1 \cdot 20$ & 5 & $1 \cdot 62$ & $\cdot 9$ & 2.06 \\
\hline-4 & 0.40 & 8 & 0.81 & $\cdot 2$ & 1.21 & 6 & 1.63 & $43 \cdot 0$ & $2 \cdot 07$ \\
\hline
\end{tabular}




\section{MILK AND DAIRY PRODUCTS}

Table IIIa.-For Converting the Scale Divisions of the Zeiss Milk Fat Refractometer into Percentages of Fat, according to Dr. Naumann, Hali.e.

\begin{tabular}{|c|c|c|c|c|c|c|c|c|c|}
\hline $\begin{array}{c}\text { Scale } \\
\text { division. }\end{array}$ & $\begin{array}{c}\text { Fat } \\
\%\end{array}$ & $\begin{array}{c}\text { Scale } \\
\text { division. }\end{array}$ & $\underset{\%}{\text { Fat }}$ & $\begin{array}{c}\text { Scale } \\
\text { division. }\end{array}$ & $\begin{array}{c}\text { Fat } \\
\%\end{array}$ & $\begin{array}{c}\text { Scale } \\
\text { division. }\end{array}$ & $\underset{\%}{\text { Fat }}$ & $\begin{array}{c}\text { Scale } \\
\text { division. }\end{array}$ & $\begin{array}{c}\text { Fat } \\
\%\end{array}$ \\
\hline $20 \cdot 0$ & - & $24 \cdot 6$ & 0.42 & $29 \cdot 2$ & 0.89 & $33 \cdot 8$ & $1 \cdot 37$ & $38 \cdot 4$ & $1 \cdot 90$ \\
\hline$\cdot 1$ & - & $\cdot 7$ & 0.43 & 3 & 0.90 & .9 & $1 \cdot 38$ & .5 & 1.91 \\
\hline$\cdot 2$ & - & $\cdot 8$ & 0.44 & .4 & 0.91 & $34 \cdot 0$ & $1 \cdot 39$ & .6 & $\begin{array}{l}1.92 \\
\end{array}$ \\
\hline$\cdot 3$ & - & $\cdot 9$ & 0.45 & 5 & 0.92 & $\cdot 1$ & $1 \cdot 40$ & $\cdot 7$ & 1.93 \\
\hline$\cdot 4$ & - & $25 \cdot 0$ & 0.46 & $\cdot 6$ & 0.93 & $\cdot 2$ & 1.42 & 8 & 1.94 \\
\hline .5 & - & $\cdot 1$ & 0.47 & $\cdot 7$ & 0.94 & .3 & $1 \cdot 48$ & .9 & $\begin{array}{l}1.95 \\
\end{array}$ \\
\hline$\cdot 6$ & 0.00 & 2 & 0.48 & .8 & 0.95 & .4 & $1 \cdot 44$ & 39.0 & 1.96 \\
\hline$\cdot 7$ & 0.01 & $\cdot 3$ & 0.49 & $\cdot 9$ & 0.96 & $\cdot 5$ & $1 \cdot 45$ & $\cdot 1$ & 1.98 \\
\hline .8 & 0.02 & $\cdot 4$ & 0.50 & $30^{\circ} 0$ & 0.97 & .6 & $1 \cdot 46$ & 2 & 1.99 \\
\hline .9 & 0.03 & .5 & 0.51 & $\cdot 1$ & 0.98 & .7 & $1 \cdot 47$ & .3 & 2.00 \\
\hline $21 \cdot 0$ & 0.04 & $\cdot 6$ & 0.52 & $\cdot 2$ & 0.99 & $\cdot 8$ & $1 \cdot 48$ & $\cdot 4$ & $2 \cdot 02$ \\
\hline$\cdot 1$ & 0.05 & .7 & 0.53 & $\cdot 3$ & $1 \cdot 00$ & .9 & $1 \cdot 49$ & $\cdot 5$ & $2 \cdot 03$ \\
\hline$\cdot 2$ & 0.06 & 8 & 0.54 & $\cdot 4$ & 1.01 & $35 \cdot 0$ & $1 \cdot 50$ & .6 & 2.04 \\
\hline$\cdot 3$ & 0.08 & .9 & 0.55 & .5 & $1 \cdot 02$ & $\cdot 1$ & 1.51 & .7 & 2.05 \\
\hline$\cdot 4$ & 0.09 & $26 \cdot 0$ & 0.57 & 6 & $1 \cdot 03$ & $\cdot 2$ & $1 \cdot 52$ & $\cdot 8$ & $2 \cdot 07$ \\
\hline 5 & $0 \cdot 10$ & $\cdot 1$ & 0.58 & $\cdot 7$ & $1 \cdot 04$ & 3 & 1.54 & $\cdot 9$ & 2.08 \\
\hline 6 & 0.11 & .2 & 0.59 & .8 & 1.05 & .4 & $1 \cdot 55$ & $40^{\circ} 0$ & 2.09 \\
\hline$\cdot 7$ & $0 \cdot 12$ & $\cdot 3$ & 0.60 & $\cdot 9$ & $1 \cdot 06$ & $\cdot 5$ & $1 \cdot 56$ & $\cdot 1$ & $2 \cdot 10$ \\
\hline .8 & $0 \cdot 13$ & $\cdot 4$ & $0 \cdot 61$ & $31 \cdot 0$ & 1.07 & $\cdot 6$ & 1.57 & $\cdot 2$ & $2 \cdot 12$ \\
\hline .9 & $0 \cdot 14$ & .5 & 0.62 & $\cdot 1$ & 1.08 & $\cdot 7$ & 1.58 & .3 & $2 \cdot 13$ \\
\hline $22 \cdot 0$ & $0 \cdot 15$ & .6 & 0.63 & .2 & 1.09 & .8 & 1.59 & .4 & $2 \cdot 14$ \\
\hline$\cdot 1$ & $0 \cdot 16$ & $\cdot 7$ & 0.64 & $\cdot 3$ & $1 \cdot 10$ & $\cdot 9$ & $1 \cdot 60$ & $\cdot 5$ & $2 \cdot 15$ \\
\hline .2 & 0.17 & .8 & 0.65 & 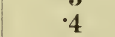 & $1 \cdot 11$ & $36 \cdot 0$ & $1 \cdot 61$ & .6 & $2 \cdot 16$ \\
\hline 3 & $0 \cdot 18$ & .9 & 0.66 & .5 & $1 \cdot 12$ & $\cdot 1$ & 1.62 & $\cdot 7$ & $2 \cdot 18$ \\
\hline$\cdot 4$ & $0 \cdot 19$ & $27 \cdot 0$ & 0.67 & $\cdot 6$ & $1 \cdot 13$ & $\cdot 2$ & $1 \cdot 64$ & 8 & $2 \cdot 20$ \\
\hline$\cdot 5$ & $0 \cdot 20$ & $\cdot 1$ & $0 \cdot 68$ & 7 & $1 \cdot 14$ & $\cdot 3$ & $1 \cdot 65$ & .9 & $2 \cdot 21$ \\
\hline 6 & 0.21 & 2 & 0.69 & 8 & $1 \cdot 15$ & $\cdot 4$ & $1 \cdot 66$ & $41^{\circ} 0$ & $2 \cdot 23$ \\
\hline .7 & 0.22 & $\cdot 3$ & 0.70 & $\cdot 9$ & $1 \cdot 16$ & .5 & $\mathrm{I} \cdot 67$ & $\cdot 1$ & $2 \cdot 24$ \\
\hline .8 & $0 \cdot 23$ & $\cdot 4$ & 0.71 & $32 \cdot 0$ & $1 \cdot 17$ & $\cdot 6$ & $1 \cdot 68$ & 2 & $2 \cdot 25$ \\
\hline$\cdot 9$ & 0.24 & $\cdot 5$ & 0.72 & $\cdot 1$ & $1 \cdot 18$ & $\cdot 7$ & $1 \cdot 69$ & $\cdot 3$ & $2 \cdot 26$ \\
\hline $23 \cdot 0$ & $0 \cdot 25$ & $\cdot 6$ & 0.73 & 2 & $1 \cdot 19$ & .8 & 1.70 & $\cdot 4$ & $2 \cdot 27$ \\
\hline$\cdot 1$ & $0 \cdot 26$ & $\cdot 7$ & 0.74 & $\cdot 3$ & $1 \cdot 20$ & $\cdot 9$ & 1.71 & .5 & $2 \cdot 28$ \\
\hline$\cdot 2$ & $0 \cdot 27$ & $\cdot 8$ & 0.75 & $\cdot 4$ & $1 \cdot 22$ & $37 \cdot 0$ & 1.72 & $\cdot 6$ & $2 \cdot 30$ \\
\hline$\cdot 3$ & 0.28 & $\cdot 9$ & 0.76 & $\cdot 5$ & 1.23 & $\cdot 1$ & 1.73 & $\cdot 7$ & $2 \cdot 32$ \\
\hline$\cdot 4$ & $0 \cdot 29$ & $28 \cdot 0$ & 077 & 6 & $1 \cdot 24$ & $\cdot 2$ & $1 \cdot 75$ & 8 & $2 \cdot 33$ \\
\hline$\cdot 5$ & $0 \cdot 30$ & $\cdot 1$ & 0.78 & $\cdot 7$ & $1 \cdot 25$ & $\cdot 3$ & $1 \cdot 76$ & $\cdot 9$ & $2 \cdot 34$ \\
\hline$\cdot 6$ & 0.31 & 2 & 0.79 & $\cdot 8$ & $1 \cdot 26$ & $\cdot 4$ & 178 & $42 \cdot 0$ & $2 \cdot 35$ \\
\hline$\cdot 7$ & $0 \cdot 32$ & $\cdot 3$ & 0.80 & $\cdot 9$ & $1 \cdot 27$ & $\cdot 5$ & $1 \cdot 79$ & $\cdot 1$ & $2 \cdot 37$ \\
\hline .8 & $0 \cdot 33$ & $\cdot 4$ & 0.81 & $33 \cdot 0$ & $1 \cdot 28$ & 6 & 1.80 & 2 & $2 \cdot 38$ \\
\hline 9 & $0 \cdot 34$ & $\cdot 5$ & $0 \cdot 82$ & $\cdot 1$ & $1 \cdot 29$ & $\cdot 7$ & $1 \cdot 81$ & $\cdot 3$ & $2 \cdot 39$ \\
\hline $24^{\circ} 0$ & $0 \cdot 36$ & $\cdot 6$ & 0.83 & $\cdot 2$ & $1 \cdot 30$ & 8 & $1 \cdot 82$ & $\cdot 4$ & $2 \cdot 40$ \\
\hline$\cdot 1$ & 0.37 & $\cdot 7$ & 0.84 & $\cdot 3$ & $1 \cdot 31$ & $\cdot 9$ & $1 \cdot 84$ & .5 & $2 \cdot 41$ \\
\hline$\cdot 2$ & 0.38 & 8 & 0.85 & $\cdot 4$ & $1 \cdot 32$ & $38 \cdot 0$ & $1 \cdot 85$ & 6 & $2 \cdot 43$ \\
\hline .3 & $0 \cdot 39$ & .9 & 0.86 & $\cdot 5$ & 1.34 & $\cdot 1$ & 1.87 & .7 & $2 \cdot 44$ \\
\hline$\cdot 4$ & 0.40 & $29 \cdot 0$ & 0.87 & $\cdot 6$ & $1 \cdot 35$ & 2 & 1.88 & .8 & $2 \cdot 46$ \\
\hline •5 & 0.41 & 1 & 0.88 & $\cdot 7$ & $1 \cdot 36$ & $\cdot 3$ & $1 \cdot 89$ & $\cdot 9$ & $2 \cdot 47$ \\
\hline
\end{tabular}




\section{APPENDIX}

Table IIIb. - For Convertist: the Scale Divisions of the Zeiss Milk fat Refractometer into Percentages of Fat, according to Dr. Naumans, Halle.

\begin{tabular}{|c|c|c|c|c|c|c|c|c|c|}
\hline $\begin{array}{c}\text { Scale } \\
\text { division. }\end{array}$ & $\begin{array}{c}\text { Fat } \\
\%\end{array}$ & $\begin{array}{c}\text { Scale } \\
\text { division. }\end{array}$ & $\begin{array}{c}\text { Fat } \\
\%\end{array}$ & $\begin{array}{c}\text { Scale } \\
\text { division. }\end{array}$ & $\begin{array}{c}\text { Fat } \\
\%\end{array}$ & $\begin{array}{c}\text { Scale } \\
\text { division. }\end{array}$ & $\begin{array}{c}\text { Fat } \\
\%\end{array}$ & $\begin{array}{c}\text { Scale } \\
\text { division. }\end{array}$ & $\begin{array}{c}\text { Fat } \\
\%\end{array}$ \\
\hline $43^{\circ} 0$ & $2 \cdot 49$ & $47 \cdot 7$ & $3 \cdot 16$ & $52 \cdot 3$ & $3 \cdot 89$ & $56 \cdot 9$ & $4 \cdot 67$ & $61 \cdot 5$ & 5.54 \\
\hline$\cdot 1$ & 2.50 & 8 & $3 \cdot 17$ & 4 & $3 \cdot 90$ & $57 \cdot 0$ & $4 \cdot 69$ & 6 & $5 \cdot 56$ \\
\hline$\cdot 2$ & $2 \cdot 51$ & .9 & $3 \cdot 18$ & $\cdot 5$ & 3.92 & $\cdot 1$ & 4.71 & $\cdot 7$ & $5 \cdot 58$ \\
\hline 3 & $2 \cdot 52$ & $48^{\circ} 0$ & $3 \cdot 20$ & 6 & 3.93 & -2 & $4 \cdot 73$ & $\cdot 8$ & $5 \cdot 60$ \\
\hline .4 & $2 \cdot 54$ & 1 & 3.21 & 7 & 3.95 & 3 & 4.75 & $\cdot 9$ & $5 \cdot 61$ \\
\hline$\cdot 5$ & $2 \cdot 55$ & 2 & $3 \cdot 23$ & 8 & 3.97 & $\cdot 4$ & $4 \cdot 76$ & $62 \cdot 0$ & $5 \cdot 63$ \\
\hline .6 & $2 \cdot 56$ & 3 & $3: 25$ & $\cdot 9$ & 3.99 & 5 & 4.78 & $\cdot 1$ & 5.65 \\
\hline 7 & $2 \cdot 58$ & $\cdot 4$ & $3 \cdot 27$ & $53 \cdot 0$ & $4 \cdot 01$ & 6 & $4 \cdot 80$ & $\cdot 2$ & $5 \cdot 66$ \\
\hline$\cdot 8$ & $2 \cdot 60$ & 5 & $3 \cdot 28$ & $\cdot 1$ & $4 \cdot 03$ & $\cdot 7$ & $4 \cdot 82$ & 3 & $5 \cdot 68$ \\
\hline .9 & $2 \cdot 61$ & $\cdot 6$ & $3 \cdot 30$ & 2 & $4 \cdot() 4$ & 8 & $4 \cdot 84$ & $\cdot 4$ & $5 \cdot 70$ \\
\hline $44 \cdot 0$ & $2 \cdot 63$ & $\cdot 7$ & $3 \cdot 32$ & $\cdot 3$ & $4 \cdot 06$ & .9 & 4.86 & $\cdot \overline{5}$ & 5.72 \\
\hline$\cdot 1$ & $2 \cdot 64$ & .8 & $3 \cdot 33$ & 4 & 4.07 & $58 \cdot 0$ & $4 \cdot 88$ & 6 & 5.74 \\
\hline 2 & $2 \cdot 65$ & 9 & $3 \cdot 34$ & $\cdot 5$ & 4.09 & $\cdot 1$ & $4 \cdot 90$ & $\cdot 7$ & $5 \cdot 76$ \\
\hline .3 & $2 \cdot 67$ & $49 \cdot 0$ & $3 \cdot 36$ & $\cdot 6$ & $4 \cdot 10$ & $\cdot 2$ & $4 \cdot 92$ & 8 & $5 \cdot 78$ \\
\hline$\cdot 4$ & $2 \cdot 68$ & $\cdot 1$ & $3 \cdot 38$ & $\cdot 7$ & $4 \cdot 12$ & $\cdot 3$ & $4 \cdot 94$ & $\cdot 9$ & $5 \cdot 80$ \\
\hline 5 & $2 \cdot 70$ & $\cdot 2$ & $3 \cdot 40$ & 8 & $4 \cdot 14$ & $\cdot 4$ & $4 \cdot 95$ & $63^{\circ} 0$ & $5 \cdot 82$ \\
\hline$\cdot 6$ & $2 \cdot 71$ & ·3 & $3 \cdot 42$ & $\cdot 9$ & $4 \cdot 16$ & 5 & 4.97 & $\cdot 1$ & $5 \cdot 84$ \\
\hline$\cdot 7$ & $2 \cdot 72$ & $\cdot 4$ & $3 \cdot 43$ & $54 \cdot 0$ & $4 \cdot 18$ & 6 & 4.98 & 2 & 5.86 \\
\hline 8 & $2 \cdot 74$ & .5 & $3 \cdot 44$ & $\cdot 1$ & $4 \cdot 20$ & $\cdot 7$ & $5 \cdot 00$ & $\cdot 3$ & 5.88 \\
\hline$\cdot 9$ & $2 \cdot 7 \tilde{5}$ & $\cdot 6$ & $3 \cdot 45$ & $\cdot 2$ & $4 \cdot 22$ & $\cdot 8$ & $5 \cdot 02$ & $\cdot 4$ & $5 \cdot 90$ \\
\hline $45 \cdot 0$ & $2 \cdot 77$ & 7 & $3 \cdot 46$ & $\cdot 3$ & $4 \cdot 23$ & $\cdot 9$ & $5 \cdot 04$ & .5 & 5.92 \\
\hline$\cdot 1$ & $2 \cdot 78$ & .8 & $3 \cdot 48$ & .4 & 4.25 & $59 \cdot 0$ & $5 \cdot 06$ & 6 & 5.94 \\
\hline$\cdot 2$ & $2 \cdot 79$ & $\cdot 9$ & $3 \cdot 50$ & 5 & $4 \cdot 26$ & $\cdot 1$ & $5 \cdot 08$ & $\cdot 7$ & 5.96 \\
\hline 3 & $2 \cdot 80$ & $50^{\circ} 0$ & $3 \cdot 51$ & 6 & $4 \cdot 28$ & $\cdot 2$ & $5 \cdot 10$ & 8 & 5.98 \\
\hline 4 & $2 \cdot 82$ & $\cdot 1$ & $3 \cdot 53$ & 7 & $4 \cdot 29$ & $\cdot 3$ & $5 \cdot 11$ & $\cdot 9$ & 6.00 \\
\hline 5 & $2 \cdot 84$ & 2 & 3.55 & .8 & $4 \cdot 31$ & $\cdot 4$ & $5 \cdot 13$ & $64 \cdot 0$ & 6.02 \\
\hline .6 & $2 \cdot 85$ & $\cdot 3$ & 356 & $\cdot 9$ & $4 \cdot 33$ & .5 & $5 \cdot 15$ & $\cdot 1$ & 6.04 \\
\hline$\cdot 7$ & $2 \cdot 87$ & $\cdot 4$ & 3.57 & $55^{\circ} 0$ & $4 \cdot 35$ & $\cdot 6$ & $5 \cdot 17$ & $\cdot 2$ & 6.07 \\
\hline 8 & $2 \cdot 88$ & $\cdot 5$ & $3 \cdot 59$ & · & $4 \cdot 37$ & $\cdot 7$ & $5 \cdot 19$ & $\cdot 3$ & 6.09 \\
\hline 9 & $2 \cdot 89$ & $\cdot 6$ & $3 \cdot 60$ & $\cdot 2$ & $4 \cdot 38$ & 8 & $5 \cdot 20$ & $\cdot 4$ & $6 \cdot 12$ \\
\hline $46 \cdot 0$ & $2 \cdot 90$ & $\cdot 7$ & $3 \cdot 61$ & 3 & $4 \cdot 40$ & $\cdot 9$ & $5 \cdot 22$ & $\cdot 5$ & $6 \cdot 14$ \\
\hline$\cdot 1$ & $2 \cdot 92$ & 8 & $3 \cdot 63$ & $\cdot 4$ & $4 \cdot 42$ & $60 \cdot 0$ & $5 \cdot 24$ & $\cdot 6$ & $6 \cdot 16$ \\
\hline 2 & $2 \cdot 93$ & 9 & $3 \cdot 64$ & $\cdot 5$ & 4.43 & $\cdot 1$ & $5 \cdot 26$ & $\cdot 7$ & $6 \cdot 18$ \\
\hline$\cdot 3$ & $2 \cdot 94$ & $510^{\circ}$ & $3 \cdot 66$ & $\cdot 6$ & $4 \cdot 44$ & 2 & $5 \cdot 28$ & .8 & $6 \cdot 20$ \\
\hline$\cdot 4$ & $2 \cdot 96$ & $\cdot 1$ & $3 \cdot 67$ & $\cdot 7$ & $4 \cdot 46$ & $\cdot 3$ & $5 \cdot 30$ & .9 & $6 \cdot 22$ \\
\hline$\cdot 5$ & $2 \cdot 98$ & $\cdot 2$ & $3 \cdot 68$ & 8 & $4 \cdot 48$ & $\cdot 4$ & $5 \cdot 32$ & $65 \cdot 0$ & $6 \cdot 24$ \\
\hline 6 & $3 \cdot 00$ & 3 & $3 \cdot 70$ & $\cdot 9$ & $4 \cdot 49$ & $\cdot 5$ & $5 \cdot 34$ & $\cdot 1$ & $6 \cdot 27$ \\
\hline 7 & 3.01 & $\cdot 4$ & $3 \cdot 72$ & $56 \cdot 0$ & $4 \cdot 51$ & $\cdot 6$ & $5 \cdot 36$ & 2 & 6.29 \\
\hline 8 & $3 \cdot 02$ & $\cdot 5$ & 3.74 & $\cdot 1$ & $4 \cdot 53$ & $\cdot 7$ & $5 \cdot 38$ & 3 & $6 \cdot 31$ \\
\hline 9 & 3.03 & $\cdot 6$ & $3 \cdot 76$ & $\cdot 2$ & $4 \cdot 55$ & 8 & $5 \cdot 40$ & $\cdot 4$ & 6.34 \\
\hline $47 \cdot 0$ & $3 \cdot 05$ & 7 & $3 \cdot 78$ & $\cdot 3$ & $4 \cdot 57$ & .9 & $5 \cdot 42$ & $\cdot 5$ & $6 \cdot 36$ \\
\hline$\cdot 1$ & 3.06 & 8 & $3 \cdot 80$ & $\cdot 4$ & $4 \cdot 59$ & $61^{\circ} 0$ & $5 \cdot 44$ & 6 & $6 \cdot 38$ \\
\hline 2 & 3.08 & $\cdot 9$ & $3 \cdot 82$ & 5 & $4 \cdot 60$ & $\cdot 1$ & $5 \cdot 46$ & 7 & $6 \cdot 40$ \\
\hline$\cdot 3$ & $3 \cdot 10$ & $52 \cdot 0$ & $3 \cdot 84$ & $\cdot 6$ & $4 \cdot 61$ & 2 & $5 \cdot 48$ & 8 & 6.42 \\
\hline$\cdot 4$ & $3 \cdot 12$ & $\cdot 1$ & $3 \cdot 85$ & $\cdot 7$ & $4 \cdot 63$ & $\cdot 3$ & $5 \cdot 50$ & $\cdot 9$ & $6 \cdot 44$ \\
\hline 5 & $3 \cdot 14$ & $\cdot 2$ & $3 \cdot 87$ & .8 & $4 \cdot 65$ & $\cdot 4$ & $5 \cdot 52$ & 66.0 & $6 \cdot 46$ \\
\hline • 6 & $3 \cdot 15$ & & & & & & & & \\
\hline
\end{tabular}




\section{MILK AND DAIRY PRODUCTS}

Table IV.--For Determining the Amount of Fat in Cream from the Percentage of Total Solids, according to M. Weibuli.

\begin{tabular}{|c|c|c|c|c|c|c|c|c|c|}
\hline $\begin{array}{c}\text { Total } \\
\text { Solids. } \\
\%\end{array}$ & $\begin{array}{c}\text { Fat } \\
\%\end{array}$ & $\begin{array}{c}\text { Total } \\
\text { Solids. } \\
\%\end{array}$ & $\begin{array}{c}\text { Fat } \\
\%\end{array}$ & $\begin{array}{l}\text { 'l'otal } \\
\text { Solids. } \\
\%\end{array}$ & $\begin{array}{c}\text { Fat } \\
\%\end{array}$ & $\begin{array}{c}\text { Total } \\
\text { Solids. } \\
\%\end{array}$ & $\begin{array}{c}\text { Fat } \\
\%\end{array}$ & $\begin{array}{c}\text { Total } \\
\text { Solids. } \\
\%\end{array}$ & $\begin{array}{c}\text { Fat } \\
\%\end{array}$ \\
\hline $17 \cdot 7$ & $10 \cdot 0$ & $22 \cdot 6$ & $15 \cdot 4$ & $27 \cdot 5$ & $20 \cdot 8$ & $32 \cdot 4$ & $26 \cdot 1$ & $37 \cdot 3$ & $31 \%$ \\
\hline $17 \cdot 8$ & $10 \cdot 1$ & $22 \cdot 7$ & $15 \cdot 5$ & $27 \cdot 6$ & $20 \cdot 9$ & $32 \cdot 5$ & $26 \cdot 3$ & $37 \cdot 4$ & $31 \cdot 6$ \\
\hline 17.9 & $10 \cdot 2$ & $22 \cdot 8$ & $15 \cdot 6$ & $27 \cdot 7$ & $21 \cdot 0$ & $32 \cdot 6$ & $26 \cdot 4$ & $37 \cdot 5$ & $31 \cdot 8$ \\
\hline $18 \cdot 0$ & $10 \cdot 3$ & $22 \cdot 9$ & $15 \cdot 7$ & $27 \cdot 8$ & $21 \cdot 1$ & $32 \cdot 7$ & 200 & $37 \cdot 6$ & $31 \cdot 9$ \\
\hline $18 \cdot 1$ & $10 \cdot 4$ & $23 \cdot 0$ & $15 \cdot 8$ & $27 \cdot 9$ & $21 \cdot 2$ & $32 \cdot 8$ & $26 \cdot 6$ & $37 \cdot 7$ & $32 \cdot 0$ \\
\hline $18 \cdot 2$ & 10.5 & $23 \cdot 1$ & $15 \cdot 9$ & $28 \cdot 0$ & $21 \cdot 3$ & $32 \cdot 9$ & $26 \cdot 7$ & $37 \cdot 8$ & $32 \cdot 1$ \\
\hline $18 \cdot 3$ & $10 \cdot 6$ & $23 \cdot 2$ & $16 \cdot 0$ & $28 \cdot 1$ & $21 \cdot 4$ & $33 \cdot 0$ & $26 \cdot 8$ & $37 \cdot 9$ & $32 \cdot 2$ \\
\hline $18 \cdot 4$ & $10 \cdot 7$ & $23 \cdot 3$ & $16 \cdot 1$ & $28 \cdot 2$ & $21 \cdot 5$ & $33 \cdot 1$ & $26 \cdot 9$ & $38 \cdot 0$ & $32 \cdot 3$ \\
\hline $18 \cdot 5$ & $10 \cdot 9$ & $23 \cdot 4$ & $16 \cdot 2$ & $28 \cdot 3$ & $21 \cdot 6$ & $33 \cdot 2$ & $27 \cdot 0$ & 38 & $32 \cdot 4$ \\
\hline $18 \cdot 6$ & $11 \cdot 0$ & $23 \cdot 5$ & $16 \cdot 4$ & $28 \cdot 4$ & $21 \cdot 7$ & $33 \cdot 3$ & $27 \cdot 1$ & $38 \cdot 2$ & $32 \cdot 5$ \\
\hline $18 \cdot 7$ & $11 \cdot 1$ & $23 \cdot 6$ & $16 \cdot 5$ & $28 \cdot 5$ & $21 \cdot 9$ & $33 \cdot 4$ & $27 \cdot 2$ & $38 \cdot 3$ & $32 \cdot 6$ \\
\hline $18 \cdot 8$ & $11 \cdot 2$ & $23 \cdot 7$ & $16 \cdot 6$ & $28 \cdot 6$ & $22 \cdot 0$ & 33 & $27 \cdot 4$ & 3 & $32 \cdot 7$ \\
\hline $18 \cdot 9$ & $11 \cdot 3$ & $23 \cdot 8$ & $16 \cdot 7$ & $28 \cdot 7$ & $22 \cdot 1$ & $33 \cdot 6$ & $27 \cdot 5$ & ? & $32 \cdot 9$ \\
\hline $19 \cdot 0$ & $11 \cdot 4$ & $23 \cdot 9$ & $16 \cdot 8$ & $28 \cdot 8$ & $22 \cdot 2$ & $33 \cdot 7$ & $27 \cdot 6$ & $38 \cdot 6$ & $33 \cdot 0$ \\
\hline $19 \cdot 1$ & $11 \cdot 5$ & $24 \cdot 0$ & $16 \cdot 9$ & $28 \cdot 9$ & $22 \cdot 3$ & 33 & $27 \cdot 7$ & 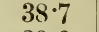 & $33 \cdot 1$ \\
\hline $19 \cdot 2$ & $11 \cdot 6$ & $24 \cdot 1$ & $17 \cdot 0$ & $29 \cdot 0$ & $22 \cdot 4$ & $33 \cdot 9$ & $27 \cdot 8$ & 8 & $33 \cdot 2$ \\
\hline $19 \cdot 3$ & $11 \cdot 7$ & $24 \cdot 2$ & $17 \cdot 1$ & $29 \cdot 1$ & $22 \cdot 5$ & 34 & $27^{\circ}$ & 0 & $33 \cdot 3$ \\
\hline $19 \cdot 4$ & $11 \cdot 8$ & $24 \cdot 3$ & $17 \cdot 2$ & $29 \cdot 2$ & $22 \cdot 6$ & 34 & 28 & 39 & $33 \cdot 4$ \\
\hline $19 \cdot 5$ & $12 \cdot 0$ & $24 \cdot 4$ & $17 \cdot 3$ & $29 \cdot 3$ & $22 \cdot 7$ & $34 \div 2$ & $28 \cdot 1$ & 3 & 33.5 \\
\hline $19 \cdot 6$ & $12 \cdot 1$ & $24 \cdot 5$ & $17 \cdot 5$ & $29 \cdot 4$ & $22 \cdot 8$ & $34 \cdot 3$ & $28 \cdot 2$ & 39 & $33 \cdot 6$ \\
\hline $19 \cdot 7$ & $12 \cdot 2$ & $24 \cdot 6$ & $17 \cdot 6$ & $29 \cdot 5$ & $23 \cdot 0$ & $34^{\circ}$ & $28:$ & $39 \cdot 3$ & $33 \cdot 7$ \\
\hline $19 \cdot 8$ & $12 \cdot 3$ & $24 \cdot 7$ & $17 \cdot 7$ & $29 \cdot 6$ & $23 \cdot 1$ & 34.5 & $28 \cdot 5$ & 39.4 & $33 \cdot 8$ \\
\hline $19 \cdot 9$ & $12 \cdot 4$ & $24 \cdot 8$ & $17 \cdot 8$ & $29 \cdot 7$ & $23 \cdot 2$ & 3 & 0 & & $34 \cdot 0$ \\
\hline $20 \cdot 0$ & $12 \cdot 5$ & $24 \cdot 9$ & $17 \cdot 9$ & $29 \cdot 8$ & $23 \cdot 3$ & 34 & $28 \cdot 7$ & 3 & $34 \cdot 1$ \\
\hline $20 \cdot 1$ & $12 \cdot 6$ & $25 \cdot 0$ & $18 \cdot 0$ & $29 \cdot 9$ & $23 \cdot 4$ & $34 \cdot 8$ & $28 \cdot 8$ & 7 & $34 \cdot 2$ \\
\hline $20 \cdot 2$ & $12 \cdot 7$ & $25 \cdot 1$ & $18 \cdot 1$ & $30^{\circ} 0$ & 5 & 3 & 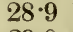 & 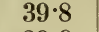 & $34 \cdot 3$ \\
\hline $20 \cdot 3$ & $12 \cdot 8$ & $25 \cdot 2$ & $18 \cdot 2$ & $30 \cdot 1$ & 6 & & 0.0 & & $34 \cdot 4$ \\
\hline 20 & $12 \cdot 9$ & $25 \cdot 3$ & $18 \cdot 3$ & $30 \cdot 2$ & $\cdot 7$ & 3 & 1 & 40 & $34 \cdot 5$ \\
\hline $20 \cdot 5$ & $13 \cdot 1$ & $25 \cdot 4$ & $18 \cdot 4$ & $30 \cdot 3$ & $23 \cdot 8$ & $35 \cdot 2$ & $29 \cdot 2$ & $40 \cdot 1$ & $34 \cdot 6$ \\
\hline $20 \cdot 6$ & $13 \cdot 2$ & $25 \cdot 5$ & $18 \cdot 6$ & 30.4 & $3 \cdot 9$ & $35 \cdot 3$ & $29 \cdot 3$ & & $34 \cdot 7$ \\
\hline 20 & $13 \cdot 3$ & $25 \cdot 6$ & 1 & $30 \cdot 5$ & 1 & 35 & $29^{\circ}$ & 40 & $34 \cdot 8$ \\
\hline 20 & $13 \cdot 4$ & $25 \cdot 7$ & & $30 \cdot 6$ & 2 & 35 & $29^{\circ}$ & 40 & $34 \cdot 9$ \\
\hline $20 \cdot 9$ & $13 \cdot 5$ & $25 \cdot 8$ & & $30 \cdot 7$ & $24 \cdot 3$ & & 20.7 & & $35 \cdot 1$ \\
\hline 21 & $13 \cdot 6$ & $5 \cdot 9$ & 19 & $30 \cdot 8$ & & ? & 29 & & $35 \cdot 2$ \\
\hline $21^{\circ}$ & $13 \cdot 7$ & $26 \cdot 0$ & & $30 \cdot 9$ & $24 \cdot 5$ & 35 & $29 \cdot 9$ & $40 \cdot 7$ & $35 \cdot 3$ \\
\hline $21 \cdot 2$ & $13 \cdot 8$ & $26 \cdot 1$ & & $31 \cdot 0$ & & & $30 \cdot 0$ & & $35 \cdot 4$ \\
\hline 2 & 9 & $26 \cdot 2$ & & $31 \cdot 1$ & & $\sigma$ & 3 & & $35 \cdot 5$ \\
\hline $21 \cdot 4$ & $14 \cdot 0$ & $26 \cdot 3$ & 19.4 & $31 \cdot 2$ & & 36 & 3 & $41 \cdot 0$ & $35 \cdot 6$ \\
\hline 21 . & $14 \cdot 2$ & $26 \cdot 4$ & & $31 \cdot 3$ & & $36 \cdot 2$ & & & $35 \cdot 7$ \\
\hline 21 & 1 & $26 \cdot 5$ & & 3 & & 36 & 30 & 41 & 35.8 \\
\hline $21 \cdot 7$ & $14 \cdot 4$ & $26 \cdot 6$ & & $31 \cdot 5$ & & & & 41 & $35 \cdot 9$ \\
\hline 21 & $14 \cdot 5$ & $26 \cdot 7$ & & $31 \cdot 6$ & & & & & $36 \cdot 0$ \\
\hline 21 & $14 \cdot 6$ & $26 \cdot 8$ & 20 & $31 \cdot 7$ & & 36 & & $41 \cdot 5$ & $36 \cdot 2$ \\
\hline $22 \cdot 0$ & $14 \cdot 7$ & $26 \cdot 9$ & 20 & $31 \cdot 8$ & & $36 \cdot 7$ & 30 & $41 \cdot 6$ & $36 \cdot 3$ \\
\hline 22 & & $27 \cdot 0$ & & $31 \cdot 9$ & & & & $41 \cdot 7$ & $36 \cdot 4$ \\
\hline $22 \cdot 2$ & $14 \cdot 9$ & $27 \cdot 1$ & $20 \cdot 3$ & $32 \cdot 0$ & $25 \cdot 7$ & $36 \cdot 9$ & $31 \cdot 1$ & $41 \cdot 8$ & $36 \cdot 5$ \\
\hline $22 \cdot 3$ & 15 & $27 \cdot 2$ & 20 & $32 \cdot 1$ & & $37 \cdot 0$ & $31 \cdot 2$ & $41 \cdot 9$ & $36 \cdot 6$ \\
\hline $22 \cdot 4$ & $15 \cdot 1$ & $27 \cdot 3$ & 20 & $32 \cdot 2$ & $25 \cdot 9$ & $37 \cdot 1$ & $31 \cdot 3$ & $42 \cdot 0$ & $36 \cdot 7$ \\
\hline $22 \cdot 5$ & $15 \cdot 3$ & $27 \cdot 4$ & $20 \cdot 6$ & $32 \cdot 3$ & $26^{\circ} 0$ & $37 \cdot 2$ & $31 \cdot 4$ & $42 \cdot 1$ & $36 \cdot 8$ \\
\hline
\end{tabular}


Table V.-For Determining the Amount of Milk Sugar from the Weitht of Copper, according to Soxhlet.

\begin{tabular}{|c|c|c|c|c|c|c|c|c|c|c|c|}
\hline 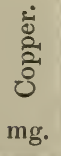 & 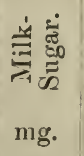 & 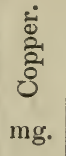 & 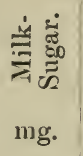 & 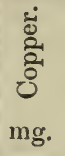 & 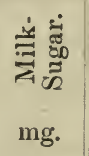 & 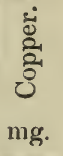 & 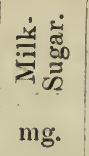 & $\begin{array}{l}\text { m.ँ } \\
\text { mg. } \\
\text { ठัँ }\end{array}$ & 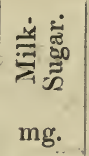 & 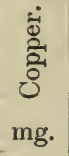 & 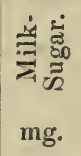 \\
\hline 140 & $101 \cdot 3$ & 184 & $134 \cdot 7$ & 228 & $167 \cdot 9$ & 272 & $201 \cdot 9$ & 316 & $236 \cdot 8$ & 360 & $272 \cdot 1$ \\
\hline 141 & $102 \cdot 1$ & 185 & $135 \cdot 4$ & 229 & $168 \cdot 6$ & 273 & $202 \cdot 7$ & 317 & $237 \cdot 6$ & 361 & $272 \cdot 9$ \\
\hline 142 & $10 \cdot 2 \cdot 8$ & 186 & $136 \cdot 2$ & 230 & 169.4 & 274 & $203 \cdot 5$ & 318 & $228 \cdot 4$ & 362 & $273 \cdot 8$ \\
\hline 143 & $103 \cdot 6$ & 187 & 136.9 & 231 & $170 \cdot 1$ & 275 & $204 \cdot 3$ & 319 & $239 \cdot 1$ & 363 & $274^{\circ} 6$ \\
\hline 144 & $104 \cdot 3$ & 188 & $137 \cdot 7$ & 232 & 170.9 & 276 & $205 \cdot 1$ & 320 & 239.9 & 364 & 275.5 \\
\hline 14.5 & $105 \cdot 1$ & 189 & $138 \cdot 5$ & 233 & $171 \cdot 6$ & 277 & $205 \cdot 9$ & 321 & $240^{\circ} 7$ & 365 & $276 \cdot 3$ \\
\hline 146 & $105 \cdot 8$ & 190 & $139 \cdot 2$ & 234 & $172 \cdot 4$ & 278 & $206 \cdot 7$ & 322 & 241.5 & 366 & $277^{\circ} 2$ \\
\hline 147 & $106 \cdot 6$ & 191 & $140^{\circ} 0$ & 235 & $173 \cdot 1$ & 279 & $207 \cdot 5$ & 323 & $242 \cdot 3$ & 367 & $278^{\circ} 0$ \\
\hline 148 & $107 \cdot 3$ & 192 & $140 \cdot 8$ & 236 & 173.9 & 280 & $208 \cdot 3$ & 324 & 243.0 & 368 & $278 \cdot 9$ \\
\hline 149 & $108 \cdot 1$ & 193 & $141 \cdot 5$ & 237 & $174 \%$ & 281 & $209 \cdot 1$ & 325 & $243 \cdot 8$ & 369 & $279 \cdot 7$ \\
\hline 150 & $108 \cdot 8$ & 194 & $142 \cdot 3$ & 238 & $175 \cdot 4$ & 282 & $209 \cdot 9$ & 326 & $244^{\circ} 6$ & 370 & $280 \cdot 5$ \\
\hline 151 & $109 \cdot 6$ & 195 & $143 \cdot 1$ & 239 & $176 \cdot 2$ & 283 & $210 \cdot 7$ & 327 & 245.4 & 371 & $281 \cdot 4$ \\
\hline 152 & $110 \cdot 4$ & 196 & $143 \cdot 8$ & 240 & 176.9 & 284 & $211 \cdot 5$ & 328 & $246 \cdot 1$ & 372 & $282 \cdot 3$ \\
\hline 153 & $111 \cdot 1$ & 197 & $144 \cdot 6$ & 241 & $177 \cdot 7$ & 285 & $212 \cdot 3$ & 329 & $246 \cdot 9$ & 373 & $283 \cdot 1$ \\
\hline 154 & $111 \cdot 9$ & 198 & $145 \cdot 4$ & 242 & 178.5 & 286 & $213 \cdot 1$ & 330 & $247 \cdot 7$ & 374 & $284^{\circ} 0$ \\
\hline 155 & $112 \cdot 6$ & 199 & $146 \cdot 2$ & 243 & $179 \cdot 3$ & 287 & 213.9 & 331 & $248 \cdot 5$ & 375 & $284^{\circ} 8$ \\
\hline 156 & 113.4 & 200 & 146.9 & 244 & $180 \cdot 1$ & 288 & $214 \cdot 7$ & 332 & $249 \cdot 3$ & 376 & $285 \cdot 7$ \\
\hline 157 & $114 \cdot 1$ & 201 & $147 \cdot 7$ & 245 & 180.9 & 289 & $215 \cdot 5$ & 333 & $250 \cdot 1$ & 377 & 286.5 \\
\hline 158 & $114 \cdot 9$ & 202 & $148 \cdot 4$ & 246 & $181^{\circ} 6$ & 290 & $216 \cdot 3$ & 334 & $250 \cdot 9$ & 378 & $287 \cdot 4$ \\
\hline 159 & 115.7 & 203 & $149 \cdot 2$ & 247 & 182.4 & 291 & $217 \cdot 1$ & 335 & $251 \cdot 7$ & 379 & $288 \cdot 2$ \\
\hline 160 & $116 \cdot 4$ & 204 & 149.9 & 248 & $183 \cdot 2$ & 292 & $217 \cdot 9$ & 336 & $252 \cdot 5$ & 380 & $289 \cdot 1$ \\
\hline 161 & $117 \cdot 2$ & 205 & $150 \cdot 7$ & 249 & $184^{\circ} 0$ & 293 & $218 \cdot 7$ & 337 & $253 \cdot 3$ & 381 & $289 \cdot 9$ \\
\hline 162 & $117 \cdot 9$ & 206 & $151 \cdot 4$ & 250 & $184 \cdot 8$ & 294 & $219 \cdot 5$ & 338 & $254: 2$ & 382 & $290 \cdot 8$ \\
\hline 163 & $118 \cdot 7$ & 207 & $152 \cdot 2$ & 251 & $185^{\circ} 6$ & 295 & $220 \cdot 3$ & 339 & $255^{\circ} 0$ & 383 & $291 \cdot 6$ \\
\hline 164 & $119 \cdot 4$ & 208 & 152.9 & 252 & $186 \cdot 3$ & 296 & $221 \cdot 2$ & 340 & $255 \cdot 8$ & 384 & $292 \cdot 5$ \\
\hline 165 & $120 \cdot 2$ & 209 & $153 \cdot 7$ & 253 & $187 \cdot 1$ & 297 & $222 \cdot 0$ & 341 & $256 \cdot 6$ & 385 & $293 \cdot 3$ \\
\hline 166 & $120 \cdot 9$ & 210 & $154^{\circ} 4$ & 254 & $187 \cdot 9$ & 298 & $222 \cdot 8$ & 342 & $257 \cdot 4$ & 386 & $294: 2$ \\
\hline 167 & $121 \cdot 7$ & 211 & $155 \cdot 2$ & 255 & $188^{\circ} 7$ & 299 & $223 \cdot 6$ & 343 & $258 \cdot 2$ & 387 & $295 \cdot 1$ \\
\hline 168 & $122 \cdot 4$ & 212 & 155.9 & 256 & $189 \cdot 4$ & 300 & $224 \cdot 4$ & 344 & $259 \cdot 0$ & 388 & $295 \cdot 9$ \\
\hline 169 & $123 \cdot 2$ & 213 & $156^{\circ} 7$ & 257 & $190 \cdot 2$ & 301 & $225 \cdot 2$ & 345 & $259 \cdot 8$ & 389 & 296.8 \\
\hline 170 & 123.9 & 214 & $157 \cdot 4$ & 258 & $191^{\circ} 0$ & 302 & $225 \cdot 9$ & 346 & $260 \cdot 7$ & 390 & $297 \cdot 7$ \\
\hline 171 & $124 \cdot 7$ & 215 & $158 \%$ & 259 & $191 \cdot 8$ & 303 & $226 \cdot 7$ & 347 & 261.5 & 391 & $298 \cdot 6$ \\
\hline 172 & $125 \cdot 5$ & 216 & $158 \cdot 9$ & 260 & $192 \cdot 6$ & 304 & $227 \cdot 5$ & 348 & $262 \cdot 3$ & 392 & $299 \cdot 4$ \\
\hline 173 & $1 \cdot 26 \cdot 2$ & 217 & $159 \cdot 7$ & 261 & $193 \cdot 3$ & 305 & $228 \cdot 3$ & 349 & $263 \cdot 1$ & 393 & $300 \cdot 3$ \\
\hline 174 & $127 \cdot 0$ & 218 & $160^{\circ} 4$ & 262 & $194 \cdot 1$ & 306 & $229 \cdot 0$ & 350 & 263.9 & 394 & $301 \cdot 1$ \\
\hline 175 & $127 \cdot 8$ & 219 & $161 \cdot 2$ & 263 & $194^{\circ} 9$ & 307 & $229 \cdot 8$ & 351 & $264 \cdot 7$ & 395 & $302 \cdot 0$ \\
\hline 176 & $128 \cdot 6$ & 220 & $161 \cdot 9$ & 264 & $195 \cdot 7$ & 308 & $230 \cdot 6$ & 352 & 2656 & 396 & $302 \cdot 9$ \\
\hline 177 & $129 \cdot 3$ & 221 & $162 \cdot 7$ & 265 & $196^{\circ} 4$ & 309 & $231 \cdot 4$ & 353 & $266^{\circ} 4$ & 397 & $303 \cdot 7$ \\
\hline 178 & $130 \cdot 1$ & 222 & 163.4 & 266 & $197 \cdot 2$ & 310 & $232 \cdot 1$ & 354 & $267 \cdot 2$ & 398 & $304 \cdot 6$ \\
\hline 179 & $130 \cdot 9$ & 223 & $164 \cdot 2$ & 267 & $198^{\circ} 0$ & 311 & $232 \cdot 9$ & 355 & $268^{\circ} 0$ & 399 & $305 \cdot 4$ \\
\hline 180 & $131 \cdot 6$ & 224 & 164.9 & 268 & $198 \cdot 8$ & 312 & $233 \cdot 7$ & 356 & $268 \cdot 8$ & & \\
\hline 181 & $132 \cdot 4$ & 225 & $165 \cdot 6$ & 269 & 199.5 & 313 & $234 \cdot 5$ & 357 & $269 \cdot 6$ & & \\
\hline 14 & $\cdot 1$ & 226 & 166.4 & 270 & $200 \cdot 3$ & 314 & $235 \cdot 3$ & 358 & 270.4 & & \\
\hline 183 & $133 \cdot 9$ & 227 & $167 \cdot 1$ & 271 & $201 \cdot 1$ & 315 & $236^{\circ} 0$ & 359 & $271 \cdot 3$ & & \\
\hline
\end{tabular}




\section{MILK AND DAIRY PRODUCTS}

Table VI. -For Determining the Amount of Milk-Sugar in Milk from the Refractometer Divisions, according to Dr. R. Braun.

\begin{tabular}{|c|c|c|c|c|c|c|c|}
\hline $\begin{array}{c}\text { Scale } \\
\text { Divisions }\end{array}$ & $\begin{array}{l}\text { Milk- } \\
\text { Sugar. } \\
\%\end{array}$ & $\begin{array}{c}\text { Scale } \\
\text { Divisions }\end{array}$ & $\begin{array}{l}\text { Milk- } \\
\text { Sugar. } \\
\%\end{array}$ & $\begin{array}{c}\text { Scale } \\
\text { Divisions }\end{array}$ & $\begin{array}{c}\text { Milk- } \\
\text { Sugar. } \\
\%\end{array}$ & $\begin{array}{c}\text { Scale } \\
\text { Divisions }\end{array}$ & $\begin{array}{l}\text { Milk- } \\
\text { Sugar. } \\
\%\end{array}$ \\
\hline $3 \cdot 1$ & $1 \cdot 75$ & $6 \cdot 1$ & $3 \cdot 31$ & $9 \cdot 1$ & $4 \cdot 84$ & $12 \cdot 1$ & $6 \cdot 35$ \\
\hline$\cdot 2$ & $1 \cdot 80$ & 2 & $3 \cdot 36$ & 2 & $4 \cdot 89$ & 2 & $6 \cdot 40$ \\
\hline$\cdot 3$ & 1.85 & 3 & $3 \cdot 42$ & •3 & $4 \cdot 95$ & $\cdot 3$ & $6 \cdot 46$ \\
\hline$\cdot 4$ & 1.90 & $\cdot 4$ & $3 \cdot 47$ & $\cdot 4$ & $5 \cdot 00$ & $\cdot 4$ & $6 \cdot 51$ \\
\hline 5 & 1.96 & $\cdot 5$ & $3 \cdot 52$ & $\cdot 5$ & $5 \cdot 05$ & 5 & $6 \cdot 56$ \\
\hline$\cdot 6$ & $2 \cdot 01$ & $\cdot 6$ & $3 \cdot 57$ & $\cdot 6$ & $5 \cdot 10$ & $\cdot 6$ & $6 \cdot 61$ \\
\hline 7 & 2.07 & 7 & $3 \cdot 62$ & 7 & $5 \cdot 15$ & $\cdot 7$ & $6 \cdot 66$ \\
\hline 8 & $2 \cdot 12$ & 8 & 3.67 & $\cdot 8$ & $5 \cdot 20$ & $\cdot 8$ & $6 \cdot 71$ \\
\hline$\cdot 9$ & $2 \cdot 18$ & 9 & $3 \cdot 72$ & 9 & $5 \cdot 25$ & 9 & $6 \cdot 76$ \\
\hline $4 \cdot 0$ & $2 \cdot 23$ & $7 \cdot 0$ & $3 \cdot 77$ & $10 \cdot 0$ & $5 \cdot 30$ & $13 \cdot 0$ & $6 \cdot 81$ \\
\hline$\cdot 1$ & $2 \cdot 29$ & $\cdot 1$ & $3 \cdot 82$ & $\cdot 1$ & $5 \cdot 35$ & $\cdot 1$ & $6 \cdot 86$ \\
\hline$\cdot 2$ & $2 \cdot 35$ & 2 & $3 \cdot 87$ & 2 & $5 \cdot 40$ & 2 & 6.91 \\
\hline$\cdot 3$ & $2 \cdot 40$ & $\cdot 3$ & $3 \cdot 93$ & •3 & $5 \cdot 45$ & 3 & 6.97 \\
\hline$\cdot 4$ & $2 \cdot 45$ & $\cdot 4$ & $3 \cdot 98$ & $\cdot 4$ & $5 \cdot 50$ & $\cdot 4$ & $7 \cdot 02$ \\
\hline$\cdot 5$ & $2 \cdot 50$ & $\cdot 5$ & $4 \cdot 03$ & $\cdot 5$ & $5 \cdot 55$ & 5 & $7 \cdot 07$ \\
\hline$\cdot 6$ & 2.55 & $\cdot 6$ & $4 \cdot 08$ & 6 & $5 \cdot 60$ & 6 & $7 \cdot 12$ \\
\hline 7 & $2 \cdot 60$ & $\cdot 7$ & $4 \cdot 13$ & $\cdot 7$ & $5 \cdot 65$ & 7 & $7 \cdot 17$ \\
\hline 8 & $2 \cdot 65$ & 8 & $4 \cdot 18$ & 8 & $5 \cdot 70$ & 8 & $7 \cdot 22$ \\
\hline .9 & $2 \cdot 70$ & $\cdot 9$ & $4 \cdot 23$ & 9 & $5 \cdot 75$ & $\cdot 9$ & $7 \cdot 27$ \\
\hline $5 \cdot 0$ & $2 \cdot 75$ & $8 \cdot 0$ & $4 \cdot 28$ & $11 \cdot 0$ & $5 \cdot 80$ & $14 \cdot 0$ & $7 \cdot 33$ \\
\hline$\cdot 1$ & $2 \cdot 80$ & $\cdot 1$ & $4 \cdot 33$ & •l & $5 \cdot 85$ & $\cdot 1$ & $7 \cdot 38$ \\
\hline$\cdot 2$ & $2 \cdot 85$ & $\cdot 2$ & $4 \cdot 38$ & $\cdot 2$ & $5 \cdot 90$ & 2 & $7 \cdot 43$ \\
\hline$\cdot 3$ & $2 \cdot 91$ & 3 & $4 \cdot 44$ & 3 & $5 \cdot 95$ & ·3 & $7 \cdot 48$ \\
\hline$\cdot 4$ & $2 \cdot 96$ & $\cdot 4$ & $4 \cdot 49$ & 4 & $6 \cdot 00$ & $\cdot 4$ & $7 \cdot 53$ \\
\hline$\cdot 5$ & $3 \cdot 01$ & $\cdot 5$ & $4 \cdot 54$ & $\cdot 5$ & $6 \cdot 05$ & 5 & $7 \cdot 58$ \\
\hline 6 & 3.06 & $\cdot 6$ & 4.59 & $\cdot 6$ & $6 \cdot 10$ & $\cdot 6$ & $7 \cdot 63$ \\
\hline$\cdot 7$ & $3 \cdot 11$ & .7 & $4 \cdot 64$ & $\cdot 7$ & $6 \cdot 15$ & $\cdot 7$ & $7 \cdot 68$ \\
\hline 8 & $3 \cdot 16$ & 8 & $4 \cdot 639$ & $\cdot 8$ & $6 \cdot 20$ & $\cdot 8$ & $7 \cdot 73$ \\
\hline$\cdot 9$ & $3 \cdot 21$ & $\cdot 9$ & $4 \cdot 74$ & $\cdot 9$ & $6 \cdot 25$ & $\cdot 9$ & $7 \cdot 78$ \\
\hline $6 \cdot 0$ & $3 \cdot 26$ & $9 \cdot 0$ & $4 \cdot 79$ & $12 \cdot 0$ & $6 \cdot 30$ & $15 \cdot 0$ & $7 \cdot 84$ \\
\hline
\end{tabular}


Table Vila.-For Calculating the Amount of Total Solids - in Milk fron the Specific Gravity a and tile Percentage of Fat $f$, according to Fleischimann.

\begin{tabular}{|c|c|c|c|c|c|}
\hline $\begin{array}{c}s \\
\text { Thousandths }\end{array}$ & $\begin{array}{c}2 \cdot 665 \\
100 s-100 \\
s\end{array}$ & $\stackrel{8}{\text { Thousandths }}$ & $\begin{array}{c}2 \cdot 665 \\
100 s-100 \\
s\end{array}$ & $\stackrel{8}{\text { Thousandths }}$ & $\begin{array}{c}2 \cdot 665 . \\
1008-100 \\
8\end{array}$ \\
\hline $\begin{array}{r}28 \cdot 0 \\
\cdot 1 \\
\cdot 2\end{array}$ & $\begin{array}{l}7 \cdot 259 \\
7 \cdot 284 \\
7 \cdot 309\end{array}$ & $\begin{array}{r}31 \cdot 0 \\
\cdot 1 \\
\cdot 2\end{array}$ & $\begin{array}{l}8 \cdot 013 \\
8 \cdot 038 \\
8 \cdot 063\end{array}$ & $\begin{array}{r}34 \cdot 0 \\
\cdot 1 \\
\cdot 2\end{array}$ & $\begin{array}{l}8 \cdot 763 \\
8 \cdot 788 \\
8 \cdot 813\end{array}$ \\
\hline $\begin{array}{l}3 \\
\cdot 4 \\
\cdot 5\end{array}$ & $\begin{array}{l}7 \cdot 334 \\
7 \cdot 360 \\
7 \cdot 385\end{array}$ & $\begin{array}{l}\because 3 \\
\cdot 4 \\
\cdot 5\end{array}$ & $\begin{array}{l}8 \cdot 088 \\
8 \cdot 113 \\
8 \cdot 138\end{array}$ & $\begin{array}{r}\cdot 3 \\
\cdot 4 \\
\cdot 5\end{array}$ & $\begin{array}{l}8 \cdot 838 \\
8 \cdot 863 \\
8 \cdot 888\end{array}$ \\
\hline $\begin{array}{l}6 \\
.7 \\
.8\end{array}$ & $\begin{array}{l}7 \cdot 410 \\
7 \cdot 435 \\
7 \cdot 460\end{array}$ & $\begin{array}{l}.6 \\
.7 \\
.8\end{array}$ & $\begin{array}{l}8 \cdot 163 \\
8188 \\
8 \cdot 213\end{array}$ & $\begin{array}{l}\cdot 6 \\
\cdot 7 \\
\cdot 8\end{array}$ & $\begin{array}{l}8 \cdot 912 \\
8 \cdot 937 \\
8 \cdot 962\end{array}$ \\
\hline $\begin{array}{r}\cdot 9 \\
29 \cdot 0 \\
\cdot 1\end{array}$ & $\begin{array}{l}7 \cdot 485 \\
7 \cdot 511 \\
7 \cdot 536\end{array}$ & $\begin{array}{r}\cdot 9 \\
32 \cdot 0 \\
\cdot 1\end{array}$ & $\begin{array}{l}8 \cdot 239 \\
8 \cdot 264 \\
8 \cdot 289\end{array}$ & $\begin{array}{r}\cdot 9 \\
35 \cdot 0 \\
\cdot 1\end{array}$ & $\begin{array}{l}8 \cdot 987 \\
9 \cdot 012 \\
9 \cdot 037\end{array}$ \\
\hline $\begin{array}{l}\cdot 2 \\
\cdot 3 \\
\cdot 4\end{array}$ & $\begin{array}{l}7 \cdot 561 \\
7 \cdot 586 \\
7 \cdot 611\end{array}$ & $\begin{array}{l}\cdot 2 \\
\cdot 3 \\
\cdot 4\end{array}$ & $\begin{array}{l}8 \cdot 314 \\
8 \cdot 339 \\
8 \cdot 364\end{array}$ & $\begin{array}{l}\cdot 2 \\
\cdot 3 \\
\cdot 4\end{array}$ & $\begin{array}{l}9 \cdot 062 \\
9 \cdot 087 \\
9 \cdot 111\end{array}$ \\
\hline $\begin{array}{l}5 \\
.6 \\
.7\end{array}$ & $\begin{array}{l}7 \cdot 636 \\
7 \cdot 662 \\
7 \cdot 687\end{array}$ & $\begin{array}{l}\cdot 5 \\
6 \\
.7\end{array}$ & $\begin{array}{l}8 \cdot 389 \\
8 \cdot 414 \\
8 \cdot 439\end{array}$ & $\begin{array}{l}.5 \\
.6 \\
.7\end{array}$ & $\begin{array}{l}9 \cdot 136 \\
9 \cdot 161 \\
9 \cdot 186\end{array}$ \\
\hline $\begin{array}{r}8 \\
\cdot 9 \\
30.0\end{array}$ & $\begin{array}{l}7 \cdot 712 \\
7 \cdot 737 \\
7 \cdot 762\end{array}$ & $\begin{array}{r}\cdot 8 \\
\cdot 9 \\
33 \cdot 0\end{array}$ & $\begin{array}{l}8 \cdot 464 \\
8 \cdot 489 \\
8 \cdot 514\end{array}$ & $\begin{array}{r}\cdot 8 \\
\cdot 9 \\
36 \cdot 0\end{array}$ & $\begin{array}{l}9 \cdot 211 \\
9 \cdot 236 \\
9 \cdot 261\end{array}$ \\
\hline $\begin{array}{l}\cdot 1 \\
\cdot 2 \\
\cdot 3\end{array}$ & $\begin{array}{l}7 \cdot 787 \\
7 \cdot 812 \\
7 \cdot 837\end{array}$ & $\begin{array}{l}\cdot 1 \\
\cdot 2 \\
\cdot 3\end{array}$ & $\begin{array}{l}8 \cdot 539 \\
8 \cdot 563 \\
8 \cdot 588\end{array}$ & $\begin{array}{l}\cdot 1 \\
\cdot 2 \\
\cdot 3\end{array}$ & $\begin{array}{l}9 \cdot 285 \\
9 \cdot 310 \\
9 \cdot 335\end{array}$ \\
\hline $\begin{array}{l}\cdot 4 \\
\cdot 5 \\
\cdot 6\end{array}$ & $\begin{array}{l}7 \cdot 863 \\
7 \cdot 888 \\
7 \cdot 913\end{array}$ & $\begin{array}{l}\cdot 4 \\
\cdot 5 \\
.6\end{array}$ & $\begin{array}{l}8 \cdot 613 \\
8638 \\
8 \cdot 663\end{array}$ & $\begin{array}{l}\cdot 4 \\
\cdot 5 \\
.6\end{array}$ & $\begin{array}{l}9 \cdot 360 \\
9 \cdot 385 \\
9 \cdot 409\end{array}$ \\
\hline $\begin{array}{l}\cdot 7 \\
.8 \\
.9\end{array}$ & $\begin{array}{l}7 \cdot 938 \\
7 \cdot 963 \\
7 \cdot 988\end{array}$ & $\begin{array}{l}\cdot 7 \\
\cdot 8 \\
\cdot 9\end{array}$ & $\begin{array}{l}8 \cdot 688 \\
8 \cdot 713 \\
8 \cdot 738\end{array}$ & $\begin{array}{l}\cdot 7 \\
\cdot 8 \\
\cdot 9\end{array}$ & $\begin{array}{l}9 \cdot 434 \\
9 \cdot 459 \\
9 \cdot 484\end{array}$ \\
\hline
\end{tabular}




\section{MILK AND DAIRY PRODUCTS}

Table VIIb.-For Calculating the Amount of Total Solids in Milk from the Specific Gravity \& and the Percentage of

Fat $f$, according to Fleischmann.

\begin{tabular}{|c|c|c|c|c|c|c|c|c|c|c|c|}
\hline$f$ & $1 \cdot 2 . f$ & $f$ & $1 \cdot 2 . f$ & $f$ & $1.2 . f$ & $f$ & $1 \cdot 2 . f$ & $f$ & $1 \cdot 2 . f$ & $f$ & $1 \cdot 2 . f$ \\
\hline 50 & 3.000 & $3 \cdot 00$ & $3 \cdot 600$ & 3.50 & $4 \cdot 200$ & $4 \cdot 00$ & $4 \cdot 800$ & $4 \cdot 50$ & $5 \cdot 400$ & $5 \cdot 00$ & 6.000 \\
\hline .51 & $3 \cdot 012$ & $\cdot 01$ & 612 & .51 & $4 \cdot 212$ & .01 & $4 \cdot 812$ & 51 & $5 \cdot 412$ & $\cdot 01$ & 6.012 \\
\hline$\cdot 52$ & $3 \cdot 024$ & $\cdot 02$ & $3 \cdot 624$ & $\cdot 52$ & $4 \cdot 224$ & $\cdot 02$ & $4 \cdot 824$ & 52 & $5 \cdot 424$ & $\cdot 02$ & 6.024 \\
\hline .53 & $3 \cdot 036$ & $\bullet 03$ & $3 \cdot 636$ & .53 & & .03 & $4 \cdot 836$ & 53 & 5.436 & $\cdot 03$ & $6 \cdot 036$ \\
\hline .54 & $3 \cdot 048$ & .04 & $3 \cdot 648$ & .54 & $4 \cdot 248$ & .04 & $4 \cdot 848$ & .54 & 5.448 & .04 & $6 \cdot 048$ \\
\hline •5̃ & $3 \cdot 060$ & $\cdot 05$ & $3 \cdot 660$ & $\cdot 55$ & $4 \cdot 260$ & $\cdot 05$ & $4 \cdot 860$ & 55 & $5 \cdot 460$ & $\cdot 05$ & 6.060 \\
\hline$\cdot 56$ & $3 \cdot 072$ & $\cdot 06$ & $3 \cdot 672$ & $\cdot 56$ & $4 \cdot 272$ & $\cdot 06$ & $4 \cdot 872$ & •56 & $5 \cdot 472$ & $\cdot 06$ & 6.072 \\
\hline 57 & $3 \cdot 084$ & $\cdot 07$ & $3 \cdot 684$ & .57 & $4 \cdot 284$ & .07 & $4 \cdot 884$ & 57 & $5 \cdot 484$ & .07 & $6 \cdot 084$ \\
\hline .58 & $3 \cdot 096$ & $\cdot 08$ & $3 \cdot 696$ & 58 & $4 \cdot 296$ & .08 & $4 \cdot 896$ & 58 & & $\cdot 08$ & 6.096 \\
\hline .59 & $3 \cdot 108$ & .09 & $3 \cdot 708$ & .59 & $4 \cdot 308$ & .09 & $4 \cdot 908$ & .59 & $5 \cdot 508$ & .09 & $6 \cdot 108$ \\
\hline $2 \cdot 60$ & $3 \cdot 120$ & $3 \cdot 10$ & 3.720 & $3 \cdot 60$ & $4 \cdot 320$ & $4 \cdot 10$ & $4 \cdot 920$ & $4 \cdot 60$ & $5 \cdot 520$ & $5 \cdot 10$ & $6 \cdot 120$ \\
\hline 61 & $3 \cdot 132$ & $\cdot 11$ & $3 \cdot 732$ & 61 & $4 \cdot 332$ & $\cdot 11$ & 4.932 & $\cdot 61$ & $5 \cdot 532$ & $\cdot 11$ & $6 \cdot 132$ \\
\hline$\cdot 62$ & $3 \cdot 144$ & $\cdot 12$ & $3 \cdot 744$ & $\cdot 62$ & $4 \cdot 344$ & -12 & $4 \cdot 944$ & 62 & $5 \cdot 544$ & $\cdot 12$ & $6 \cdot 144$ \\
\hline$\cdot 63$ & $3 \cdot 156$ & $\cdot 13$ & $3 \cdot 756$ & $\cdot 63$ & $4 \cdot 356$ & 13 & $4 \cdot 956$ & $\cdot 63$ & 5 & $\cdot 13$ & $6 \cdot 156$ \\
\hline$\cdot 64$ & $3 \cdot 168$ & $\cdot 14$ & $3 \cdot 768$ & $\cdot 64$ & $4 \cdot 368$ & $\cdot 14$ & $4 \cdot 968$ & $\cdot 64$ & & $\cdot 14$ & $6 \cdot 168$ \\
\hline 65 & $3 \cdot 180$ & $\cdot 15$ & $3 \cdot 780$ & •65 & $4 \cdot 380$ & $\cdot 15$ & $4 \cdot 980$ & $\cdot 65$ & & $\cdot 15$ & $6 \cdot 180$ \\
\hline$\cdot 66$ & $3 \cdot 192$ & $\cdot 16$ & $3 \cdot 792$ & $\cdot 66$ & $4 \cdot 392$ & $\cdot 16$ & 4.992 & $\cdot 66$ & $5 \cdot 592$ & $\cdot 16$ & $6 \cdot 192$ \\
\hline 67 & $3 \cdot 204$ & $\cdot 17$ & $3 \cdot 804$ & $\cdot 67$ & $4 \cdot 404$ & $\cdot 17$ & $5 \cdot 004$ & 67 & & $\cdot 17$ & $6 \cdot 204$ \\
\hline$\cdot 68$ & $3 \cdot 216$ & $\cdot 18$ & $3 \cdot 816$ & $\cdot 68$ & $4 \cdot 416$ & $\cdot 18$ & $5 \bullet 016$ & $\cdot 68$ & & $\cdot 18$ & 6.216 \\
\hline$\cdot 69$ & $3 \cdot 228$ & $\cdot 19$ & $3 \cdot 828$ & $\cdot 69$ & $4 \cdot 428$ & $\cdot 19$ & $5 \cdot 028$ & $\cdot 69$ & & $\cdot 19$ & $6 \cdot 228$ \\
\hline $2 \cdot 70$ & $3 \cdot 240$ & 3.20 & $3 \cdot 840$ & $3 \cdot 70$ & $4 \cdot+40$ & 4.20 & $5 \cdot 040$ & $4 \cdot 70$ & & $5 \cdot 20$ & 6.240 \\
\hline 71 & $3 \cdot 252$ & $\cdot 21$ & $3 \cdot 852$ & $\cdot 71$ & $4 \cdot 452$ & $\cdot 21$ & $5 \cdot 052$ & 71 & 5. & .21 & $6 \cdot 252$ \\
\hline .72 & $3 \cdot 264$ & $\cdot 22$ & $3 \cdot 864$ & $\cdot 72$ & $4 \cdot 464$ & $\cdot 22$ & $5 \cdot 064$ & 72 & & $\cdot 2 \cdot 2$ & $6 \cdot 264$ \\
\hline$\cdot 73$ & $3 \cdot 276$ & .23 & $3 \cdot 876$ & $\cdot 73$ & $4 \cdot 476$ & "23 & $5 \cdot 076$ & $\cdot 73$ & & 23 & $6 \cdot 276$ \\
\hline 74 & $3 \cdot 288$ & '24 & $3 \cdot 888$ & $\cdot 74$ & $4 \cdot 488$ & $\cdot 24$ & $5 \cdot$ & $\cdot 74$ & & $\cdot 24$ & $6 \cdot 288$ \\
\hline 75 & $3 \cdot 300$ & 25 & 3.900 & .75 & $4 \cdot 500$ & $\cdot 25$ & $5 \cdot 100$ & $\cdot 75$ & & $\cdot 25$ & $6 \cdot 300$ \\
\hline$\cdot 76$ & $3 \cdot 312$ & $\cdot 26$ & 3.912 & 76 & $4 \cdot 512$ & .26 & $5 \cdot 112$ & $\cdot 76$ & & $\cdot 26$ & $6 \cdot 312$ \\
\hline$\cdot 77$ & $3 \cdot 324$ & $\cdot 27$ & 3.924 & 77 & $4 \cdot 524$ & -27 & 24 & 77 & 5 & 27 & $6 \cdot 324$ \\
\hline 78 & $3 \cdot 336$ & $\cdot 28$ & 3.936 & $\cdot 78$ & $4 \cdot 536$ & .28 & $\cdot 136$ & $\cdot 78$ & $5^{\circ}$ & 28 & 6.336 \\
\hline 79 & $3 \cdot 348$ & 29 & 3.948 & .79 & $4 \cdot 548$ & 29 & & 79 & & 29 & $6: 348$ \\
\hline $2 \cdot 80$ & $3 \cdot 360$ & $3 \cdot 30$ & 3.960 & $3 \cdot 80$ & $4 \cdot 560$ & $4 \cdot 30$ & 30 & $4 \cdot 80$ & & $5 \cdot 30$ & $6: 360$ \\
\hline$\cdot 81$ & $3 \cdot 372$ & $\cdot 31$ & 3.972 & 81 & 4.572 & 31 & $5 \cdot 172$ & $\cdot 81$ & $5 \cdot 772$ & 31 & 6.372 \\
\hline 82 & 3:384 & $\cdot 32$ & 3.984 & $\cdot 82$ & $4 \cdot 584$ & 32 & & $\cdot 82$ & & 32 & $6 \cdot 384$ \\
\hline$\cdot 83$ & 96 & 33 & 3 & 83 & $4 \cdot 5$ & $\cdot 33$ & & •83 & & 33 & $6: 396$ \\
\hline 84 & 3 & $\cdot 34$ & 4.0 & $\cdot 84$ & $4 \cdot 6$ & 34 & & .84 & & ·34 & 6. \\
\hline$\cdot 85$ & 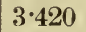 & $\cdot 35$ & $4 \cdot 0$ & 85 & & 35 & & •85 & & 35 & 6. \\
\hline 86 & & $\cdot 36$ & 4 & $\cdot 86$ & $4 \cdot 6$ & $\cdot 36$ & $5 \%$ & $\cdot 86$ & 5 & $\cdot 36$ & 6.432 \\
\hline 87 & & 37 & 4 & 87 & $4 \cdot 6$ & 37 & & 87 & & 37 & \\
\hline 88 & & 38 & & $\cdot 88$ & & ·38 & & ·88 & & 38 & 6 \\
\hline$\cdot 89$ & & $\cdot 39$ & & $\cdot 89$ & & ·39 & & '89 & & ·39 & 6.468 \\
\hline $2 \cdot 90$ & 180 & $3 \cdot 40$ & 30 & 3.90 & $4^{\cdot}$ & $4 \cdot 40$ & & $4 \cdot 90$ & & $5 \cdot 40$ & \\
\hline & & -41 & & .91 & & $\cdot 41$ & & .91 & & $\cdot 41$ & 6 \\
\hline .92 & & $\cdot 42$ & & $\cdot 92$ & $4 \cdot 704$ & $\cdot 42$ & $5:$ & .92 & & $\cdot 42$ & 6.504 \\
\hline 93 & & $\cdot 43$ & & .93 & $4 \cdot 716$ & $\cdot 43$ & & .98 & & $\cdot 43$ & \\
\hline$\because$ & & $\cdot 44$ & & $\cdot 94$ & & $\cdot 44$ & & .94 & & $\cdot 44$ & 6. \\
\hline 95 & 40 & $\cdot 45$ & $4 \cdot 140$ & 95 & $4 \cdot 740$ & $\cdot 45$ & & 95 & & $\cdot 45$ & 6.540 \\
\hline & & $\cdot 46$ & & $\cdot 96$ & & $\cdot 46$ & & $\cdot 96$ & & •46 & 6.552 \\
\hline 9 & & $\cdot 47$ & & $\cdot 97$ & & $\cdot 47$ & & .97 & & $\cdot 47$ & 6.564 \\
\hline$\cdot 98$ & 3. & $\cdot 48$ & $4 \cdot 176$ & .98 & $4 \cdot 776$ & $\cdot 48$ & & .98 & & .48 & 6.576 \\
\hline$\cdot 99$ & $3 \cdot 588$ & $\cdot 49$ & $4 \cdot 188$ & $\cdot 99$ & $4 \cdot 788$ & $\cdot 49$ & $5 \cdot 388$ & $\cdot 99$ & $5 \cdot 988$ & $\bullet 49$ & 6.588 \\
\hline & & & 1 & 02 & 3 & $\begin{array}{l}4 \\
005\end{array}$ & $\begin{array}{c}5 \\
0.006\end{array}$ & $\begin{array}{c}6 \\
.007\end{array}$ & $\begin{array}{l}7 \\
.008\end{array}$ & \begin{tabular}{|c|}
8 \\
0.010
\end{tabular} & $\begin{array}{c}9 \\
01\end{array}$ \\
\hline
\end{tabular}




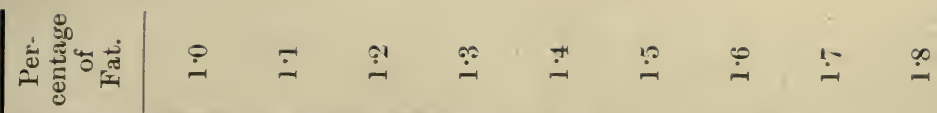

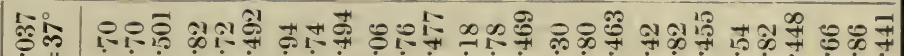

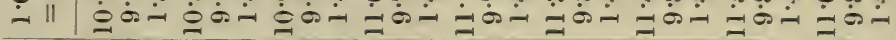

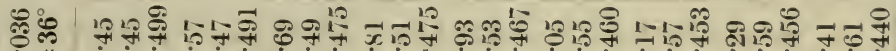

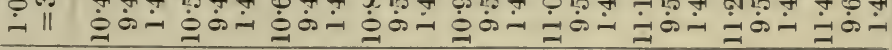

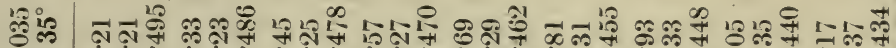
- II

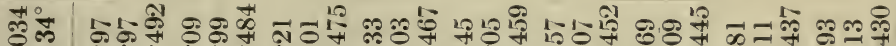
-

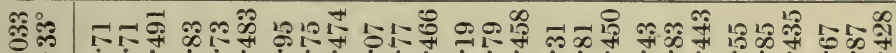

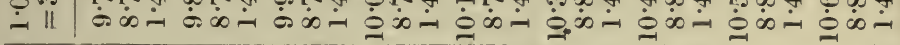

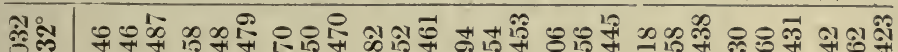

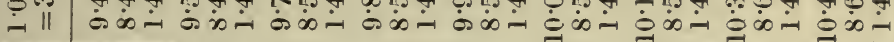

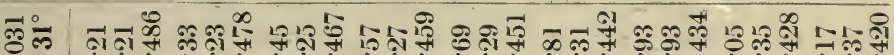

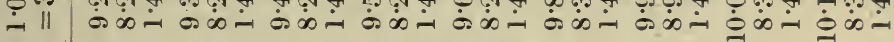

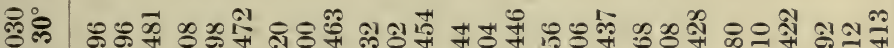

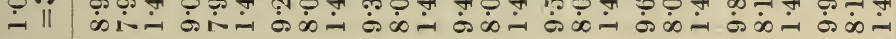

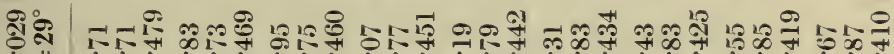

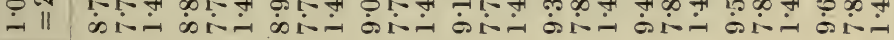

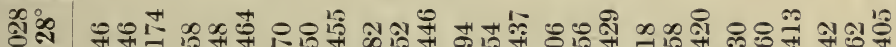

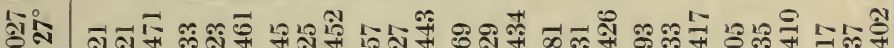

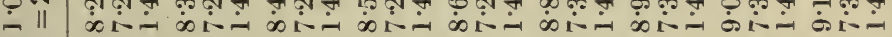

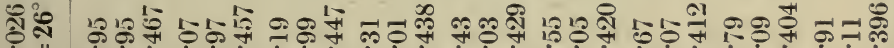

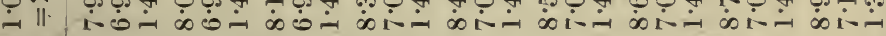
成啳

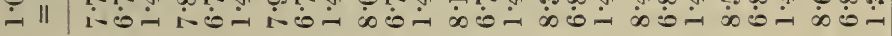

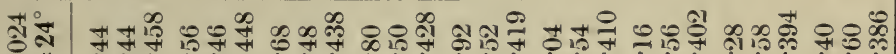

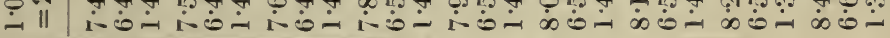

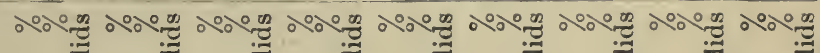
: : : : : : : : : : : : : : : : : : : : : :

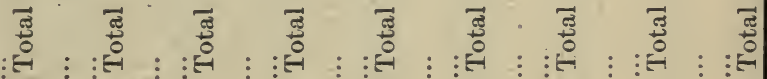
等

펴

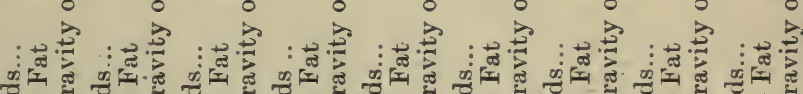

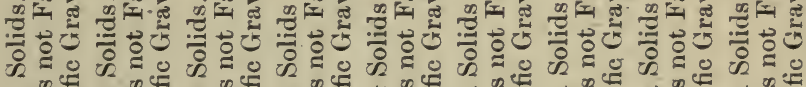
So 0

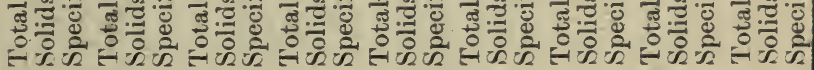

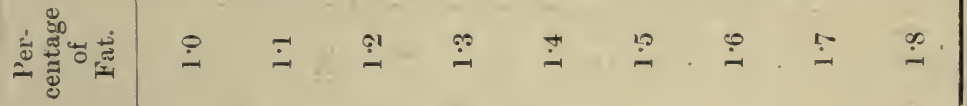




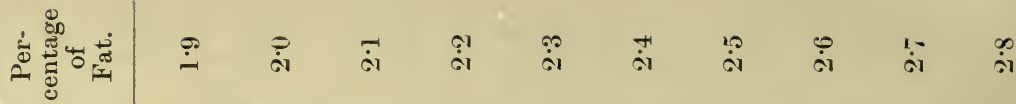

๙ூ๊

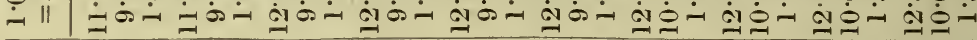

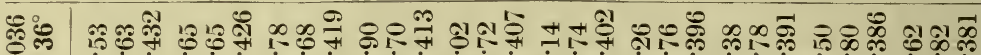

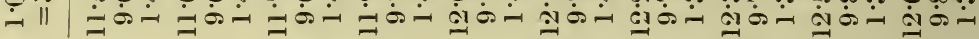

ผิง

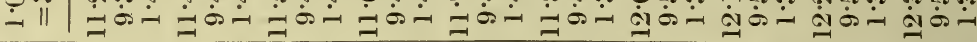

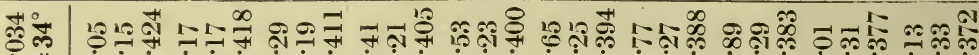

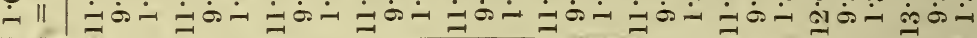

\% \%

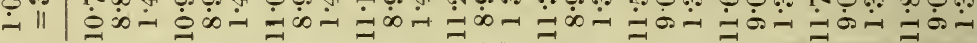
ํํㅇำ

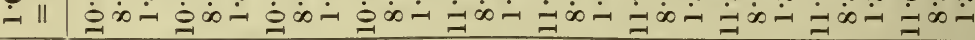

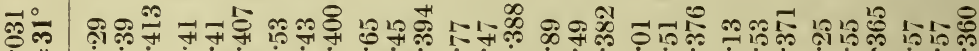

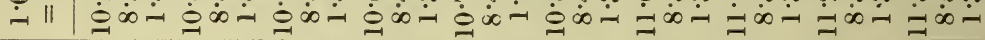

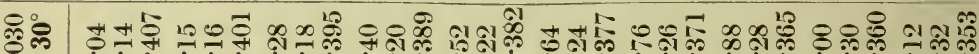

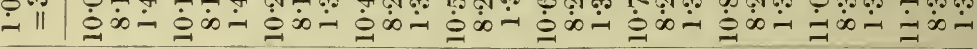

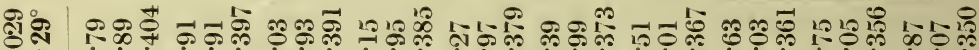

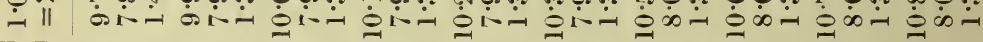

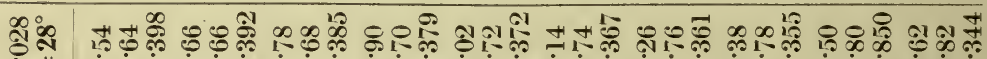

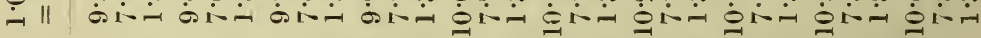

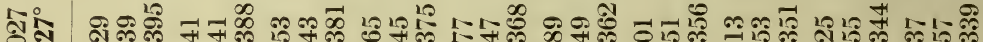

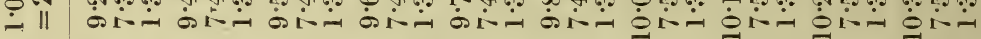
จิ์

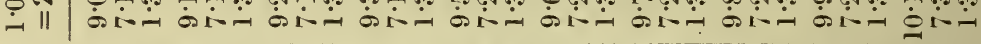

ริำ

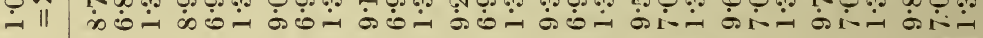

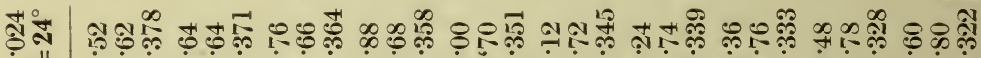

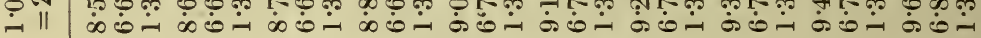

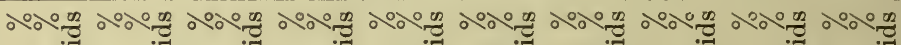

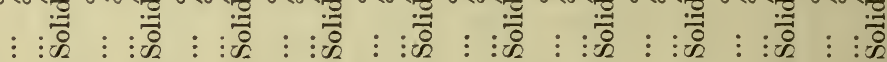

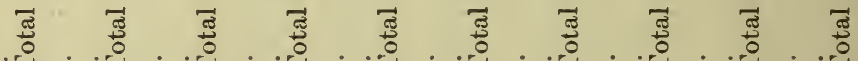
$\vdots:$ : $:$ : $:$ : $:$ : $:$ : .

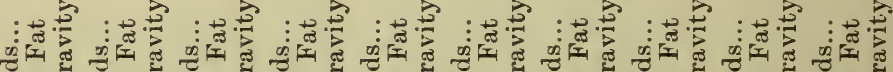

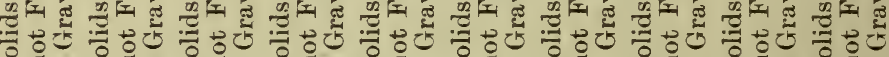
次 వై

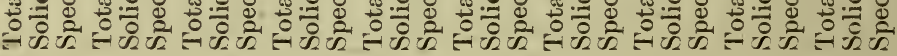

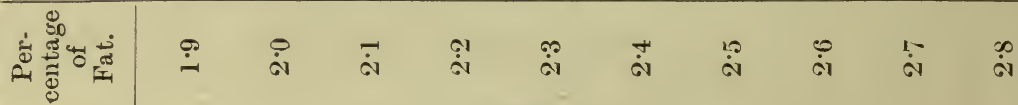




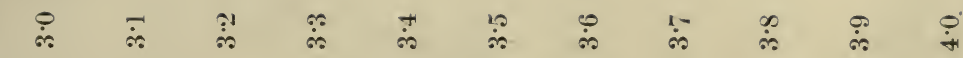

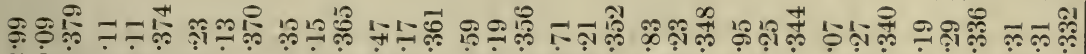

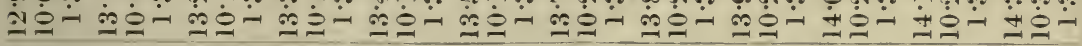
나

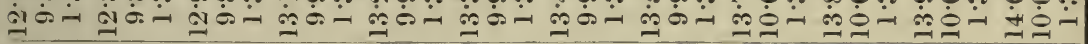
ๆ

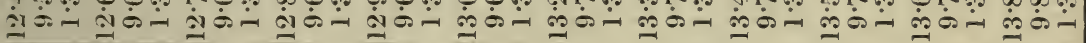
หิด

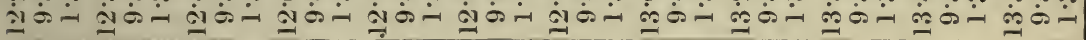

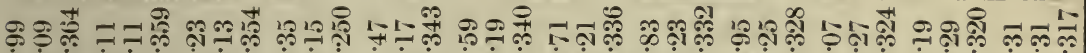

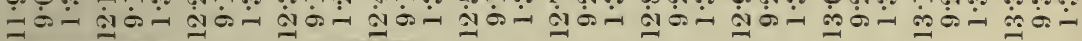
स+⿴囗十

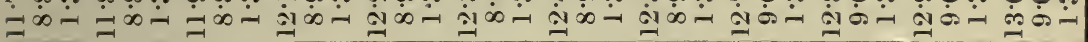

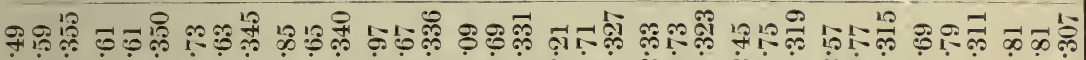

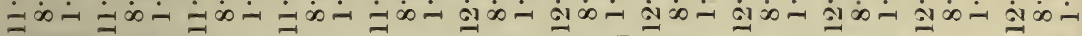
สี่

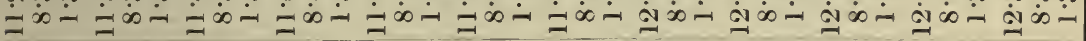

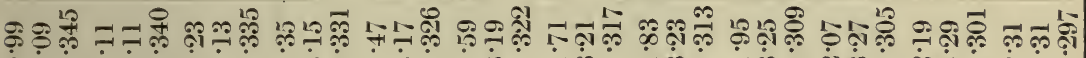

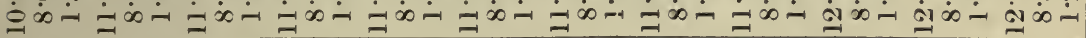

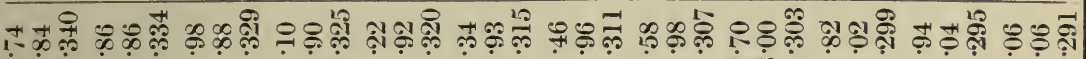

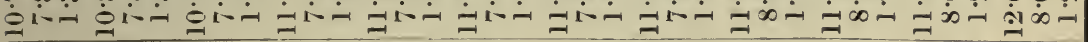
ㅇํำ

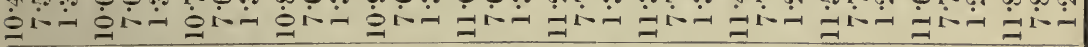
สิๆ ศิ

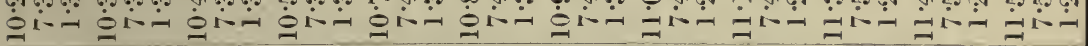
๙

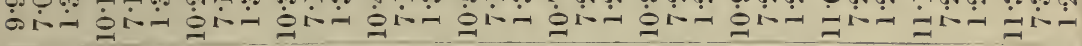

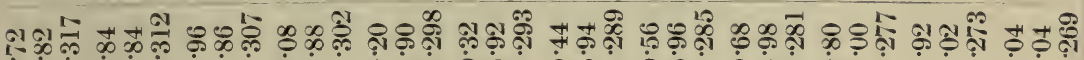

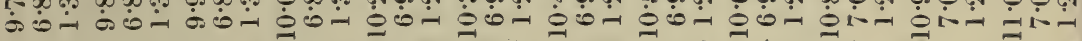

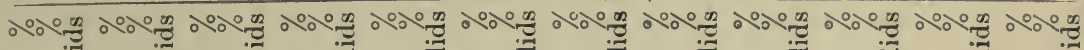

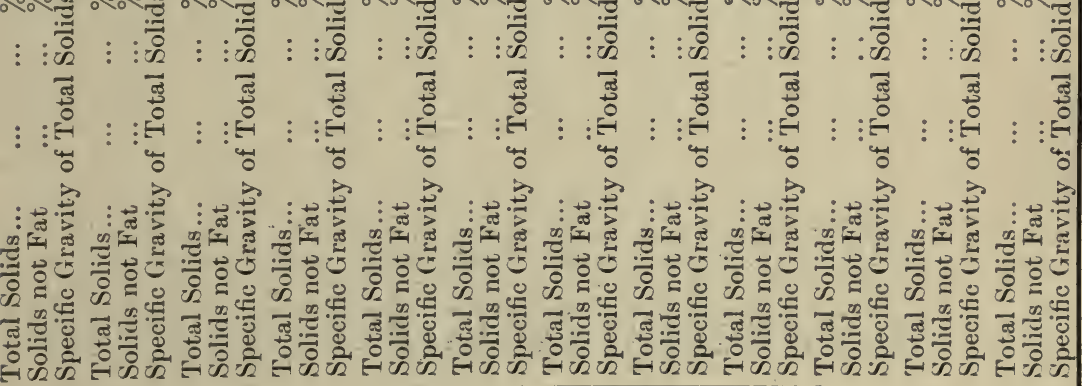
$\stackrel{\leftrightarrow}{\leftrightarrow}$ $\ddot{\therefore} \quad \dot{\leftrightarrow}$ $\stackrel{\leftrightarrow}{\leftrightarrow}$ $\dot{1}$ is $\dot{n} \quad \dot{0}$ $\infty$ ஸे. 


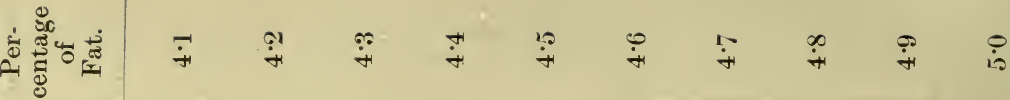

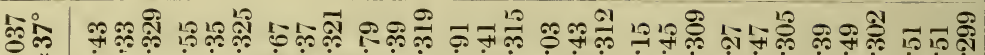

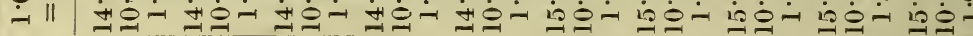

๓

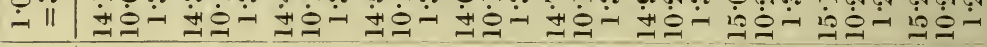

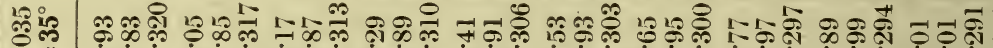

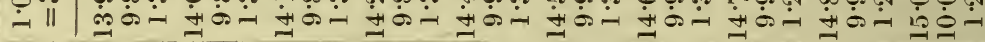
范苛

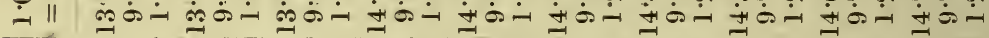

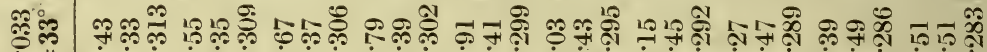

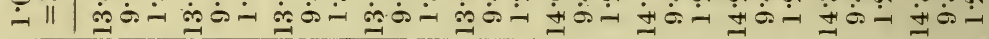

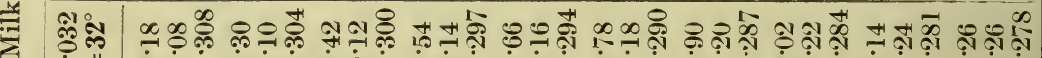

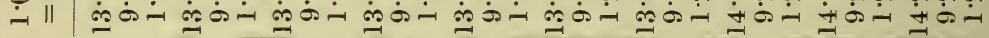

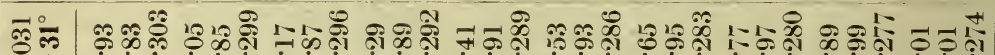
- II

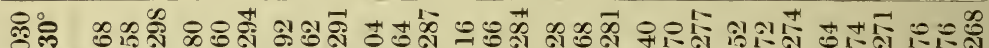

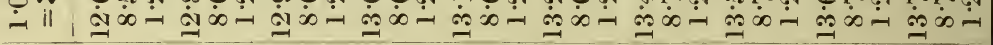

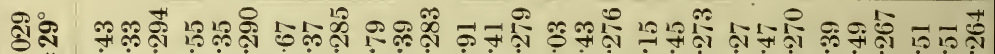

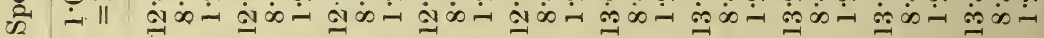

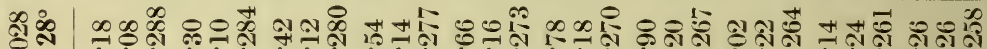

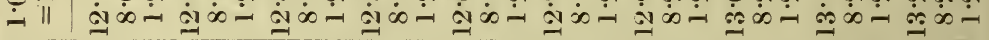
송ํㅕㄴ

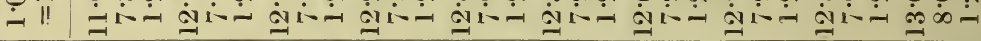

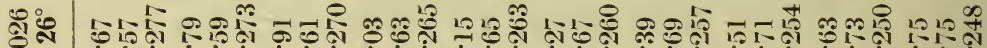

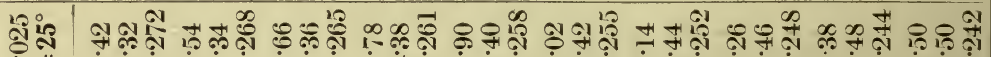

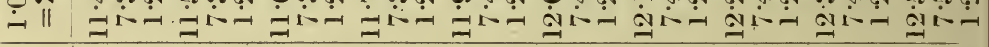

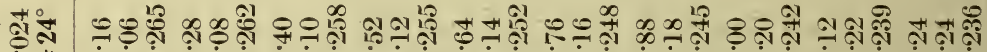

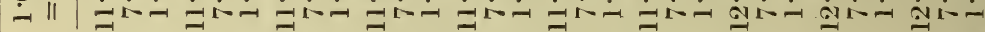

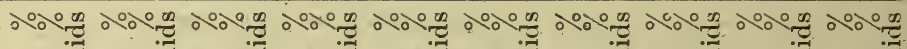
: : : : : : : :

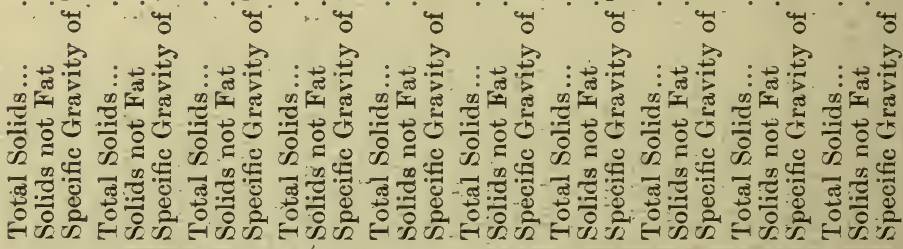

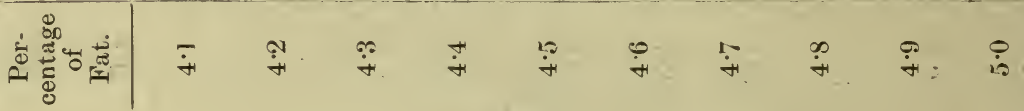


Table IX.-For Calculativg the Variations of the Critical Temperature for Alcohol of Different Specific Gravities, Acconding to Crismer.

\begin{tabular}{|c|c|c|c|c|c|}
\hline $\begin{array}{l}\text { Sp. gr. of } \\
\text { alcohol at } \\
15 \cdot 5^{\circ} \mathrm{C} \text {. }\end{array}$ & Water \% & $\begin{array}{c}\text { Critical } \\
\text { temperature } \\
\text { degrees. }\end{array}$ & $\begin{array}{l}\text { Sp. gr. of } \\
\text { alcohol at } \\
15.5^{\circ} \mathrm{C} \text {. }\end{array}$ & Water \% & $\begin{array}{c}\text { Critical } \\
\text { temperature } \\
\text { degrees. }\end{array}$ \\
\hline $0 \cdot 7938$ & 0 & $48 \cdot 6$ & $0 \cdot 7962$ & $0 \cdot 768$ & $53 \cdot 06$ \\
\hline 0.7939 & 0.032 & $48 \cdot 78$ & 0.7963 & 0.800 & $53 \cdot 24$ \\
\hline $0 \cdot 7940$ & 0.06 & $48 \cdot 97$ & 0.7964 & $0 \cdot 832$ & $53 \cdot 42$ \\
\hline $0 \cdot 7941$ & 0.096 & $49 \cdot 15$ & $0 \cdot 7965$ & $0 \cdot 864$ & $53 \cdot 61$ \\
\hline 0.7942 & $0 \cdot 128$ & $49 \cdot 34$ & $0 \cdot 7966$ & 0.896 & $53 \cdot 79$ \\
\hline 07943 & $0 \cdot 161$ & $49 \cdot 53$ & $0 \cdot 7967$ & 0.928 & $53 \cdot 98-54$ \\
\hline $0 \cdot 7944$ & $0 \cdot 193$ & $49 \cdot 71$ & $0 \cdot 7968$ & 0.960 & $54 \cdot 18$ \\
\hline 0.7945 & $0 \cdot 225$ & $49 \cdot 9$ & $0 \cdot 7969$ & 0.992 or 1 & $54 \cdot 37$ \\
\hline 0.7946 & 0.257 & $50 \cdot 08$ & 0.7970 & $1 \cdot 031$ & 54.55 \\
\hline 0.7947 & $0 \cdot 289$ & $50 \cdot 26$ & 0.7971 & $1 \cdot 062$ & $54 \cdot 74$ \\
\hline 0.7948 & $0 \cdot 321$ & $50 \cdot 45$ & 0.7972 & $1 \cdot 093$ & $54 \cdot 92$ \\
\hline $0 \cdot 7949$ & $0 \cdot 353$ & $50 \cdot 68$ & 0.7973 & $1 \cdot 124$ & $55 \cdot 10$ \\
\hline 0.7950 & 0.385 & $50 \cdot 82$ & 0.7974 & $1 \cdot 155$ & $55 \cdot 29$ \\
\hline $0 \cdot 7951$ & 0.417 & $51 \cdot 01$ & $0 \cdot 7975$ & $1 \cdot 186$ & $55 \cdot 47$ \\
\hline $0 \cdot 7952$ & 0.449 & $51 \cdot 19$ & $0 \cdot 7976$ & $1 \cdot 217$ & $55 \cdot 65$ \\
\hline 0.7953 & $0 \cdot 481$ & $51 \cdot 38$ & 0.7977 & $1 \cdot 248$ & $55 \cdot 84$ \\
\hline 0.7954 & 0.512 & $51 \cdot 56$ & 0.7978 & $1 \cdot 279$ & $56 \cdot 02$ \\
\hline $0 \cdot 7955$ & 0.544 & $51 \cdot 75$ & $0 \cdot 7979$ & $1 \cdot 310$ & $56 \cdot 21$ \\
\hline 0.7956 & 0.576 & 51.94 & 0.7980 & $1 \cdot 341$ & $56 \cdot 39$ \\
\hline 0.7957 & 0.608 & $52 \cdot 12$ & 0.7981 & $1 \cdot 372$ & $56 \cdot 57$ \\
\hline 0.7958 & 0.640 & $52 \cdot 31$ & 0.7982 & $1 \cdot 403$ & $56 \cdot 76$ \\
\hline $0 \cdot 7959$ & $0 \cdot 672$ & $52 \cdot 49$ & 0.7983 & $1 \cdot 4.34$ & $56 \cdot 94$ \\
\hline $0 \cdot 7960$ & 0.704 & $52 \cdot 68$ & 0.7984 & 1.465 & $57 \cdot 13$ \\
\hline 0.7961 & 0.737 & $52 \cdot 87$ & 0.7985 & $1 \cdot 494$ or $1 \cdot 5$ & $57 \cdot 31$ \\
\hline
\end{tabular}




\section{MILK AND DAIRY PRODUCTS}

Table X.-For Estimating the Volatile Fatty Acids, according to Duclaux.

Mixture of Valeric and Acetic Acids :

Pure Valeric Acid

20 Valeric Acid : 1 Acetic Acid

10

$\begin{array}{rrr}10 & , \\ 8 & , & \\ 6 & , & \\ 5 & , & \\ 4 & , & \\ 3 & , & \\ 2 & , & \\ 1 & , & \\ 1 & , & \\ 1 & , & \\ 1 & , & \\ 1 & \text {, } & \\ 1 & \text { ", } & \\ 1 & \text { ", } & 10 \\ 1 & 20 \\ 1 & \text { Pure acetic acid }\end{array}$

\begin{tabular}{|c|c|c|c|c|c|c|}
\hline 20 & 30 & 40 & 50 & 60 & 70 & c.c \\
\hline $53 \cdot 0$ & 69.5 & $81 \cdot 0$ & 88.5 & $93 \cdot 5$ & $96 \cdot 5$ & \\
\hline $51 \cdot 2$ & $67 \cdot 3$ & $78 \cdot 6$ & $86 \cdot 2$ & $91 \cdot 4$ & $94 \cdot 8$ & \\
\hline $49 \cdot 5$ & $65 \cdot 3$ & 76.5 & $84 \cdot 2$ & $89 \cdot 6$ & $93 \cdot 3$ & \\
\hline $48 \cdot 8$ & $64 \cdot 4$ & $74 \cdot 4$ & $83 \cdot 2$ & $88 \cdot 7$ & $92 \cdot 5$ & \\
\hline $47 \cdot 6$ & 62.9 & $74 \cdot 0$ & $81 \cdot 7$ & $87 \cdot 3$ & $91 \cdot 4$ & \\
\hline 7 & $61 \cdot 8$ & $72 \cdot 8$ & $80 \cdot 6$ & $86 \cdot 1$ & $90 \cdot 6$ & \\
\hline$\cdot 4$ & $60 \cdot 2$ & $71 \cdot 2$ & $79 \cdot 0$ & $84 \cdot 9$ & $89 \cdot 4$ & \\
\hline 43.5 & $58 \cdot 0$ & 68.8 & $76 \cdot 6$ & $82 \cdot 8$ & $87 \cdot 6$ & \\
\hline $40 \cdot 4$ & $54 \cdot 1$ & $64 \cdot 7$ & $70^{\circ} 1$ & $79 \cdot 2$ & $84 \cdot 6$ & \\
\hline 1 & $46 \cdot 4$ & 56.5 & $64 \cdot 7$ & $77 \cdot 0$ & $78 \cdot 7$ & \\
\hline 8 & $38 \cdot 8$ & $48 \cdot 3$ & $56 \cdot 8$ & $64 \cdot 8$ & $72 \cdot 8$ & \\
\hline$\cdot 6$ & $34 \cdot 4$ & $44 \cdot 4$ & $52 \cdot 8$ & $61 \cdot 2$ & $69 \cdot 8$ & \\
\hline$\cdot 8$ & $32 \cdot 6$ & $41 \cdot 8$ & $50 \cdot 4$ & $59 \cdot 1$ & $67 \cdot 8$ & \\
\hline 5 & $31 \cdot 1$ & $40 \cdot 1$ & $48 \cdot 8$ & $57 \cdot 7$ & $66 \cdot 8$ & \\
\hline $20 \cdot 6$ & 30.0 & $39 \cdot 0$ & $47 \cdot 7$ & $56 \cdot 6$ & 66.0 & \\
\hline$\cdot 4$ & $28 \cdot 5$ & $37 \cdot 4$ & $46 \cdot 2$ & $55 \cdot 3$ & $64 \cdot 8$ & \\
\hline & $27 \cdot 6$ & $36 \cdot 4$ & $45 \div 2$ & $54 \cdot 4$ & $64 \cdot 1$ & \\
\hline & $25 \cdot 1$ & $34 \cdot 3$ & $43 \cdot 1$ & $52 \cdot 5$ & 63.1 & \\
\hline & $23 \cdot 4$ & $32 \cdot 0$ & $40 \cdot 9$ & $50 \cdot 5$ & $60 \cdot 9$ & \\
\hline
\end{tabular}

Mixture of Butyric and Acetic Acids:

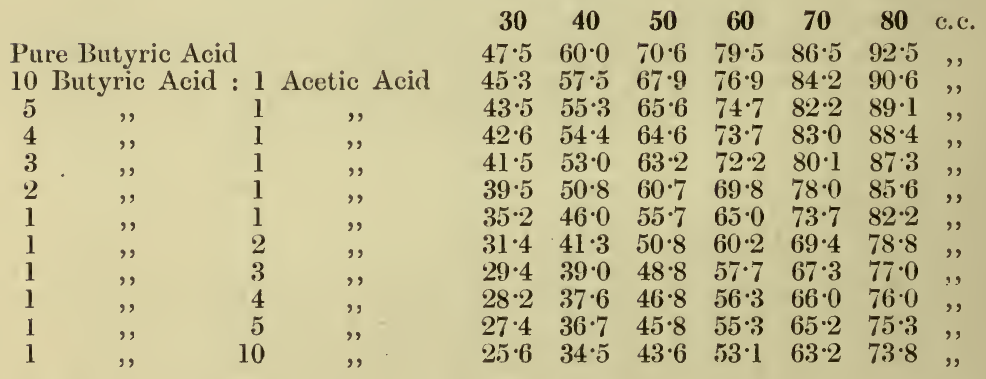

Mixture of Propionic and Acetic Acids :

\begin{tabular}{|c|c|c|c|}
\hline \multicolumn{4}{|c|}{ Pure Propionic Acid } \\
\hline 4 & ", & 1 & \\
\hline 3 & ", & l & , \\
\hline 2 & $"$, & 1 & , \\
\hline 1 & ", & l & ", \\
\hline 1 & ," & 2 & " \\
\hline 1 & ", & 3 & , \\
\hline 1 & , & $\begin{array}{l}4 \\
5\end{array}$ & $"$ \\
\hline
\end{tabular}

$\begin{array}{ccccccc}\mathbf{3 0} & \mathbf{4 0} & \mathbf{5 0} & \mathbf{6 0} & \mathbf{7 0} & \mathbf{8 0} & \text { c.c. } \\ 35 \cdot 3 & 46 \cdot 2 & 56 \cdot 8 & \mathbf{6 6} \cdot 7 & 76 \cdot 2 & 85 \cdot 0 & \text { ", } \\ 33 \cdot 3 & 43 \cdot 8 & 54 \cdot 2 & \mathbf{6 4} \cdot 0 & 73 \cdot 6 & 82 \cdot 8 & \text { ", } \\ 32 \cdot 9 & 43 \cdot 3 & 53 \cdot 6 & 63 \cdot 4 & 73 \cdot 0 & 82 \cdot 2 & \text { ", } \\ 32 \cdot 3 & 42 \cdot 6 & 52 \cdot 8 & 62 \cdot 6 & 72 \cdot 4 & 81 \cdot 7 & \text { ", } \\ 31 \cdot 3 & 41 \cdot 1 & 51 \cdot 9 & 61 \cdot 3 & 71 \cdot 1 & 80 \cdot 6 & \text { ", } \\ 29 \cdot 3 & 39 \cdot 1 & 48 \cdot 9 & 58 \cdot 6 & 68 \cdot 5 & 78 \cdot 5 & \text { ", } \\ 27 \cdot 4 & 36 \cdot 7 & 46 \cdot 2 & 55 \cdot 9 & 66 \cdot 0 & 76 \cdot 3 & \text { ", } \\ 26 \cdot 4 & 35 \cdot 5 & 44 \cdot 9 & 54 \cdot 5 & 64 \cdot 7 & 75 \cdot 2 & \text { ", } \\ 25 \cdot 8 & 34 \cdot 8 & 44 \cdot 1 & 53 \cdot 7 & 64 \cdot 0 & 74 \cdot 5 & \text { ", } \\ 25 \cdot 4 & 34 \cdot 4 & 43 \cdot 6 & 53 \cdot 2 & 63 \cdot 5 & 74 \cdot 0 & \text { ", }\end{array}$


Table X.-Continuerl.

Mixture of Valeric and Acetic Acids :

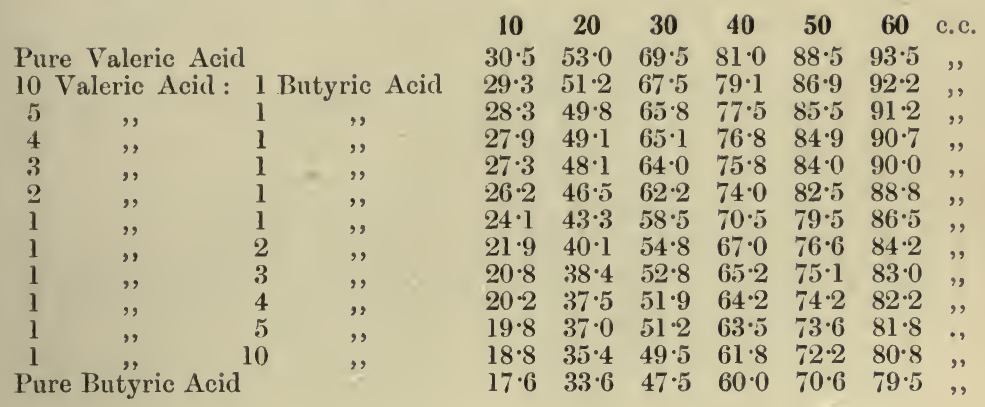

Mixture of Valeric and Propionic Acids :

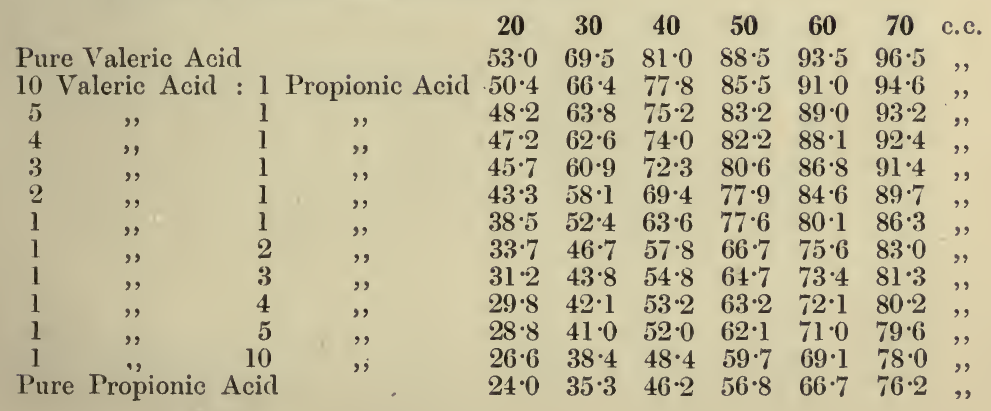

Mixture of Butyric and Propionic Acids:

\begin{tabular}{|c|c|c|c|c|c|c|c|c|}
\hline \multirow{3}{*}{$\begin{array}{l}\text { Pure Butyric Acid } \\
8 \text { Butyric Acid : }\end{array}$} & & 20 & 30 & 40 & 50 & 60 & 70 & c.c. \\
\hline & & $33 \cdot 6$ & $47 \cdot 5$ & $60 \cdot 0$ & $70 \cdot 6$ & $79 \cdot 5$ & 86.5 & \\
\hline & Propionic Acid & $32 \cdot 5$ & $46 \cdot 1$ & $58 \cdot 5$ & $69 \cdot 1$ & $78 \cdot 1$ & $85 \cdot 3$ & \\
\hline ," & ", & $31 \cdot 7$ & $45 \cdot 0$ & $57 \cdot 2$ & $67 \cdot 8$ & 770 & $84 \cdot 4$ & \\
\hline ," & ," & $31 \cdot 2$ & $44 \cdot 4$ & $56 \cdot 5$ & $67 \cdot 1$ & $76 \cdot 3$ & $83 \cdot 9$ & \\
\hline ", & ", & $30 \cdot 4$ & $43 \cdot 4$ & 5 & $66 \cdot 0$ & $75 \cdot 1$ & $83 \cdot 3$ & \\
\hline ", & ," & $28 \cdot 8$ & 41 & 5 & $63 \cdot 7$ & $73 \cdot 1$ & $1 \cdot 3$ & \\
\hline ", & ," & $27 \cdot 2$ & $39 \cdot 4$ & $50 \cdot 8$ & $61 \cdot 2$ & $71 \cdot 0$ & 79 & \\
\hline ," & ," & $26 \cdot 6$ & $38 \cdot 3$ & $49 \cdot 6$ & $60 \cdot 2$ & 6 & $78 \cdot 8$ & \\
\hline ," & & $25 \cdot 9$ & $37 \cdot 7$ & $49 \cdot 0$ & $59 \cdot 5$ & $69 \cdot 2$ & $78 \cdot 2$ & \\
\hline ," & ," & $25 \cdot 0$ & $36 \cdot 7$ & $47 \cdot 7$ & $58 \cdot 3$ & $68 \cdot 1$ & $77 \cdot 3$ & \\
\hline Pure Propionic Acic & & $24 \cdot 0$ & $35 \cdot 3$ & $46 \cdot 2$ & $56 \cdot 8$ & $66 \cdot 7$ & $76 \cdot 2$ & \\
\hline
\end{tabular}




\section{INDEX}

A

Abbe's refractometer, 148

Absorption number, iodine, 153

Acetone, 5

Acid, arachidic, 157

benzoic, detection of, 121

boracic, detection of, 120

butyric, 157, 183

capric, 157

caproic, 157,183

caprylic, 157

citric, 11

estimation of, 91

lauric, 157

myristic, 157

number of cheese fat, 234

oleic, 157

palmitic, 157

propionic, 183

salicylic, 183

stearic, 157

sulphuric, 71

Acidity of butter, 156

of cheese, 233

of milk, 2

determination of, 30

by Dornic's method, 32

Soxhlet's, 31

Thörner's, 32

Acids, coagulation of milk by, 180

fatty, insoluble, 153

soluble, 148

Ackermann's calculator, 104

Adams's paper coil method, 48

Added water in milk, determination of, 113

Addition of foreign fats to butter, 157

of preservatives to butter, 176

to milk, 119

of saccharate of lime to milk, 117

of water to butter, 175

to milk, 101, 112

Admixture of milk with goats' milk, 115

Adulteration of butter, detection of, 157

of cheese, 191

of milk, proofs of, 100

Aerobic bacteria, $\mathbf{2 1 6}, 217$

Aeroyenes, bacteria, 216
Albumin (lacto-albumin), 7

composition of, 8

estimation of, in milk, 85

in colostrum, 15

properties of, 8

Albumose and peptone nitrogen in cheese, 183,190

Alcohol, critical temperature of solution of milk fat in, 144

determination of, 226

test for acidity of milk, 30

Aldehyde-1'eductase in milk, 12

Alihin-Soxhlet method for estimation of milk sugar, 85

Amide nitrogen in cheese, 120

Aminoniacal nitrogen in cheese, 190

Amphoteric reaction of milk, 2

Amyl alcohol, 71

Amylene, 73

Anaerobic bacteria, 217

Analysis of butter, 135

of cheese, 185

of cream, 74

of milk, 30 et seq.

Annatto, 176, 178

Aniline yellow, 177, 179

Appeal to the cow, see Stall test

Arachidic acid, 157

Arachin, 5

Aræometer method of fat determination, 36

Arnold's guaiacum test, 97

Artificial colouring matters, detection of, 116

Ass, milk of, 16

Ash of milk, composition of, 10

determination of, 90

Aspergillus, 217

Automatic burette, 31

measures, 70, 71, 73

Average amount of fat in milk, 6 composition of milk, 16

\section{$\mathrm{B}$}

Babcock method for determination of fat, 61

Baby butyrometer, 65

Bucillus bulgaricus, 207

cancasicus, 206

facalis alcaligenes, 217 
Bacillus fluorescens liquefuciens, 217, 229

putrificus, 217

Bucterium lactis acidi, 218, 219 prodigiosum, 229

Baudouin's test for sesamé oil, 167

Beestings, 14

Benzoic acid in milk, detection of, 121

Bernstein's method for fat in butter, 139

Bicarbonate of soda, detection in milk, 119

Bichromate of potash as milk preservative, 20

estimation of, 90

Bixa orellana, 176

Boracic acid in milk, detection of, 120

Borax in milk, detection of, 120

Boiled milk, detection of, 97

Boiling test for acidity in milk, 30

Brine in butter, 131

Buddisel milk, 199, 212

Buffalo milk, composition of, 16

Butter, 130

adulterated with cocoanut oil, 168 margarine, 155

analysis of, 133,134

chemical composition of, 133, 134

colourers, 177, 178

physical properties of, 130

salt in, 130

sampling of, 134

specific gravity of, 131

thick, 132

fat, critical temperature of solution in alcohol, 144

density of, 142

examination of, 142

melting point of, 142

point of solidification, 143

properties of, 130

physical test of, 142

refractive index of, 145

solubility in alcohol, 144

solidification of, 143

specific gravity, 142

Buttermilk, 130

Butter yellow, 177

Butyric acid, 183

fermentation, 211

Butyro-refractometer, 145

\section{C}

Calcium, estimation of, 118

Calculation of abstracted cream, 114 adderl water, 113

Calves' stomach, 191

Cane sugar, letection of, in milk, 117 condensed milk, 213
Carbonate of soda in milk, 120

Carrots as butter colour, 178

Casein, action of rennet on, 8

analysis of, 215

composition of, 7

in milk, 7

estimation of, 84

lime compound, 7

preparations, 208

properties of, 7

separation of, 8

Catalase in milk, 12,98

Centrifuges, 72

Changes in milk due to sterilisation, 196

Cheese, adulteration of, 191

analysis of, 184

chemical composition of, 182,184

estimation of fat in, 186 et seq.

protein in, 190

water in, 185

water-soluble nitrogen compounds, 190

preparation of fat of, for analysis, 191

making of, 180

rennet, 180

ripening of, 181

sampling of, 184

sour milk, 182

Chemical composition of butter, 133 cheese, 182

milk, 16

examination of milk, 30

Cholesterine, 12, 163

Churning of cream, 130

Citric acid in milk, 11 estimation of, 91

Cladosporium butyri, 229

Clear butter, 132

Coagulation of milk by rennet, 8

Cocoanut oil, 168 detection of, in butter, 173

Coli bacteria, 216

Colostrum, 14 chemical composition of, 15 microscopic appearance of, 15

Colour of milk, 1

Colouring matter in butter, 176

Condensed milk, 200 analysis of, 213 separated milk, 202

Condition of fat in milk, 5

Conditions of action of rennet, 194

Constituents of ash of milk, 10

Copper solution, reduction of, by milk sugar, 10

Corps granuleux, 15

Correction of specific gravity for changes of temperature, 236

Cow, composition of milk of, 16 
Cream, analysis of, 75 artificial thickening of, 117

Creatinin in milk, 12

Critical temperature of solution of butter fat in alcohol, 144

Crismer number, 144, 161

Crismer's method, 144

Cupric oxide method for estimation of milk sugar, 87

Curcuma, 177, 178

Curd, acid, 180 rennet, 180,182

Curdling due to lactic acid, 180

\section{D}

Dark field photographs of butter, 132 , 133

Decomposition products of butter, 229

of cheese, 233

of milk, 216

Degree of acidity of butter, 144

of cheese, 233

of oxidation of butter, 232

of rancidity of butter, 230

Detection of added water in butter, 176

in milk, 101

adulteration in butter, 157

in cheese, 191

artificial colouring in milk, 116

benzoic acid, 121

boracic acid, 120

cane sugar in milk, 117

colouring matter in butter, 176

in milk, 116

formaldehyde, 121

goats' milk in milk, 115

hydrogen peroxide, 122

lime in milk, 118

nitrates in milk, 101

saccharose in milk, 117

separated milk in milk, 112

Determination of acidity of butter, 156

of milk, 30

anhydrous solids, not fat, in butter, 140

common salt in butter, 141

citric acid in milk, 91

fat in butter, 137

in cheese, 186

in cream, 56,75

in milk, 35

by Adams' method, 48

Babcock's method, 61

Gerber's method, 69

Gerber's "sal" method, 81

Lactocrite method, 58
Determination of acidity by Liebermann-Székely's method, 55

Lindström's method, 65

Nilson's kaolin method, 58

Röse-Gottlieb's method, 52

Sichler's method, 77

freezing point of milk, 106

Hehner number, 153

iodine number, 153

Koettstorfer number, 152

nitrogenous substances in milk, 80

potassium bichromate in milk, 90

protein in butter, 141

in cheese, 190

in milk, $80,83,84$

refractive index of butter fat, 145 of milk serum, 108

Reichert-Mcissl number, 148

specific gravity of milk, 23

of milk serum, 105

water in butter, 135 in cheese, 185

Dicalcium casein, 7

Dinitronaphthol, 177

Dispora caucasica, 206

Dirt, estimation of, in mlk, 26 of milk, 13

Dried milk, 202, 214

Dry matter of milk, 3

determination of, 33

Drying oven, Soxhlet's, 34

Duclaux's method for estimation of volatile fatty aids, 221

\section{E}

Earth nut oil, 167

Enzymes in cheese, 181

in milk, 12

in ripening of cheese, 181

Ester number, 231

Estimation of acidity, 30

albumin in milk, 85

albuminose in cheese, 234

alcohol, 226

amide nitrogen in cheese, 234

aumoniacal nitrogen in cheese, 234

ash of milk, 90

calcium, 118

casein in milk, 84

citric acid in milk, 91

common salt in butter, 141

curd in butter, 141

dirt in milk, 26

fat in butter, 137

in cheese, 186

in cream, 56

in milk, 35 et seq.

hydrogen peroxide, 123

insoluble fatty acids in butter, 153 , 169 
Estimation of iodine number, 153

lactic acid, :2ls

lecithin in milk, 94

milk sugar, 8.5

nitrogen in butter, $1+1$

in cheese, 190

refractive index of butter, 145, 159 of milk fat, 40

of milk serum, 108

solids, not fat, in butter, $1+0$

soluble volatile fatty acids, 148

total nitrogen in milk, 80 protein in milk, 83, 84

water in butter, 13.5

in cheese, 185

Eucasein, 209

Eulactol, 209

Ewe, milk of, 16

Examination of butter, 134 of milk, 17

Extraction methorls for the determination of fat, 47

\section{$\mathrm{F}$}

Factors influencing fat content of milk, 6

Fat, amount of, in milk, 6 composition of, in milk, 5 determination of, in butter, 137

in condensed milk, 213

in cheese, 186

in cream, 51,56

in milk, 35

in separated milk, 51

of nilk, $\mathbf{j}$

melting point of, 5

refractive index of, 5

size of globules, 4

Fatty acids in butter, 157

Fehling's solution, 86;

Fermentation, alcoholic, $205,2.26$

butyric, 131, 229

lactic acid, 9, 183, 211

test, 124

Fleischmann's formula, 103

Flocculent matter in milk, 2:2

Formaldehyde as preservative,, 20 detection of, 121

Formalin, see Formaldehycle

Formin, 5

Formula for calculating total solids in milk, 103

Weibull's sour milk method, 26

Free acid in butter, 156

Freezing point of milk, 2, 106

Fritzmann's nitrate test, 10.2

(:

Galactase in milk, 12, 98

(rases in milk, 12
Gerber's method, 69, 189

"sal " method, so

Globules of fat in milk, $t$

Globulin, 7

Glycerides in butter, 1.57

in milk fat, $\tilde{5}$

Goats' milk, addition of, 11 j

cheese, 181

Granulobacilli, 217

Gravimetric method for estimation of milk sugar, 85

$\mathrm{H}$

Heat, action of, on milk, 96, $19 \mathrm{~s}$

Heated milk, tests for, $9 \bar{\tau}$ et seq.

Heimer number, 153

Homogenised milk, 198

Human milk, composition of, 16

Hydrogen peroxide, detection of, 1222 estimation of, 123

Hypoxanthine in milk, 12

I

Insoluble fatty acids, 153

Iodine-mercuric chloride solution, 154

Iodine number, 153

Hübl's method, 154

Wijs' method, 155

J

Jensen's nethod for estination of volatile fatty acids, $2 \cdot 20, \cdot 2 \cdot 21$

\section{K}

Kaolin method for determination of fat, 50

Kephir, 9, 205

composition of, 206

fermentation, 206

grains, 20.5

bacteria in, 20.5

Koettstorfer number, 15:, 162

Kolibri butyrometer, 67

Koumiss, 9, 205

composition of, 207

L

Lactic acid, 9

bacteria, 9

estimation of, 218 
Lactic acid fermentation, 9, 183, 211

Lactoalbumin, 7

composition of, 8

specific rotation of, 9

Lactocaramel, 9

Lactocrite method for determination of fat, 58

Lactoglobulin, 79

Lactometer, 23

Quevenne's, 25

Soxhlet's, 24

Lactose (see Milk sugar), 9

Latent colour of margarine, 166

Lauric acid, 157

Laurin, 5

Leben, 207

Lecithin in milk, 11 estimation of, 94

Leffmann-Beam modification, 150

Leucocytes, 13

Leucine, 12, 183

Liebermann-Székely's method, 55

Lime-casein compounds, 7

Lime, determination of, in milk, 118

Lindström's butyrometer method, 65

Lysine, 183

M

Mammals, milk of, 16

Mare, milk of, 16

Mare's milk Koumiss, 9

Margarine in butter, 158

detection of, 159 et seq.

preparation of, 158

properties of, 158,175

Marigolds, 177

Martius yellow, $17 \%$

Mazoun, $20 \%$

Measurer for amyl alcohol, 70, 73

cream, 75

mercury, 81

sulphuric acid, 70,71

Melting point of butter fat, 142

Membrane round fat globules, 5

Micro-organisms in milk, 13

Microphotograph of colostrum, 16

fat globiles, 4

separator slime, 14

Milk, acidity of, 2,30

adulteration, proofs of, 100

amphoteric reaction of, 2

appearance of, I

boiling point of, 2

Buddised, 212

composition of, 3

condensed, 213

decomposition products of, 216

desiccated, 214
Milk, dry matter of, 4

electrical resistance of, 2

fat globules in, 4

freezing point of, 2

heating of, 2

of mammals, 16

powder, 214

preserved, 196

products of decomposition of, 216

refractive index of, 2

specific gravity of, 1

specific heat of, '2

sterilised, 196, 210

riscosity of, 1

yield of cows, 6

Milk fat, 5

composition of, 5

condition of, in milk, 5

melting point of, 5

point of solidification of, 5

refractive index of, 5

specific gravity of, 5

Milk powiler, 202 analysis of, 214

Milk salts, composition of, 11 serum specific gravity of, 105 refractive index of, 108

Milk sugar, 9 action of bacteria on, 9 yeasts on, 9 chemical properties of, 9 estimation of, $85,87,89$ fermentation of, 9

reducing properties of, 10 solubility of, 9

Milk tabloids, 204

Mixing of water with butter, 165

Mucor, 217

Myristic acid, $15 \%$

Myristin, j

\section{$\mathrm{N}$}

New butter number (Polenske's), 169

Nilson's kaolin method, 50

Nitrate test for water in milk, 101, 102

Nitrogen in milk, determination of total, 80

amide in cheese, 190, 234

total in cheese, 190

Nuclein, 12

Nutrose, 209

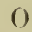

Oidlium lacti:, 217, 2.25)

Oil, cocoanut, 168

sesamé, $16^{7}$ 
Oleic acid, 15,

Olein, 5

Oxidation of butter, 230

Oxpropionic acid, set Iactic acid

\section{'}

Palmitic acisl, 15;

Palmitin, J

Paracasein, 8, 186,

Paraplienylenediamine for detection of heated nilk. 98

Pasteurisation, proof of. 99, 141

Pasteurised milk. 198

Payment for milk, 35

Penicillium, 217, 2.29

Peptone in inilk, 12 fermentation, 211

Peptonising bacteria, 22

Peroxidase in milk, 12, 98

Phosphates in ash of milk, 11

Phosphorus in casein, 10

Physical examination of butter milk, $2 \%$

Phytosterine, 163 acetate test, 16 ?

Plasmon, 209

Point of solidification of butter fat, 143

Polarimetric methol for estimation of milk sugar, 8 ;

Polenske's new butter number, 16!)

Potassium bichromate, 20 test for, 90

Preservatives for milk, 21)

in butter, 176

in milk, 119

Preserved milk, 196

Products of ripening of cheese, 183

Proof of Pasteurisation, 141

Propionic acid, 8:3

Protein, determination of, in lutter, 141

in cheese, 190

in milk, $80,8: 3,84$

colostrum, 1.j

milk, composition of, $\overline{7}$

Protoll, 209

Pyknometer, 25

F

Rancid butter, 131

Raw and heated milk, to distinguish, 96

Reaction of milk, 30

Reddish tinge in milk, ․․ㄹ

Reductase test, 128

Reduction by milk sugar, $10,8 \bar{i}, 87$
Refractive index of butter, 145, 159 milk serum, 108

Refractometer, Abbé, 148 immersion, 110

Wollny-Zeiss, 40, 145

methorl for rletermining fat in milk, 40

milk sugar, 89

water in milk, 108

Reicliert-Meissl number, 148, 161

Reincleer, milk of. 16

Remoral of creaun from milk, 111, 112

Renuet, 191

action on casein, 8, 180

conditions influencing action of, 4.14

cheese, $18: 2$

preparation of, 191

powder, 192

testing of, 191

Riegel's milk protein, 209

Ripening of cream, 130 of cheese, 181, 183 products of, 182,233

Rise of specific gravity of milk on standing, 23

Rising of cream, 5

Rittliasen's methor for estimation of protein, 83

Röse-Crottlieb's methor, 52, 138, 187

Rouser for mixing milk, 17

\section{S}

Sacchaiomycex kifir, 203

Saccharate of lime in milk, 117

Saccharose, detection of, 117

satflower yellow, 179

Sattlowers, $17 \%$

Saffion, $17 \%, 179$

Salt. estimation of, in butter, 141 in butter, 130

Salts of milk, 11

Salicrlic acid, detection of, in milk, 120

Sampling of butter, 134 of cheese, 184

of milk, 17

Sanatogen, 2(1)

Sichardinger's reageut, 211

Schiff's reagent, 122,232

Schmid-Bondzynski method, 18-

Sebelien's method for total protein, 84

Sediment,

Separated condensed milk, 20:2 milk, detection of, in inilk, 112

Separator slime, 13

Serum, determination of specific gravity of, 105 
Sesamé oil, 167 test for, 167

Sichler's sinacid method, 7

Size of globules, 4

Skim milk, see Separated milk cheese, 181

Smell of milk, 1, 23

Sodium thiosulphate solution, 154

Soft cheese, 181

Solid fatty acids, 157

Solicls not fat, estimation of, in butter, 140

total, in milk, 33

Soltsein's test for sesamé oil, 168

Solubility of butter fat in alcohol, 144

milk sugar, 9

Soluble fatty acids, estimation of, 148,161

Solution, Fehling's, 86

Sour milk, analysis of, 21 cheese, 182

Soxhlet's drying oven, 34

extraction apparatus, 49

lactometer, 24

method of fat determination, 36 nitrate test, 101

Specific gravity of butter fat, $1+2$ milk, 1

determination of, 23

serum determination of, 10.5

Stall test, 112

Standard for fat in milk, 3.)

Stearic acid, $15 \%$

Stearin, 5

Sterilisation, fractional, 197

Sterilised milk, 196, 210

detection of, in milk, 99, 210)

Storch's test for heated milk, 98

Succinic acid, 183

Sulphur in easein, 10

Supply of water at constant temperature, 42

Sweetened condensed milk, 200

\section{$\mathrm{T}$}

Taste of milk, 1

Test, fermentation, 124 milkings, apparatus for, 19 stall, 112

Testing of rennet, 191

Tests for benzoic acid, 121

bicarbonate of soda, 119
Tests for boracic acid, 120

borax, 1:20

formaliehyde, 1:21

hydrogen peroxicle, 1.2.2

salicylic aciel, 123

"Thick" butter, 132

Torula liefir, 206

Total acid number, $2: 31$

nitrogen in cheese, 190

in milk, 80

solids of milk, 3 , 3.j

determination of, 33

Tricalcium casein, 7

Tyrosine, 183

U

Unsweetened condensed milk, 202

Urea in milk, 12

V

Variation in composition of milk, 2 percentage of fat in milk, 6

Victoria yellow, 177,179

Volatile acid number, 231

fatty acids, estimation of in butter, 219

in cheese, 233

IV

Water, estimation of, in butter, 136 in cheese, 18.5

Weibull's method for estimation of fat in cream, 56

Westphal balance, 25

Whale, milk of, 16

Whey albumin, 8,180

Wollny-Zeiss refractometer, 41

Wollny's refractometer method, 40

Woman's milk, 16

\section{$Y$}

Yeast, 20.5

Yields of milk, 6

Yorghut, 207

bacteria in, 207

composition of, 207 

THIS BOOK IS DUE ON THE IAST DATE STAMPED BELOW

AN INITIAL FINE OF 25 CENTS WILL BE ASSESSED FOR FAILURE TO RETURN THIS BOOK ON THE DATE DUE. THE PENALTY WILL INCREASE TO 50 CENTS ON THE FOURTH DAY AND TO \$1.00 ON THE SEVENTH DAY OVERDUE.

$\operatorname{Arr} 151962 / 6$

LIBRARY, COLLEGE OF AGRICULTURE, DAVIS UNIVERSITY OF OALIFORNIA

$5 m \cdot 4,41(2361 \mathrm{~s})$ 
